University of Louisville

ThinkIR: The University of Louisville's Institutional Repository

$12-2020$

\title{
Photoelectrical and photoelectrochemical characterization of the materials used in dye-sensitized and perovskite solar cells.
}

Pom Lal Kharel

University of Louisville

Follow this and additional works at: https://ir.library.louisville.edu/etd

Part of the Chemistry Commons

\section{Recommended Citation}

Kharel, Pom Lal, "Photoelectrical and photoelectrochemical characterization of the materials used in dyesensitized and perovskite solar cells." (2020). Electronic Theses and Dissertations. Paper 3534.

https://doi.org/10.18297/etd/3534

This Doctoral Dissertation is brought to you for free and open access by ThinkIR: The University of Louisville's Institutional Repository. It has been accepted for inclusion in Electronic Theses and Dissertations by an authorized administrator of ThinkIR: The University of Louisville's Institutional Repository. This title appears here courtesy of the author, who has retained all other copyrights. For more information, please contact thinkir@louisville.edu. 


\title{
PHOTOELECTRICAL AND PHOTOELECTROCHEMICAL CHARACTERIZATION OF THE MATERIALS USED IN DYE- SENSITIZED AND PEROVSKITE SOLAR CELLS
}

\author{
By \\ Pom Lal Kharel \\ A Dissertation \\ Submitted to the Faculty of the \\ College of Arts and Sciences of the University of Louisville \\ in Partial Fulfillment of the Requirements \\ for the Degree of \\ Doctor of Philosophy in Chemistry \\ Department of Chemistry \\ University of Louisville \\ Louisville, Kentucky
}

December 2020 
Copyright 2020 by Pom Lal Kharel

All rights reserved 

PHOTOELECTRICAL AND PHOTOELECTROCHEMICAL CHARACTERIZATION OF THE MATERIALS USED IN DYE-SENSITIZED AND PEROVSKITE SOLAR CELLS

\author{
By \\ Pom Lal Kharel
}

A Dissertation Approved on

July 30, 2020

By the following Dissertation Committee

\begin{tabular}{c} 
Dissertation Chair \\
Dr. Francis P. Zamborini \\
\hline Dissertation Co-Chair \\
Dr. Bruce P. Alphenaar \\
\hline Committee member \\
Dr. Thad Druffel \\
\hline Committee member \\
\hline Dr. Farshid Ramezanipour \\
\hline
\end{tabular}

Committee member

Dr. Xiang Zhang 


\section{DEDICATION}

***I dedicate my Ph.D. dissertation to my beloved spouse Kamala Ghimire, son Prikash Kharel, daughter Pranvee Kharel, and parents

Nandaram Kharel and Dilkumari Kharel.*** 


\section{ACKNOWLEDGEMENTS}

First of all, I would like to express my sincere gratitude to my advisor Dr. Francis P. Zamborini and co-advisor Dr. Bruce W. Alphenaar for their excellent guidance and support throughout my graduate studies. They always encouraged me to develop deep scientific knowledge in the field of photovoltaics. I am grateful to them for giving me an opportunity to work in their labs for my Ph.D. studies.

I am grateful to my dissertation committee members Dr. Farshid Ramezanipour, Dr. Xiang Zhang, and Dr. Thad Druffel for providing me with valuable suggestions to develop my research projects and also to improve my dissertation write up. My deep appreciation goes to my lab mate Dr. Kasun Fernando for his help and contribution towards the development and successful completion of my research projects. I am equally grateful to all other lab mates and former undergraduate students, who directly or indirectly supported me. I am indebted to Mr. Steve Riley who was always there to help me in fixing analytical instruments. I would like to thank the Department of Chemistry for supporting me through Graduate Teaching Assistantship and NSF funding to support my research financially. Lastly, I am grateful to my beloved spouse Kamala Ghimire, my kids Prikash Kharel and daughter Pranvee Kharel for their constant encouragement and support to pursue my $\mathrm{PhD}$ degree. 


\title{
ABSTRACT
}

\section{PHOTOELECTRICAL AND PHOTOELECTROCHEMICAL CHARACTERIZATION OF THE MATERIALS USED IN DYE- SENSITIZED AND PEROVSKITE SOLAR CELLS}

\author{
Pom Lal Kharel
}

July 30, 2020

Solar energy is one of the most important alternative renewable energy sources to fulfill the increasing demand of energy in the world. Third-generation solar cells like dyesensitized solar cells, perovskite solar cells, quantum dot solar cells, and organic solar cells are extensively studied to increase their photoconversion efficiency, and ultimately for their large-scale implementation. A dye-sensitized solar cell consists of a photoanode of a mesoporous film of titania sensitized with dye sandwiched with a counter electrode, which is usually a platinum-coated transparent conducting oxide, and a redox couple injected between the photoanode and counter electrode. Doping titania with rare-earth metal oxides (REOs) has been an interesting approach to improve the conversion efficiency of dyesensitized solar cells. REOs have been doped into titania paste to show an improvement in the photovoltaic performance of dye-sensitized solar cells, however, most of the reported cells are not efficient enough to conclude whether the enhancement is due to doping or it is because of the cell quality. We incorporated nanoparticles (NPs) of REOs in titania paste and built highly reproducible dye-sensitized solar cells using amphiphilic C101 dye and iodide/triiodide redox couple in nitrile-based solvent (Z960 electrolyte). The doping level for optimized cells was $2.0 \%$ for neodymium oxide and $1.0 \%$ for erbium oxide. We did 
the measurements of photocurrent, impedance, incident photon-to-electron conversion efficiency (IPCE), and dye loading to investigate the mechanism of enhancement of the photovoltaic performance by REO NPs. Electrochemical impedance spectroscopy measurements showed that doping with REO decreased the total impedance of the cell and IPCE measurements revealed enhanced photon absorption by the dye in REO-doped cells. In the same fashion, the REO-doped anodes showed larger dye loading compared to undoped anodes, which was maximum for $1.0 \%$ doping of erbium oxide and $2.0 \%$ doping of neodymium oxide. REOs not only enhance dye adsorption but also facilitate electron transport through the mesoporous layer, thereby increasing the collection efficiency of the photoexcited electrons.

To further explore the mechanism for the interaction between REO NPs and titania, an electrical and electrochemical study of REO-doped nanostructured titania films was performed. Doped films were found to be 40-50 times more conductive than undoped films, with linear current-voltage characteristics. Cyclic voltammograms of doped samples showed an enhanced scan rate dependence in the deep trap regime due to a slower charge trapping rate. Finally, electrochemical impedance measurements revealed a decrease in space charge density and a shift in the flat-band potential. Taken together, these results suggest that charge transfer from the REO neutralizes the deep trap states in the nanostructured titanium dioxide (NTD) film, decreasing charge scattering, and improving the NTD performance as an electron acceptor and electron transport material.

Perovskite solar cells (PSCs) were first made when the dye-loaded semiconductor of dye-sensitized solar cell was replaced by perovskite layer and liquid electrolyte by a hole transport layer. The light harvesting perovskite layer is sandwiched between electrontransport and hole transport layers. Organic-inorganic perovskites, also known as hybrid perovskites have fascinating optoelectronic properties for their applications in highly efficient solar cells. The stability in ambient conditions and hysteresis in current-potential curves are two main challenges. The ease with which the separation of photogenerated charge carriers, electron-hole pairs (excitons), takes place is very critical for the performance of PSCs. In addition to the work function difference of electron-transport and hole transport layers, the intrinsic built-in potential in the perovskite films can play a 
significant role in the separation of these excitons. The internal electric originates from the local polarization of the film due to non-centrosymmetric lattice and ionic polarization and can be measured through an $\mathrm{AC}$ photocurrent technique. The polarization of a pristine sample is strongly dependent on the size of grains and can be used to determine the quality of the film. After poling the film by applying a potential through interdigitated $\mathrm{Au}$ electrodes, the devices with different grain sizes behaved differently upon relaxation.

We observed that the polarization of a mixed halide hybrid perovskite film strongly depends on the background environment. The Quartz Crystal Microbalance measurements reveal that the perovskite film adsorbs Ar gas in the presence of solar light. The combination of Ar gas and solar illumination results in the enhancement of the electric polarization of the mixed halide hybrid perovskite film. Consequently, the photocurrent is increased due to the stronger driving force for the separation of excitons. This observation is illustrated in an actual PSC where the photovoltaic enhancement is observed with Ar gas. Our results suggest that the contribution from the background environment should be taken into consideration when describing the photovoltaic performance of a PSC. 


\section{TABLE OF CONTENTS}

ACKNOWLEDGEMENTS.............................................................................. iv

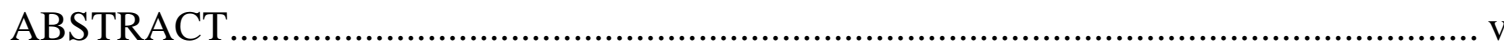

LIST OF TABLES ...................................................................................... xiii

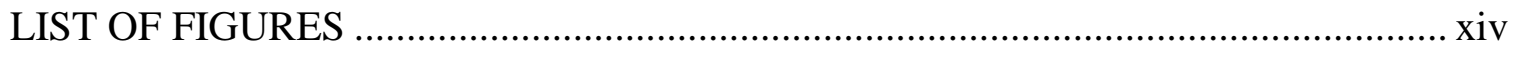

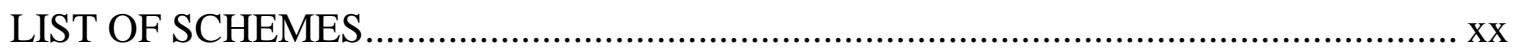

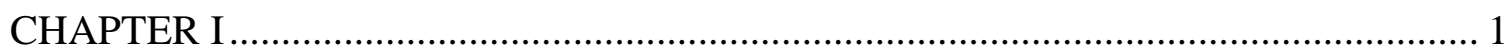

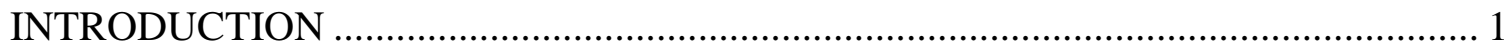

1.1. Demand of Renewable Sources of Energy ..................................................... 1

1.2. Solar Cells ................................................................................................... 2

1.2.1. Architecture and Working Principle of Solar Cells .......................................... 2

1.2.2. Photoelectrochemical Parameters of Solar Cells ........................................... 4

1.2.3. Semiconductors Used in Different Generation Solar Cells ............................... 5

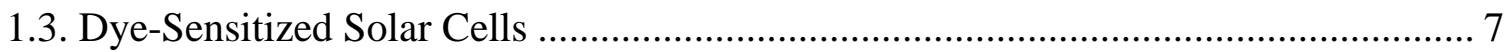

1.3.1. Components of Dye-Sensitized Solar Cells ................................................... 7

1.3.2. Working Principle of Dye-Sensitized Solar Cell ........................................ 11

1.3.3. Photovoltaic Performance of Dye-Sensitized Solar Cells and Challenges ......... 14

1.3.4. Motivation for the Study of Dye-Sensitized Solar Cells ................................. 15

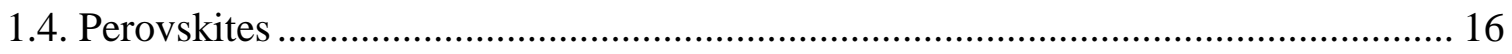

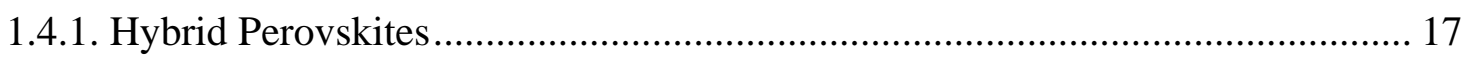

1.4.2. Electric Polarization in Hybrid Perovskites ................................................... 19

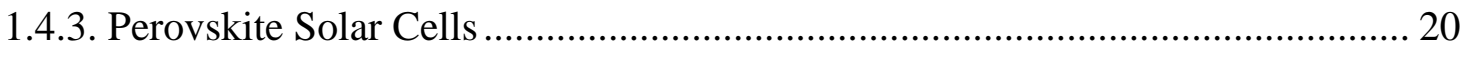

1.4.4. Working Principle of Perovskite Solar Cell.................................................. 20 
1.4.5. Issues with Perovskite Solar Cells ............................................................. 22

1.4.6. Two-Dimensional Hybrid Perovskites....................................................... 23

1.4.7. Motivation for the Study of Perovskites ..................................................... 24

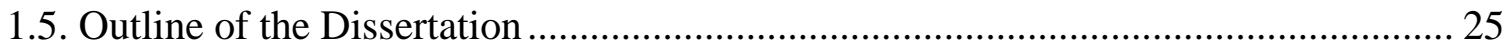

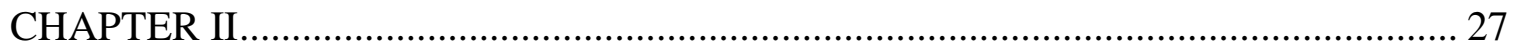

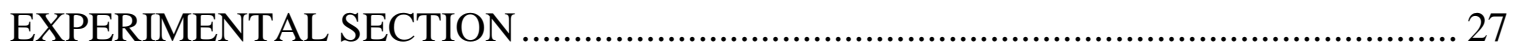

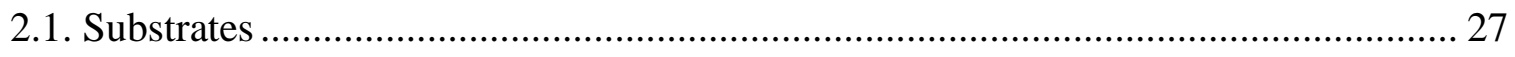

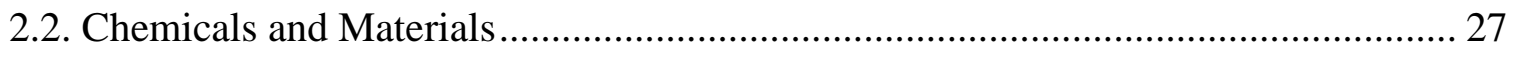

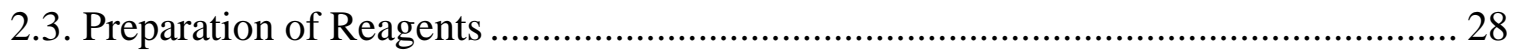

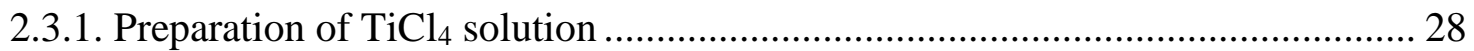

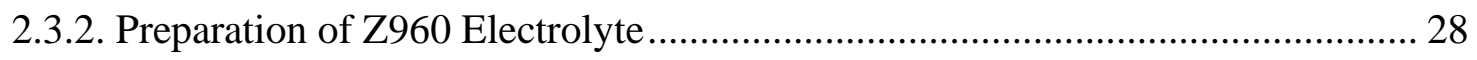

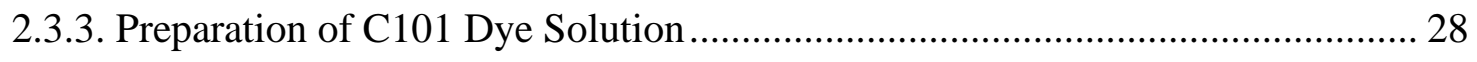

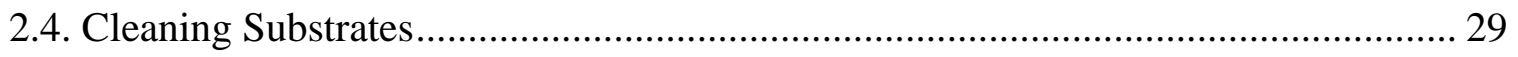

2.5. Fabrication of Dye-Sensitized Solar Cells ......................................................... 29

2.5.1. Preparation of Titania Paste for Dye-Sensitized Solar Cell ............................ 29

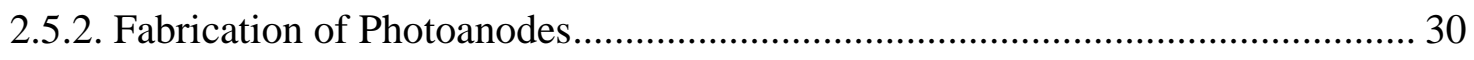

2.5.3. Sensitization of Photoanodes with Dye ...................................................... 33

2.5.4. Fabrication of Counter Electrodes ......................................................... 33

2.5.5. Fabrication of Sandwich Dye-Sensitized Solar Cell ........................................ 34

2.6. Characterization of Photoanodes Used for Dye-Sensitized Solar Cells ................... 37

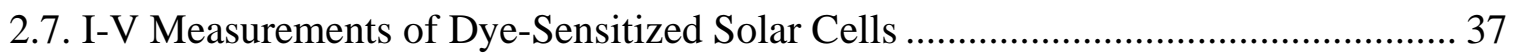

2.8. Electrochemical Impedance Spectroscopy (EIS) Measurements of Dye-Sensitized

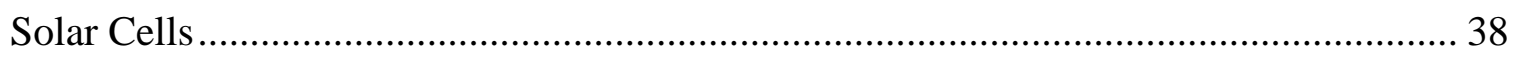

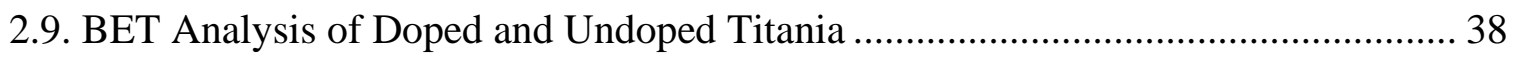

2.10. Dye Loading in Doped and Undoped Titania ................................................... 38

2.11. Synthesis of Methylammonium Chloride ........................................................ 38

2.12. Preparation of $\mathrm{CH}_{3} \mathrm{NH}_{3} \mathrm{PbCl}_{x} \mathrm{I}_{3-\mathrm{x}}$ Precursor Solution......................................... 40 
2.13. Fabrication of Highly Granular Film ...................................................................... 40

2.14. AC Photocurrent Measurement......................................................................... 41

2.15. Calculation of Average Grain Size .................................................................... 46

2.16. Quartz Crystal Microbalance (QCM) Measurements ................................................ 48

2.16. Fabrication of Perovskite Solar Cell ...................................................................... 49

2.17. Photoelectrochemical Measurements of Perovskite Solar Cells............................... 51

2.18. Synthesis of Crystals of Layered and 3D Hybrid Perovskites ................................ 53

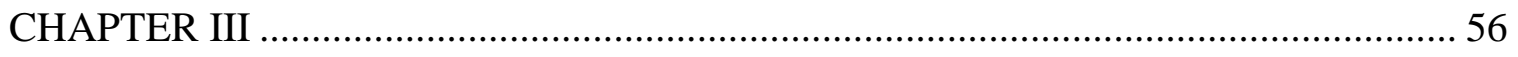

ENHANCING THE PHOTOVOLTAIC PERFORMANCE OF DYE-SENSITIZED SOLAR CELLS WITH RARE-EARTH METAL OXIDE NANOPARTICLES ............. 56

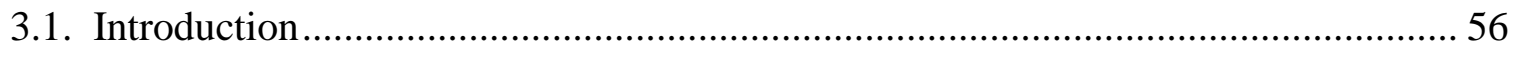

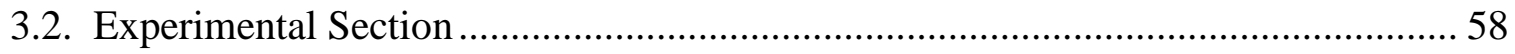

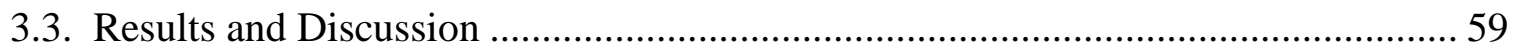

3.3.1. Current Density-Potential Characteristics....................................................... 59

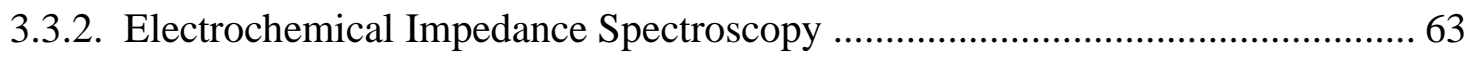

3.3.3. Optimization of Concentration of REO Nanoparticles and Dye Loading ......... 64

3.3.4. Morphology of Doped and Undoped Films ...................................................... 65

3.3.5. BET Analysis of Doped and Undoped Titania ................................................. 68

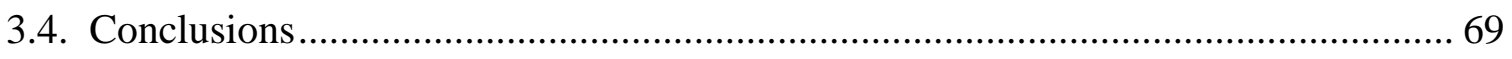

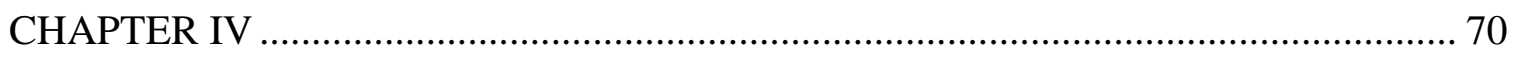

EFFECT OF RARE-EARTH METAL OXIDE NANOPARTICLES ON THE CONDUCTIVITY OF NANOCRYSTALLINE TITANIUM DIOXODE: AN ELECTRICAL AND ELECTROCHEMICAL APPROACH .......................................... 70

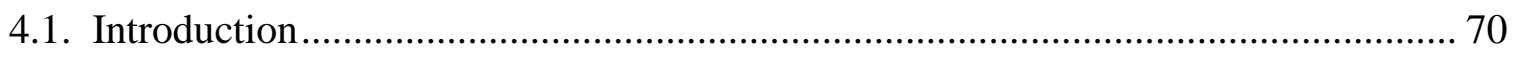

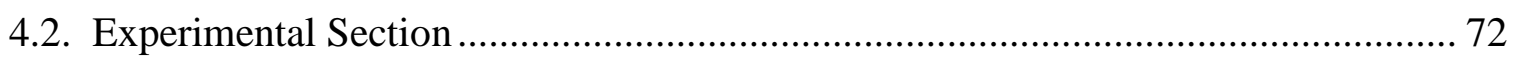

4.3. Results and Discussion ................................................................................... 74

4.3.1. Electrical Conductivity Measurements ............................................................. 74

4.3.2. Electrochemical Measurements ...................................................................... 77 
4.3.3. Model for Charge Trapping and Detrapping Kinetics .................................. 80

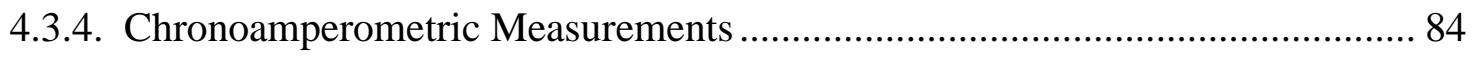

4.3.5. Electrochemical Impedance Measurements ............................................... 85

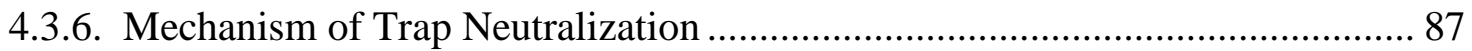

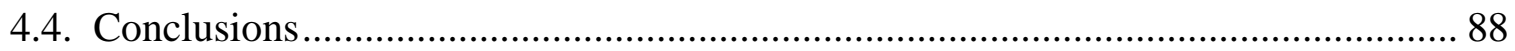

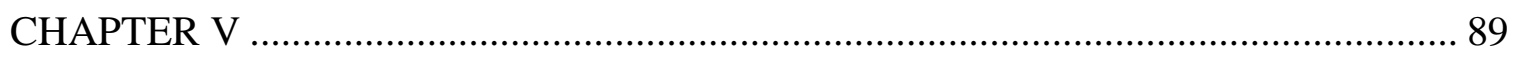

AN AC PHOTOCURRENT METHOD FOR CHARACTERIZATION OF THE

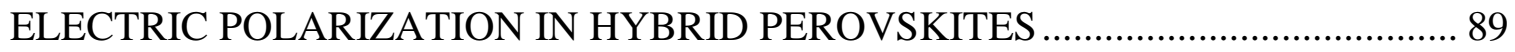

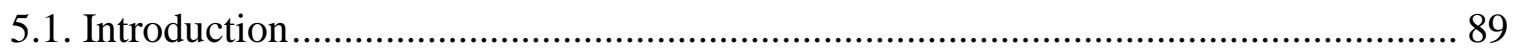

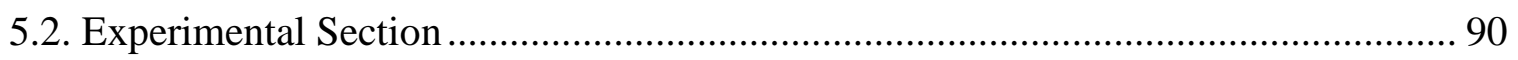

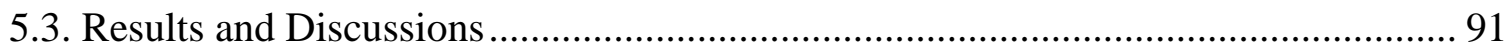

5.3.1. Highly Granular Film and its Characterization...................................... 91

5.3.2. Variation of AC Photocurrent with External Electric Field.............................. 92

5.3.3. Built-in Potential and Electric Polarization ................................................ 94

5.3.4. Grain Size Dependence of Intrinsic Polarization .......................................... 96

5.3.5. Carrier Relaxation after Poling ............................................................... 98

5.3.6. Polarization-Electric Field Loops in Perovskite Films ................................ 100

5.3.7. Origin of Intrinsic Polarization and Effect of Ionic Concentration ................ 101

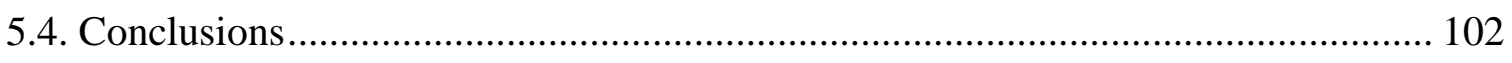

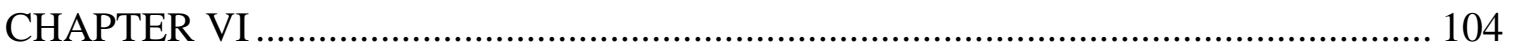

EFFECT OF LIGHT SOAKING IN ARGON ON MIXED HALIDE HYBRID

PEROVSKITE: ENHANCED ELECTRIC POLARIZATION AND IMPROVED

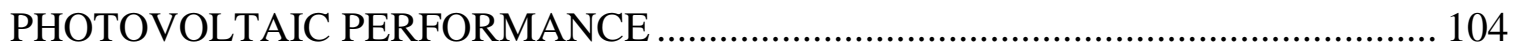

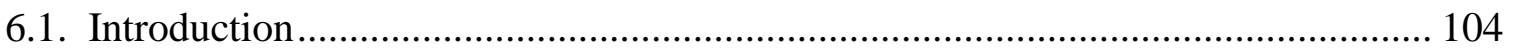

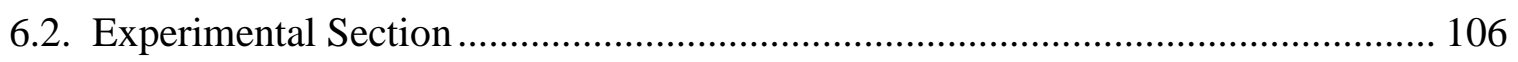

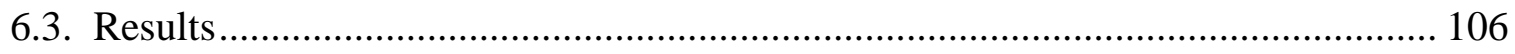

6.3.1. Electric Polarization in Ar with Light.................................................... 106

6.3.2. AC Photocurrent in Ar with Light ......................................................... 108 
6.3.3. Effect of Background Gases on the Photocurrent Spectrum ............................. 109

6.3.4. Quartz Crystal Microbalance (QCM) Measurements ....................................... 112

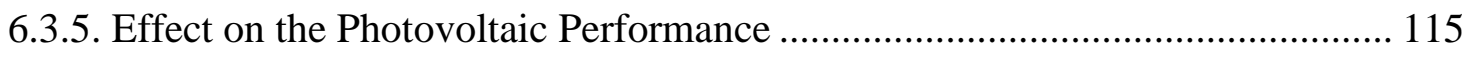

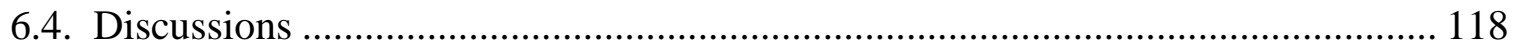

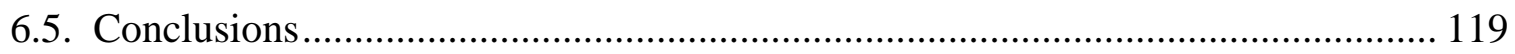

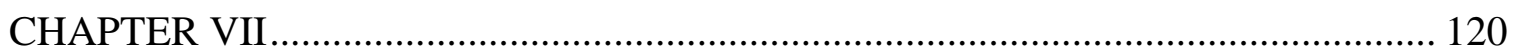

SYNTHESIS AND CHARACTERIZATION OF LAYERED HYBRID PEROVSKITES

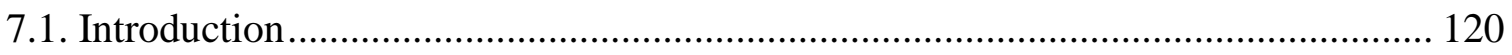

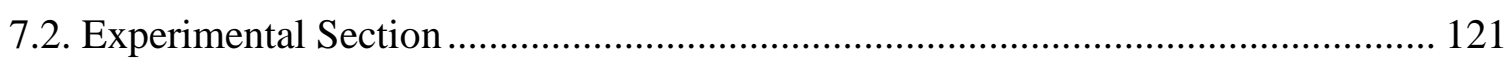

7.2.1. Preparation of Precursor Solution .................................................................. 121

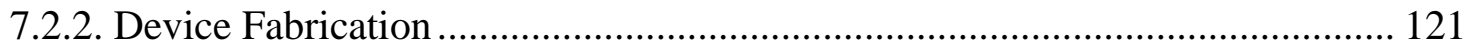

7.2.3. Characterization with AC Photocurrent Method ................................................ 122

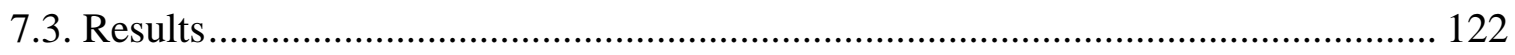

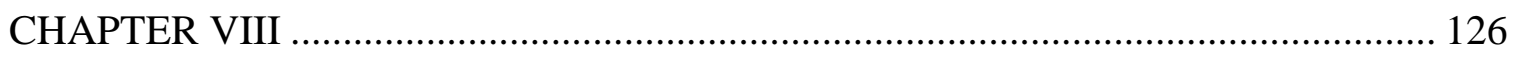

CONCLUSIONS AND FUTURE DIRECTIONS.................................................. 126

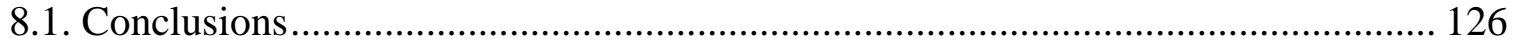

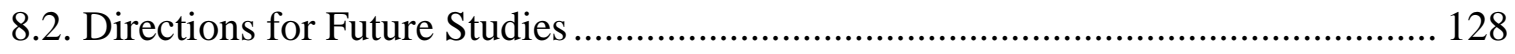

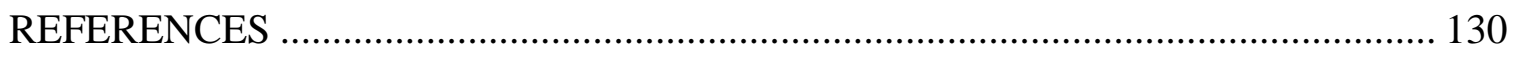

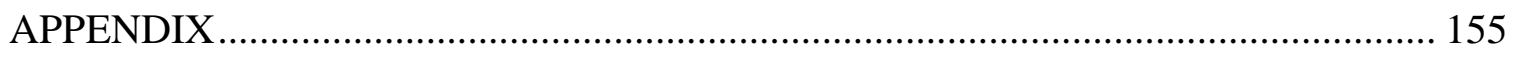

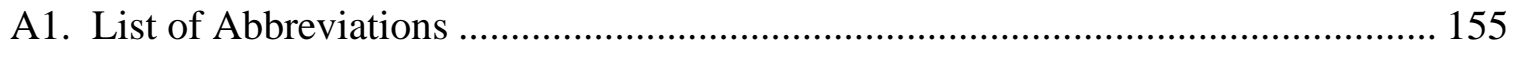

A2. List of Symbols .............................................................................................. 157

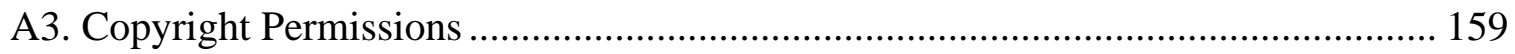

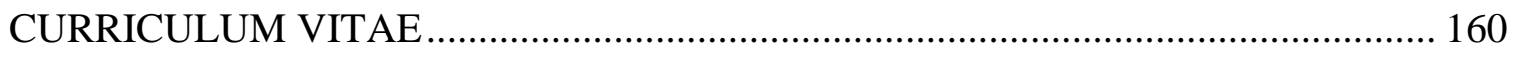




\section{LIST OF TABLES}

Table 1.1. Different ions used in layered hybrid perovskites

Table 2.1. $\mathrm{MACl}$ and n-butylamine for the synthesis of $2 \mathrm{D}$ hybrid perovskite crystals.. 54

Table 3.1. The cell parameters of different batches of traditional, Nd-doped and Er-doped

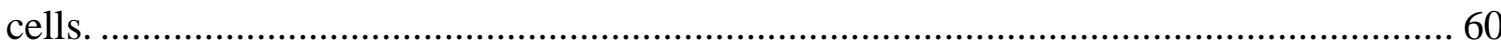

Table 3.2. The cell parameters of cells measured by NREL ....................................... 61

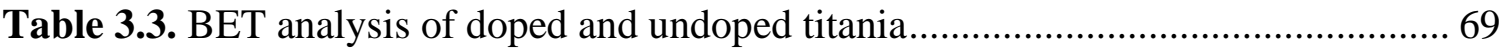

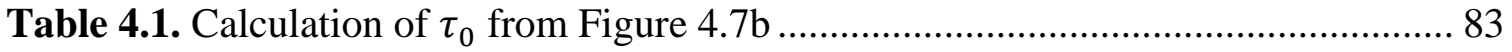

Table 4.2. Parameters extracted from Figure $4.7 \mathrm{~b}$ and $4.9 \mathrm{a}$ for doped and undoped titania. 86

Table 5. 1. Average grain size, measured built-in potential $\left(\mathrm{V}_{\mathrm{bi}}\right)$, and polarization for the different MHHP films used in this study.

Table 6.1. Resonance frequencies $(\mathrm{Hz})$ of QCP in dark and light with different backgrounds 115 


\section{LIST OF FIGURES}

Figure 1.1. (a) p- and n-type of semiconductors brought together, and (b) p-n diode in equilibrium which is equivalent to a typical solar cell.

Figure 1.2. Illustration of working principle of a typical solar cell. ............................. 3

Figure 1.3. A typical current density vs potential curve for a solar cell. ......................... 5

Figure 1.4. Configurations of different generation solar cells: (a) industrial silicon solar cell, (b) copper indium gallium diselenide (CIGS) solar cell, (c) cadmium telluride (CdTe) solar cell, (d) Copper zinc tin sulfide solar cell, (e) organic solar cell, and (f)

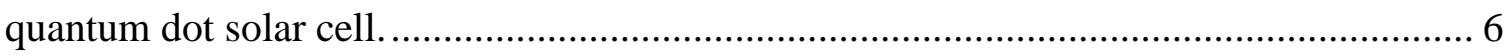

Figure 1.5. Different types of semiconductors used in organic photovoltaics................. 7

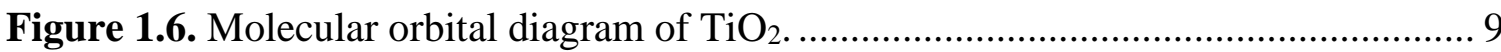

Figure 1.7. Commonly used dyes used in DSSCs...................................................... 10

Figure 1.8. A typical dye-sensitized solar cell showing the path of electrons................ 12

Figure 1.9. Molecular orbital diagram of different components of a DSSC with average time for various electron transfer processes........................................................... 14

Figure 1.10. (a) Unit cell and (b) spatial distribution of A, B and X in a crystal lattice.. 16 Figure 1.11. Parallel plate capacitor. When an electric field is applied, the dielectric gets polarized, which induces opposite charge on the plates. .......................................... 19

Figure 1.12. Architectures of different types of perovskite solar cells. ....................... 21

Figure 1.13. Working principle of a perovskite solar cell with band diagrams. These band diagrams represent a planar n-i-p perovskite solar cell. 
Figure 1.14. The spatial distribution of different cations and anions in the crystals of 2D hybrid perovskites.

Figure 2.1. Temperature ramping program while sintering the films. 31

Figure 2.2. (a) Photoanode sensitized with a dye and (b) counter electrode used in DSSCs. 33

Figure 2.3. Assembling photoanode and counter electrode to make a sandwich DSSC. 35 Figure 2.4. (a)-(c) Vacuum backfilling of electrolyte in a DSSC, (d) a DSSC with sealed

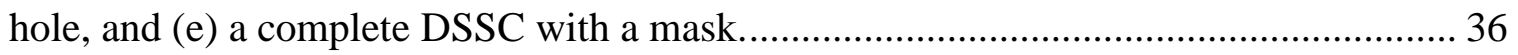

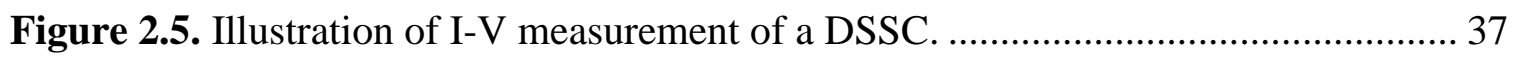

Figure 2.6. Synthesis of methylammonium chloride ............................................... 39

Figure 2.7. Hot casting method for making highly granular films. ............................ 41

Figure 2.8. Shadow mask for the interdigitated contacts......................................... 42

Figure 2.9. Experimental setup for the measurement of (a) AC photocurrent spectrum, and (b) built-in potential and phase. 44

Figure 2.10. ASTM procedure for determining the average size of grains. The average grain size for this sample was found to be $85.22 \mu \mathrm{m}$.

Figure 2.11. Experimental setup for QCM measurement with quartz crystal coated with perovskite film. 48

Figure 2.12. Etching of ITO using zinc paste and $2 \mathrm{M} \mathrm{HCl}$....................................... 50

Figure 2.13. Configuration of perovskite solar cell. ................................................ 50

Figure 2.14. Digital pictures of the (a) mounting of the solar cell on the optical cryostat

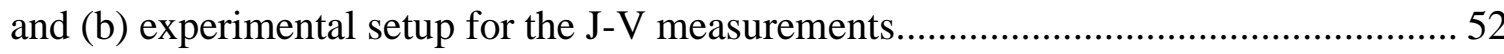

Figure 2.15. Digital picture of crystals of 2D and 3D hybrid perovskites..................... 54

Figure 3.1. (a) JV curves of the best cells and (b) IPCE of cells measured by NREL. All these cells had an approximately $11 \mu \mathrm{m}$ thick mesoporous layer and 4-5 $\mu \mathrm{m}$ scattering layer. 
Figure 3.2. JV plots of cells measured by NREL after 48 hours. The corresponding quantum efficiency plot is given in Figure 3.1b.

Figure 3.3. UV/Vis spectrum of (a) doped and undoped titania on FTO and (b) C101 dye.

Figure 3.4. The parameters of individual cells (a) efficiency, (b) short-circuit current density, (c) open circuit potentials, and (d) fill factors. • : ErNPs-Solid cells, $\bullet$ : NdNPsSolid cells, $\bullet$ : Traditional cells, and $* / * / *$ : corresponding to the best cells. 62

Figure 3.5. Nyquist plot of the cells shown in Figure 3.1a. 63

Figure 3.6. (a) Optimization of concentration of REO nanoparticles for the best photovoltaic performance of DSSCs. These cells had a $7 \mu \mathrm{m}$ thick mesoporous layer and a 4-5 $\mu \mathrm{m}$ thick scattering layer. (b) Dye absorbance as a function of concentration of REO. These photoanodes had a $7 \mu \mathrm{m}$ thick mesoporous film only. 64

Figure 3.7. Optical images (first row) and AFM Image (second row) and 3D images (last row) of doped and undoped photoanodes at the optimized doping levels. 66

Figure 3.8. SEM images of undoped and doped films under optimized conditions. 67

Figure 3.9. (a) $\mathrm{N}_{2}$ gas adsorbed at $\mathrm{STP}$, and (b) BET adsorption isotherms for undoped and doped titania. Here $\mathrm{V}_{\mathrm{a}}$ is the volume of gas adsorbed. 68

Figure 4.1. Experimental setup for the electrochemical measurements of doped and undoped titania.

Figure 4.2. (a) SEM image and (b) illustration of the device used for solid-state conductivity measurements. 74

Figure 4.3. Solid-state conductivity measurements of (a) undoped titania, (b) $\mathrm{Er}_{2} \mathrm{O}_{3}-$ doped titania, and (c) $\mathrm{Nd}_{2} \mathrm{O}_{3}$-doped titania in dark and light as indicated. 76

Figure 4.4. Conductivity measurements of films of pure (a) $\mathrm{Er}_{2} \mathrm{O}_{3}$ and (b) $\mathrm{Nd}_{2} \mathrm{O}_{3}$ nanoparticles. The films of REO NPs are less conductive than undoped titania. 77 Figure 4.5. Cyclic voltammograms of (a) undoped, (b) ErNPs-Solid and (c) NdNPsSolid at different scan rates as shown in the keys. The scan rates are in $\mathrm{mV} / \mathrm{s}$. 78 
Figure 4.6. Cathodic peak positions of (a) undoped, (b) $\mathrm{Er}_{2} \mathrm{O}_{3}$-doped, and (c) $\mathrm{Nd}_{2} \mathrm{O}_{3}-$ doped titania at different scan rates.

Figure 4.7. (a) Boltzmann type exponential factor calculated at the voltage of the deep trap capacitance peak versus scan rate where dots are experimental values and solid lines are linear fits and (b) plot of $V_{p}$ vs natural logarithm of $v$ in $\mathrm{mV} / \mathrm{s}$, where dots are experimental values and solid lines are the linear fits. 83

Figure 4.8. Chronoamperometric curves doped and undoped titania. 84

Figure 4.9. (a) Mott-Schottky plots of doped and undoped titania. Illustration of band bending in (b) undoped and (c) REO-doped .... 86

Figure 4.10. Energy diagram of $\mathrm{TiO}_{2}$ and $\mathrm{REO}\left(\mathrm{Nd}_{2} \mathrm{O}_{3}\right)$. The trap states close to the $\mathrm{VB}$ of $\mathrm{TiO}_{2}$, which are responsible for the degradation in the electrical properties are filled by the REO VB or f-electrons, thereby reducing the capture of $\mathrm{CB}$ electrons of titania....... 88

Figure 5.1. (a) Large grain film of MHHP showing a pair of interdigitated Au contacts (device HP3D1, Table 5.1), (b) and (c) optical images of a highly granular and a small grain films with average grain size $171 \pm 37 \mu \mathrm{m}$ and $29 \pm 5 \mu \mathrm{m}$ respectively. The scale bar in Figure a-c is $100 \mu \mathrm{m}$. (d) and (e) XRD spectra of the films shown in Figure b-c....... 92 Figure 5.2. (a) Experimental setup for $A C$ photocurrent measurement, (b) AC photocurrent spectrum at no external field $(\mathrm{V}=0 \mathrm{mV})$ and at $\mathrm{V}=\mathrm{V}_{\text {bi. }}$. Optical absorbance (blue trace) is shown for comparison. 93

Figure 5.3. (a) Open circuit voltage of the device in the dark and light, and (b) DC photocurrent under various conditions.

Figure 5.4. (a) AC photocurrent as a function of the external potential, which passes through $\mathrm{V}_{\mathrm{bi}}$, and (b)-(d) band diagrams at external potential less than, equal to and more than $\mathrm{V}_{\mathrm{bi}}$, respectively.

Figure 5.5. (a)-(e) Optical images of devices with varying grain size and (f) variation of zero-bias polarization with grain size. The scale bar is $100 \mu \mathrm{m}$ in each image. 97

Figure 5.6. (a) Shift of polarization after poling the device HP3D1 at $5 \mathrm{~V}$ for $1 \mathrm{~h}$ and then relaxation after $1 \mathrm{~h}$. The unit of polarization is $\mu \mathrm{C} \mathrm{cm}^{-2}$. (b)-(e) time dependence of 
polarization of different devices after poling at $5 \mathrm{~V}$ for $1 \mathrm{~h}$. The dashed horizontal line is

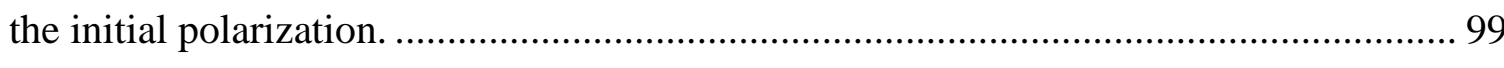

Figure 5.7. Optical images of a small grain film (a) and a large grain film (b) of MHHP, and the corresponding polarization curves (c-d). The black square refers to the polarization of the pristine sample $\left(\mathrm{P}_{0}\right)$ while the red square is an approximate point of

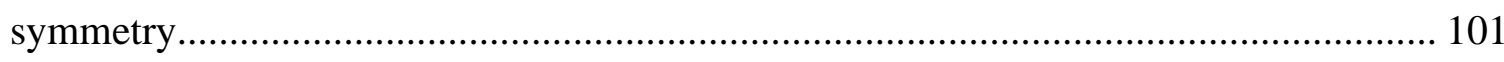

Figure 5.8. Dependence of the electric polarization on the concentration of $\mathrm{PbI}_{2}$....... 102

Figure 6.1. (a) Optical image of a granular film of MHHP with interdigitated gold contacts, (b) experimental setup for AC photocurrent measurements, (c) polarization of

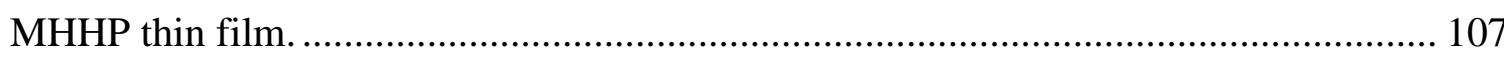

Figure 6.2. Time-dependence of the AC photocurrent in different background environments

Figure 6.3. (a) Experimental setup for AC photocurrent spectrum measurements, and (c) AC photocurrent response in vacuum and Ar gas. 110

Figure 6.4. AC photocurrent spectrum in different inert gases.

Figure 6.5. AC photocurrent spectrum of MHHP film device in air after different times of exposure. 112

Figure 6.6. Changes in the resonant frequency of QCP in dark and light when the background is vacuum (red trace) and Ar (green trace).

Figure 6.7. Changes in the RF of QCP in dark and light when the background gas is He (blue trace) and $\mathrm{N}_{2}$ (green trace). The RF in vacuum (red trace) is shown for comparison.

Figure 6.8. (a) Highly granular film of MHHP film spin-coated by hot-casting, and (b) architecture of perovskite solar cell. 116

Figure 6.9. J-V curves of perovskite solar cell when the cell is exposed to (a) light in vacuum for different times, and (b) Ar gas and vacuum in the dark.

Figure 6.10. J-V curves of perovskite solar cell when the cell is exposed to (a) light under Ar gas environment, and (b) light with evacuating Ar gas for different times..... 117 
Figure 6.11. Variation of short-circuit current density and efficiency under the different

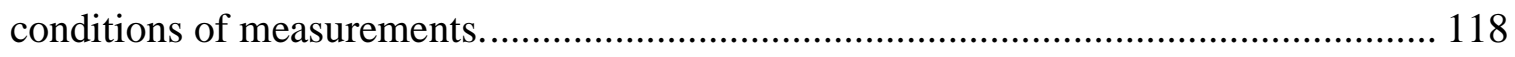

Figure 7.1. (a)-(d) Optical images of the films of different 2D hybrid perovskites, and (e) low angle XRD spectra of these perovskites spin-coated on the glass substrates......... 123

Figure 7.2. Optical absorbance spectra of different types of 2D hybrid perovskites.... 124 Figure 7.3. (a) Optical image of the film for $n=4$ with interdigitated Au contacts used for the AC photocurrent measurement, (b)-(d) the AC photocurrent spectra of the device for

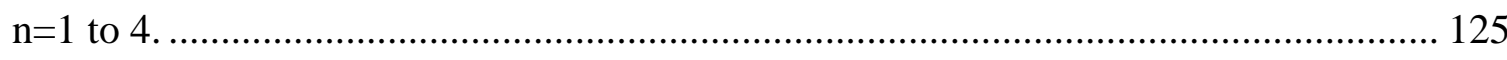




\section{LIST OF SCHEMES}

Scheme 2.1. Preparation of photoanodes for DSSCs................................................ 32

Scheme 2.2. Fabrication of photoanodes for best performing DSSCs.......................... 32

Scheme 2.3. Fabrication of counter electrodes used in DSSCs.................................... 34

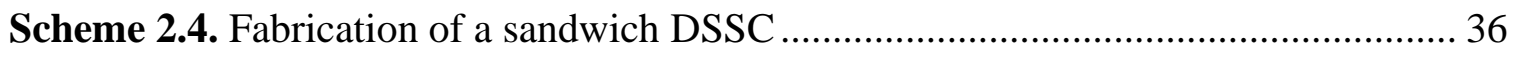

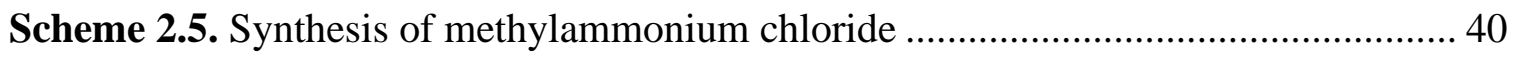

Scheme 2.6. Device fabrication for AC photocurrent measurements. ........................... 42

Scheme 2.7. Built-in potential measurements in different environments. ..................... 46

Scheme 2.8. Fabrication of perovskite solar cell ................................................. 51

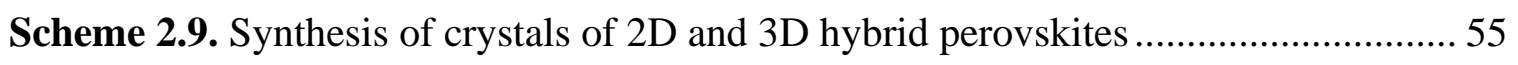

Scheme 7.1. Fabrication of devices for AC photocurrent measurements. .................... 122 


\section{CHAPTER I}

\section{INTRODUCTION}

\subsection{Demand of Renewable Sources of Energy}

The total population on earth has reached over 7.8 billion and is projected to exceed 9.7 billion by 2050 and 10.9 billion by $2100 .{ }^{1-2}$ The global energy consumption is also increasing in the same proportion. Considering the present status of population growth and energy consumption, the global demand is expected to double by 2050 and quadruple by the end of the century. ${ }^{3}$ A high percentage ( 93\%) of this energy demand is fulfilled by non-renewable sources of energy, and the storage of these sources is limited. The current estimation of global reserves for fossil fuels, natural gas, and coal suggests that the fossil fuels have the potential to provide continuous energy for up to 54 years, natural gas up to 63 years, and coal up to 107 years. ${ }^{4-5}$ Renewable and clean energy is expected to replace non-renewable sources of energy for satisfying the energy demand of humankind. ${ }^{6}$ The available renewable sources of energy are solar, wind, bio-fuels, hydropower, geothermal, and ocean. Nevertheless, carbon-free energy resources are crucial for the sustainable development of global society without degradation of the environment. The sun delivers $1.2 \times 10^{5} \mathrm{TW}$ energy per year to the earth. Only $0.5 \%$ ( 600 TW) of this energy can be practically converted to electrical energy. Reports reveal that even $10 \%$ of this energy is enough to compensate the global energy demand of future generations. ${ }^{7-8}$ Thus, the development and use of devices which convert solar energy to a usable form of energy are crucial for the conservation of the non-renewable sources of energy. 


\subsection{Solar Cells}

A solar cell is a device that directly converts the energy from sunlight into electrical energy through the photovoltaic effect. It is also known as a photovoltaic cell.

\subsubsection{Architecture and Working Principle of Solar Cells}

A solar cell consists of an assembly of a layer of p-type semiconductor placed next to a layer of n-type semiconductor. There are excess of electrons in the n-type layer and excess of positively charged holes in the p-type layer, which resembles a p-n diode. When these layers are not in contact, the fermi level of the n-type semiconductor is close to the conduction band, and that of p-type semiconductor is close to the valence band as shown in Figure 1.1a. On bringing these layers into contact, some of the electrons diffuse from the $\mathrm{n}$-type region to the p-type region while holes diffuse from the p-type region to the n-type region. As a result, the fermi level of the n-type semiconductor decreases while the fermi level of the p-type semiconductor increases, until finally they have the same fermi level at equilibrium as shown in Figure 1.1b. Near the junction of the two layers, the electrons on one side of the junction (n-type layer) combine with the holes on the other side of the junction (p-type layer). This creates an area around the junction where the carriers are depleted. This is called the depletion zone. When all the holes are filled with electrons in the depletion zone, the p-type side of the depletion zone (where holes were initially present) now contains uncompensated negatively charged ions, and the n-type side of the depletion zone (where electrons were present) now contains uncompensated positively charged ions. The presence of these oppositely charged ions creates an internal electric field. This internal field is called the built-in potential $\left(\mathrm{V}_{\mathrm{bi}}\right)$. The $\mathrm{V}_{\mathrm{bi}}$ acts an an energy barrier that prevents free movement of charge carriers, or electrons in the n-type layer from filling holes in the p-type layer. 

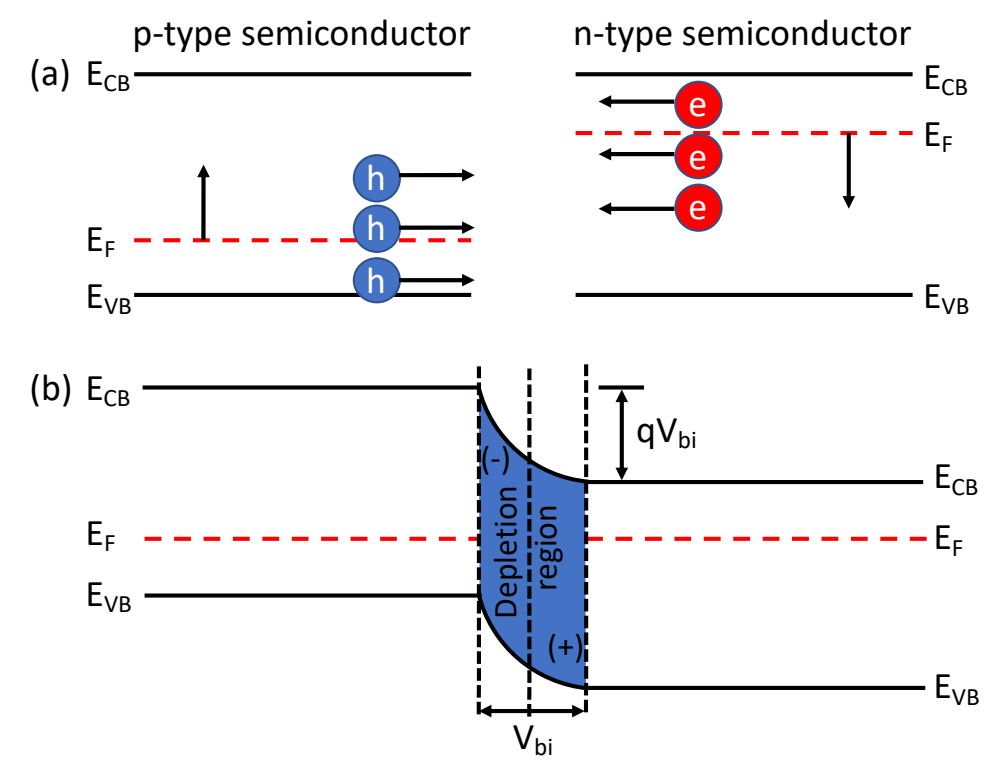

Figure 1.1. (a) p- and n-type of semiconductors brought together, and (b) p-n diode in equilibrium which is equivalent to a typical solar cell.

When sunlight is allowed to fall on a solar cell, the electrons in the semiconductor are excited to the conduction band, and it results in the formation of "holes", the vacancies left behind by the escaping electrons. The internal electric field helps in the separation of these electron-hole pairs. If this happens in the electric field, the field will move electrons to the n-type layer and holes to the p-type layer. If we connect the n-type and p-type layers with a metallic wire, the electrons will travel from the n-type layer to the p-type layer through the external wire, creating a flow of electricity as shown in Figure 1.2.

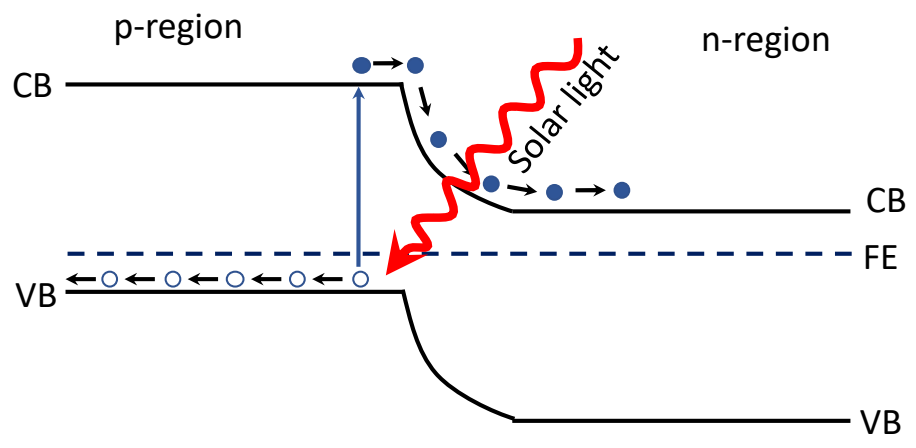

Figure 1.2. Illustration of working principle of a typical solar cell. 


\subsubsection{Photoelectrochemical Parameters of Solar Cells}

The photoelectrochemical parameters are the terms used to explain the performance

of a solar cell. Basically, the following five photoelectrochemical (PEC) parameters are used to explain the performance of a solar cell:

(a) Open-circuit voltage. When the cell is operated at open circuit (no current flowing through the cell), the potential difference across the output terminals is defined as the open-circuit voltage. It is denoted by $\mathrm{V}_{\text {OC. }}$ It is marked by $\mathrm{V}_{\text {OC }}$ in a typical current density-potential curve in Figure 1.3.

(b) Short-circuit current density. When a cell is operated at short circuit condition, the current density through the terminals of the cell, when the light at one sun is shone on the cell, is defined as the short-circuit current density. It is denoted by JSC (Figure $1.3)$.

(c) Fill factor. Fill factor (FF) is the ratio of the maximum electrical power $\left(\mathrm{P}_{\max }\right)$ dissipated by a cell to the product of $\mathrm{J}_{\mathrm{SC}}$ and $\mathrm{V}_{\mathrm{OC}}$. In Figure 1.3., FF is the ratio of the area of blue rectangle to the area of yellow rectangle. Mathematically, FF can be expressed as

$$
\mathrm{FF}=\frac{\mathrm{P}_{\max }}{\mathrm{J}_{\mathrm{sc}} \cdot \mathrm{V}_{\mathrm{oc}}}=\frac{\mathrm{J}_{\max } \cdot V_{\max }}{\mathrm{J}_{\mathrm{sc}} \cdot \mathrm{V}_{\mathrm{oc}}}
$$

where $\mathrm{J}_{\max }$ and $\mathrm{V}_{\max }$ are the values of current density and potential corresponding to $\mathrm{P}_{\max }$ as shown in Figure 1.3.

(d) Efficiency. The efficiency ( $\eta$ ) of a solar cell is the ratio of the maximum power $\left(\mathrm{P}_{\text {out }}\right)$ dissipated by a cell to the power of incident light $\left(\mathrm{P}_{\text {in }}\right)$ on it. Mathematically, $\eta$ can be expressed as:

$$
\eta=\frac{P_{\text {out }}}{P_{\text {in }}} \times 100 \%=\frac{J_{S C} \times V_{O C} \times F F}{P_{\text {in }}} \times 100 \% .
$$




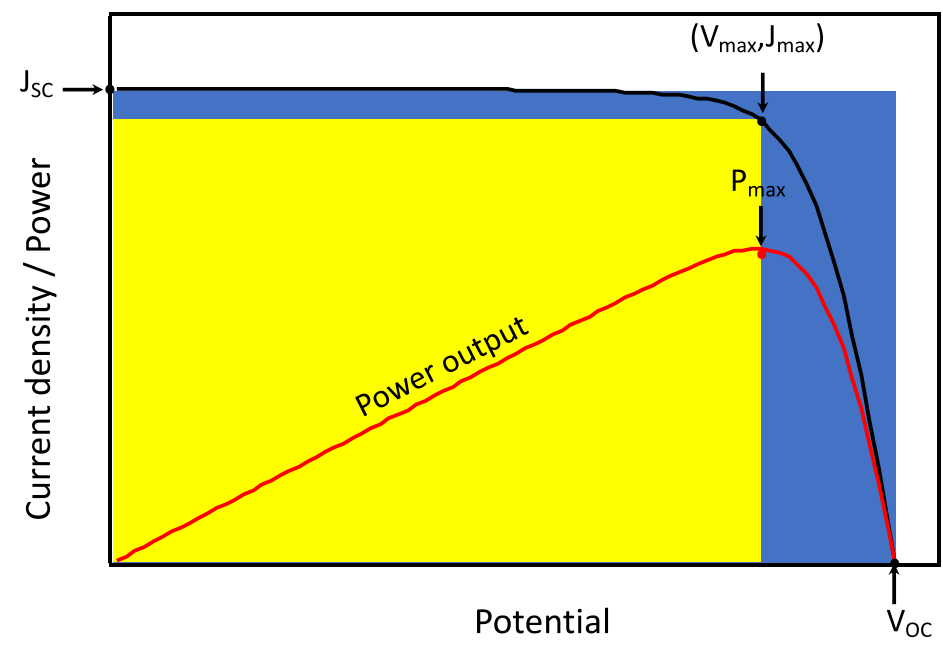

Figure 1.3. A typical current density vs potential curve for a solar cell.

(e) Incident photon-to-electron conversion efficiency. The number of photo electrons generated per unit photon absorbed by a solar cell is known as its incident photonto-electron conversion efficiency (IPCE). IPCE is also known as external quantum efficiency (EQE). The photocurrent density observed from a solar cell at different wavelengths of solar light incident on the cell can be used to get EQE by

$$
\mathrm{EQE} \%=\frac{\mathrm{J}_{\mathrm{SC}}\left(\mathrm{A} \mathrm{cm}^{-2}\right)}{\mathrm{P}\left(\mathrm{W} \mathrm{cm} \mathrm{cm}^{-2}\right)} \times \frac{1240}{\lambda(\mathrm{nm})} \times 100
$$

EQE directly affects the photoconversion efficiency of a solar cell.

\subsubsection{Semiconductors Used in Different Generation Solar Cells}

A solar cell uses a semiconductor as a light-harvesting material to convert solar energy into electrical energy. The material undergoes photovoltaic effect for the energy conversion. It does so by generating electron-hole pairs upon the absorption of photons of suitable energy. Different types of semiconductors are used in different types of solar cells. Silicon-based photovoltaics, the first-generation solar cells are the commercially available solar cells. ${ }^{9-11}$ In these solar cells p-doped and n-doped silicon semiconductors are used. The scheme of a typical industrial silicon solar cell is depicted in Figure 1.4a. Second generation solar cells are usually called thin-film solar cells because they are made up of 
layers of semiconductors only a few microns thick. Basically, there are three types of solar cells that are considered in this category, amorphous silicon and two that are made from non-silicon materials namely copper indium gallium diselenide (CIGS), which uses CIGS, $\mathrm{CuIn}_{(1-\mathrm{x})} \mathrm{Ga}_{\mathrm{x}} \mathrm{Se}_{2}$ of band gap 1 to $1.7 \mathrm{eV}$ for $\mathrm{x}=0-1$ and $\mathrm{ZnO}$ of band gap $3.37 \mathrm{eV}$ (Figure 1.4b), and cadmium telluride (CdTe) solar cell (Figure 1.4c) which uses two semiconductors $\mathrm{CdS}$ of bandgap $2.42 \mathrm{eV}$ and CdTe of bandgap $1.5 \mathrm{eV}$. Third-generation solar cells are the solar cells that are potentially able to overcome the Shockley-Queisser limit of $31-41 \%$ power efficiency for single bandgap solar cells. Emerging third generation photovoltaics include: Copper zinc tin sulfide solar cell (CZTS), ${ }^{12}$ organic solar cells (OSCs) ${ }^{13}$ quantum dot solar cells (QDSCs), ${ }^{14}$ dye-sensitized solar cells (DSSCs),${ }^{15}$ and perovskite solar cells (PSCs). ${ }^{16} \mathrm{CZST}$ solar cell (Figure 1.4d) uses copper zinc tin sulfide with bandgap of 1.4-1.5 eV as a light absorber. ${ }^{12}$ OSCs use conjugated, semiconducting polymers and small organic molecules as shown in Figure $1.5 \cdot{ }^{13} \mathrm{~A}$ typical OSC is shown in Figure 1.4e. Quantum dots are used as the light-harvesting material in QDSCs (Figure 1.4f). DSSCs, also known as "Grätzel cells", are explained in section 1.3 while perovskite solar cells in section 1.4.

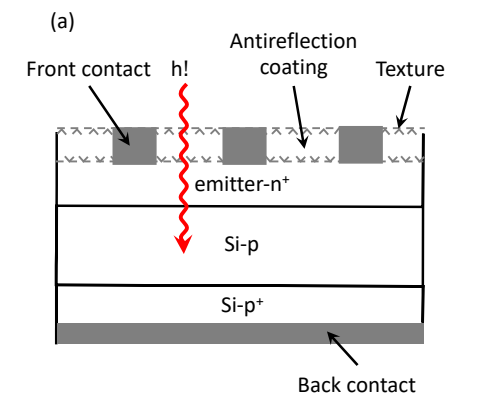

(b)
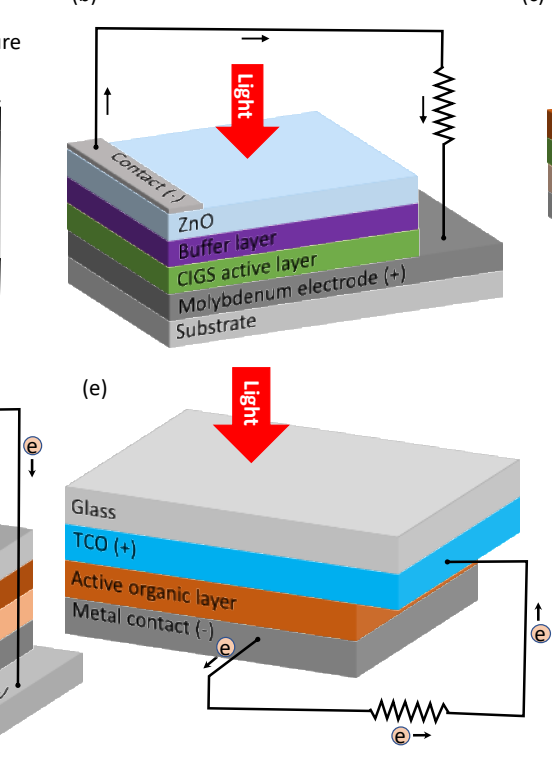

(c)
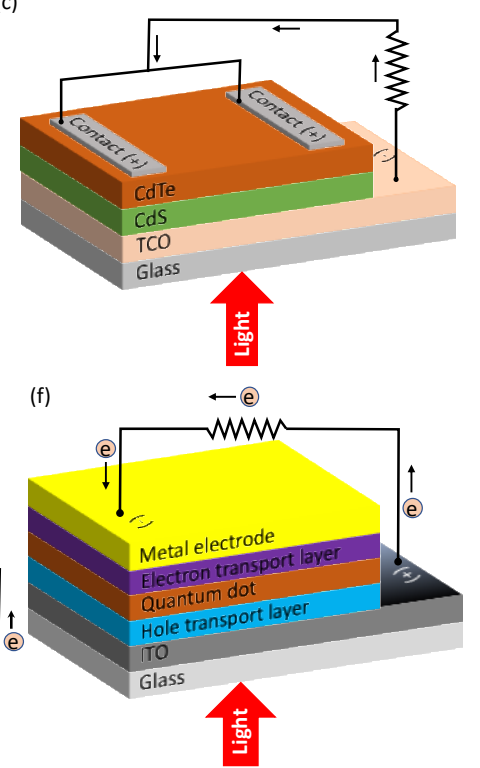

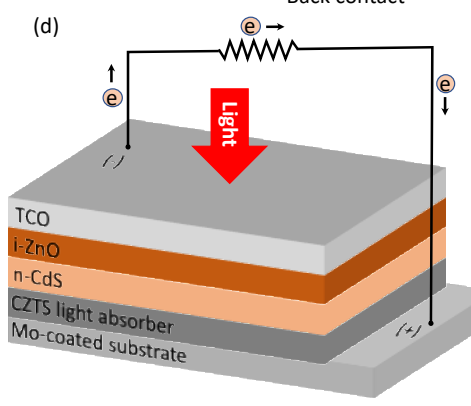

Figure 1.4. Configurations of different generation solar cells: (a) industrial silicon solar cell, (b) copper indium gallium diselenide (CIGS) solar cell, (c) cadmium telluride (CdTe) solar cell, (d) Copper zinc tin sulfide solar cell, (e) organic solar cell, and (f) quantum dot solar cell. 


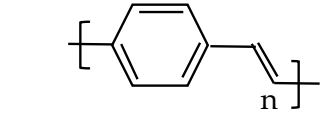

Poly(phenylene vinylene) (PPV)

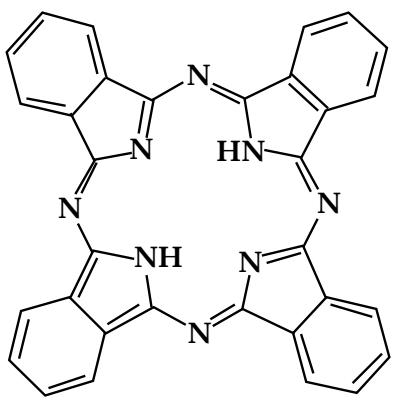

Phthalocyanine

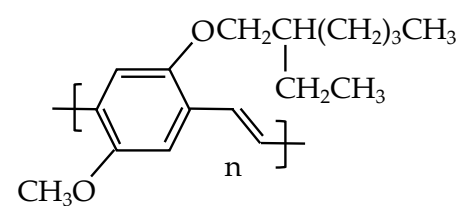

MEH-PPV

Polyacetylene

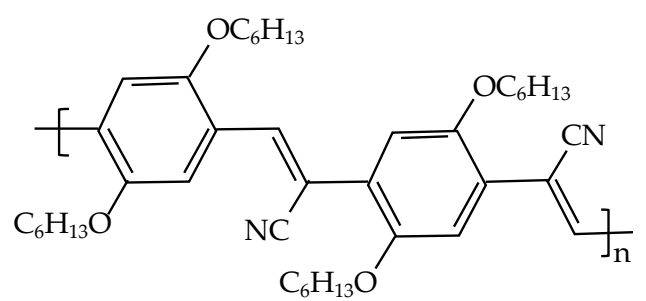

CN-PPV

Figure 1.5. Different types of semiconductors used in organic photovoltaics.

\subsection{Dye-Sensitized Solar Cells}

A dye-sensitized solar cell uses the principle of photosynthesis to convert solar energy to electrical energy, and was first developed in 1991 by O'Regan and Grätzel. ${ }^{17}$ It is a simple photovoltaic cell that has the potential to harvest solar energy at a low manufacturing cost with low environmental impact. It is considered to be a very promising photovoltaic technology since it offers many exclusive features such as semi-transparency, flexibility and lightweight applications, and also good performance under low light conditions and different solar incident angles. ${ }^{18-20}$

\subsubsection{Components of Dye-Sensitized Solar Cells}

A DSSC is composed of mainly five components: a transparent conducting substrate, a thin film of wide-band gap semiconductor, a photosensitizer adsorbed on the film of 
semiconductor, a redox electrolyte and a counter electrode. Each of these components are explained below:

(a) Transparent conducting substrate. A transparent conducting oxide (TCO) coated substrate is commonly used as a substrate for DSSC fabrication because of its relatively low cost, abundance, and high optical transparency in the visible and infrared region of the solar spectrum. The commonly used TCO substrates are fluorine-doped tin oxide (FTO) coated glass, and indium tin oxide (ITO) coated glass. FTO glass is preferred over ITO glass in DSSCs because of its better thermal stability at high temperature.

(b) Suitable semiconductor. One of the most investigated components in DSSCs is the mesoporous semiconducting layer which provides the surface for the adsorption of photo-absorber molecules (dyes) and plays a significant role for efficient charge separation leading to the photo-injection of charge carriers. ${ }^{21}$ Titanium dioxide,${ }^{22-23}$ zinc oxide, ${ }^{24-26}$ ferric oxide, ${ }^{27}$ niobium pentoxide,${ }^{28}$ cerium (IV) oxide,${ }^{29}$ tin (IV) oxide, ${ }^{30}$ strontium titanate ${ }^{31}$ barium stannate, ${ }^{32}$ and zinc stannate ${ }^{33}$ are commonly used semiconductors in DSSCs. Titanium dioxide (titania) is a widely investigated semiconductor in DSSCs. Of the two modifications: anatase and rutile of titania, the anatase modification is preferred for the use in photovoltaic applications as it has been shown to have better adsorption of dye and electron transport properties. ${ }^{34}$ Other advantages using this semiconductor are large bandgap $(\sim 3.2 \mathrm{eV})$, high conduction band edge energy, chemical and mechanical stability, efficient electron-accepting capability, and high redox activity in the illuminated condition. ${ }^{22}$ An efficiency over $14 \%$ has been reported with this semiconductor. ${ }^{35}$ The molecular orbital picture of titania is shown in Figure 1.6. 


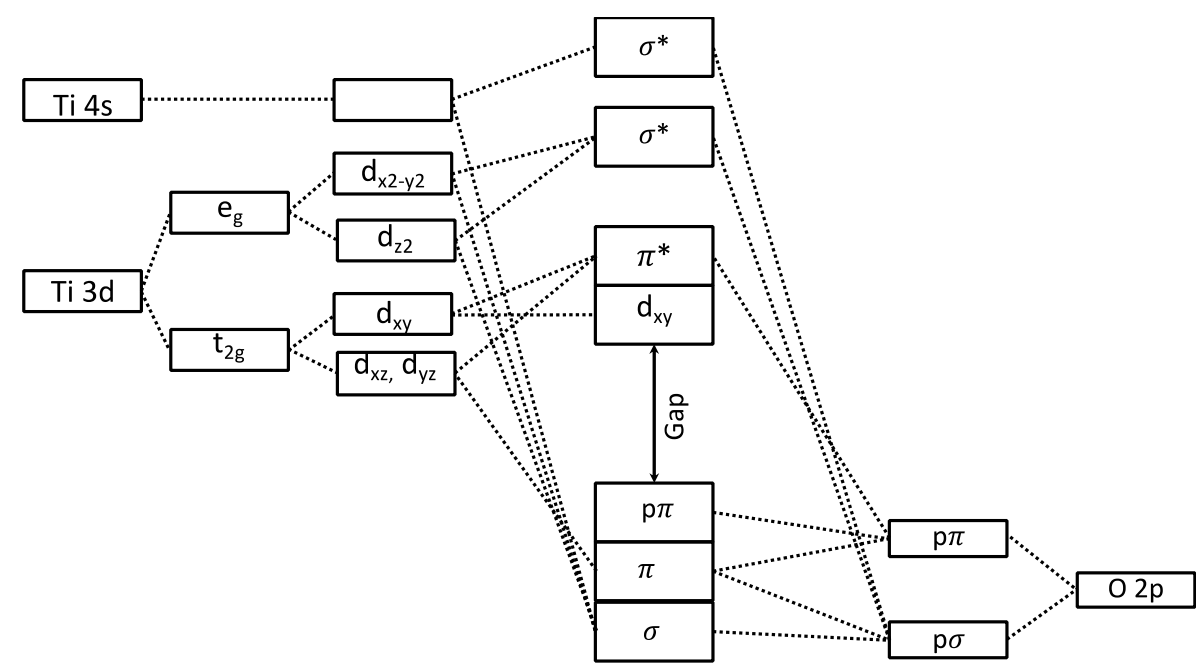

Figure 1.6. Molecular orbital diagram of $\mathrm{TiO}_{2}$.

(c) Photosensitizer. A photosensitizer, also known as dye, is a chief part of a DSSC that absorbs and converts solar energy into electrical energy. A good dye for DSSCs is one which has (i) a wide panchromatic capacity to harvest light to produce a large current, (ii) high molar extinction coefficient to excite as many electrons as possible, molecular orbitals that match well with the host material conduction band for efficient charge injection, (iii) good chemical properties to make a stable monolayer on the host material, (iv) a low HOMO energy level that can be regenerated with electrolyte, and (v) an anchoring group which can bind itself with the nanostructured film. Ruthenium-based metal complexes have shown the best photovoltaic properties including a high panchromatic capacity, suitable excited and ground state energy levels, relatively long excited-state life-time, and good electrochemical stability. ${ }^{36}$ Among Ru based dyes, N3, N719 and C101 are considered as reference dyes for DSSCs. Figure 1.7 shows some of the current dyes used in DSSC technology. ${ }^{37}$ In this work C101 dye was used as the photosensitizer. 


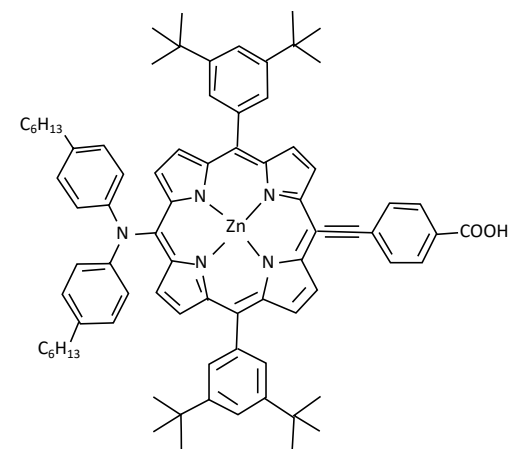

Zinc porphyrin dye

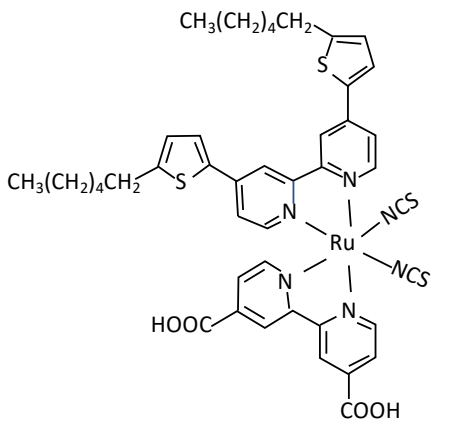

C101 dye

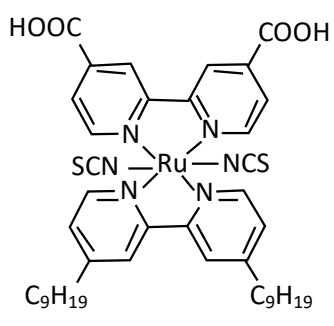

Z907 dye

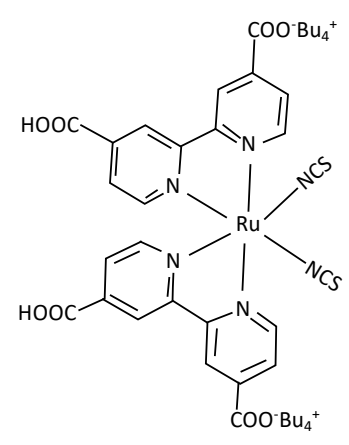

N719 dye

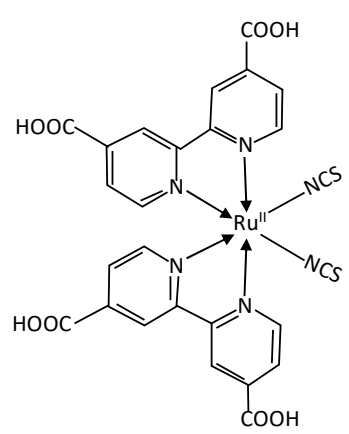

N3 dye

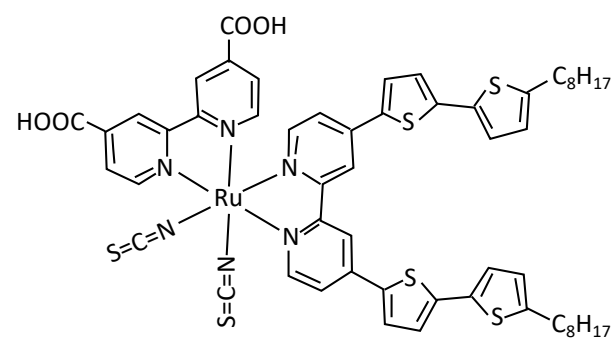

Z991 dye

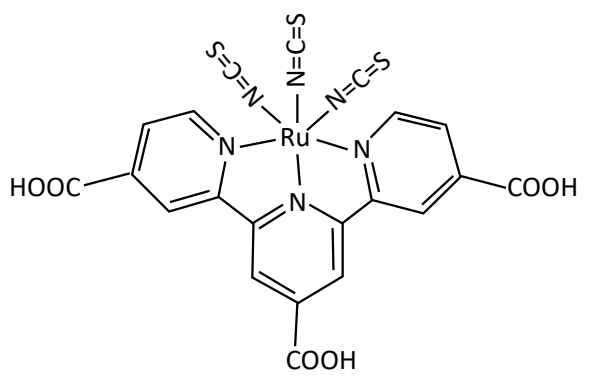

N749 dye

Figure 1.7. Commonly used dyes used in DSSCs.

(d) Electrolyte. An electrolyte that is used in a DSSC has a redox couple to transport holes and plays an important role to regenerate the dye. The redox couples used in the electrolyte are copper complexes, $\mathrm{SeCN}^{-} /(\mathrm{SeCN})_{3}{ }^{-}, \mathrm{SCN}^{-} /(\mathrm{SCN})_{3}{ }^{-}, 3 \mathrm{Br}^{-} / \mathrm{Br}_{3}{ }^{-}, \mathrm{Co}^{2+} / \mathrm{Co}^{3+}$, and $\mathrm{Fc} / \mathrm{Fc}+{ }^{38}$ The most commonly used electrolyte contains the redox couple $3 \mathrm{I}^{-} / \mathrm{I}_{3}{ }^{-}$in an organic solvent. The electrolyte $\mathrm{Z} 960$ containing $3 \mathrm{I}^{-} / \mathrm{I}_{3}{ }^{-}$redox couple in nitrile-based solvent has been found to be a stable electrolyte. ${ }^{39}$ The composition of Z960 electrolyte is 1.0 M 1,3-dimethylimidazolium iodide, $50 \mathrm{mM}$ LiI, $30 \mathrm{mM} \mathrm{I}$, $0.5 \mathrm{M}$ tert-butylpyridine 
and 0.1 M guanidinium thiocyanate in a 17:3 mixture of acetonitrile and valeronitrile by volume. Z960 electrolyte was used for the work described in this dissertation.

(e) Counter electrode. The counter electrode works as a contact for the cathode and also acts as a catalyst for the reduction of the redox couple in the electrolyte. TCO glass coated with a suitable material for catalytic reduction of the electrolyte is used as a counter

electrode. ${ }^{40}$ Carbon (powder and nanotubes), cobalt sulfide and conductive polymers and Pt are commonly coated on the counter electrode. ${ }^{40-41}$ We have used Pt-coated FTO glass as a counter electrode in our work.

\subsubsection{Working Principle of Dye-Sensitized Solar Cell}

A typical $\mathrm{TiO}_{2}$ based DSSC showing a photoanode (PA) made up of a $\mathrm{TiO}_{2}$ film sensitized with dye, a redox electrolyte $\left(3 \mathrm{I}^{-} / \mathrm{I}_{3}{ }^{-}\right)$and a Pt counter electrode (CE) is shown in Figure 1.8. When the sunlight is allowed to fall on the cell, the photons are absorbed by the dye, which is adsorbed on the $\mathrm{TiO}_{2}$ nanoparticle film. The photons cause the excitation of electrons from the highest occupied molecular orbital (HOMO) level to the lowest unoccupied molecular orbital (LUMO) energy level of the dye. Since the conduction band (CB) of $\mathrm{TiO}_{2}$ is in close proximity to the LUMO of the dye, ultrafast electron transfer $(<30$ fs) occurs from the dye LUMO to the $\mathrm{CB}$ of $\mathrm{TiO}_{2}$. These injected electrons diffuse through the mesoporous $\mathrm{TiO}_{2}$ nanoparticles and flow through the external circuit to the $\mathrm{CE}$. The reduction of $\mathrm{I}_{3}^{-}$to $3 \mathrm{I}^{-}$takes place at the $\mathrm{CE}$. The reduced form of the electrolyte $\left(3 \mathrm{I}^{-}\right)$then regenerates the dye by oxidation back to $\mathrm{I}_{3}{ }^{-}$. This cycle continues and the cell keeps generating electricity. 


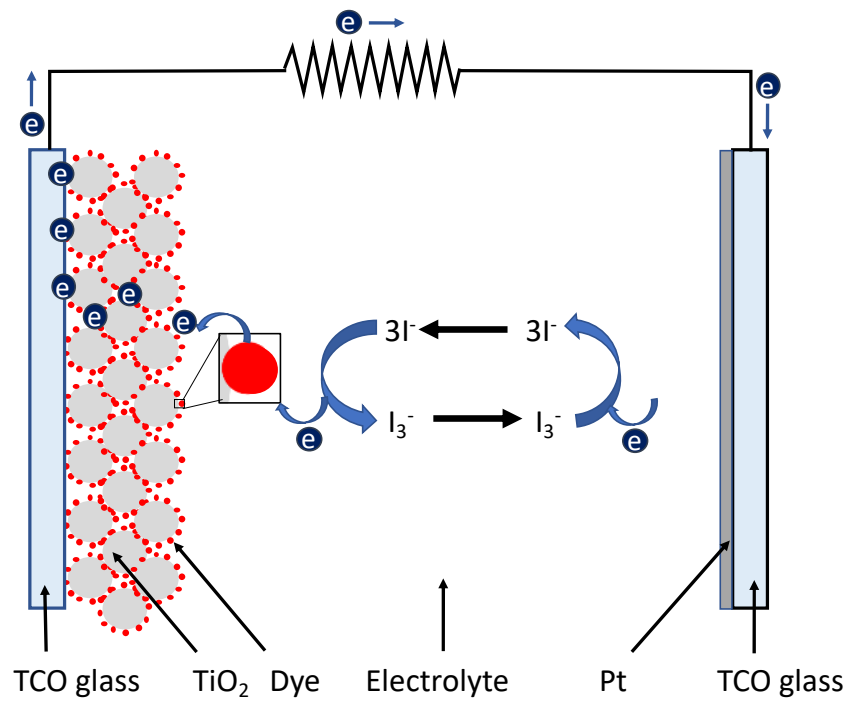

Figure 1.8. A typical dye-sensitized solar cell showing the path of electrons.

The electron-transfer process during the working of a DSSC can be explained as follows:

(a) Photoexcitation of dye. When light falls on the dye molecules, the electrons get excited from VB to $\mathrm{CB}$ of the dye. This process is known as photoexcitation and is depicted in Eq. 1.

$$
\mathrm{S}+\mathrm{hv} \rightarrow \mathrm{S}^{*} \ldots(1)
$$

(b) Injection of electrons. After photoexcitation, the molecules in the excited state decay back to the ground state by emission or undergo oxidative quenching, and the photogenerated electrons are injected to $\mathrm{CB}$ of $\mathrm{TiO}_{2}$. The process of photo injection is shown in Eq. 2. This process is ultrafast and occurs in less than $30 \mathrm{fs}$.

$$
2 \mathrm{~S}^{*}+\mathrm{TiO}_{2}(\mathrm{CB}) \rightarrow 2 \mathrm{~S}^{+}+\mathrm{TiO}_{2}(2 \mathrm{e} \mathrm{CB}) \ldots(2)
$$

(c) Diffusion of electrons. The electrons injected from the dye to $\mathrm{TiO}_{2}$ then diffuse through the mesoporous network of $\mathrm{TiO}_{2}$ nanoparticles (NPs) to reach the FTO collector electrode and flow through the external circuit to the Pt CE. 
(d) Reduction of electrolyte. The electrons reach the CE through the external circuit and reduce triiodide $\left(\mathrm{I}_{3}^{-}\right)$in the solution to iodide $\left(\mathrm{I}^{-}\right)$. The electrochemical reduction of the electrolyte is shown in Eq. 3.

$$
\mathrm{I}_{3}^{-}+2 \mathrm{e} \rightarrow 3 \mathrm{I}^{-} \ldots(3)
$$

(e) Regeneration of dye. $\mathrm{I}^{-}$ions then reduce the oxidized dye, which is the regeneration of dye. This is shown in Eq. 4.

$$
2 \mathrm{~S}^{+}+3 \mathrm{I}^{-} \rightarrow 2 \mathrm{~S}+\mathrm{I}_{3}^{-} \ldots(4)
$$

These processes repeat to convert sunlight into electrical energy. Notably, there are several competing undesirable pathways where the photogenerated electrons are lost during the working of the cell. The pathways for the recombination of electrons are detrimental for the photovoltaic performance. The recombination processes are influenced by the time taken for each of the five processes which occur during the working of a DSSC. These are shown in Figure 1.9. After photoexcitation, there is a possibility that the electrons combine with the oxidized dye and go to the ground state with radiation emission as shown in Eq. 5. This process reduces the electric current directly. The recombination is also possible with the electrons injected into mesoporous $\mathrm{TiO}_{2}$ film. The injected electrons can recombine with the dye or directly reduce $\mathrm{I}_{3}{ }^{-}$ions in the electrolyte instead of going through the external circuit (Eq. 6 and 7). The electrons on the back contact of TCO may also recombine with the electrolyte (Eq. 8), which also degrades the performance of the device.

$$
\begin{aligned}
& \mathrm{S}^{*} \rightarrow \mathrm{S}+\mathrm{h} \nu \ldots(5) \\
& 2 \mathrm{~S}^{+}+2 \mathrm{e}\left(\mathrm{TiO}_{2} \mathrm{CB}\right) \rightarrow 2 \mathrm{~S} \ldots(6) \\
& \mathrm{I}_{3}^{-}+2 \mathrm{e}\left(\mathrm{TiO}_{2} \mathrm{CB}\right) \rightarrow 3 \mathrm{I}^{-} \ldots(7) \\
& \mathrm{I}_{3}^{-}+2 \mathrm{e}(\mathrm{FTO}) \rightarrow 3 \mathrm{I}^{-} \ldots(8)
\end{aligned}
$$




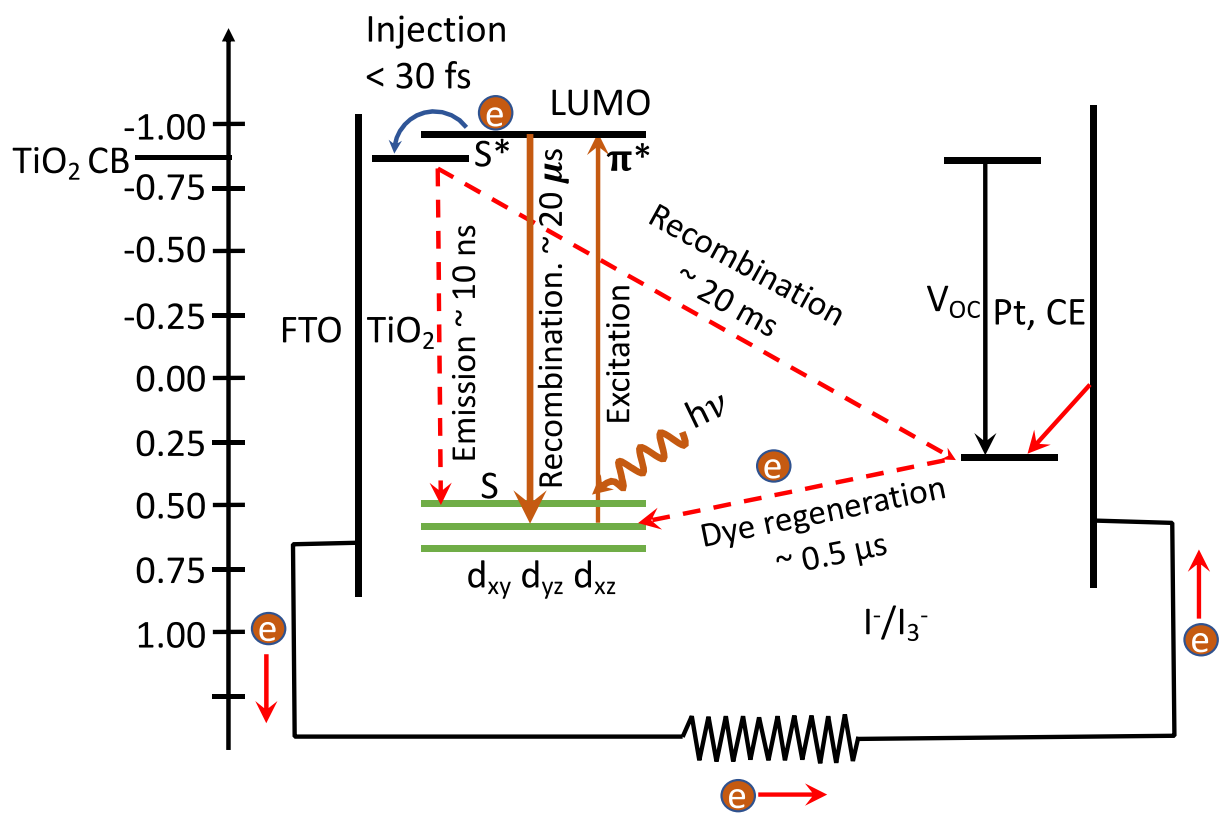

Figure 1.9. Molecular orbital diagram of different components of a DSSC with average time for various electron transfer processes.

\subsubsection{Photovoltaic Performance of Dye-Sensitized Solar Cells and Challenges}

The photovoltaic performance and stability of a DSSC depends on the semiconductor used in mesoporous layer, the dopant in the semiconductor, the type of dye, and the electrolyte. Five parameters namely $\mathrm{J}_{\mathrm{SC}}, \mathrm{V}_{\mathrm{OC}}$, IPCE, $\eta$ and FF are used to compare the performance of different DSSCs. The photocurrent density is the total current produced by a solar cell per unit area of photoanode exposed to solar energy at one sun. It is the sum of the photocurrent density arising due to the total charge injection $\left(\mathrm{J}_{\mathrm{inj}}\right)$ from dye to $\mathrm{TiO}_{2}$ and dark current density $\left(\mathrm{J}_{\text {dark }}\right)$. $\mathrm{J}_{\text {dark }}$ is the opposite current density developed in the device by the recombination of electrons by electrolyte and dye. ${ }^{42}$ The reduction of dark current is very important for minimizing the recombination. The photocurrent density can be increased by using a dye with a broader adsorption spectrum, or by increasing the amount of dye adsorbed onto the mesoporous $\mathrm{TiO}_{2}$, and using suitable dopants for titania. The FF can be increased by careful device design for decreasing the total series resistance of the cell. $V_{O C}$ can be increased by increasing the rate of electron injection or decreasing the 
recombination ${ }^{43}$ or by making the electrolyte potential more positive using different redox couples or using additives. ${ }^{39}$ The improvements in the electrical and physical properties of the $\mathrm{TiO}_{2}$ nanoparticle acceptor, and also the stability of the cells should be taken into consideration for large scale implementation. Studies have been made by the modification of titania with a molecular linker for greater stability, forming core/shell structures by coating with another metal oxide for improvement of all the parameters, doping titania with different metals oxides and metal ions for increasing $\mathrm{J}_{\mathrm{SC}}$ and $\mathrm{V}_{\mathrm{OC}}$, metal nanoparticles, redox inactive ionic species for enhancing the charge mobility, and other dopants for improvement in $\mathrm{J}_{\mathrm{SC}}{ }^{44-50}$

Although the maximum efficiency obtainable for DSSCs is greater than 20\%, a maximum efficiency of $12.3 \%$ has been certified by NREL till date. ${ }^{51}$ It can be said that the efficiency of a DSSC can still be improved.

\subsubsection{Motivation for the Study of Dye-Sensitized Solar Cells}

The current status of energy consumption shows that energy demand requires the consumption of easily available energy resources such as fossil fuels, hydroelectricity, and chemical energy from batteries. Most of these are non-renewable sources, and there are severe environmental issues, such as global warming, and chemical pollutions resulting from the byproducts of fossil fuels. As a result, eco-friendly renewable energy resources are needed as a practical solution to resolve the issues related to environmental concerns and to preserve the existing non-renewable energy sources to push the possible energy crisis into the future. Solar energy is the most practical solution. Due to the high fabrication cost for robust silicon solar technology, there is a need of low-cost solar cells. Dyesensitized solar cell technology has emerged as an alternative to replace silicon technology as it involves low fabrication cost, ability to work in diffuse light, and its ability to produce flexible devices that can be applicable everywhere. However, it has several challenges that have still not been addressed completely. The main challenges are the optimization of efficiency to beyond $20 \%$ for commercialization, the production of chemically stable photoanodes for up to 20 years, and the more complete understanding of charge transfer dynamics within DSSCs, especially at the dye/semiconductor interfaces. It has been shown 
that doping titania with rare-earth metal oxide (REO) microparticles led to the photovoltaic enhancement of DSSCs drastically. ${ }^{52}$ The nanoparticles have very high surface to volume ratios and are highly effective in catalytic activities because of larger active surface area. ${ }^{53-}$ 61 The active layer of titania is also nanostructured and the use of nanoparticles of rareearth metal oxide nanoparticles to dope, as opposed to microparticles, could be more effective in improving the photovoltaic performance of DSSCs. The mechanism by which the REO nanoparticles interact with titania nanoparticles can be a powerful means for further steps in the development of more stable and more efficient DSSCs.

\subsection{Perovskites}

Originally, perovskite is a mineral 'calcium titanate $\left(\mathrm{CaTiO}_{3}\right)$ ' named after a Russian mineralogist Lev Perovski. Now perovskites are defined as a class of compounds, represented as $\mathrm{ABX}_{3}$, having a structure similar to that of $\mathrm{CaTiO}_{3}$, where $\mathrm{A}$ and $\mathrm{B}$ are cations and $\mathrm{X}$ is an anion. The lattice arrangement of $\mathrm{A}, \mathrm{B}$ and $\mathrm{X}$ in a unit cell of an ideal perovskite is shown in Figure 1.10a. An ideal perovskite has a simple cubic crystal structure consisting of a corner-sharing $\left[\mathrm{BX}_{6}\right]$ octahedral network with a $\mathrm{B}-\mathrm{X}-\mathrm{B}$ bond angle of $180^{\circ}$ and $\mathrm{A}$ ions in the interstices (Figure 1.10b). During crystallization, these units add up in all three directions, resulting in a 3D structure, so that it is referred to as a 3D perovskite.
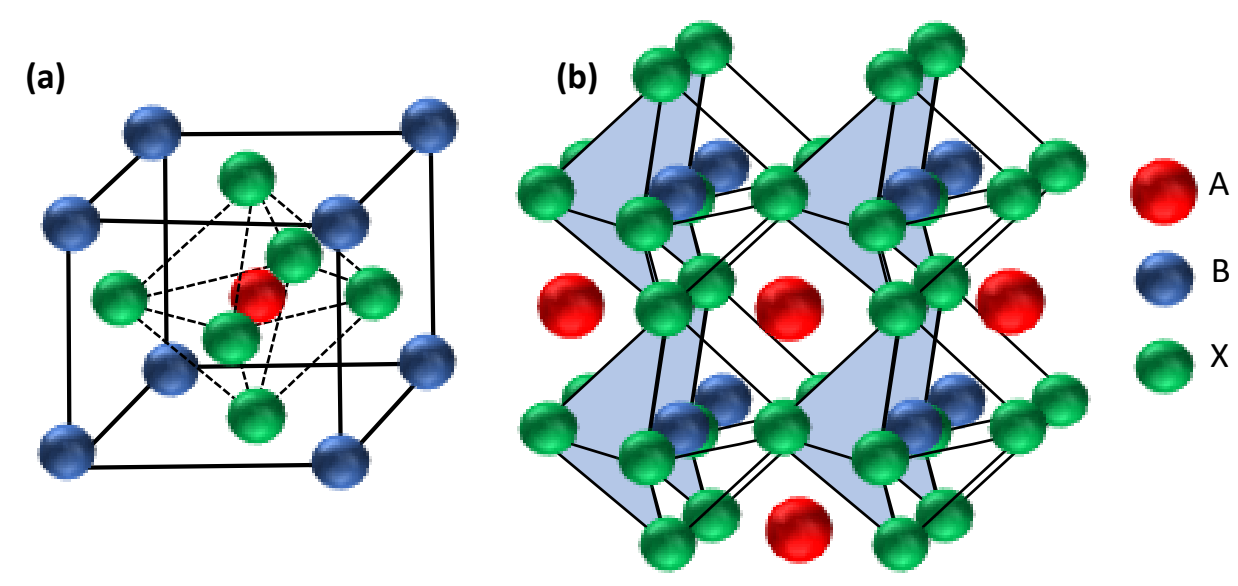

Figure 1.10. (a) Unit cell and (b) spatial distribution of A, B and X in a crystal lattice. 
To maintain an ideal perovskite structure, the selection of A, B and X should be made to meet the Goldschmidt's tolerance factor

$$
t=\frac{r_{A}+r_{X}}{\sqrt{2}\left(r_{B}+r_{X}\right)}
$$

where $\mathrm{r}$ is the radius of the ion mentioned in the subscript position. For most 3D perovskites $0.8 \leq t \leq 1$.

\subsubsection{Hybrid Perovskites}

In a perovskite if $\mathrm{A}$ is an organic cation such as methylammonium (MA), formamidinium (FA), or guanidinium (GA), and $\mathrm{B}$ is an inorganic cation such as $\mathrm{Pb}^{2+}$ or $\mathrm{Sn}^{2+}$, the perovskite is called an organic/inorganic perovskite or simply a hybrid perovskite (HP). The anions in HPs are usually halides. HPs have attracted intensive attention for optoelectronic applications for their various advantageous properties as described below:

(a) Long diffusion length. When solar light is incident on a semiconductor, electronhole pairs are generated. Diffusion length is the average length a photogenerated carrier (electron/hole) moves between the time of generation to the time of recombination. Long diffusion length is beneficial for the application in optoelectronic devices where recombination needs to be minimized. The diffusion length of a trihalide $\mathrm{HP}\left(\mathrm{MAPbI}_{3}\right)$ is in the range of 100-200 nm while the diffusion length of mixed halide $\mathrm{HP}\left(\mathrm{MAPbCl}_{\mathrm{x}} \mathrm{I}_{3-\mathrm{x}}\right)$ is longer than $1 \mu \mathrm{m} .{ }^{62}$

(b) High absorption coefficient. The absorption coefficient is a measure of how far into a material light of a particular wavelength can penetrate before it is absorbed. HPs have high absorption coefficient due to which they are good light-harvesting material for their application in optoelectronic devices. ${ }^{63}$ The absorption coefficient of these perovskites are on the order of $10^{5} \mathrm{~cm}^{-1},{ }^{64-67}$ which allows efficient light absorption in a film of thickness 300 500 $\mathrm{nm}$. The high absorption coefficient of HPs leads to increase in the quantum efficiency, and photocurrent in perovskites. 
(c) Low excitonic binding energy. An exciton is the bound state of an electron-hole pair, which are attracted to each other by an electrostatic interaction. Excitons are formed when photons with energy equal to or larger than the band gap energy are absorbed by a semiconductor. The minimum amount of energy required for the separation of these excitons is the excitonic binding energy. The materials used in optoelectronic devices should have low excitonic binding energy for their effective applications. HPs have low binding energy (37-75 meV) due to which the photogenerated electron-hole pairs are easily separated. ${ }^{68}$ The built-in potential can play a significant role in the separation of excitons. For the effective separation of excitons in a perovskite solar cell (discussed in a later section), electron transport and hole transport layers are used. The work function difference of these materials helps in the separation of excitons.

(d) Tunable bandgap. The bandgap is the energy difference between the conduction band and the valence band. It is the minimum amount of energy required by an electron to get excited from the valence band to the conduction band. For effective absorption of solar energy in the visible region $(3.1 \mathrm{eV}-1.7 \mathrm{eV})$ ), the bandgap energy should be $1.7 \mathrm{eV}$ or less. The bandgap of a HP depends on its composition. Usually halides and metal ions determine the bandgap. The commonly used $\mathrm{MAPbI}_{3} \mathrm{HP}$ has a bandgap of $1.6 \mathrm{eV}$ while the mixed halide HPs have a tunable bandgap. ${ }^{69}$ The bandgap of $\mathrm{MAPbBr}_{x} \mathrm{I}_{3-x}$ can be continuously tuned over the range of $1.6-2.3 \mathrm{eV}$ for $\mathrm{x}=0$ to $3 .{ }^{70}$ Similarly, the bandgap of $\mathrm{MAPbCl}_{x} \mathrm{I}_{3-\mathrm{x}}$ has been found between $1.6-2.9 \mathrm{eV}$ for $\mathrm{x}=0$ to $3 .^{71}$ This property of HPs makes them suitable for their applications in the various optoelectronic devices. ${ }^{68,72-76}$

(e) Photo/electroluminescence. Photoluminescence is a process in which the absorption of a photon by a molecule causes the excitation of one its electrons to a higher electronic state, and the electron returns to a lower energy level with the emission of radiation. HPs are found to possess photo/electroluminescence due to which they are popularly used in making multicolored light-emitting diodes. ${ }^{68}$

(f) Resistive switching. Resistive switching refers to tunable resistance states induced by an external electric field. HPs possess a resistive switching mechanism, due to which hysteretic behavior is observed in current-potential curves of perovskite solar cells 
(PSCs). Although the hysteresis is detrimental for PSCs, it is potentially useful for nonvolatile memory applications such as HP memristors. ${ }^{77-79}$

\subsubsection{Electric Polarization in Hybrid Perovskites}

Electric polarization is the process of development of an electrical double layer in a material. It can be understood with a parallel plate capacitor as shown in Figure 1.11. There is a dielectric material between the plates which gets polarized when an electrical field is applied. The polarized dipoles cause an induction of opposite charge on the plate. The polarization is then calculated using the induced electric field $\left(E_{\text {in }}\right)$ by

$$
\mathrm{P}=\varepsilon_{0} \varepsilon_{\mathrm{r}} \mathrm{E}_{\mathrm{in}}
$$

where $\varepsilon_{0}$ is the permittivity of the vacuum and $\varepsilon_{r}$ is the relative dielectric constant of the medium. The polarization can be ionic (displacement of oppositely charged ions by an electric field mostly found in ionic solids), electronic (displacement of electron clouds of an atom or valence electrons in covalent solids with respect to the positive ionic cores) or dipolar orientation of molecules with a permanent dipole moment. When the polarization is spontaneous and can be switched by applying a suitable electric field, it is called ferroelectric polarization.

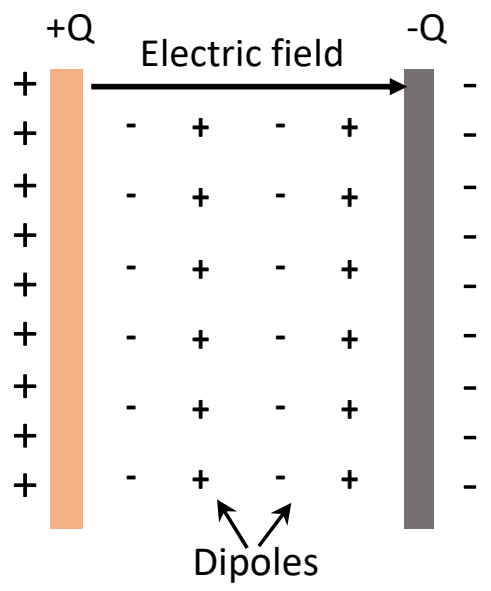

Figure 1.11. Parallel plate capacitor. When an electric field is applied, the dielectric gets polarized, which induces opposite charge on the plates. 
HPs have only methylammonium ions as the species with a permanent dipole of 2.3 D. The solution processed films of HPs have point defects which cause structural distortions. ${ }^{80-82}$ Also, there is vacancy mediated migration of ions in the crystal lattice. ${ }^{83}$ All these factors can cause the spontaneous and electrical field-induced polarization of the films of HPs. There is an ongoing debate on the question of whether hybrid perovskites are ferroelectric or not and how this might impact the optoelectronic devices. Hybrid perovskites show ferroelectric behavior at certain compositions and range of temperatures, which together govern the lattice structure. The ferroelectric polarization of hybrid perovskites is studied by a piezoresponse force microscopy (PFM) investigation and double wave method. ${ }^{84-87}$

\subsubsection{Perovskite Solar Cells}

A perovskite solar cell (PSC) uses a perovskite structured compound as the lightharvesting active layer. A hybrid organic-inorganic lead or tin-based material is commonly used in a PSC. Perovskite materials such as methylammonium lead halide and all inorganic cesium lead halide are cheap to produce and simple to manufacture.

\subsubsection{Working Principle of Perovskite Solar Cell}

One of the most important factors for evaluating the overall photovoltaic performance of a perovskite solar cell (PSC) is its architecture. Electron transport material (ETM) and hole transport material (HTM) are used along with the perovskite material. Depending on which transport material (ETM or HTM) is present on the exterior portion of the cell or where the incident light falls, the structures are classified as mesoscopic and planar. The mesoscopic layer consists of a mesoporous layer whereas the planar structure has all planar layers. These structures can be n-i-p, where the incident light passes to the perovskite layer through ETM, or p-i-n, where the incident light reaches the perovskite layer though HTM. All these structures are depicted in Figure 1.12 a-d. The first perovskite solar cell, where the light-harvesting dye of a DSSC was replaced with lead halide perovskite and liquid electrolyte with a solid-state HTM, had mesoporous n-i-p structure. It had a configuration 
of FTO anode/ETM/mesoporous oxide/perovskite/HTM/metal cathode. The planar structure is an evolution of the mesoscopic structure, where the perovskite-light harvesting layer is sandwiched between the ETM and HTM. We are using planar p-i-n structures for our solar cells discussed in Chapter VI.

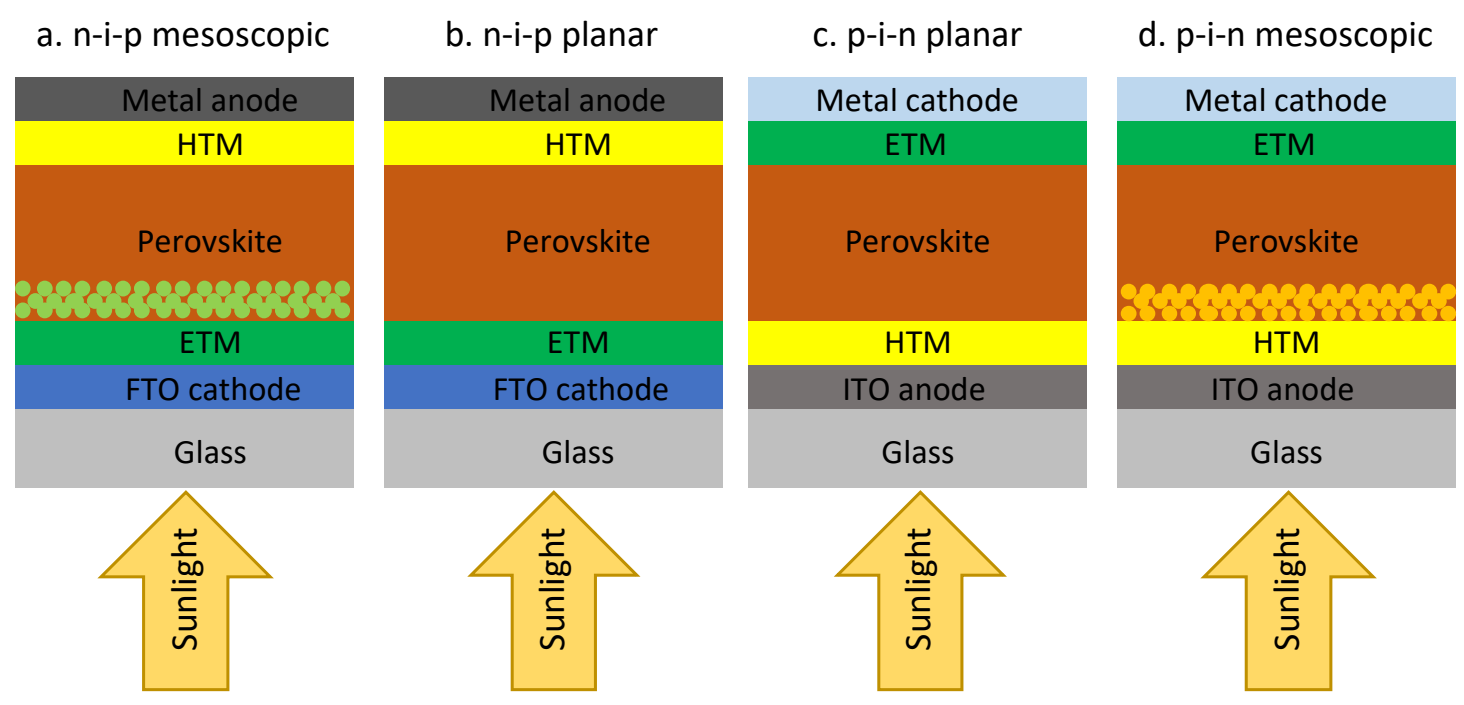

Figure 1.12. Architectures of different types of perovskite solar cells.

The overall working of a PSC is depicted with the help of band diagrams of the various components (Figure 1.13). It can be described in three steps: (i) absorption of sunlight to generate charge carriers, (ii) separation of charge carriers, and (iii) extraction of charges to generate electric current. When the solar radiation is incident on a PSC, the perovskite absorbs photons to generate electron-hole pairs, excitons. Excitons have some kind of binding energy because they are oppositely charged. This energy is in fact excitonic binding energy. The work function difference of the electron transport layer (ETL) and hole transport layer (HTL) provides the energy necessary for the dissociation of the excitons. The electron gets separated from the hole, getting injected to the ETL and then to the anode, while the hole gets injected to the HTL and then to the cathode. The potential difference between the cathode and the anode pushes the electrons to the external circuit to produce electric current. 


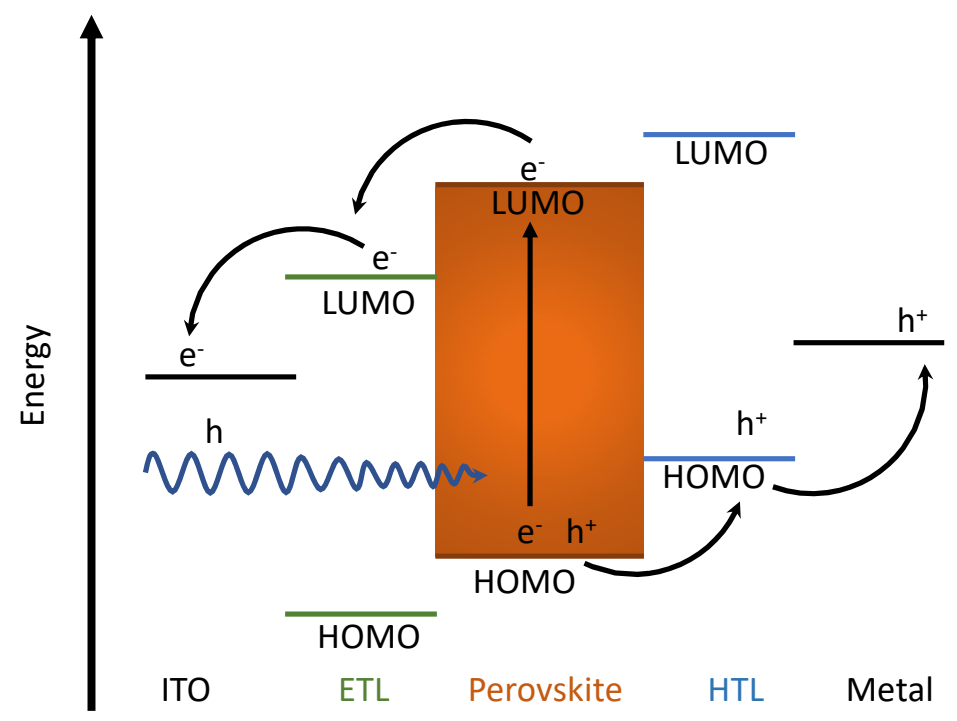

Figure 1.13. Working principle of a perovskite solar cell with band diagrams. These band diagrams represent a planar n-i-p perovskite solar cell.

\subsubsection{Issues with Perovskite Solar Cells}

The main issues with perovskite solar cells are the insufficient stability, the toxicity of lead, and the hysteresis often observed in the perovskite current-voltage (I-V) characteristics. ${ }^{88-89}$ Instead of $\mathrm{Pb}$ metal, other environmentally friendly metals such as $\mathrm{Sn}$ are used. Putting aside the toxic nature of lead, the structural, thermal, chemical and photostability are the chief challenges in the large scale implementation of perovskite solar cells (PSCs). ${ }^{90-91}$ Progress has been made for improvement in the stability through the management of the organic cations, inorganic cations and halide ions used in the material. ${ }^{71}$, 73, 90, 92 Reports reveal that the possible mechanisms for the hysteretic phenomenon observed in perovskite solar cells are trapping of positive and negative carriers, and ion migration. ${ }^{93-97}$ These explanations all involve a bias dependent change in the electric polarization of the perovskite that is thought to modify the transport properties. This also implies that an internal electric field exists in the film, even at zero bias, and additional bias can alter the field further. Recent experiments have measured an internal potential drop in hybrid perovskites using Kelvin probe force microscopy ${ }^{98}$ and capacitance measurements, ${ }^{99}$ however, the measurements were specific to a particular sample and the 
relationships between the built-in potential and the applied external field, material quality and photocurrent efficiency were not explored.

\subsubsection{Two-Dimensional Hybrid Perovskites}

One of the solutions proposed to improve the stability of hybrid perovskites is in the form of layered perovskites. ${ }^{100-101}$ These layered perovskites in fact are the twodimensional derivatives of three-dimensional perovskites and are formed when 3D frameworks are sliced into well-defined 2D slabs. The 2D perovskites have the general formula of $\left(\mathrm{RNH}_{3}\right)_{2}(\mathrm{~A})_{\mathrm{n}-1} \mathrm{M}_{\mathrm{n}} \mathrm{X}_{3 \mathrm{n}+1}$ where $\mathrm{RNH}_{3}$ is a large aliphatic or aromatic alkylammonium cation acting as a spacer between the perovskite layers, A and $\mathrm{M}$ are cations and $\mathrm{X}$ anions which form the perovskite framework, and $\mathrm{n}$ represents the number of corner-sharing $\left[\mathrm{MX}_{6}\right]^{4-}$ octahedral layers sandwiched between the interdigitating bilayers of spacer ions. ${ }^{102}$ Commonly used $\mathrm{RNH}_{3}, \mathrm{~A}, \mathrm{~B}$, and $\mathrm{X}$ ions are given in Table 1.1. As $n$ tends to $\infty$, the structure becomes a three-dimensionally bonded perovskite crystal. The spatial distribution of n-butylammonium, methylammonium, lead and iodide ions in the different types of $2 \mathrm{D}$ hybrid perovskites $\left[\left(\mathrm{CH}_{3}\left(\mathrm{CH}_{2}\right)_{3} \mathrm{NH}_{3}\right)_{2}\left(\mathrm{CH}_{3} \mathrm{NH}_{2}\right)_{\mathrm{n}-1} \mathrm{~Pb}_{\mathrm{n}} \mathrm{I}_{3 \mathrm{n}+1}\right]$ are shown in Figure 1.14. These perovskites normally have a tetrahedral or orthorhombic structure and are more flexible and deformable. ${ }^{103-104}$ 2D hybrid perovskites have greater stability in ambient conditions, and are exciting potential candidates for the applications in optoelectronic devices.

Table 1.1. Different ions used in layered hybrid perovskites

\begin{tabular}{|l|l|l|l|}
\hline Spacer cation & Organic cation & Metal cation & Inorganic anion \\
\hline n-Butylammonium & Methylammonium & $\mathrm{Pb}^{2+}$ & $\mathrm{Cl}^{-}$ \\
n-Propylammonium & Formamidinium & $\mathrm{Sn}^{2+}$ & $\mathrm{Br}^{-}$ \\
n-Octylammonium & Guanidinium & & $\mathrm{I}^{-}$ \\
n-Decaoctylammonium & & & \\
Phenyl ethylammonium & & & \\
\hline
\end{tabular}




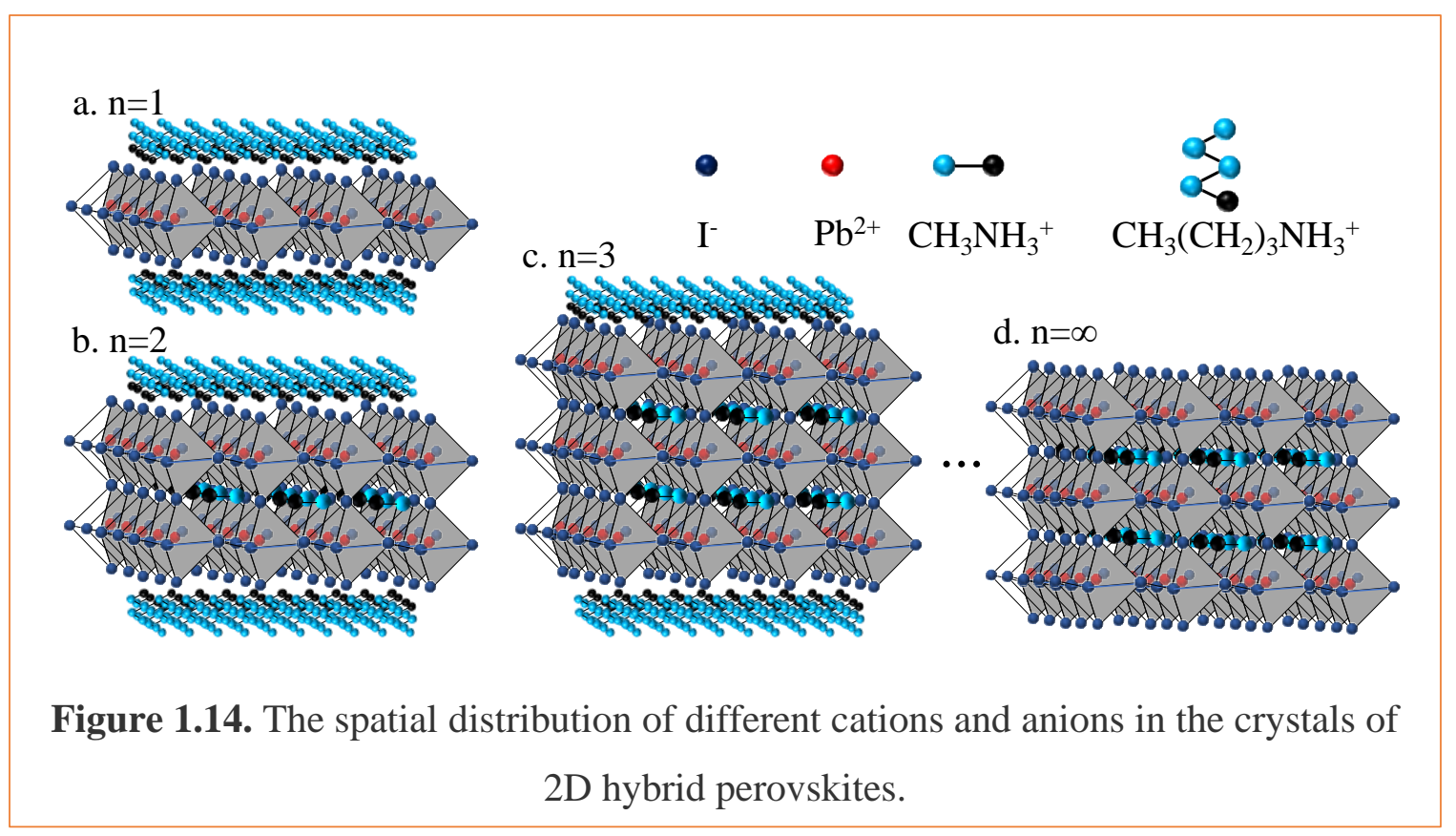

\subsubsection{Motivation for the Study of Perovskites}

The built-in potential developed in a hybrid perovskite film can help in the separation of photogenerated electron-hole pairs. The measurement of this intrinsic electric field is very important, and to the best of our knowledge, there is no technique that can directly measure this built-in potential in nano or micro domains. We developed a novel technique, the AC photocurrent measurement to measure the polarization of HP films. Using this technique, we did an intensive research on the grain size dependence of the polarization and its impact on the charge transport properties.

The stability of perovskite solar cells under ambient conditions is a very challenging issue. These solar cells are often fabricated and sealed in an environment of inert gases like Ar and $\mathrm{N}_{2}$. It is reported that light-soaking causes enhancement in the photoconversion efficiency of perovskite solar cells without considering the effect of background environment. Reports show different performance of perovskite solar cells with identical configuration when the measurements were carried out in different environments. This 
encouraged us to think about the effect of Ar gas and other gases on the electric polarization and photovoltaic performance of hybrid perovskite solar cells.

2D hybrid perovskites are emerging perovskites with improved stability and highly tunable bandgaps. The exploration of their optoelectronic properties can give a suitable direction for their applications in different optoelectronic devices.

\subsection{Outline of the Dissertation}

Chapter II of this dissertation presents the common experimental methods related to DSSCs and studies with 2D and 3D perovskites. It describes the fabrication of highly reproducible DSSCs, and the measurements done with the complete DSSCs, and titania (doped and undoped) films. In addition, this chapter discusses the synthesis of precursor materials for mixed halide hybrid perovskites, fabrication of good quality perovskite solar cells, and a novel technique, the AC photocurrent (ACP) method for photoelectrical characterization of hybrid perovskites. Chapter III describes the effect of rare-earth metal oxide (REO) nanoparticles on the photovoltaic performance of DSSCs. The various factors which contribute to the photovoltaic enhancement of DSSCs by the effect of REO nanoparticles doped into titania are well illustrated in this chapter. Chapter IV focuses on the studies of films made up of undoped titania and titania doped with REO nanoparticles by using electrical and electrochemical approaches. It also discusses the charge transport mechanism in nanostructured titanium dioxide (NTD) when doped with REO nanoparticles with the help of cyclic voltammetry, chronoamperometry, and electrochemical impedance spectroscopy. Chapter V describes the application of the AC photocurrent method to characterize the electric polarization of mixed halide hybrid perovskite films. The measurement of band-edge, built-in potential resulting from the polarization of the film, grain size dependence of the electric polarization, and carrier relaxation after poling are thoroughly described in this chapter. Chapter VI is about the effect of background environment on the electric polarization of mixed halide HPs. This chapter describes that the film of mixed halide HP adsorbs Ar gas in the presence of sunlight and enhances the 
electric polarization. The enhanced polarization leads to an increase in the photocurrent, which could be related to the improved photovoltaic performance. The test of this observation in an actual perovskite solar cell reveals that the combined effect of the Ar gas and sunlight in fact is responsible for the photovoltaic improvement. Chapter VII is related to the synthesis of crystals of layered hybrid perovskites and their characterizing with AC photocurrent and other techniques. Finally, the chief conclusions and directions for future studies are presented in Chapter VIII. 


\section{CHAPTER II}

\section{EXPERIMENTAL SECTION}

\subsection{Substrates}

Fluorine-doped tin oxide (FTO)-coated glass substrates were used for the fabrication of all DSSCs, electrochemical measurements with doped and undoped titania, and scanning electron microscopy (SEM) images. Indium tin oxide (ITO)-coated glass substrates were used for the fabrication of perovskite solar cells (PSCs). Glass substrates were used for all other experiments related to DSSCs, characterization of materials and experiments with perovskite materials.

\subsection{Chemicals and Materials}

Two types of Titania paste (18 NR-T and WER2-O), low temperature thermoplastic sealant $(30 \mu \mathrm{m})$, and a 43T mesh screen printer optimized for DSSCs were purchased from Dyesol, Australia. Anhydrous ethanol, acetone and ACS grade acetonitrile were obtained from VWR Internationals, USA. FTO glass (TEC 7) sheets of $6-8 \Omega / \mathrm{cm}^{2}$ were purchased from MTI Corporation, USA. ITO coated glass sheets (ITO-P001) of $<10 \Omega / \mathrm{cm}^{2}$ were bought from Zhuhai Kaivo Optoelectronic Technology Co., Ltd., China. 1,3dimethylimidazolium iodide, and $\mathrm{TiCl}_{4}$ were purchased from TCI Chemicals, USA. Cerasolzer was purchased from MBR electronics, Switzerland and T/SP platisol from Solaronix, Switzerland. $\mathrm{Nd}_{2} \mathrm{O}_{3}$ and $\mathrm{Er}_{2} \mathrm{O}_{3}$ nano powders each less than $100 \mathrm{~nm}$ in diameter, isopropanol, tert-butyl alcohol, C101 dye, iodine crystals, lithium iodide, lead oxide, lead 
iodide, $57 \% \mathrm{w} / \mathrm{w}$ aqueous hydroiodic acid, $40 \% \mathrm{w} / \mathrm{w}$ aqueous methylamine, $32 \% \mathrm{w} / \mathrm{w}$ aqueous hydrochloric acid, zinc nano powder, n-butylamine, N,N-dimethylformamide, valeronitrile, guanidinium thiocyanate, magnetic stir bars, methylammonium chloride were purchased from Sigma Aldrich, USA. $10 \mathrm{MHz}$ Au-coated quartz crystals were bought from Gamry Instruments, USA. Nanopure water of resistivity $18 \mathrm{M} \Omega-\mathrm{cm}$ was used for all cleaning procedures and solution preparations.

\subsection{Preparation of Reagents}

\subsubsection{Preparation of $\mathrm{TiCl}_{4}$ solution}

The aqueous solution of $\mathrm{TiCl}_{4}$ was prepared in nanopure water. First the water was cooled to a temperature below $5{ }^{0} \mathrm{C}$ and required volume of $\mathrm{TiCl}_{4}$ was added to it dropwise in a fume hood to make the final concentration of $40 \mathrm{mM}$. The solution appeared cloudy in the beginning, but it became clear after swirling the solution for some time. This solution was used for the deposition of hole blocking layer of titania nanoparticles (pre-treatment), and interconnecting titania particles of mesoporous film (post-treatment) as described in the section of fabrication of photoanodes of DSSCs.

\subsubsection{Preparation of Z960 Electrolyte}

The electrolyte containing redox couple iodide/triodide was prepared in a mixture of acetonitrile and valeronitrile. This electrolyte was used in all the DSSCs described later in this dissertation. The composition of the electrolyte was $1.0 \mathrm{M}$ 1,3-dimethylimidazolium iodide, $50 \mathrm{mM} \mathrm{LiI}, 30 \mathrm{mM} \mathrm{I}_{2}, 0.5 \mathrm{M}$ tert-butylpyridine and $0.1 \mathrm{M}$ guanidinium thiocyanate in a 17:3 mixture of acetonitrile and valeronitrile by volume.

\subsubsection{Preparation of C101 Dye Solution}

C101 dye was weighed and dissolved in a 1:1 mixture by volume of acetonitrile and tert-butyl alcohol to make the final concentration of $0.3 \mathrm{M}$. The dye solution was stirred 
magnetically for $30 \mathrm{~min}$ and stored in dark. This dye solution was used for the photosensitization of photoanodes of all DSSCs and dye-loading experiment.

\subsection{Cleaning Substrates}

Each type of substrate was cleaned using the following steps:

(i) Sonication with Alconox detergent solution in nanopure water for 15 minutes and rinsing with nanopure water.

(ii) Sonication in acetone for 10 minutes and rinsing with acetone.

(iii) Sonication in ethanol for 10 minutes and rinsing with ethanol.

(iv) Sonication in isopropanol for 10 minutes, rinsing with isopropanol and drying in $\mathrm{N}_{2}$ gas.

(v) Cleaning with UV/ozone treatment using a UVO-CLEANER Model No. 42 (Jelight Company, Inc.) for 15 minutes. This step destroys all organic impurities.

\subsection{Fabrication of Dye-Sensitized Solar Cells}

The fabrication method and components used in DSSCs, such as electrolyte, dye, titania nanoparticle diameter, titania film thickness, space between counter electrode and photoanode, and external contacts, can greatly influence the PEC parameters. This section describes the methods used to prepare DSSCs described later in this dissertation. It also describes the different measurements adopted to characterize the observed photovoltaic performance.

\subsubsection{Preparation of Titania Paste for Dye-Sensitized Solar Cell}

Dyesol 18 NR-T titania paste was used without any further modification for all undoped/traditional samples. The photoanodes made using this paste directly were named as 'Traditional' or 'Undoped'. $\mathrm{Nd}_{2} \mathrm{O}_{3}$ and $\mathrm{Er}_{2} \mathrm{O}_{3}$ oxide nanoparticles of less than $100 \mathrm{~nm}$ in diameter were doped into DSL 18 NR-T paste by mixing in the solid state. Briefly, about $2 \mathrm{~g}$ of DSL 18 NR-T paste was placed in a wide mouth short jar and the required amount of REO NPs was then added to it. The mixture was stirred with a glass pipette tip for 20 
minutes and left undisturbed for 2 hours with the cap closed, after which the paste was again stirred with a glass pipette tip for 20 minutes to make the distribution of REO NPs as homogeneous as possible. The photoanodes made from this paste were named as 'NdNPs-Solid' and 'ErNPs-Solid' to indicate the $\mathrm{Nd}_{2} \mathrm{O}_{3}$-doped and $\mathrm{Er}_{2} \mathrm{O}_{3}$-doped titania films, respectively. 'Solid' indicates that the REO NPs were added to the titania paste in the solid state.

\subsubsection{Fabrication of Photoanodes}

(a) Pre-TiCl4 treatment: The pre-cleaned slides were dipped in a $40 \mathrm{mM}$ aqueous solution of $\mathrm{TiCl}_{4}$ at $70{ }^{\circ} \mathrm{C}$ for 30 minutes followed by rinsing with nanopure water and then ethanol. The slides were then dried with a stream of nitrogen gas.

(b) Deposition of active layer: An approximately $7 \mu \mathrm{m}$ thick film of each undoped or doped titania paste was screen-printed onto the FTO using a Dyesol 43T Screen Printer. The film was placed together with a small piece of paper soaked in isopropanol and covered with a petri dish for 1 minute, where the isopropanol vapors aid in levelling the film. The film was then heated on a hot plate at $125{ }^{\circ} \mathrm{C}$ for 6 minutes to dry the film. Finally, a 4-5 $\mu \mathrm{m}$ thick scattering layer of 150-250 nm titania particles (DSL WER2-O) was screenprinted onto the film. For the best performing undoped/doped cells, undoped/doped paste was screen-printed twice. After each screen-printing, the film was placed together with a small piece of paper soaked in isopropanol and covered with a petri dish for 1 minute for levelling the film and was heated on a hot plate at $125{ }^{\circ} \mathrm{C}$ for 6 minutes to dry the film. This gave an average thickness of $11.3 \pm 0.5 \mu \mathrm{m}(\mathrm{n}=8)$. Finally, a $4-5 \mu \mathrm{m}$ thick scattering layer was screen-printed. The film was again heated at $125{ }^{\circ} \mathrm{C}$ for 15 minutes. The slides were then heated in a Barnstead Thermolyne 1300 furnace from room temperature to $300{ }^{\circ} \mathrm{C}$ using a temperature ramp of $10{ }^{\circ} \mathrm{C} / \mathrm{min}$ and held there for 15 minutes. They were then ramped from $300{ }^{\circ} \mathrm{C}$ up to $375^{\circ} \mathrm{C}$ and held there for 5 minutes. Finally, they were ramped from $375^{\circ} \mathrm{C}$ up to $500{ }^{\circ} \mathrm{C}$ and held there for 40 minutes. The temperature ramping 
program is shown in Figure 2.1. The slides were then gradually cooled to room temperature after the heating program. They were again treated with a $40 \mathrm{mM}$ aqueous solution of $\mathrm{TiCl}_{4}$ at $70{ }^{\circ} \mathrm{C}$ for 30 minutes, rinsed with nanopure water, and then with ethanol. The next step was heating in the furnace by ramping the temperature $10^{\circ} \mathrm{C} / \mathrm{min}$ up to $500{ }^{\circ} \mathrm{C}$ and holding at $500{ }^{\circ} \mathrm{C}$ for 30 minutes. The slides were gradually cooled to $80{ }^{\circ} \mathrm{C}$. These are the photoanodes used for making cells. Scheme 2.1 depicts the overall procedure for the fabrication of the photoanodes. For UV/VIS spectroscopic measurements and dye loading experiments, the photoanodes were made in the same way but without the scattering layer.

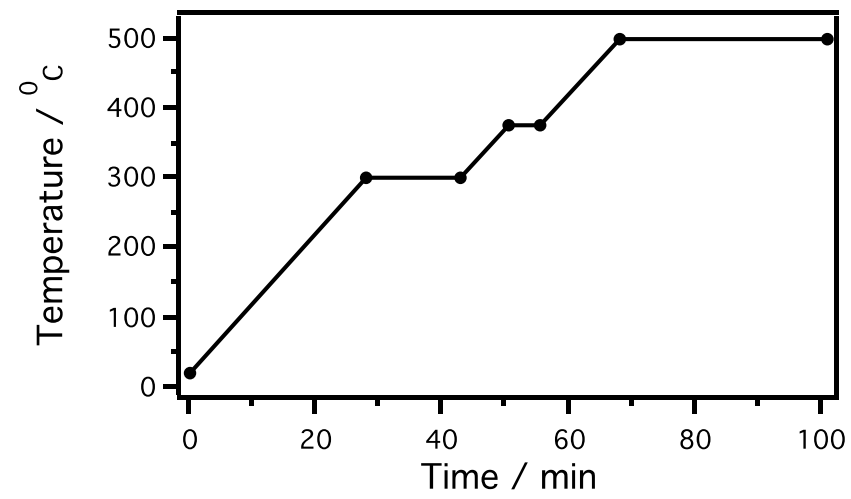

Figure 2.1. Temperature ramping program while sintering the films. 


\section{Scheme 2.1. Preparation of photoanodes for DSSCs.}

\begin{tabular}{|c|c|}
\hline 1. Dicing FTO slides into $2 \mathrm{~cm} \times 3 \mathrm{~cm}$ & 8. Heating on a hot plate at $125^{\circ} \mathrm{C}$ for $6 \mathrm{~min}$ \\
\hline $\begin{array}{l}\text { 2. Cleaning in detergent, acetone, ethanol, and } \\
\text { isopropanol for } 5 \text { min each }\end{array}$ & $\begin{array}{l}\text { 9. Heating inside an oven as described in } \\
\text { Figure } 2.1 \text {. followed by self-cooling to room } \\
\text { temperature }\end{array}$ \\
\hline 3. Cleaning with UV/ozone treatment & $\begin{array}{l}\text { 10. Post treatment with } 40 \mathrm{mM} \mathrm{TiCl}_{4} \\
\text { solution at } 70^{\circ} \mathrm{C} \text { followed by rinsing with } \\
\text { water and ethanol, and drying with } \mathrm{N}_{2} \text { gas }\end{array}$ \\
\hline $\begin{array}{l}\text { 4. Treatment with } 40 \mathrm{mM} \mathrm{TiCl}_{4} \text { solution at } 70{ }^{\circ} \mathrm{C} \\
\text { followed by rinsing with water and ethanol, and } \\
\text { drying with } \mathrm{N}_{2} \text { gas }\end{array}$ & $\begin{array}{l}\text { water and ethanol, and drying with } \mathrm{N}_{2} \text { gas } \\
\text { 11. Heating at } 450{ }^{\circ} \mathrm{C} \text { for } 30 \mathrm{~min} \text {, and } \\
\text { allowing to cool to } 80^{\circ} \mathrm{C}\end{array}$ \\
\hline $\begin{array}{l}\text { 5. Deposition of } 7 \mu \mathrm{m} \text { thick active layer of } 20 \\
\text { nm titania (doped/undoped) by screen-printing }\end{array}$ & $\begin{array}{l}\text { 12. Soaking the anodes at } 80{ }^{\circ} \mathrm{C} \text { in } 0.3 \mathrm{M} \\
\mathrm{C} 101 \text { dye for } 24 \mathrm{~h}\end{array}$ \\
\hline $\begin{array}{l}\text { 6. Exposure to isopropanol vapor for } 1 \mathrm{~min} \text { and } \\
\text { heating on a hot plate at } 125^{\circ} \mathrm{C} \text { for } 6 \mathrm{~min}\end{array}$ & $\begin{array}{l}\text { 13. Soaking in dye solvent for } 2 \mathrm{~h} \text { to desorb } \\
\text { multilayer dye attached to titania }\end{array}$ \\
\hline $\begin{array}{l}\text { 7. Deposition of scattering layer of } 200-400 \mathrm{~nm} \\
\text { titania by screen-printing }\end{array}$ & 14. Rinsing with ethanol and dry with $\mathrm{N}_{2}$ gas \\
\hline
\end{tabular}

Scheme 2.2. Fabrication of photoanodes for best performing DSSCs.

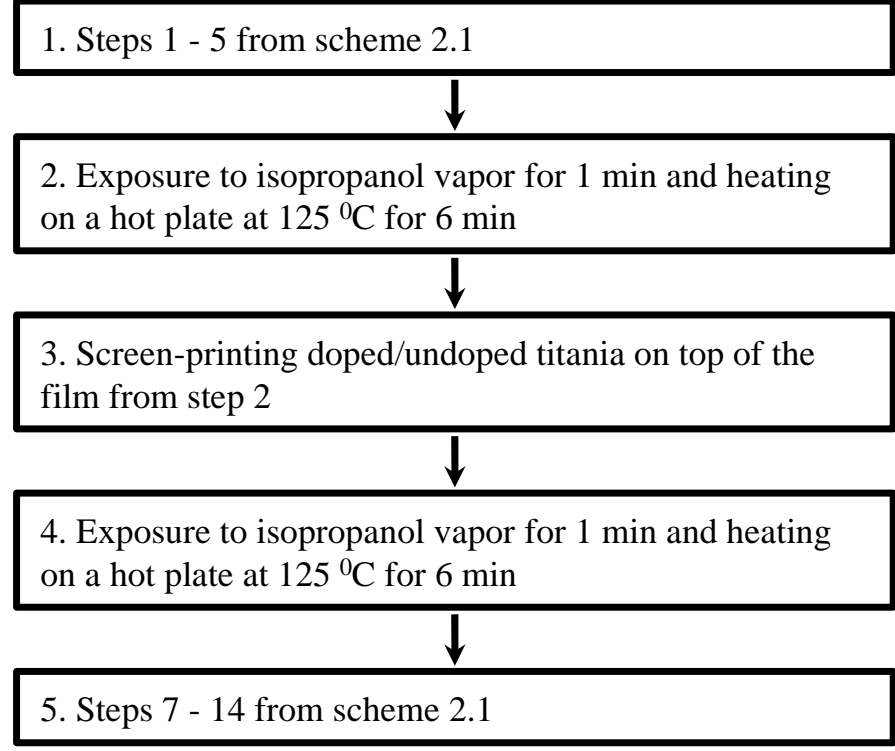




\subsubsection{Sensitization of Photoanodes with Dye}

The photoanodes at $80{ }^{\circ} \mathrm{C}$ in the oven were removed and immediately soaked in a 0.3 mM C101 dye in a 1:1 mixture by volume of acetonitrile and tert-butyl alcohol for 14 hours in the dark. They were removed from the solution and soaked in dye solvent for $2 \mathrm{~h}$ to desorb multilayer dye attached to titania. Finally, they were rinsed with ethanol and dried in a stream of $\mathrm{N}_{2}$ gas. These photoanodes were used to fabricate sandwich DSSCs. Figure 2.2a represents a photoanode sensitized with a dye.

\subsubsection{Fabrication of Counter Electrodes}

FTO slides were drilled with a $1 \mathrm{~mm}$ diamond drill bit (UKAM Industries, USA) at $5000 \mathrm{rpm}$ and cleaned using the procedure described above. Then, a $1 \mathrm{~cm} \times 1 \mathrm{~cm}$ area of T/SP platisol was screen-printed using a 43T Screen Printer in the center of the FTO near the hole. These slides were heated in a Barnstead Thermolyne 1300 furnace at $450{ }^{\circ} \mathrm{C}$ for 30 minutes and allowed to cool gradually to room temperature. The overall procedure involved in the fabrication of the counter electrode is shown in Scheme 2.3. Figure 2.2.b depicts a counter electrode.

(a)

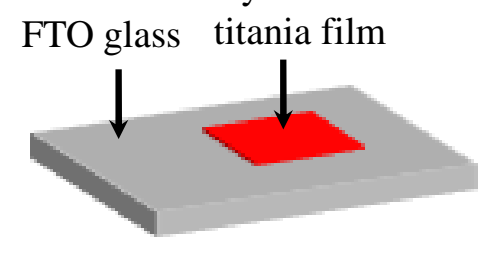

(b)

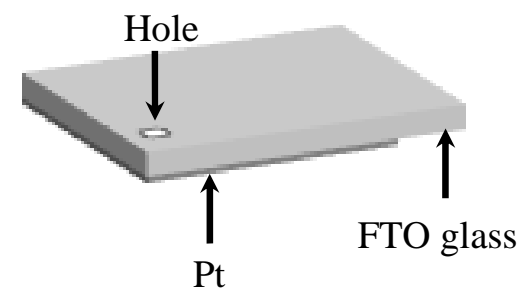

Figure 2.2. (a) Photoanode sensitized with a dye and (b) counter electrode used in DSSCs. 
Scheme 2.3. Fabrication of counter electrodes used in DSSCs.

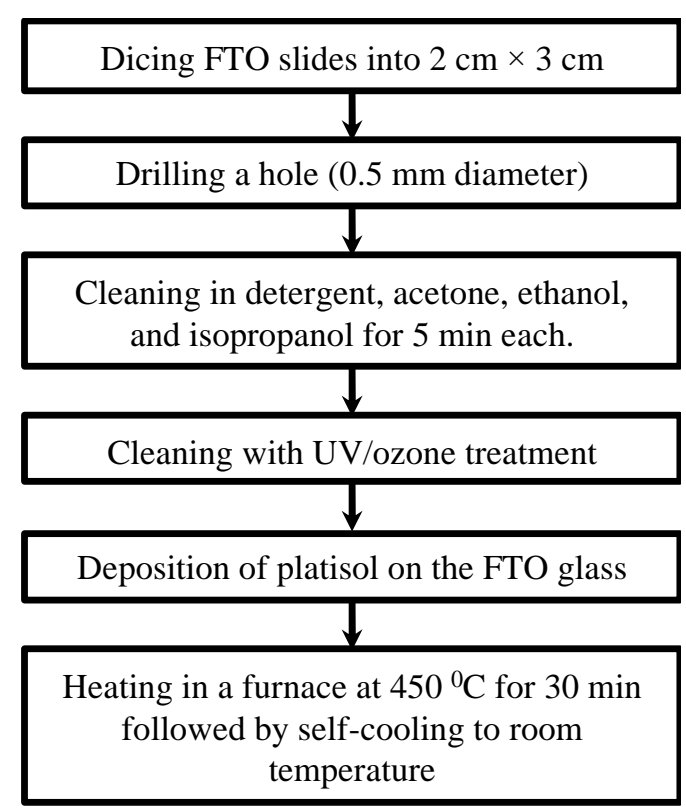

\subsubsection{Fabrication of Sandwich Dye-Sensitized Solar Cell}

The sandwich cells were fabricated by combining the photoanode sensitized with C101 dye as the working electrode with the platinum-coated FTO glass as the counter electrode, following a standard procedure ${ }^{39,105}$ with some modification. Briefly, $30 \mu \mathrm{m}$ low temperature thermoplastic sealant was inserted between the working electrode and the counter electrode and the two were held tight in contact with each other. The assembly was heated at $100{ }^{\circ} \mathrm{C}$ for 45 seconds using a thermal press MPRESS912 (HeatPress, USA). This process is illustrated in Figure 2.3. The electrical contacts were made at the end of each of the working and the counter electrodes using Cerasolzer (MBR Electronics, Switzerland) and a soldering iron at $200{ }^{\circ} \mathrm{C}$. Z960 electrolyte was injected into the internal space of the cell through the drilled hole by a vacuum backfilling system. ${ }^{106} \mathrm{~A}$ drop of the electrolyte was placed on the drilled hole of the counter electrode of the cell kept in a small vacuum chamber (Figure 2.4a). The repeated evacuation of the air followed by exposure to ambient pressure pushed the electrolyte into the internal space of the cell (Figure 2.4b-c). To seal the hole, a small piece of thermoplastic sealant was placed on the hole, and a coverslip ( 0.1 $\mathrm{mm}$ thickness) was placed onto the sealant. The assembly was heated at $100{ }^{\circ} \mathrm{C}$ under 
pressure. Figure $2.4 \mathrm{~d}$ shows a DSSC with after heating the assembly. Finally, the cell was masked in order to expose a fixed area to light. The mask used for this purpose was made from a black tap by laser cutting. The complete device fabrication process is shown in Scheme 2.3. A complete DSSC is shown in Figure 2.4e. The masked area was measured to be $0.283 \pm 0.003 \mathrm{~cm}^{2}(\mathrm{n}=5)$ with a measurement uncertainty for the $\mathrm{XY}$ axis of $\pm 2.3 \mu \mathrm{m}$. This area was used for all the calculations of PEC parameters.
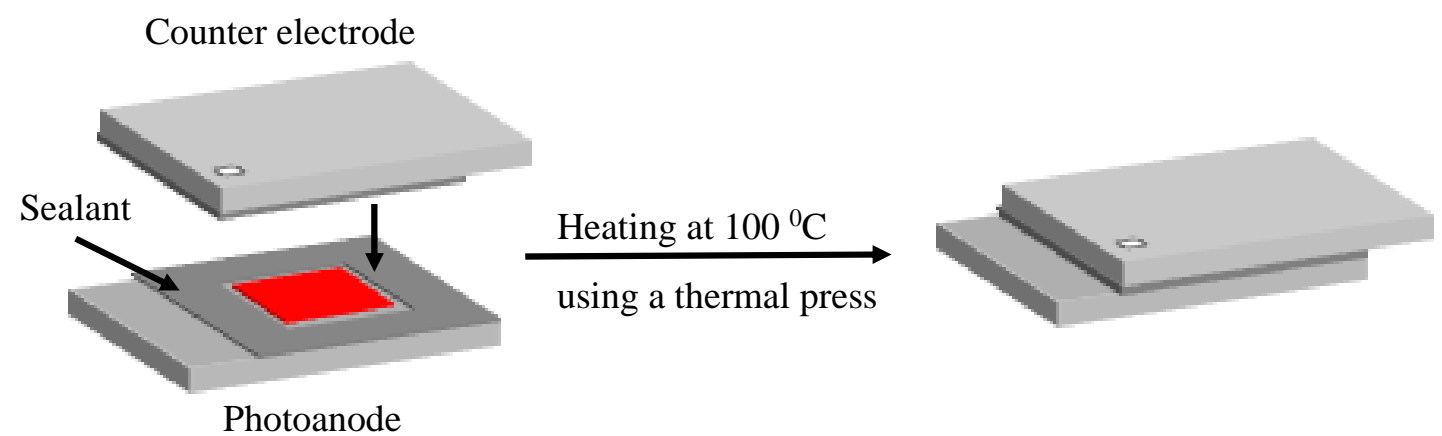

Figure 2.3. Assembling photoanode and counter electrode to make a sandwich DSSC. 
Scheme 2.4. Fabrication of a sandwich DSSC

Making a square window of sealant area a little larger than the area of photoanode

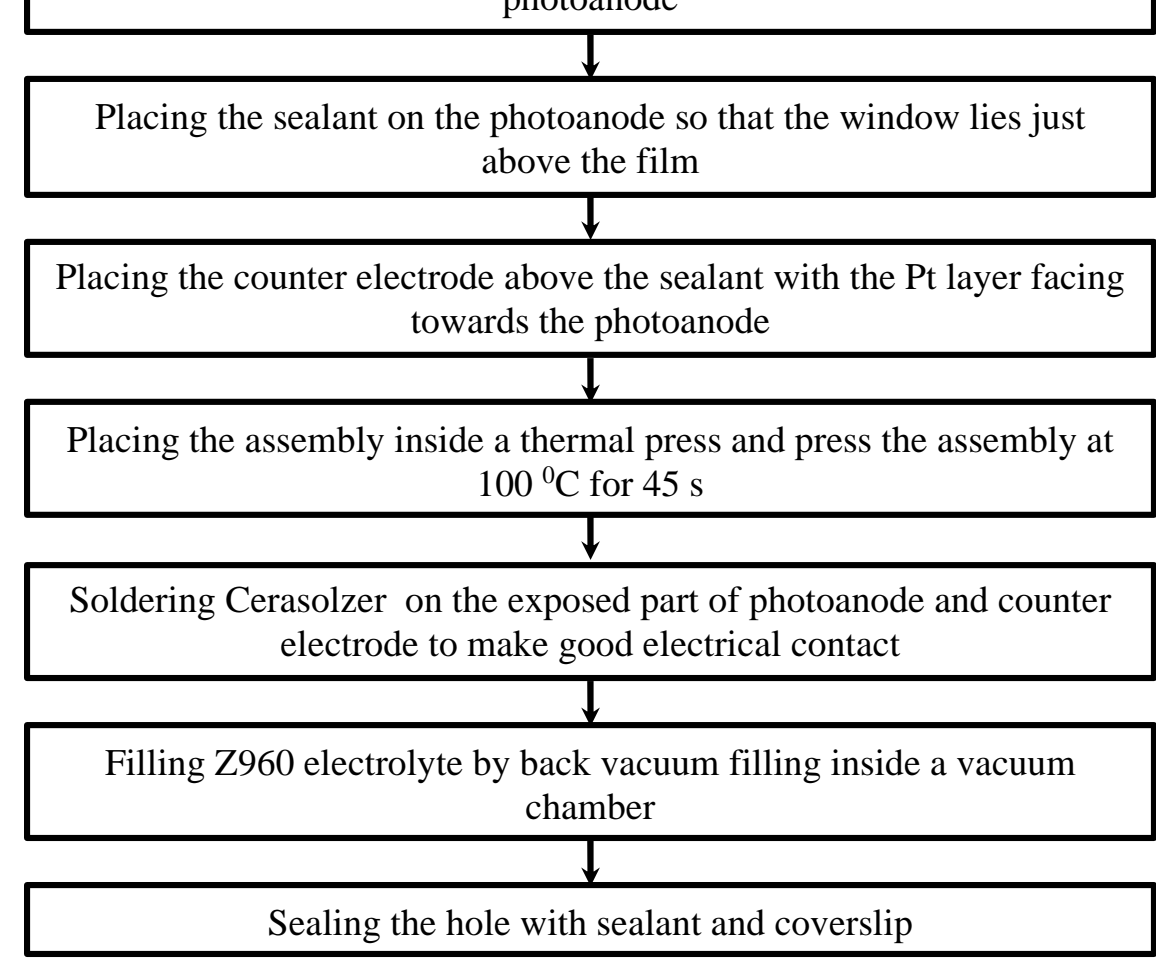

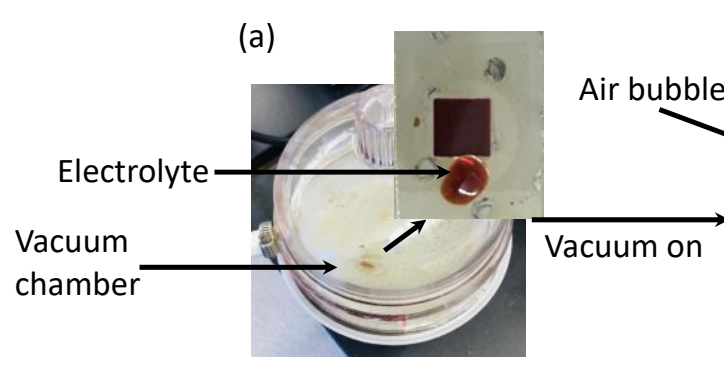

(d)

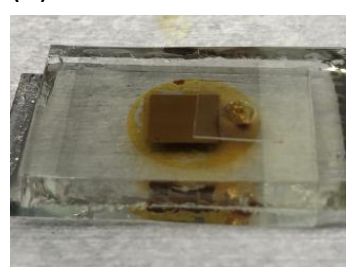

(b)

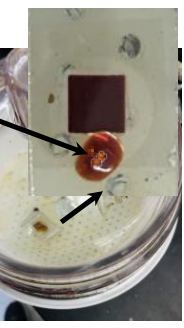

(e)

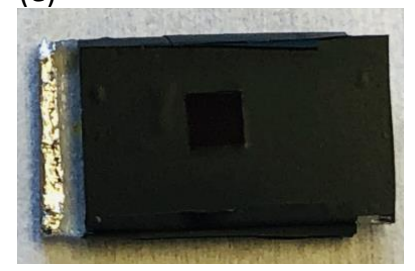

Figure 2.4. (a)-(c) Vacuum backfilling of electrolyte in a DSSC, (d) a DSSC with sealed hole, and (e) a complete DSSC with a mask. 


\subsection{Characterization of Photoanodes Used for Dye-Sensitized Solar Cells}

The optical images of the films were taken using a Zeiss Axio Imager version a $2 \mathrm{~m}$ Microscope. A Zeiss Supra 35 Scanning Electron Microscope was used to take SEM images. An Asylum MF3D atomic force microscope in AC mode was used to study the roughness of the surface of the films. Images each of $5 \mu \mathrm{m} \times 5 \mu \mathrm{m}$ size were taken at five different spots, and the average of five root-mean-square (RMS) roughness measurements was obtained. All the photoanodes used for the characterization had only an active layer of approximately $7 \mu \mathrm{m}$ thickness with no second layer and no scattering layer.

\subsection{I-V Measurements of Dye-Sensitized Solar Cells}

For the photovoltaic measurements, a cell was exposed to the light from Newport's LCS-100 solar simulator maintained at one sun using a calibrated silicon solar cell. For the calibration of solar simulator, the standard silicon solar cell was exposed to the light from the solar simulator, and the photocurrent was measured as a function of external potential. The distance of the standard silicon solar cell from the light source was adjusted to get the photocurrent certified for one sun power. Then a DSSC was placed exactly at the same position as the standard silicon solar cell and the photocurrent was measured as a function of external potential from $0.5 \mathrm{~V}$ to $-0.8 \mathrm{~V}$ using a Keithley 2401 Potentiostat. The experimental setup for the I-V measurement is shown in Figure 2.5.

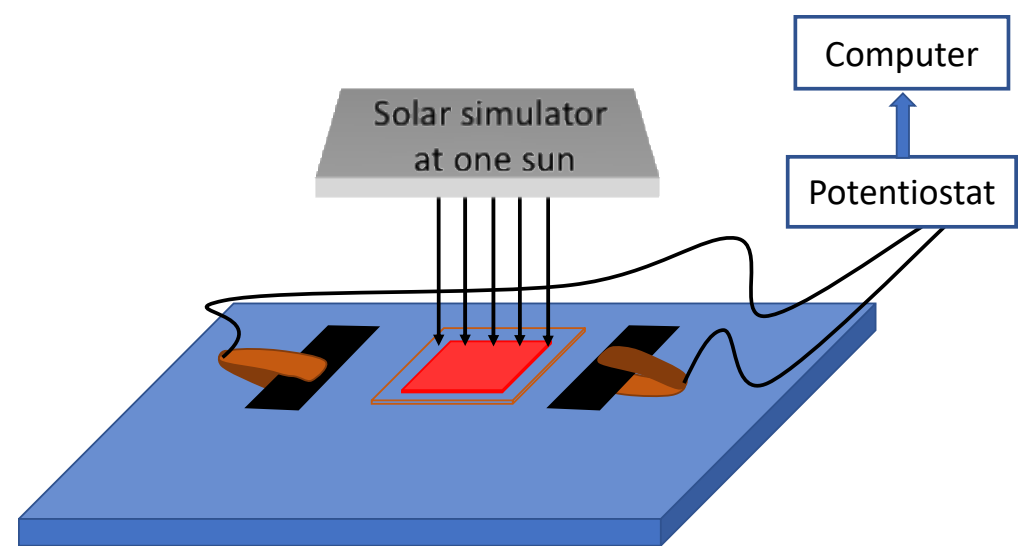

Figure 2.5. Illustration of I-V measurement of a DSSC. 


\subsection{Electrochemical Impedance Spectroscopy (EIS) Measurements of Dye-Sensitized Solar Cells}

All EIS measurements were conducted in the dark at $-680 \mathrm{mV}$ using a CHI 660E instrument by applying an $\mathrm{AC}$ signal of $10 \mathrm{mV}$ at frequencies ranging from $10 \mathrm{mHz}$ to 100 $\mathrm{kHz}$.

\subsection{BET Analysis of Doped and Undoped Titania}

For the measurement of the specific area of doped and undoped titania, about 100$200 \mathrm{mg}$ of each of doped and undoped titania was made by screen-printing and sintering as mentioned above. Each sample was kept in a clean and dry BET tube and degassed at $80{ }^{\circ} \mathrm{C}$ for 3 hours, and the weight of the dry sample was measured. It was then subjected to $\mathrm{N}_{2}$ gas adsorption and desorption using a Tristar III.

\subsection{Dye Loading in Doped and Undoped Titania}

For dye loading measurements, the photoanodes having a $7 \mu \mathrm{m}$ thick mesoporous film of titania doped with REO at different concentrations were immersed in a $0.3 \mathrm{mM}$ C101 dye solution for 14 hours in the dark. The photoanodes were soaked in dye solvent for $2 \mathrm{~h}$ to desorb multilayer dye attached to titania. They were then rinsed with ethanol and dried with $\mathrm{N}_{2}$ gas. The UV/VIS spectra of the dye loaded films were measured with a Perkin Elmer Lambda 950 UV/Vis Spectrometer. The optical absorbances at $550 \mathrm{~nm}$ were used to compare the amount of dye adsorbed on each type of film.

\subsection{Synthesis of Methylammonium Chloride}

Methylammonium chloride $(\mathrm{MACl})$ was synthesized by following the procedure described elsewhere. ${ }^{16}$ Briefly, $40 \%$ w/w aqueous $\mathrm{CH}_{3} \mathrm{NH}_{2}$ was neutralized by an equimolar quantity of $32 \% \mathrm{w} / \mathrm{w}$ aqueous $\mathrm{HCl}$ in an ice bath with constant stirring for $2 \mathrm{~h}$ 
as shown in Figure 2.6. After the reaction, white precipitate of $\mathrm{MACl}$ was recovered by rotary evaporation at $40{ }^{\circ} \mathrm{C}$. The precipitate was recrystallized in ethanol. The crystals were washed with cold diethyl ether three times and dried in vacuum at $40{ }^{\circ} \mathrm{C}$ for 2 hours. The details of the synthesis process are given in Scheme 2.5.

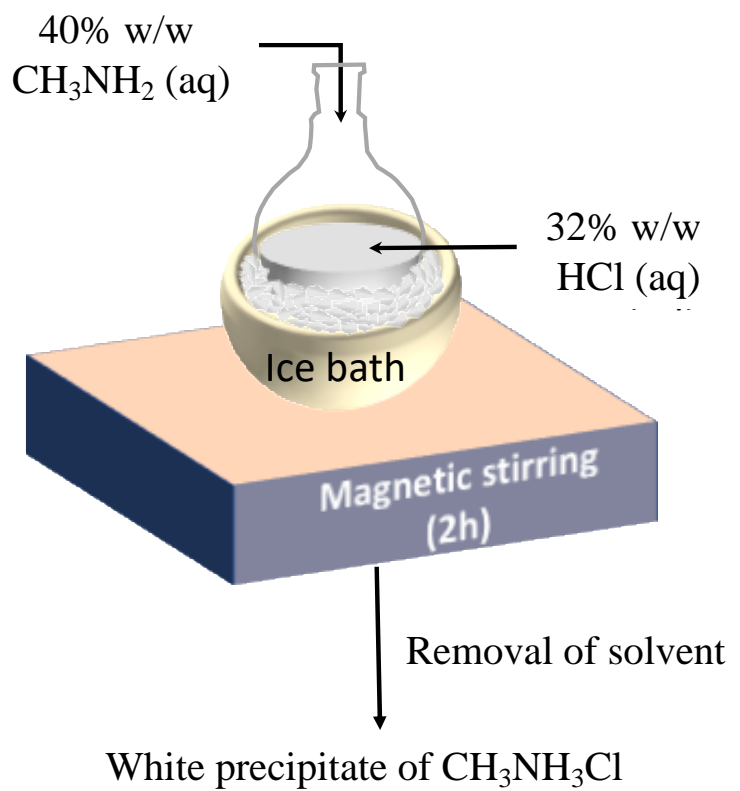

Figure 2.6. Synthesis of methylammonium chloride. 
Scheme 2.5. Synthesis of methylammonium chloride

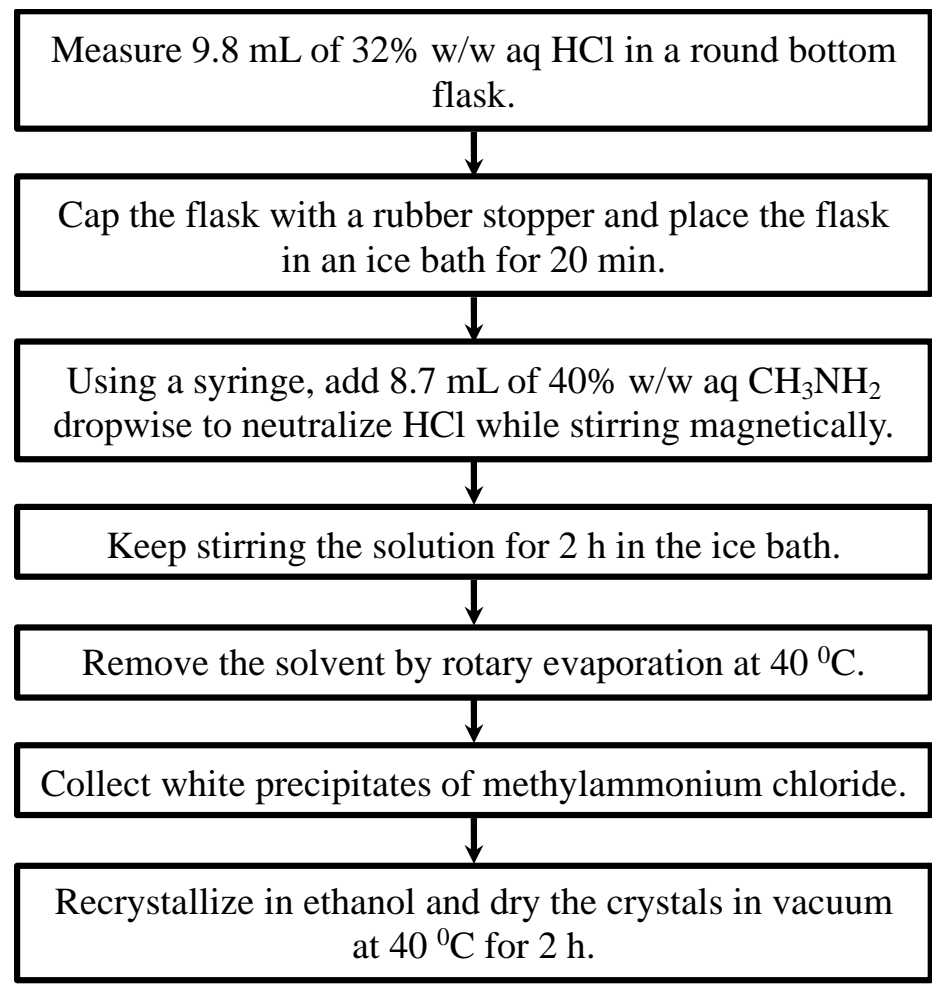

\subsection{Preparation of $\mathrm{CH}_{3} \mathrm{NH}_{3} \mathrm{PbCl}_{x} \mathrm{I}_{3-\mathrm{x}}$ Precursor Solution}

Unless otherwise mentioned, the precursor solution of lead-based methylammonium mixed halide containing chloride and iodide was prepared by mixing $\mathrm{PbI}_{2}$ and $\mathrm{MACl}(1.1: 1$ molar ratio) in anhydrous DMF to make a $0.2 \mathrm{M}$ solution with respect to $\mathrm{MACl}$. The mixture was stirred at $70{ }^{0} \mathrm{C}$ for 12 hours. This solution was used to cast the perovskite films used for electronic and electrochemical studies.

\subsection{Fabrication of Highly Granular Film}

Highly granular films for the various experiments with mixed halide hybrid perovskite containing chloride and iodide were prepared by using a hot-casting technique. ${ }^{16}$ Briefly, a pre-cleaned glass substrate was heated to $180{ }^{\circ} \mathrm{C}$ for 10 minutes and quickly transferred to the top of a spin-coater. $150 \mu \mathrm{L}$ of the precursor solution at $70{ }^{\circ} \mathrm{C}$ was then 
dropped to the center of the substrate within 5 seconds and spun immediately at $3000 \mathrm{rpm}$ for $30 \mathrm{~s}$ which gives large grain size films. Any delay in spin-coating results in smaller grain films and incomplete conversion of the precursors to the perovskite crystal structure. The hot-casting technique is illustrated in Figure 2.7.

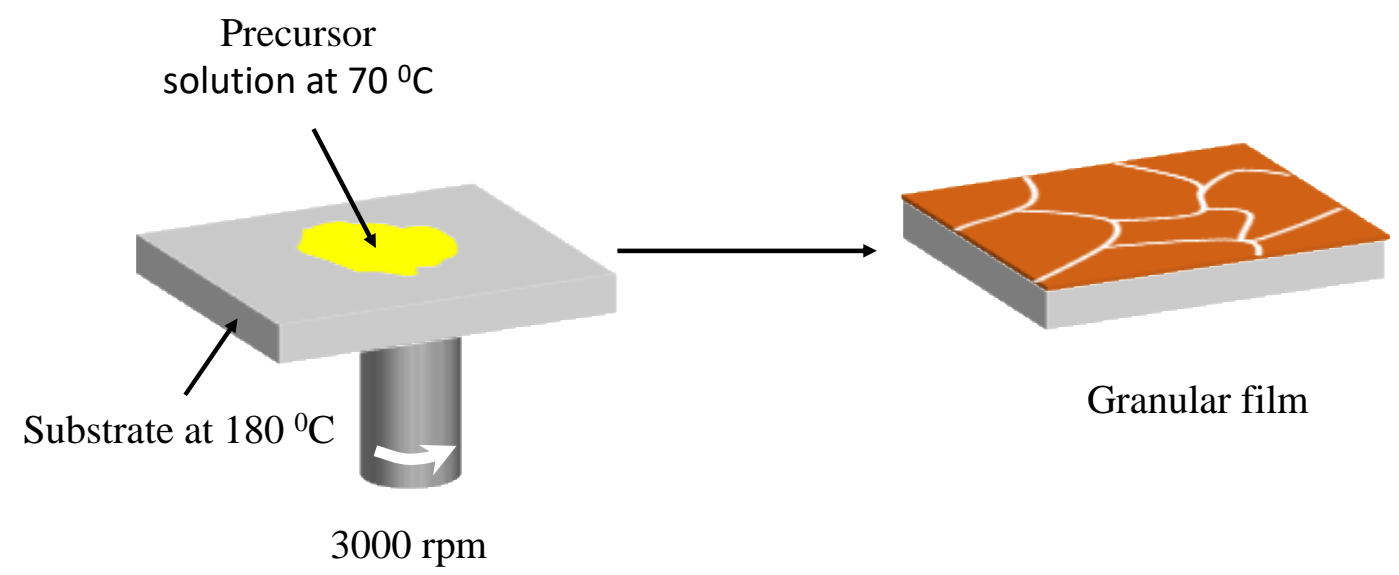

Figure 2.7. Hot casting method for making highly granular films.

\subsection{AC Photocurrent Measurement}

(a) Device fabrication. The precursor solution of methylammonium chloride (MACl) and $\mathrm{PbI}_{2}$ at $70{ }^{\circ} \mathrm{C}$ was spin-coated onto a cleaned glass slide using the hot-casting technique as mentioned above. The sample was quickly transferred to the electron beam evaporator to avoid any degradation due to air and moisture. $100 \mathrm{~nm}$ thick gold contacts were then evaporated onto the perovskite film. A shadow mask (Figure 2.8) was used to define an interdigitated contact pattern with 30-micron wide fingers separated by 40-micron gaps. Following the deposition of the Au contacts, the device was mounted in an optical cryostat and electrically connected with coppers wires using a conductive Ag paint. The cryostat was then pumped down to a vacuum of $10^{-7}$ torr before electrical measurements. The details of the device fabrication are given in Scheme 2.6. 
Scheme 2.6. Device fabrication for AC photocurrent measurements.

\begin{tabular}{|c|c|}
\hline $\begin{array}{l}\text { Weigh out } 14.6 \mathrm{mg} \text { of methylammonium } \\
\text { chloride and } 100 \mathrm{mg} \text { of lead iodide in a vial } \\
\text { and add } 1 \mathrm{~mL} \text { of anhydrous DMF }\end{array}$ & $\begin{array}{l}\text { Pump the cryostat to lower the pressure to } \\
\qquad 10^{-7} \text { torr. } \\
\qquad \uparrow\end{array}$ \\
\hline$\downarrow$ & Make electrical connections with $\mathrm{Cu}$ wires \\
\hline $\begin{array}{l}\text { Keep stirring the mixture at } 70{ }^{\circ} \mathrm{C} \text { for } 12 \mathrm{~h} \\
\text { to get a clear yellow solution. }\end{array}$ & $\frac{\text { using Ag paint. }}{\uparrow}$ \\
\hline$\frac{\downarrow}{\text { Clean microsconic olass slide in deteroent }}$ & $\begin{array}{l}\text { Take out the device from the electron beam } \\
\text { evaporator and mount in a cryostat. }\end{array}$ \\
\hline $\begin{array}{l}\text { Clean microscopic glass slide in detergent, } \\
\text { acetone, ethanol and isopropanol } 5 \mathrm{~min} \\
\text { each. }\end{array}$ & $\uparrow$ \\
\hline$\downarrow$ & $\uparrow$ \\
\hline $\begin{array}{c}\text { Treat the slide with UV/ozone for } 15 \mathrm{~min} \text {. } \\
\downarrow\end{array}$ & $\begin{array}{l}\text { Pump the evaporator to the pressure to } 10^{-7} \\
\text { torr before deposition. }\end{array}$ \\
\hline $\begin{array}{l}\text { Heat the slide at } 180{ }^{\circ} \mathrm{C} \text { for } 10 \mathrm{~min} \text { on a hot } \\
\text { plate and transfer to the top of spin-coater. }\end{array}$ & $\begin{array}{l}\uparrow \\
\text { Mount the assembly in the sample holder } \\
\text { and transfer to electron beam evaporator }\end{array}$ \\
\hline $\begin{array}{l}\text { Drop } 150 \mu \mathrm{L} \text { of the precursor solution on } \\
\text { the middle part of the slide and spin-coat at } \\
3000 \mathrm{rpm} \text { for } 30 \mathrm{~s} \text {. }\end{array}$ & $\begin{array}{c}\uparrow \\
\begin{array}{c}\text { Place a shadow mask on the top of the film } \\
\text { and tape it. }\end{array}\end{array}$ \\
\hline
\end{tabular}

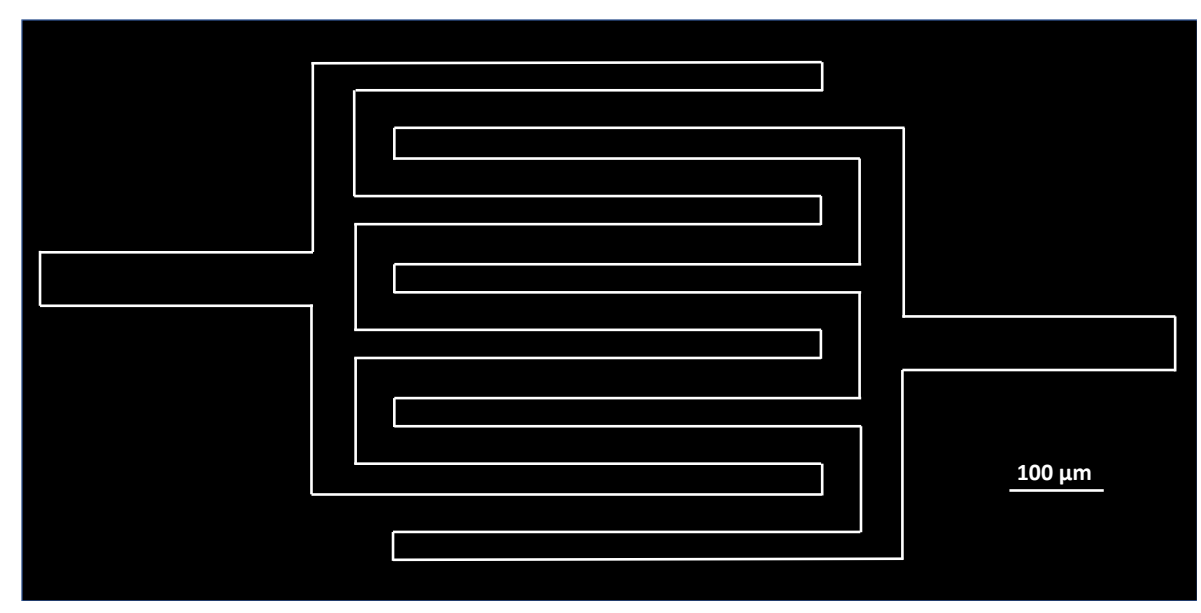

Figure 2.8. Shadow mask for the interdigitated contacts. 
(b) AC photocurrent spectrum measurement. Figure 2.9a shows the experimental setup for the AC photocurrent measurements. Briefly, the sample was exposed to the light from a tungsten bulb spectrally filtered using a monochromator. The light with an average power of $2.5 \mathrm{~mW} \mathrm{~cm}^{-2}$ was chopped at a frequency of $13 \mathrm{~Hz}$ using an optical chopper. The signal from the device was fed to a current amplifier, the output of which was fed to a lockin-amplifier. The output data from the lock-in amplifier were passed to the multimeter and to the computer. For obtaining the $\mathrm{AC}$ photocurrent spectrum, the photocurrent was measured as a function of the wavelength of light, selected by the monochromator.

(c) Built-in potential and phase measurements. Figure $2.9 \mathrm{~b}$ shows the experimental setup for built-in potential $\left(\mathrm{V}_{\mathrm{bi}}\right)$ and phase measurements. Here, an external potential is applied to the device with a source meter. For the determination of built-in potential, the AC photocurrent was measured as a function of external potential applied while the monochromatic, $13 \mathrm{~Hz}$-chopped light at maximum absorption wavelength (740 nm) was constantly striking the device. The potential range for a scan was determined by observing the output signal decreasing as the potential was scanned from $0 \mathrm{~V}$ towards the negative direction. A lock-in amplifier was kept in phase mode for the measurement of phase associated with the $\mathrm{AC}$ photocurrent. $\mathrm{V}_{\mathrm{bi}}$ is the potential where the $\mathrm{AC}$ photocurrent is zero (or at a minimum). 

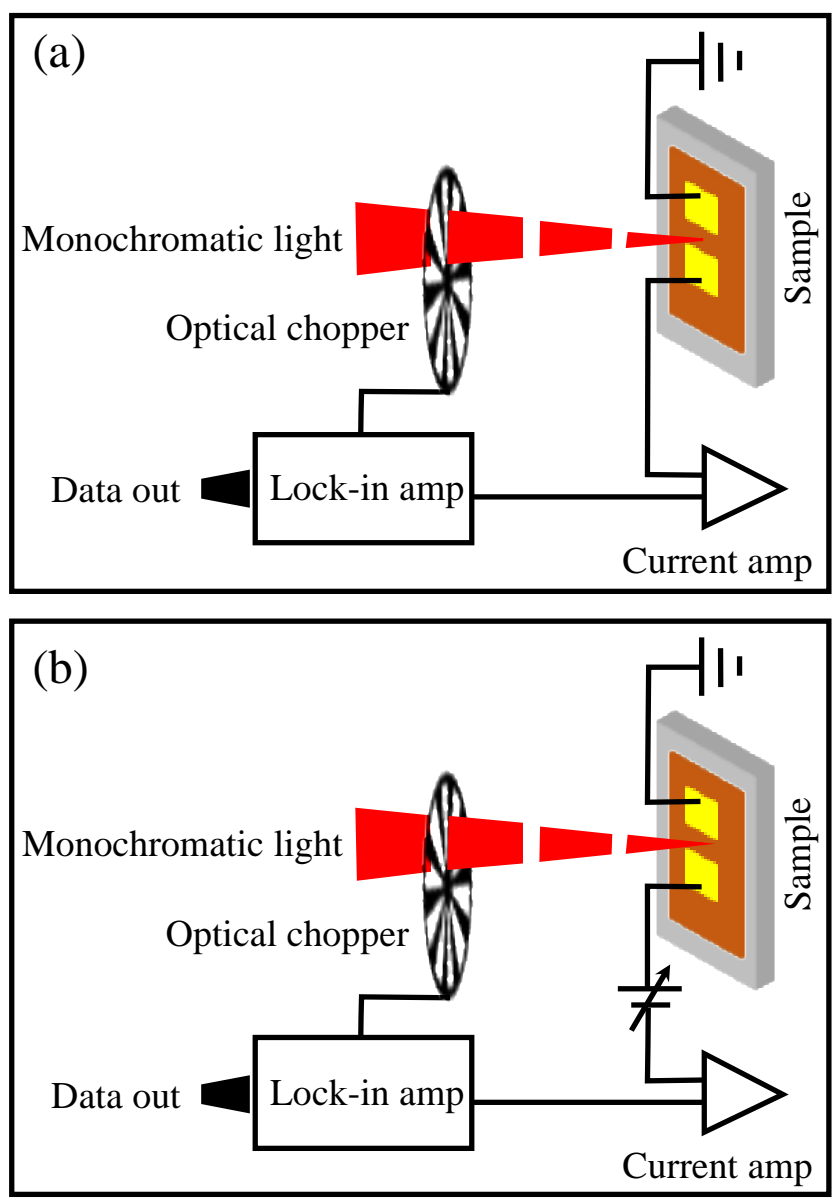

Figure 2.9. Experimental setup for the measurement of (a) AC photocurrent spectrum, and (b) built-in potential and phase.

(d) Electric polarization measurements. The devices used for the polarization measurements were the same as the other measurements. Polarization (P) was calculated from the built-in potential by

$$
P=\varepsilon_{0} \varepsilon_{r}\left(\frac{V_{b i}}{l}\right)
$$

where $l$ is the separation between the fingerlike contacts $(40 \mu \mathrm{m}), \varepsilon_{0}$ is the permittivity of vacuum, $\varepsilon_{r}$ is the relative dielectric constant of $\mathrm{MAPbI}_{\mathrm{x}} \mathrm{Cl}_{3-\mathrm{x}}$. The relative dielectric constant for MHHP was taken from literature for the frequency very close to zero. ${ }^{107}$ The intrinsic electric polarization was calculated from the built-in potential of pristine sample. 
For the electrical field induced polarization, a thin film of MHHP was poled at different potentials from 0 to $7.5 \mathrm{~V}$ to $-7.5 \mathrm{~V}$ to $7.5 \mathrm{~V}$ and the corresponding $\mathrm{V}_{\text {bi }}$ was measured at every $0.5 \mathrm{~V}$ increment. The device was poled for 5 minutes at each potential, and $\mathrm{V}_{\text {bi }}$ was measured within 40 seconds. The device was then kept at $0 \mathrm{~V}$ for 2 minutes to let the system relax close to the initial condition before every measurement.

For the built-in potential measurement of MHHP samples under different environments, the measurements were carried out as follows:

(i) The device was kept in vacuum for 30 minutes at a pressure of $10^{-7}$ torr and the built-in potential was measured every 3 minutes for about 20 minutes.

(ii) White light from a solar simulator was shone at the region of interdigitated finger contacts on the device and the built-in potential was measured every 3 minutes for about 20 minutes.

(iii) The vacuum was discontinued, and the cryostat was then filled with a continuous flow of Ar gas. The built-in potential was measured every 3 minutes for about 1 h.

(iv) The flow of Ar gas was then stopped and the Ar was pumped out to create a vacuum in the chamber of the cryostat. As the Ar gas was pumped out, the builtin potential was measured every 3 minutes for about $1 \mathrm{~h}$.

(v) The solar simulator was switched off. After 30 minutes, the vacuum was discontinued, and a constant flow of Ar was maintained.

(vi) Finally, the built-in potential was measured every 3 minutes for 20 minutes.

The details of built-in potential measurements in different background conditions are shown in Scheme 2.7. The scheme is the same for all of the AC photocurrent measurements. 
Scheme 2.7. Built-in potential measurements in different environments.

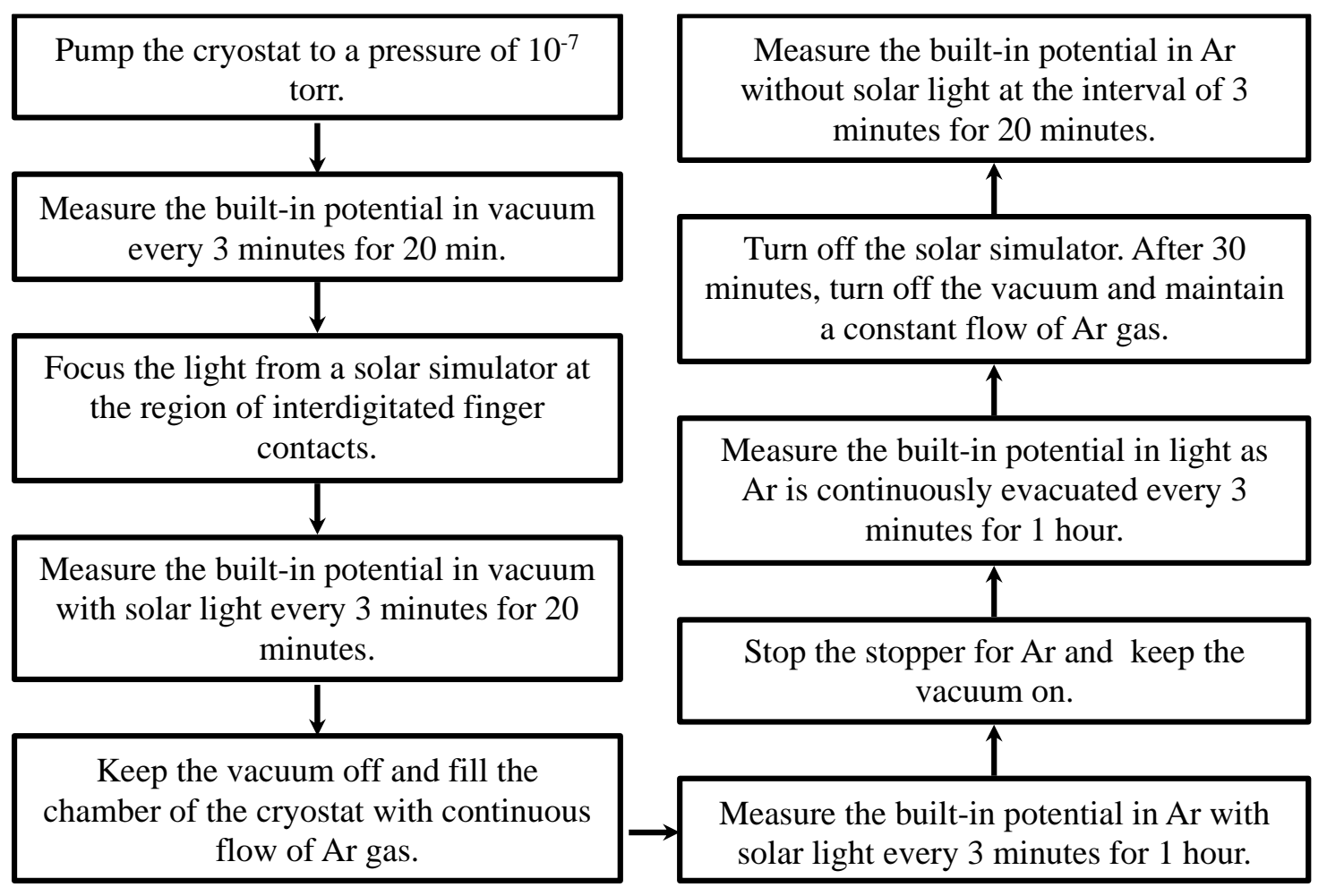

\subsection{Calculation of Average Grain Size}

The average grain sizes of all the films used in Chapter 5 were calculated using a standard ASTM E112 intercept procedure. ${ }^{108}$ The procedure consists of drawing several lines of same length on the film and counting the number of grain boundaries intersected by the test lines. If $\mathrm{L}$ is the total length of test lines, which intersect $\mathrm{N}_{\mathrm{i}}$ grain boundaries, the number of grain boundary intersections per unit length of test line, $\bar{N}_{L}$, is given by

$$
\bar{N}_{L}=\frac{N_{i}}{L / M}
$$

where $\mathrm{M}$ is the magnification. The lineal size of the grain, $\bar{l}$, is the reciprocal of $\bar{N}_{L}$. Special care should be given when counting the number of grain boundaries intersected by the test lines. When counting intercepts, segments at the end of a test line which penetrate into a grain are scored as half intercepts. When counting intersections, the end points of a test line are not intersections and are not counted except when the end appears to exactly touch 
a grain boundary, when 0.5 intersection should be scored. A tangential intersection with a grain boundary should be scored as one intersection. An intersection apparently coinciding with the junction of three grains should be scored as 1.5. With irregular grain shapes, the test line may generate two intersections with different parts of the same grain, together with a third intersection with the intruding grain. The two additional intersections are to be counted. For a particular granular film shown in Figure 2.10, there are 11 test lines each 4.53" long. $L=49.83^{\prime \prime}, M=109.2$ and $N_{i}=136$. This yields the lineal grain size of $85.22 \mu \mathrm{m}$.

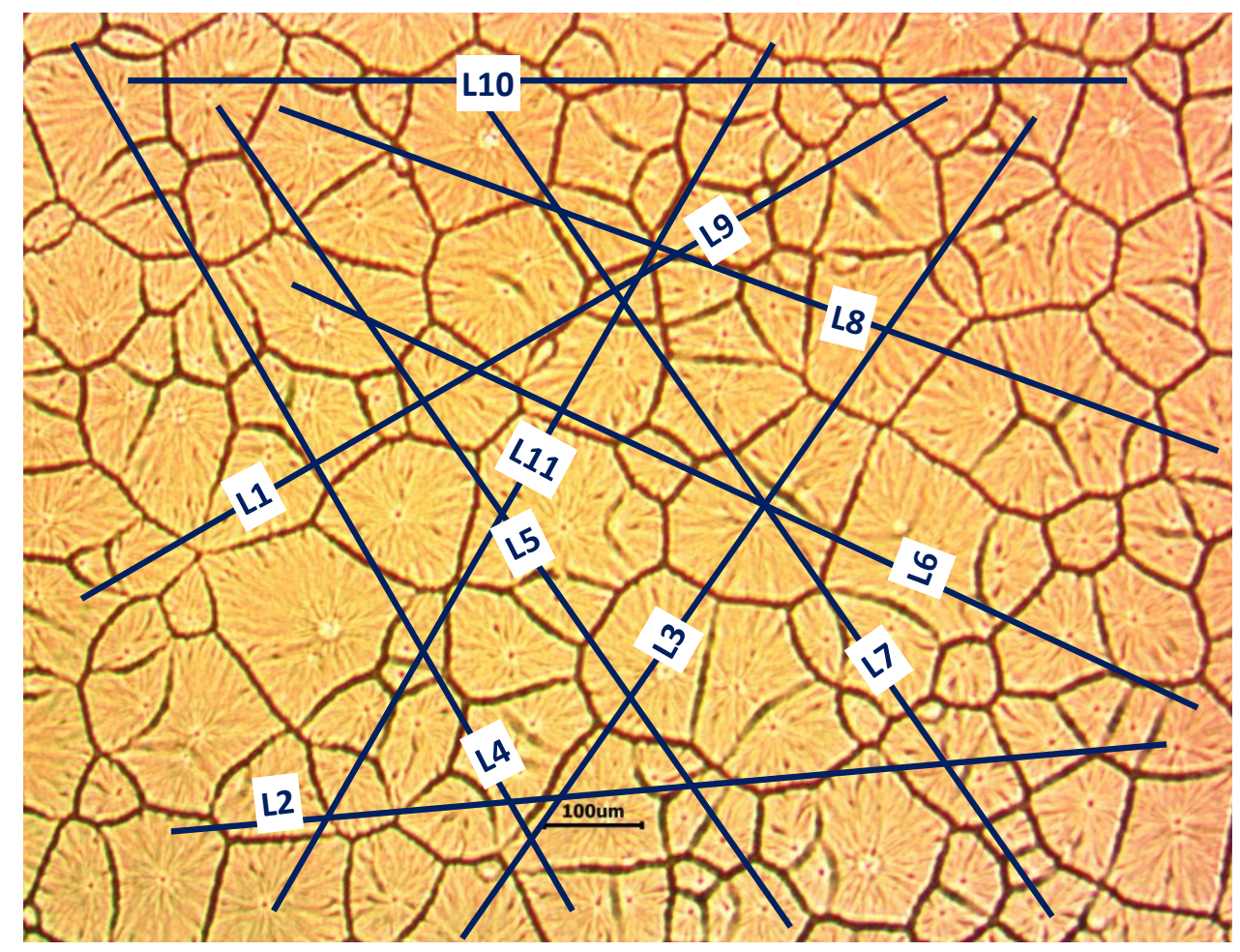

Figure 2.10. ASTM procedure for determining the average size of grains. The average grain size for this sample was found to be $85.22 \mu \mathrm{m}$. 


\subsection{Quartz Crystal Microbalance (QCM) Measurements}

A mixture of $\mathrm{MACl}$ and $\mathrm{PbI}_{2}$ in a $1.1: 1$ molar ratio was stirred at $70{ }^{\circ} \mathrm{C}$ overnight using N,N-dimethylformamide as the solvent. The solution was then spin-coated onto a fresh quartz crystal using chlorobenzene as an anti-solvent at $4000 \mathrm{rpm}$ for 30 seconds. Briefly, $80 \mu \mathrm{L}$ of the precursor solution was dropped to the center of the crystal and spun immediately at $4000 \mathrm{rpm}$. $200 \mu \mathrm{L}$ of chlorobenzene was then added to the center of the crystal after 5 seconds while it was spinning. Following spin-coating, the film was heated at $100{ }^{0} \mathrm{C}$ for 10 minutes. The quartz crystal coated with perovskite film (QCP) was mounted onto an optical cryostat and the cryostat was connected to a vacuum pump and a gas supply as shown in Figure 2.11. First the cryostat chamber was evacuated to a pressure of $10^{-7}$ torr. The resonant frequency (RF) of the QCP was measured first in the dark and then in the light until a steady RF was obtained in each case. The intensity of light was adjusted so that there was no continuous enhancement in the RF with time. This was done using a $400 \mathrm{~nm}$ long pass filter to cut off all UV radiations and adjusting the distance between the crystal and solar simulator. Vacuum was then kept off, and the cryostat chamber was filled with a continuous flow of He gas at 10 psi. The RF of QCP was measured in the dark and light alternatively three times until a steady RF was obtained in each case. This measurement was repeated with $\mathrm{N}_{2}$ and Ar gases.

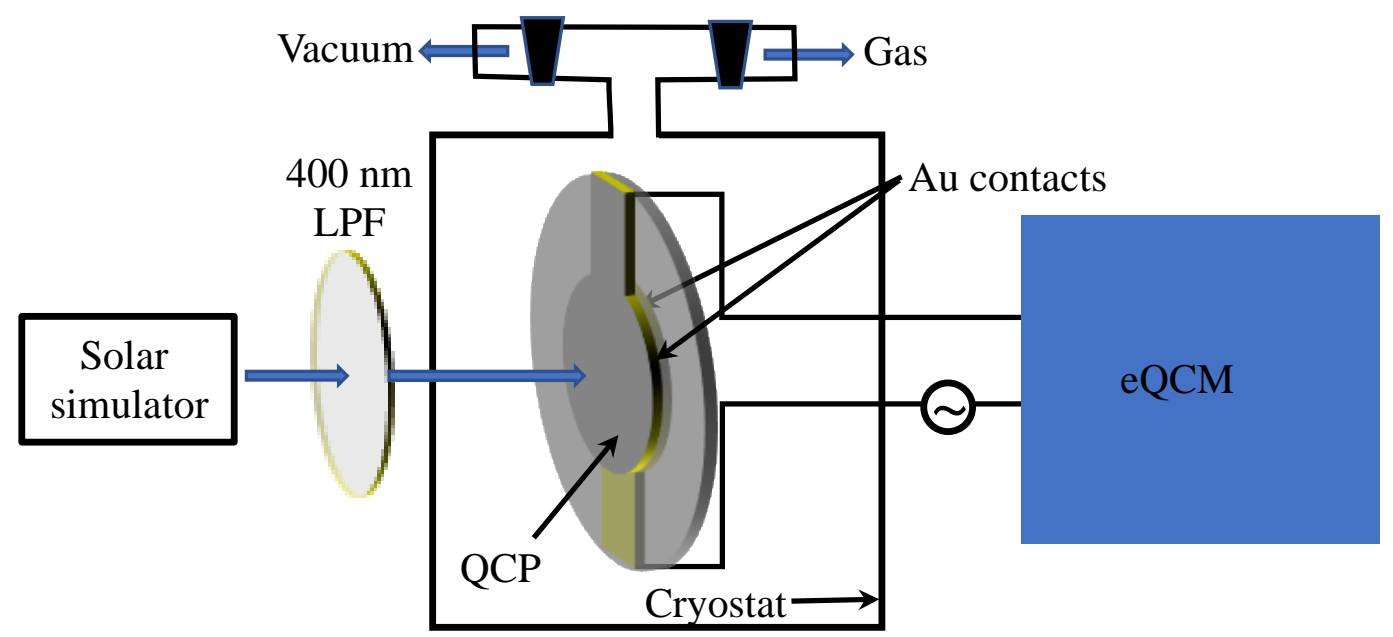

Figure 2.11. Experimental setup for QCM measurement with quartz crystal coated with perovskite film. 


\subsection{Fabrication of Perovskite Solar Cell}

ITO coated glass was used for the fabrication of mixed halide hybrid perovskite solar cell (MHHPSC). First, one end of the ITO glass was etched with zinc nano powder and 2 M hydrochloric acid three times to make sure ITO was completely removed. This is illustrated in Figure 2.12. Briefly, the ITO glass was partially covered with a tape so as to expose the area to be etched. A thin layer of paste of zinc nano powder in ethanol was applied to the area with a brush, and it was allowed to dry. Then few drops of $2 \mathrm{M} \mathrm{HCl}$ were dropped to cover the zinc film. When the reaction was over (no bubbles of hydrogen gas evolved), the surface was wiped out with a tissue paper, and the process was repeated. This was done in a fume hood. The tape was removed and the ITO glass was then cleaned by sonicating with aqueous detergent solution, acetone, ethanol and isopropanol, followed by UV/ozone treatment as mentioned above. A thin layer of PEDOT.PSS was deposited by spin-coating at $5000 \mathrm{rpm}$ for 30 seconds. The film was heated at $150{ }^{\circ} \mathrm{C}$ for 30 minutes. PEDOT.PSS coated ITO glass was heated at $180{ }^{\circ} \mathrm{C}$ for 5 minutes. On top of the PEDOT.PSS film was deposited a perovskite film by hot-casting ${ }^{16}$ the precursor solution at $4000 \mathrm{rpm}$ for $30 \mathrm{~s}$. Finally, a $60 \mathrm{~nm}$ thick film of $\mathrm{C}_{60}$ was then deposited on the perovskite film by electron beam evaporation. For this, $\mathrm{C}_{60}$ powder was pressed to a hard mass using a pestle and the deposition was done slowly using very low filament current less that $3 \mathrm{~mA}$. The top contact of gold was made by electron beam evaporation using a mask of area 0.3 $\mathrm{mm}^{2}$. The Scheme 2.8 shows the procedure for the fabrication of the perovskite solar cell. The configuration of the complete solar cell is given in Figure 2.13. 


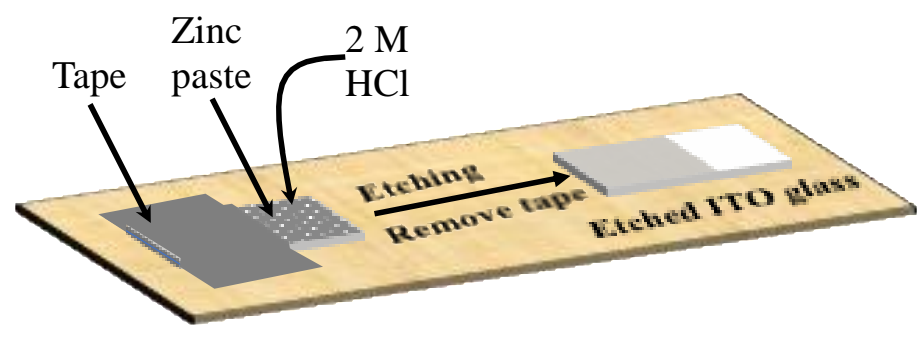

Figure 2.12. Etching of ITO using zinc paste and $2 \mathrm{M} \mathrm{HCl}$.

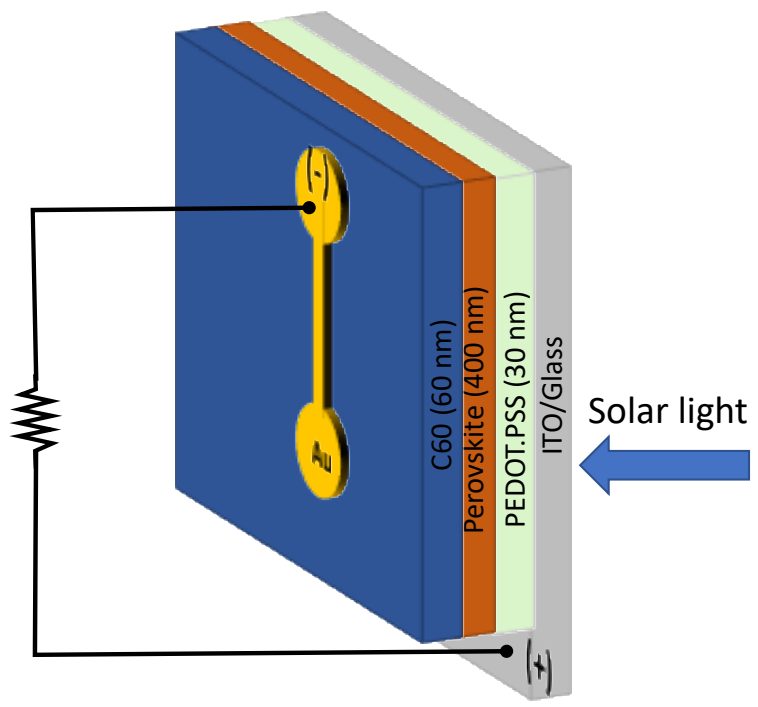

Figure 2.13. Configuration of perovskite solar cell. 
Scheme 2.8. Fabrication of perovskite solar cell

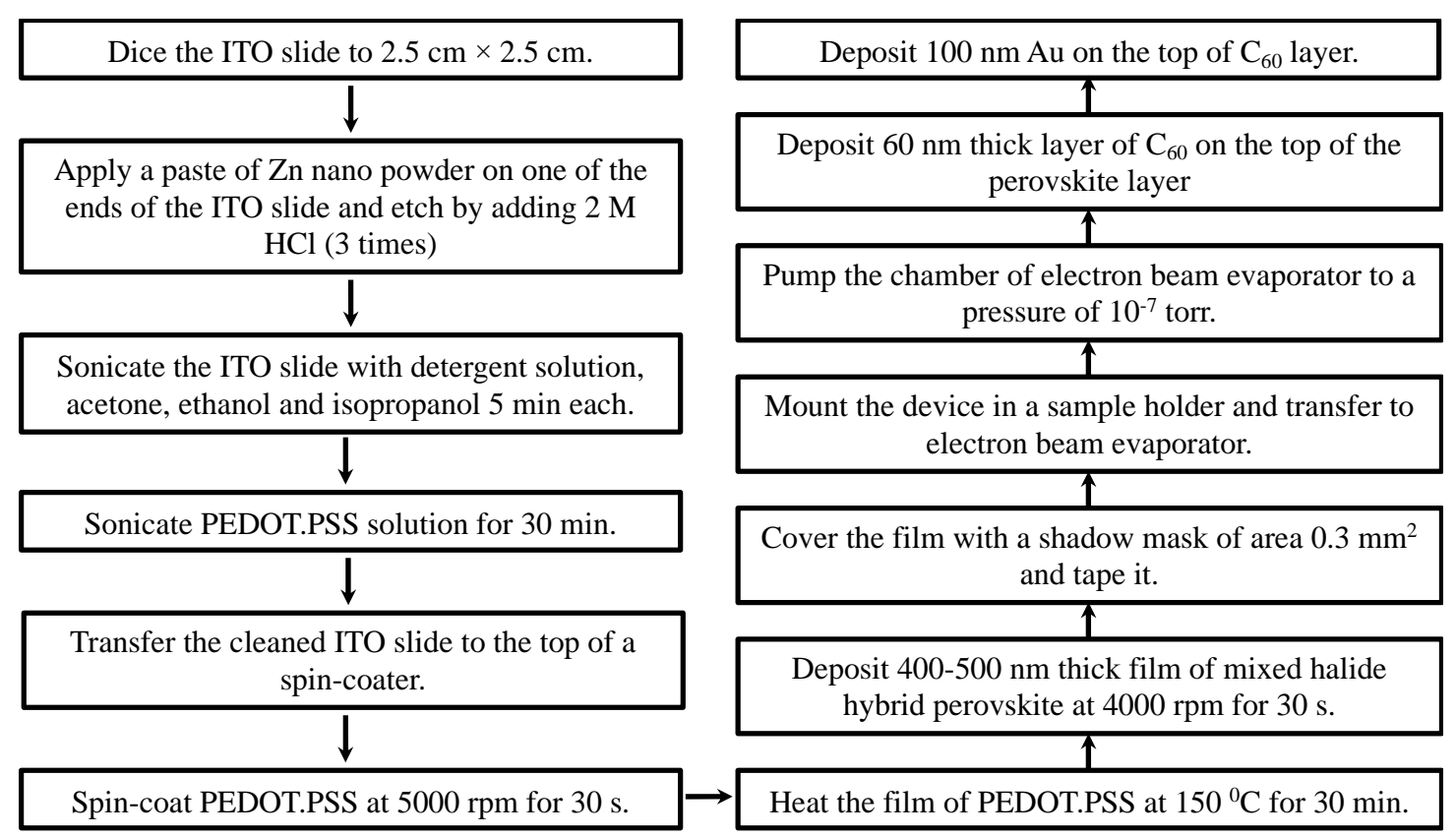

\subsection{Photoelectrochemical Measurements of Perovskite Solar Cells}

The device was mounted in an optical cryostat. A small portion of PEDOT.PSS/Perovskite/C60 film was carefully scratched with a blade to expose the ITO surface for the bottom contact and gold contact was used as the top contact. The top and bottom contacts were connected with copper wires with silver paint (Figure 2.14a). Figure $2.14 \mathrm{~b}$ shows the overall setup for photoelectrochemical measurement. The current-voltage curves were collected using a Keithley 2400 source meter under solar illumination from a tungsten lamp with a power density $82 \mathrm{~mW} \mathrm{~cm}{ }^{-2}$. The measurements were carried out in the following four sections:

(i) The device was kept in vacuum for 30 minutes and the dark photocurrent density was measured as a function of applied potential by cycling from -0.1 to $0.9 \mathrm{~V}$ at a scan rate of $10 \mathrm{mV} / \mathrm{s}$. 
(ii) The white light from a solar simulator was shone on the device (opposite to the side where the gold contacts were made) and the $\mathrm{J}-\mathrm{V}$ characteristics were measured at different times for 30 minutes.

(iii) The vacuum was discontinued, and the cryostat was then filled with a continuous flow of Ar gas. The J-V characteristics were measured at different times for $2 \mathrm{~h}$.

(iv) The flow of Ar gas was then stopped and the Ar was pumped out to create a vacuum in the chamber of the cryostat. As the Ar gas was pumped, the J-V characteristics were measured at different times for $1 \mathrm{~h}$.

(v) Finally, the light was removed and a continuous flow of Ar was maintained into the cryostat for $1 \mathrm{~h}$. The J-V characteristics were measurement in dark with Ar gas.

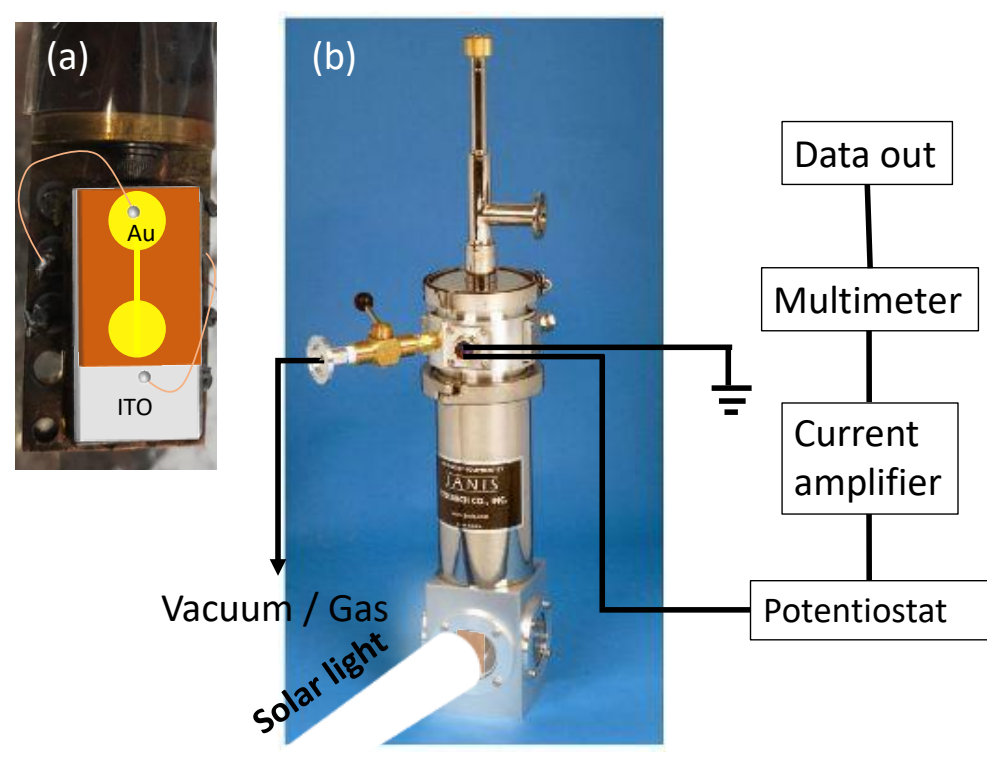

Figure 2.14. Digital pictures of the (a) mounting of the solar cell on the optical cryostat and (b) experimental setup for the J-V measurements. 


\subsection{Synthesis of Crystals of Layered and 3D Hybrid Perovskites}

2D layered hybrid perovskites with the general formula $(B A)_{2}(\mathrm{MA})_{\mathrm{n}-1} \mathrm{~Pb}_{\mathrm{n}} \mathrm{I}_{3 \mathrm{n}+1}$ and three dimensional $\mathrm{MAPbI}_{3}$ were synthesized using lead oxide, 57\% w/w HI aqueous solution, and n-butylamine following the procedure described elsewhere ${ }^{102}$ with some modifications. The details of the synthesis of the crystals of 2D and 3D hybrid perovskites are as follows.

(a) (BA) $2 \mathbf{P b I}_{4}(\mathbf{n}=1)$. A mixture of $2.232 \mathrm{~g}$ of $\mathrm{PbO}, 10 \mathrm{~mL}$ of $57 \% \mathrm{w} / \mathrm{w}$ aqueous $\mathrm{HI}$ solution, and $1.7 \mathrm{~mL}$ of $50 \% \mathrm{H}_{3} \mathrm{PO}_{2}$ was combined and heated on a hot plate with magnetic stirring under a fume hood. The temperature was increased from $100{ }^{\circ} \mathrm{C}$ to $180{ }^{\circ} \mathrm{C}$ during until a clear yellow solution was observed. In a separate beaker, $927 \mu \mathrm{L}$ of n-butylamine was added to $5.0 \mathrm{~mL}$ of $57 \% \mathrm{w} / \mathrm{w}$ aqueous $\mathrm{HI}$ in an ice bath. This was the spacer solution containing the larger organic cation salt, n-butylammonium iodide. This spacer solution was then added to the hot yellow solution of lead iodide as prepared above. The stirring was continued until the solution turned clear and yellow. It was allowed to cool on its own. Slowly, orange colored rectangular crystals started to appear. The crystals were left undisturbed for $2 \mathrm{~h}$ to allow complete crystallization. The crystals were vacuum filtered and then dried under a low-pressure environment at $40{ }^{\circ} \mathrm{C}$ for $12 \mathrm{~h}$.

(b) $(\mathbf{B A})_{2}$ (MA) $\mathbf{P b}_{2} \mathbf{I}_{7}(\mathbf{n}=2)$. A mixture of $2.232 \mathrm{~g}$ of $\mathrm{PbO}, 10 \mathrm{~mL}$ of $57 \% \mathrm{w} / \mathrm{w}$ aqueous $\mathrm{HI}$ solution, and $1.7 \mathrm{~mL}$ of $50 \% \mathrm{H}_{3} \mathrm{PO}_{2}$ was heated on a hot plate with magnetic

stirring under a fume hood. The temperature was increased from $100{ }^{\circ} \mathrm{C}$ to $180{ }^{\circ} \mathrm{C}$ during heating until a clear yellow solution was observed. $338 \mathrm{mg}$ of $\mathrm{MACl}$ was added to the clear solution and heating was continued with stirring. Initially, a black precipitate appeared which quickly dissolved upon heating and stirring rapidly to yield a clear yellow solution. In a separate beaker $624 \mu \mathrm{L}$ of n-butylamine was added to $5.0 \mathrm{~mL}$ of $57 \% \mathrm{w} / \mathrm{w}$ aqueous $\mathrm{HI}$ in an ice bath. The spacer solution of n-butylammonium iodide was then added to the hot yellow solution already described. The stirring was continued until the solution turned clear and yellow. It was then allowed to cool on its own, where scarlet colored rectangular crystals started to appear slowly over time. The solution was left undisturbed for $2 \mathrm{~h}$ to 
allow complete crystallization. The crystals were vacuum filtered and then dried under a low-pressure environment at $40{ }^{\circ} \mathrm{C}$ for at least $12 \mathrm{~h}$.

(c) $(\mathrm{BA})_{2}(\mathrm{MA})_{2} \mathrm{~Pb}_{3} \mathrm{I}_{10}(\mathrm{n}=3),(\mathrm{BA})_{2}(\mathrm{MA})_{3} \mathrm{~Pb}_{4} \mathrm{I}_{13}(\mathrm{n}=4)$ and MAPbI3. The process for the synthesis for the $2 D$ hybrid perovskites for $n=3$ and $n=4$ is the same as for $n=2$ except the amounts of methylammonium chloride and the volumes of n-butylamine taken for the spacer solution. The crystals of $\mathrm{MAPbI}_{3}$ were prepared using $675 \mathrm{mg}$ of $\mathrm{MACl}$ without the spacer solution. The masses $\mathrm{MACl}$ and the volumes of n-butylamine used for the different 2D crystals are given in Table 2.1. The overall procedure for the synthesis of $2 \mathrm{D}$ and $3 \mathrm{D}$ crystals is summarized in Scheme 2.9. Figure 2.15 shows the digital pictures of these crystals.

Table 2.1. $\mathrm{MACl}$ and n-butylamine for the synthesis of $2 \mathrm{D}$ hybrid perovskite crystals

\begin{tabular}{|c|c|c|c|}
\hline Value of $n$ & Formula & $\begin{array}{c}\text { Mass of MACl } \\
(\mathrm{mg})\end{array}$ & Volume of n-butylamine $(\mu L)$ \\
\hline 1 & $(\mathrm{BA})_{2} \mathrm{PbI}_{4}$ & 0 & 927 \\
\hline 2 & $(\mathrm{BA})_{2}(\mathrm{MA}) \mathrm{Pb}_{2} \mathrm{I}_{7}$ & 338 & 624 \\
\hline 3 & $(\mathrm{BA})_{2}(\mathrm{MA})_{2} \mathrm{~Pb}_{3} \mathrm{I}_{10}$ & 450 & 327 \\
\hline 4 & $(\mathrm{BA})_{2}(\mathrm{MA})_{2} \mathrm{~Pb}_{4} \mathrm{I}_{13}$ & 507 & 248 \\
\hline
\end{tabular}

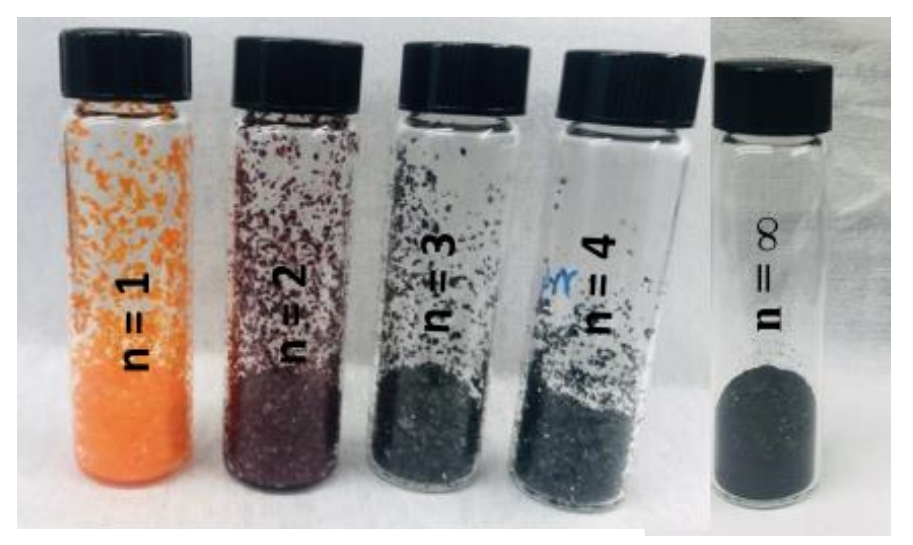

Figure 2.15. Digital picture of crystals of 2D and 3D hybrid perovskites. 
Scheme 2.9. Synthesis of crystals of $2 \mathrm{D}$ and $3 \mathrm{D}$ hybrid perovskites

1. Take $2.232 \mathrm{~g}$ of $\mathrm{PbO}$ in a round bottom flask.

2. Add $10 \mathrm{~mL}$ of $57 \% \mathrm{w} / \mathrm{w} \mathrm{HI}$ and $1.7 \mathrm{~mL}$ of $50 \% \mathrm{w} / \mathrm{w}$ aqueous $\mathrm{H}_{3} \mathrm{PO}_{2}$.

3. Increase the temperature from $100{ }^{\circ} \mathrm{C}$ to $180{ }^{\circ} \mathrm{C}$ with stirring magnetically until a clear yellow solution is observed. This gives $\mathrm{PbI}_{2}$ solution.

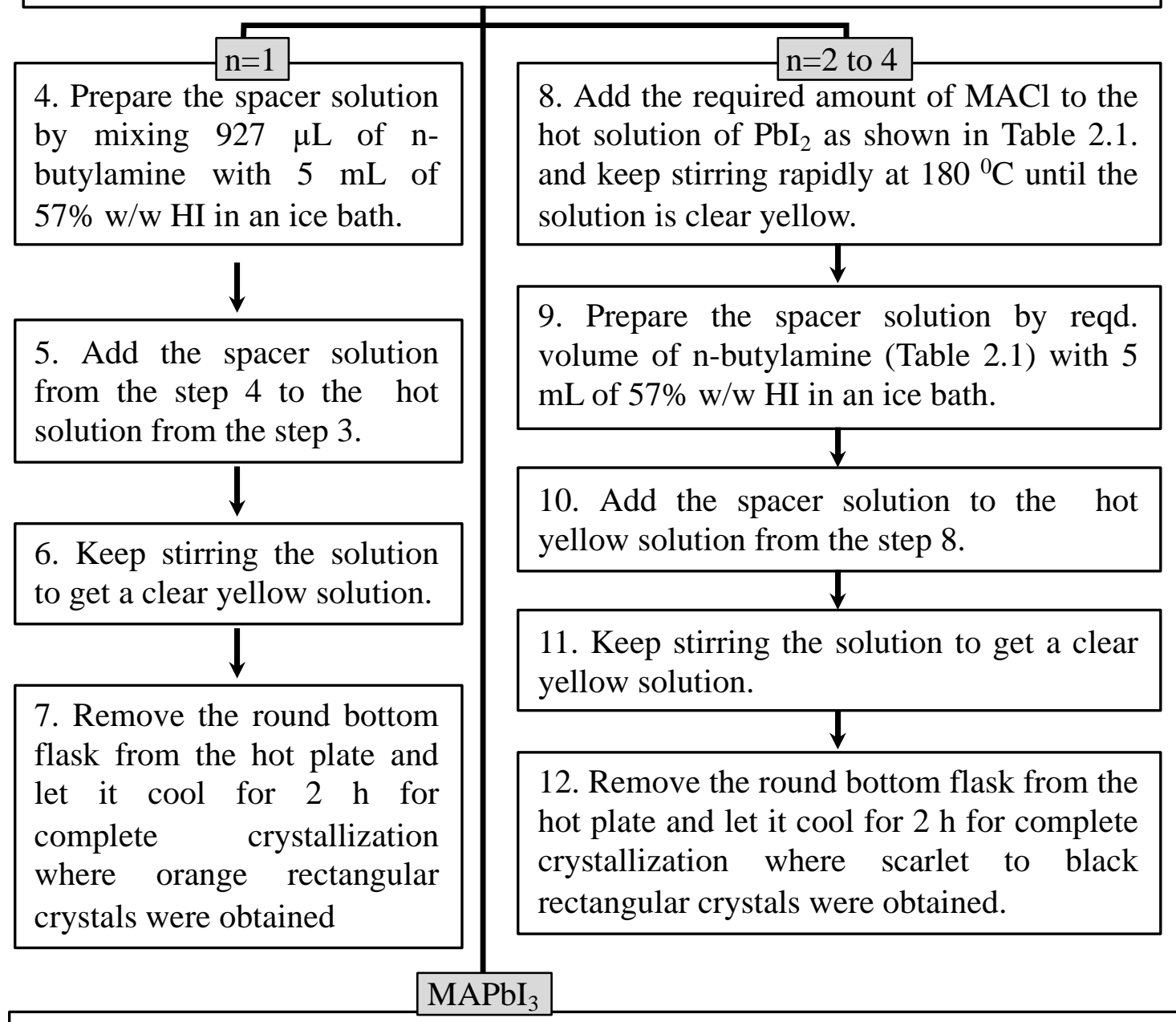

13. Add $675 \mathrm{mg}$ of $\mathrm{MACl}$ to the hot solution of $\mathrm{PbI}_{2}$ and keep stirring at $180{ }^{\circ} \mathrm{C}$ until the solution is clear yellow.

14. Remove the round bottom flask from the hot plate and let it cool for $2 \mathrm{~h}$ for complete crystallization where polyhedral crystals were obtained. 


\section{CHAPTER III}

\section{ENHANCING THE PHOTOVOLTAIC PERFORMANCE OF DYE-SENSITIZED SOLAR CELLS WITH RARE-EARTH METAL OXIDE NANOPARTICLES}

This was the first research project during my Ph.D. study. The research has been published in the Journal of the Electrochemical Society. The motivation behind this research was that the incorporation of REO microparticles increased the photovoltaic performance of DSSCs. ${ }^{52}$ In this context, we made highly reproducible and efficient DSSCs with and without incorporation of REO nanoparticles, and clearly showed that the photovoltaic performance was enhanced by the addition of REO nanoparticles. The enhancement mainly comes from the increased photocurrent. We explored the various factors responsible for the photovoltaic enhancement.

\subsection{Introduction}

Worldwide demand for energy continues to increase, and it is expected to double by 2050 and triple by the end of this century. ${ }^{109}$ Photovoltaic devices, which directly convert solar energy to electrical energy, are an increasingly important alternative to nonrenewable carbon-based energy sources. ${ }^{110}$ One recently developed photovoltaic is the DSSC, which has been promoted as a possible replacement for traditional silicon solar cells. ${ }^{111-113}$ As first reported by O'Regan and Gratzel, ${ }^{113}$ the DSSC device architecture consists of a photoanode made from dye-sensitized mesoporous titania, a counter 
electrode which is usually made from a platinum-coated transparent conducting oxide (TCO), and redox couple injected between the photoanode and counter electrode. When the device is illuminated, the electrons are photoexcited within the dye and transferred into the conduction band of $\mathrm{TiO}_{2}$ where they then diffuse to the TCO. The circuit is completed as charge from the counter electrode is drawn in to reduce the redox molecule in the electrolyte, which also replenishes electrons to the dye as it becomes oxidized. ${ }^{112}$

The percentage conversion efficiency $(\eta)$ of a DSSC is the product of the short-circuit current density $\mathrm{J}_{\mathrm{SC}}$, the open circuit potential $\mathrm{V}_{\mathrm{OC}}$, and the fill factor (FF), divided by the total solar power incident on the cell $\mathrm{P}_{\text {in. }}{ }^{43,112,114}$

$$
\eta=\frac{\mathrm{J}_{\mathrm{sc}} \times \mathrm{V}_{\mathrm{oc}} \times \mathrm{FF}}{\mathrm{P}_{\mathrm{in}}}
$$

The efficiency can be improved by increasing FF, $\mathrm{J}_{\mathrm{SC}}$, and $\mathrm{V}_{\mathrm{OC}}$. The FF can be increased by decreasing the total series resistance of the cell, ${ }^{43}$ which requires careful device design. $\mathrm{J}_{\mathrm{SC}}$ can be increased by using a dye with a broader absorption spectrum, or by increasing the amount of dye adsorbed onto the mesoporous $\mathrm{TiO}_{2} \cdot{ }^{37} \mathrm{VOC}$ can be increased by increasing the rate of electron injection or decreasing the recombination, ${ }^{43}$ or by making the electrolyte potential more positive using different redox couples or using additives. ${ }^{39}$ Attempts have also been made to improve the electrical and physical properties of the $\mathrm{TiO}_{2}$ nanoparticle acceptor, and also the stability of the cells. These include the modification of the titania with a molecular linker ${ }^{44-45}$ for greater stability, forming core/shell structures by coating with another metal oxide ${ }^{46-47}$ for improvement of all the parameters, doping titania with different metals oxides ${ }^{115}$ and metal ions ${ }^{48}$ for increasing $\mathrm{J}_{\mathrm{SC}}$ and $\mathrm{V}_{\mathrm{OC}}$, metal nanoparticles, redox inactive ionic species ${ }^{49}$ for enhancing the charge mobility and other dopants $^{50}$ for improvement in $\mathrm{J}_{\mathrm{SC}}$.

One method known to improve the efficiency of DSSCs is to dope the titania with REOs. Various mechanisms have been proposed for the observed improvement, including increased surface area, ${ }^{116-117}$ enhanced dye coverage, ${ }^{118-119}$ deep and surface trap-filling, ${ }^{120-}$ 122 band-gap narrowing, ${ }^{123}$ improvement in electron injection and transport properties, ${ }^{117}$,

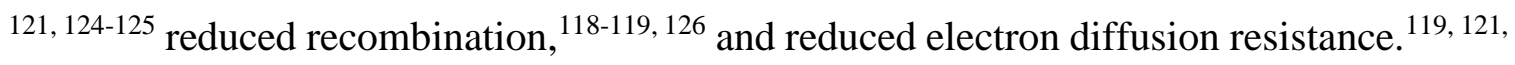


126-127 Results vary with different lanthanide species, and also among different reports. In cerium-doped $\mathrm{TiO}_{2}$ nanocrystals, cerium ions may exist at the grain boundaries or on the surface of $\mathrm{TiO}_{2}$ particles and decrease the size of $\mathrm{TiO}_{2}$ nanocrystals, thereby increasing the surface area for dye uptake. ${ }^{42,125} \mathrm{Eu}^{3+}$ and $\mathrm{Sm}^{3+}$ ions are thought to act as down-converting lanthanides to improve the efficiency of $\mathrm{DSSCs}^{128}$ while $\mathrm{Er}^{3+}$ and $\mathrm{Yb}^{3+}$ as up-converting lanthanides. ${ }^{129-130} \mathrm{NdCl}_{3}$ solution is thought to enhance electron transport efficiency by quick dye regeneration through electrolyte and reduced recombination. ${ }^{119}$ It has also been reported that doping of titania with $\mathrm{Nd}^{3+}$ ions decreases the band-gap energy and reduces the trap state density. ${ }^{121}$ Previously, it has been reported in our laboratory that the addition of large microparticles of neodymium oxide to titania enhanced the photovoltaic performance of DSSCs drastically. ${ }^{127}$ The enhancement was attributed to greater dye uptake and reduced electron transfer resistance of the mesoporous layer.

There is no doubt that doping titania with REOs is a promising approach to improve the photovoltaic performance of DSSCs, however, the amount of improvement varies among reports, and most reports lack a lucid explanation for the observed improvement. In this study, we incorporate nanoparticles of erbium (III) oxide and neodymium (III) oxide to titania simply by mechanically mixing these solid nanoparticles into the titania paste, referred to as ErNPs-Solid and NdNPs-Solid, respectively. As opposed to our previous work with microparticles, the use of nanoparticles results in DSSCs with highly reproducible characteristics. Improved efficiency in REO-doped cells is observed by our lab, and confirmed by certified measurements at a national test facility (NREL). We also perform photocurrent, impedance, IPCE, dye loading and BET analyses to identify the mechanism of the improvement. The doping of REOs increases the dye uptake by about $25 \%$, the specific surface area by about $43 \%$, while the pore diameter decreases by about $43 \%$ relative to the undoped titania. The increase in dye coverage combined with a decrease in anode impedance accounts for the improved efficiency.

\subsection{Experimental Section}

The details of the process of DSSCs fabrication with and without incorporation of REO nanoparticles into the titania are given in Chapter 2. 


\subsection{Results and Discussion}

\subsubsection{Current Density-Potential Characteristics}

The characteristic current density-potential (J-V) curves of the best doped and undoped cells are depicted in Figure 3.1a. The best ErNPs-Solid cell had an efficiency of $8.0 \%$, which is about $8 \%$ greater than the best traditional cell, and $4.7 \%$ greater than the best NdNPs-Solid cell. In the best cells, the enhancement in the photocurrent was found to be the same for both ErNPs-Solid and NdNPs-Solid cells, however, the first had a significantly greater fill factor. The detailed photoelectrochemical (PEC) parameters, conversion efficiencies $(\eta)$, short-circuit current densities $\left(\mathrm{J}_{\mathrm{SC}}\right)$, open circuit potentials $\left(\mathrm{V}_{\mathrm{OC}}\right)$ and fill factors $(\mathrm{FF})$, for each batch of three types of cells are presented in Table 3.1. The average conversion efficiencies $(\%)$ and current densities $\left(\mathrm{mA} \mathrm{cm}^{-2}\right)$ of all the batches for each type respectively are $7.01 \pm 0.16 \%$ and $13.5 \pm 0.2 \mathrm{~mA} \mathrm{~cm}^{-2}$ for traditional cells, $7.50 \pm 0.12 \%$ and $14.6 \pm 0.2 \mathrm{~mA} \mathrm{~cm}^{-2}$ for NdNPs-Solid cells, and $7.73 \pm 0.13 \%$ and $14.7 \pm 0.1$ $\mathrm{mA} \mathrm{cm}{ }^{-2}$ for ErNPs-Solid cells.

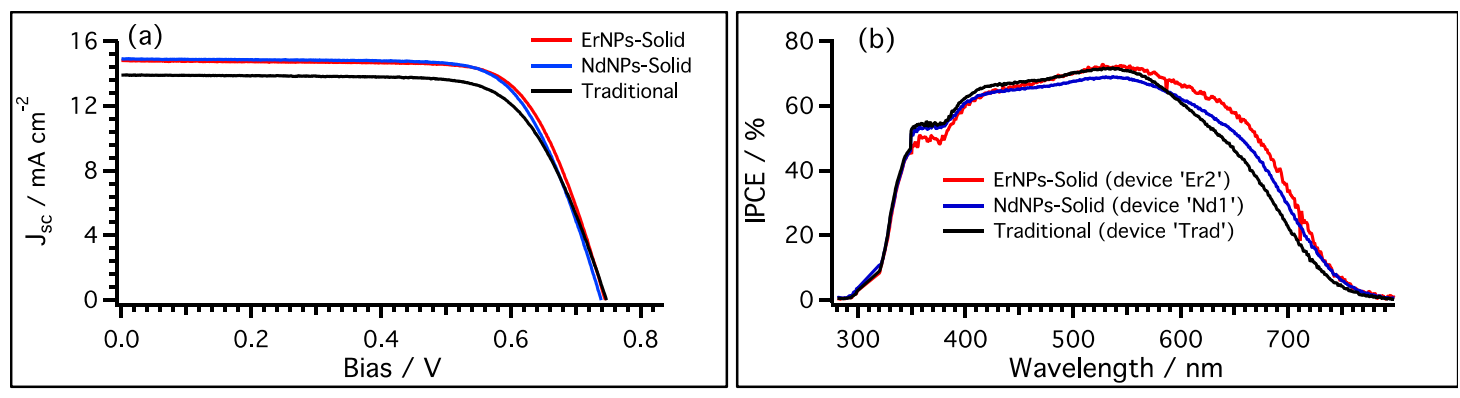

Figure 3.1. (a) JV curves of the best cells and (b) IPCE of cells measured by NREL. All these cells had an approximately $11 \mu \mathrm{m}$ thick mesoporous layer and $4-5 \mu \mathrm{m}$ scattering layer. 
Table 3.1. The cell parameters of different batches of traditional, Nd-doped and Er-doped cells.

\begin{tabular}{|c|c|c|c|c|c|c|}
\hline Cell Type & $\begin{array}{c}\text { Batch } \\
\text { No. }\end{array}$ & $\begin{array}{c}\text { No. of } \\
\text { Cells }\end{array}$ & $\mathbf{n}(\%)$ & $\begin{array}{c}\text { Jsc/mA } \\
\mathbf{c m}^{-2}\end{array}$ & Voc/V & FF \\
\hline \multirow{3}{*}{ Traditional } & 1 & 5 & $7.00 \pm 0.08$ & $13.3 \pm 0.3$ & $0.707 \pm 0.004$ & $0.742 \pm 0.006$ \\
\cline { 2 - 7 } & 2 & 4 & $6.96 \pm 0.09$ & $13.5 \pm 0.5$ & $0.692 \pm 0.008$ & $0.743 \pm 0.007$ \\
\cline { 2 - 7 } & 3 & 5 & $7.16 \pm 0.07$ & $13.4 \pm 0.4$ & $0.732 \pm 0.004$ & $0.731 \pm 0.005$ \\
\hline \multirow{3}{*}{ NdNPs- } & 1 & 5 & $7.52 \pm 0.09$ & $14.5 \pm 0.4$ & $0.703 \pm 0.004$ & $0.734 \pm 0.009$ \\
\cline { 2 - 7 } Solid & 2 & 5 & $7.37 \pm 0.19$ & $14.7 \pm 0.5$ & $0.691 \pm 0.010$ & $0.727 \pm 0.006$ \\
\cline { 2 - 7 } & 3 & 3 & $7.66 \pm 0.08$ & $14.4 \pm 0.3$ & $0.746 \pm 0.005$ & $0.716 \pm 0.003$ \\
\hline \multirow{2}{*}{ ErNPs- } & 1 & 5 & $7.73 \pm 0.08$ & $14.7 \pm 0.2$ & $0.721 \pm 0.011$ & $0.726 \pm 0.012$ \\
\cline { 2 - 7 } Solid & 2 & 5 & $7.58 \pm 0.08$ & $14.6 \pm 0.3$ & $0.738 \pm 0.007$ & $0.704 \pm 0.006$ \\
\cline { 2 - 7 } & 3 & 3 & $7.94 \pm 0.04$ & $14.8 \pm 0.2$ & $0.746 \pm 0.008$ & $0.716 \pm 0.011$ \\
\hline
\end{tabular}

Another batch of cells was prepared and sent to the National Renewable Energy Laboratory (NREL) in Colorado for certification. The PEC parameters were measured after 48 hours. The details of all the cells are provided in Figure 3.2 and Table 3.2. The ErNPsSolid showed an efficiency of $7.3 \%$ with a current density of $13.5 \mathrm{~mA} \mathrm{~cm}{ }^{-2}$, which is about $14 \%$ more efficient than the traditional cell. The NdNPs-Solid cell was found to be about $5 \%$ more efficient than the traditional cell. The cells might have degraded some during the 48 hours before measurement. Our results are in very close agreement with the NREL results considering the PEC parameters were measured after 48 hours in the NREL lab but within 4 hours in our lab. The IPCE of the cells measured by the NREL lab are shown in Figure 3.1b. Relative to the Traditional cells, doped cells have higher photocurrents at longer wavelengths, which might be an indication of the up-converting characteristics of the $\mathrm{Er}_{2} \mathrm{O}_{3}$ and $\mathrm{Nd}_{2} \mathrm{O}_{3}$. 


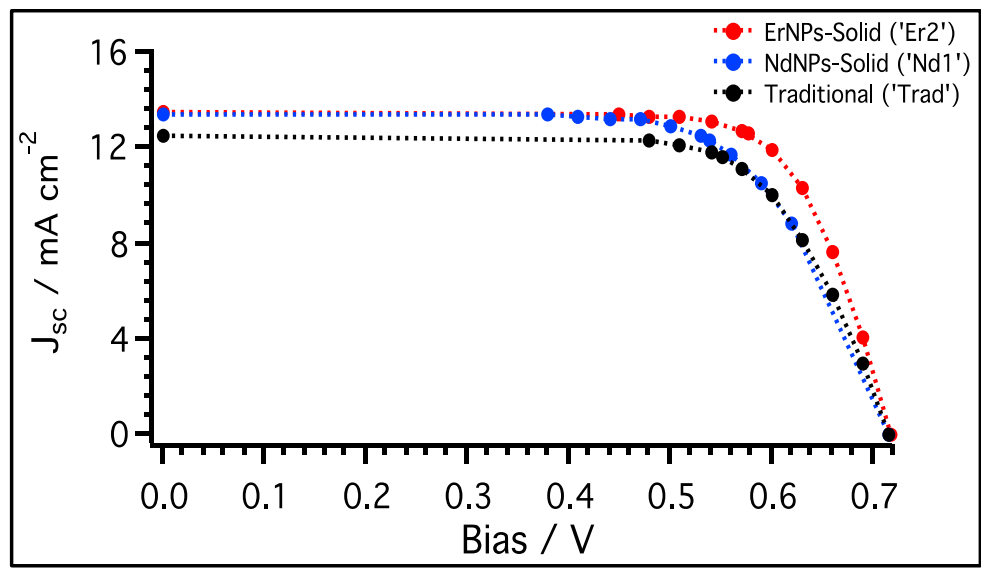

Figure 3.2. JV plots of cells measured by NREL after 48 hours. The corresponding quantum efficiency plot is given in Figure 3.1b.

Table 3.2. The cell parameters of cells measured by NREL

\begin{tabular}{|l|c|c|c|c|}
\hline \multicolumn{1}{|c|}{ Device Name } & $\mathbf{n}(\boldsymbol{\%})$ & $\mathbf{J}_{\text {sc }} / \mathbf{m A ~ \mathbf { ~ c m } ^ { - 2 }}$ & $\mathbf{V}_{\text {oc }} / \mathbf{V}$ & FF \\
\hline Traditional (Trad) & 6.40 & 12.5 & 0.715 & 0.717 \\
\hline NdNPs-Solid (Nd1) & 6.62 & 13.4 & 0.715 & 0.689 \\
\hline ErNPs-Solid (Er2) & 7.30 & 13.5 & 0.717 & 0.749 \\
\hline
\end{tabular}

The UV/VIS spectra of various doped and undoped titania films on FTO and of 0.3 mM C101 dye are shown in Figure 3.3. We did not find any remarkable change in the absorption spectra of titania due to REO-doping (Figure 3.2a), however, the characteristic peaks for REO are present in the spectra. The C101 dye has strong absorption peaks (Figure 3.3b) at about 550, 400 and $350 \mathrm{~nm}$, consistent with Ru-based dyes. These features do not show up specifically in the IPCE measurements, consistent with other reports. 

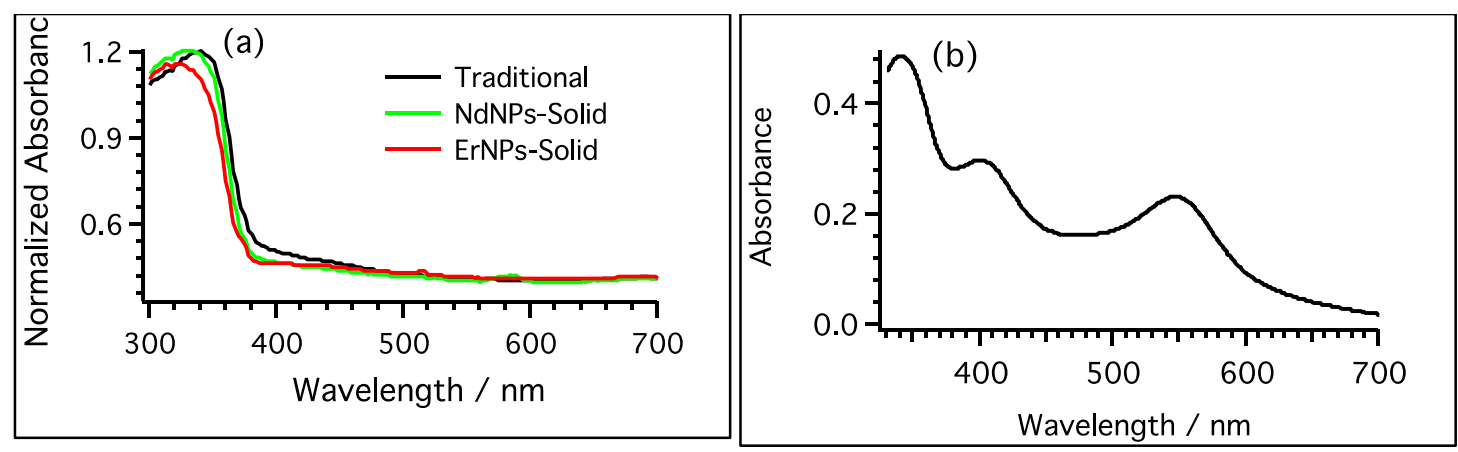

Figure 3.3. UV/Vis spectrum of (a) doped and undoped titania on FTO and (b) C101 dye.

Figure 3.4a-d show efficiencies, short-circuit current densities, open circuit potentials (OCPs) and fill factors (FFs) of the individual cells. The OCPs and FFs of the cells are provided in Figure 3.4c-d. We did not get a clear difference between doped and undoped cells for OCPs and FFs.
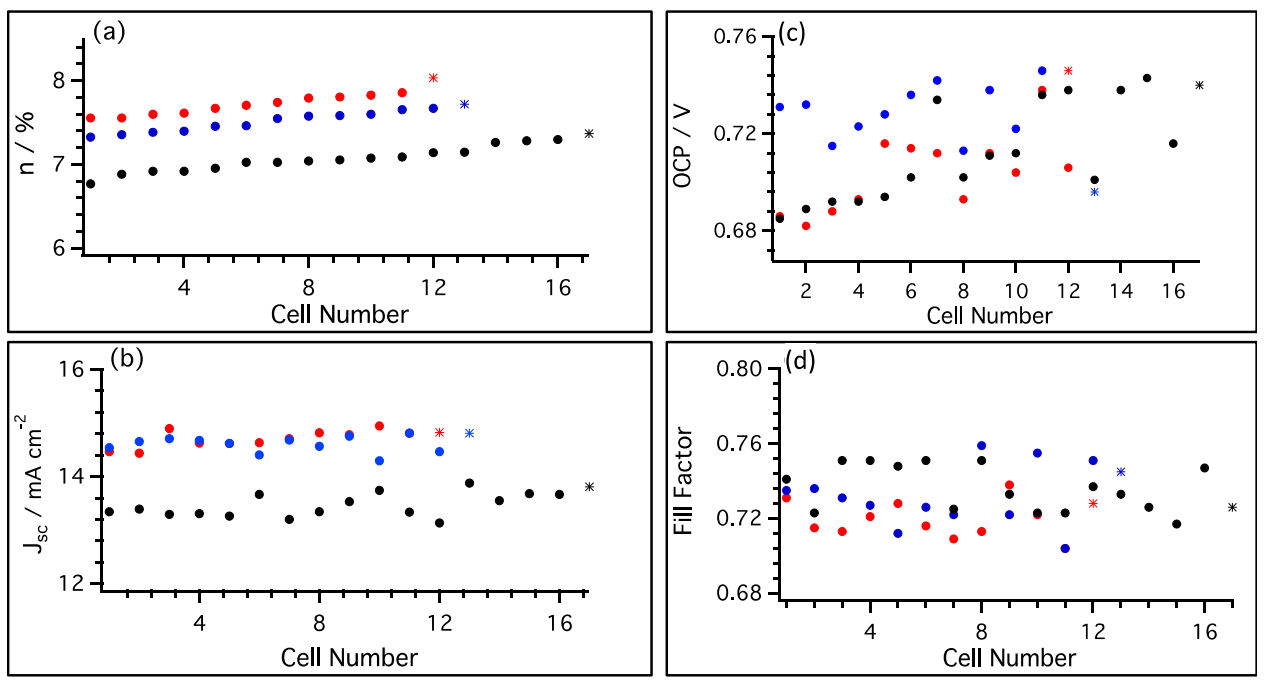

Figure 3.4. The parameters of individual cells (a) efficiency, (b) short-circuit current density, (c) open circuit potentials, and (d) fill factors. $\bullet$ : ErNPs-Solid cells, $\bullet:$ NdNPsSolid cells, $\bullet$ : Traditional cells, and $* / * / *$ : corresponding to the best cells. 


\subsubsection{Electrochemical Impedance Spectroscopy}

Next, Electrochemical Impedance Spectroscopy (EIS) was used to investigate the effect of REO nanoparticles on the charge transfer characteristics of the cells. In dark under the application of a forward bias, there is no involvement of dye, and the applied bias drives electrons through the mesoporous doped or undoped $\mathrm{TiO}_{2}$ network and reduces $\mathrm{I}_{3}^{-}$to $3 \mathrm{I}^{-}$ while at the same time there is oxidation of $3 \mathrm{I}^{-}$to $\mathrm{I}_{3}^{-}$at the counter electrode. ${ }^{131}$ In this case the impedance is mostly due to the mesoporous film of doped or undoped $\mathrm{TiO}_{2}$. The radius of the middle semicircle of the Nyquist plot represents the charge transfer resistance of the mesoporous film. Figure 3.5 shows a Nyquist plot of doped and undoped cells. The impedance of ErNPs-Solid cell is about $42 \Omega$ which is about $27.6 \%$ lower than that of the traditional cell and about $14.3 \%$ lower than that of the NdNPs-Solid cell. Conductivity measurements were performed both in dark and light. REO nanoparticles increased the conductivity of the titania significantly, which is consistent with the EIS data. The full details of the solid-state conductivity of REO-doped titania films are described in Chapter IV.

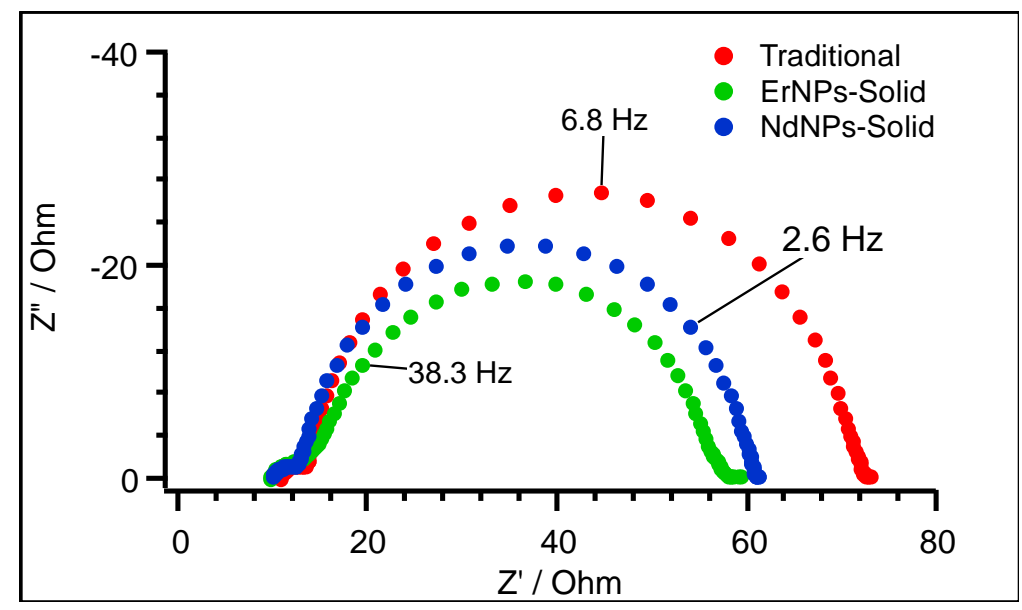

Figure 3.5. Nyquist plot of the cells shown in Figure 3.1a. 


\subsubsection{Optimization of Concentration of REO Nanoparticles and Dye Loading}

NdNPs and ErNPs were doped at different concentrations into DSL 18 NR-T titania paste and 3-4 sandwich cells were fabricated for each type of cell. Figure 3.6a shows a maximum in the efficiency for $2 \%$ concentration for NdNPs-Solid cells and $1.0 \%$ concentration for ErNPs-Solid cells. Here, \% concentration refers to \% by weight of NPs relative to the weight of NPs plus the weight of paste. The efficiency for each concentration is the average of all the cells made at that concentration. To explore the enhancement of the photovoltaic performance of REO doped cells, we studied the amount of dye adsorbed by the films having the different concentrations of REO nanoparticles. Figure 3.6b shows the dye adsorption as a function of REO concentration. The maximum adsorption of dye was obtained for doping levels of $1 \% \mathrm{Er}_{2} \mathrm{O}_{3}$ and $2 \% \mathrm{Nd}_{2} \mathrm{O}_{3}$ nanoparticles, which correlates well with the optimized concentration of REO for the best photovoltaic performance.

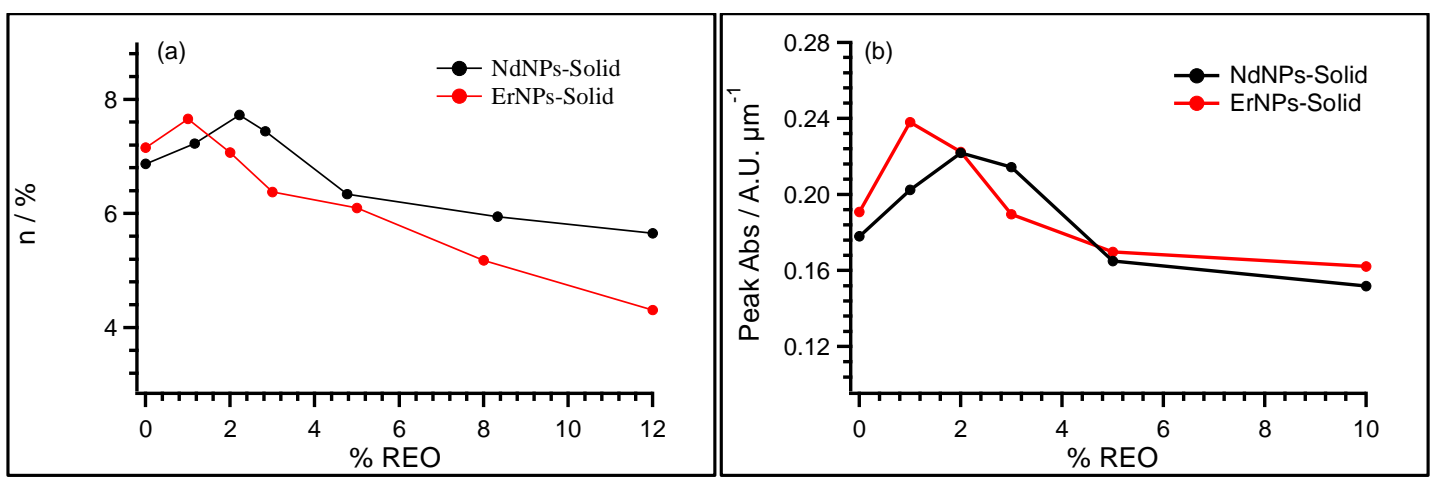

Figure 3.6. (a) Optimization of concentration of REO nanoparticles for the best photovoltaic performance of DSSCs. These cells had a $7 \mu \mathrm{m}$ thick mesoporous layer and a 4-5 $\mu \mathrm{m}$ thick scattering layer. (b) Dye absorbance as a function of concentration of REO. These photoanodes had a $7 \mu \mathrm{m}$ thick mesoporous film only. 


\subsubsection{Morphology of Doped and Undoped Films}

The images of the films of doped titania in optimized concentration and undoped titania taken with different microscopes are provided in Figures 3.7 and 3.8. The optical images in Figure 3.7 (first row) showed that in doped photoanodes, there are aggregates of REO nanoparticles in some regions. The AFM images in Figure 3.7 (middle row) showed that relative to the undoped film, there is enhancement in the roughness of the film by REO-doping, which is about $30 \%$ higher for $1 \%$ Er-doped and about $45 \%$ higher for $2 \%$ Nd-doped films. It is based on an RMS roughness measurement in the AFM software. The corresponding Scanning Electron Microscopy (SEM) images of doped films at the optimized concentration of REO nanoparticles and undoped film are presented in Figure 3.8. The SEM images show the nanostructures of pristine titania and REO doped titania. 

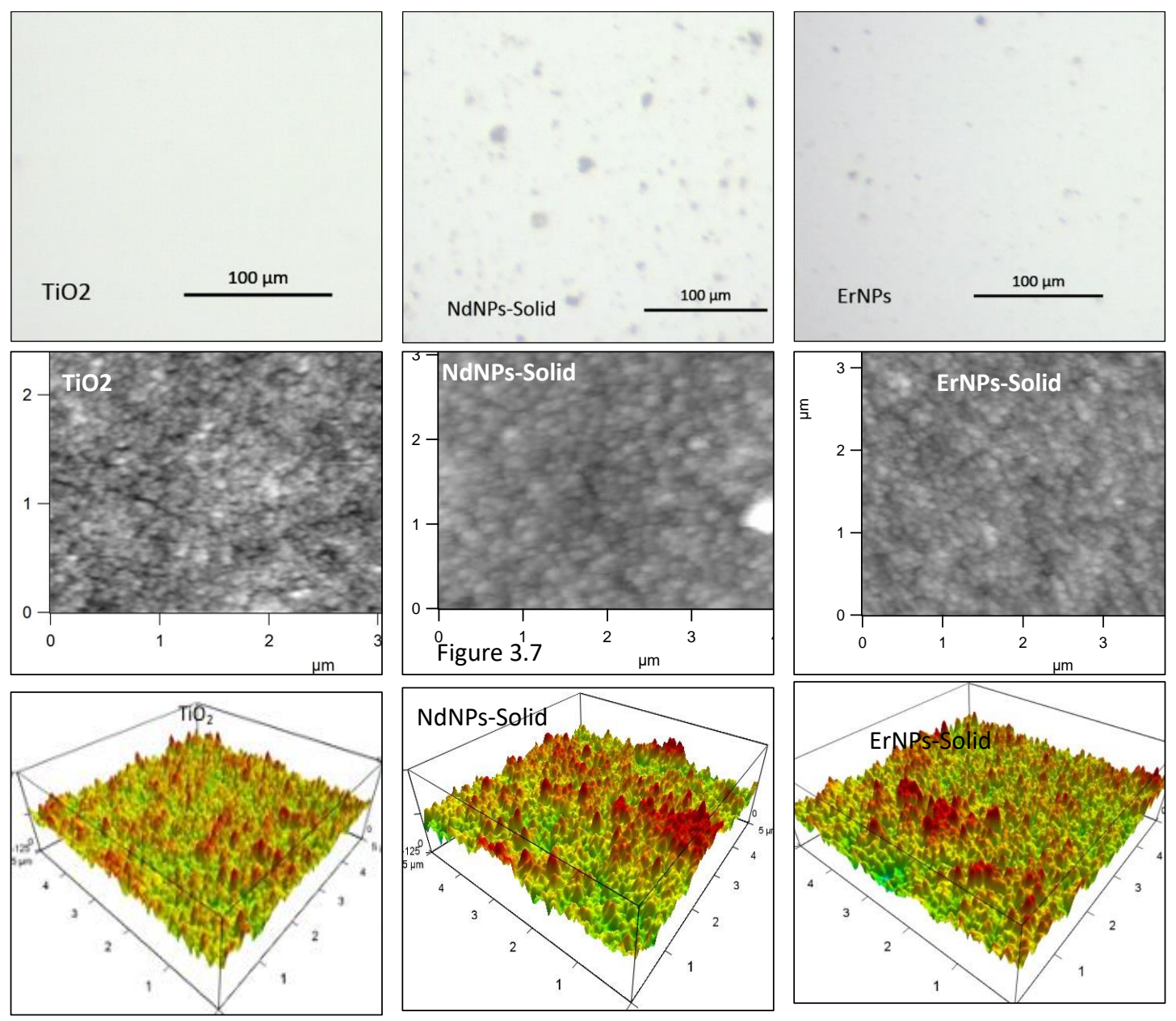

Figure 3.7. Optical images (first row) and AFM Image (second row) and 3D images (last row) of doped and undoped photoanodes at the optimized doping levels. 

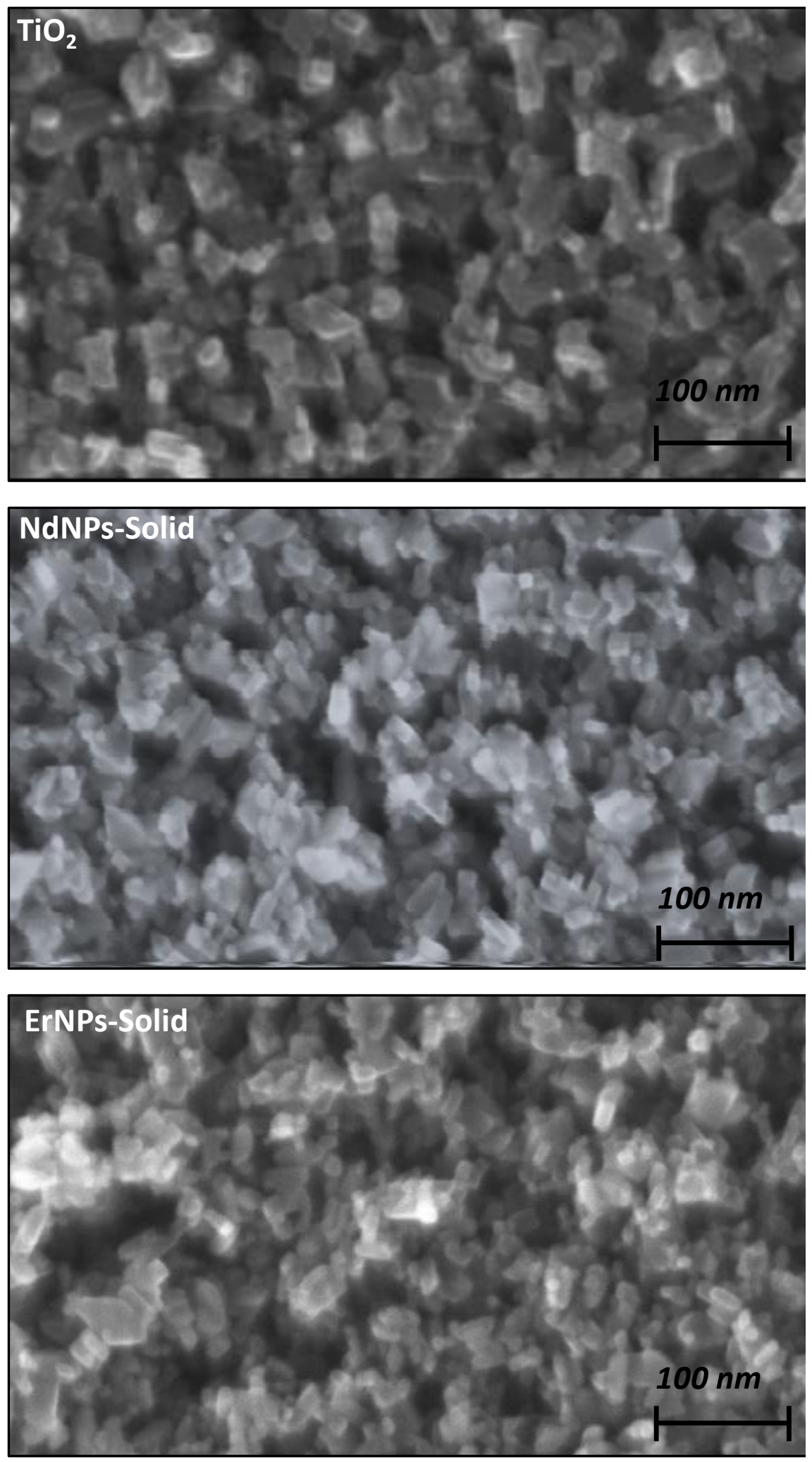

Figure 3.8. SEM images of undoped and doped films under optimized conditions. 


\subsubsection{BET Analysis of Doped and Undoped Titania}

We then measured the BET surface area and porosity of both undoped and REOdoped films using the optimized concentrations of REO. Figure 3.9a-b are the BET adsorption isotherms of doped and undoped films. Relative to undoped titania, REO-doped films are found to show higher adsorption of nitrogen gas. The specific surface area and porosity of undoped, $\mathrm{Er}_{2} \mathrm{O}_{3}$-doped and $\mathrm{Nd}_{2} \mathrm{O}_{3}$-doped titania for two runs are provided in Table 3.3. The BET analysis showed that the greater adsorption of dye for doped titania is attributed to the greater surface area available for dye adsorption. The table reveals that the pore diameter in the film is decreased while doping $\mathrm{TiO}_{2}$ with $\mathrm{REO}$. We speculate that it might be due to the insertion of REO nanoparticles in the space between the titania grains.
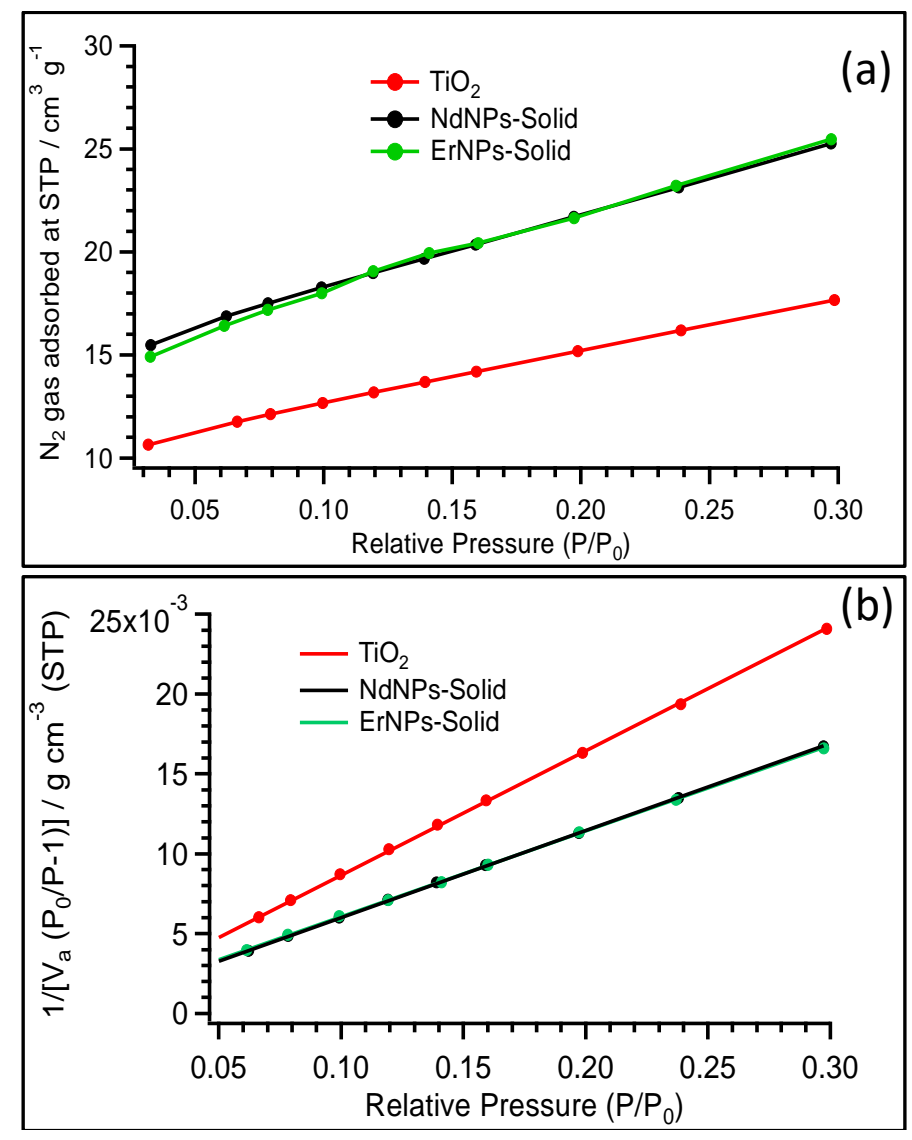

Figure 3.9. (a) $\mathrm{N}_{2}$ gas adsorbed at $\mathrm{STP}$, and (b) BET adsorption isotherms for undoped and doped titania. Here $\mathrm{V}_{\mathrm{a}}$ is the volume of gas adsorbed. 
Table 3.3. BET analysis of doped and undoped titania

\begin{tabular}{|l|c|c|c|c|}
\hline \multirow{2}{*}{ Samples } & \multicolumn{2}{|c|}{ Specific area $\left(\mathrm{m}^{2} / \mathrm{g}\right)$} & \multicolumn{2}{c|}{ Porosity $(\mathrm{nm})$} \\
\cline { 2 - 5 } & Run 1 & Run 2 & Run 1 & Run 2 \\
\hline Undoped titania & 55.3 & 53.3 & 26.3 & 26.9 \\
\hline $\mathrm{Er}_{2} \mathrm{O}_{3}$-doped titania & 79.1 & 77.3 & 18.1 & 18.4 \\
\hline $\mathrm{Nd}_{2} \mathrm{O}_{3}$-doped titania & 78.3 & 76.7 & 19.4 & 19.6 \\
\hline
\end{tabular}

\subsection{Conclusions}

The doping of mesoporous $\mathrm{TiO}_{2}$ with both $\mathrm{REO} \mathrm{Er}_{2} \mathrm{O}_{3}$ and $\mathrm{Nd}_{2} \mathrm{O}_{3}$ nanoparticles showed significant improvement in the photovoltaic performance of DSSCs. The contributing factors for the enhancement by REO-doping are the increased dye loading due to the increased surface area (as reported elsewhere), ${ }^{119,}{ }^{132}$ and the enhanced electron transport efficiency (due to higher conductivity). $\mathrm{Er}_{2} \mathrm{O}_{3}$-doped $\mathrm{TiO}_{2}$ films exhibited the best photovoltaic performance, which might also be due to the increased rate of excited-state electron injection and decreased recombination of injected electrons. ${ }^{133}$ REO-doped in $\mathrm{TiO}_{2}$ might deactivate surface and deep $\mathrm{TiO}_{2}$ trap states (the details about trapping and detrapping will be described in a later report), improve electron transport rate and solar cell efficiency. ${ }^{121}$ With reference to the IPCE data measured by NREL, we observed a higher photocurrent at longer wavelengths, which might give some evidence for the up-converting characteristics of $\operatorname{Er}(\mathrm{III})$ - and $\mathrm{Nd}(\mathrm{III})$-oxides. 


\section{CHAPTER IV}

\section{EFFECT OF RARE-EARTH METAL OXIDE NANOPARTICLES ON THE CONDUCTIVITY OF NANOCRYSTALLINE TITANIUM DIOXODE: AN ELECTRICAL AND ELECTROCHEMICAL APPROACH}

This research work has been published in the Journal of Physical Chemistry C. ${ }^{134}$

\subsection{Introduction}

The unique properties of nanocrystalline titanium dioxide (NTD) make it extremely useful for applications in photocatalysis, energy generation and energy storage. ${ }^{135-136}$ Advantages include a large bandgap $(\sim 3.2 \mathrm{eV})$, high conduction band edge energy, ${ }^{8}$ chemical and mechanical stability, efficient electron-accepting capability, ${ }^{137}$ and high redox activity in the illuminated condition. ${ }^{138}$ NTD and NTD-based photocatalytic systems are widely used for the destruction of environmental pollutants, or for the killing of harmful bacteria and cancer cells. ${ }^{139-144}$ NTD materials have the ability to accumulate a large number of injected electron charges in the solid matrix ${ }^{145}$ and can function as supercapacitors for energy storage applications. ${ }^{146}$ They also find application as the electron-conducting phase in third generation photovoltaics like perovskite solar cells, ${ }^{147-}$ ${ }^{150}$ DSSCs, ${ }^{20,113-114,151-155}$ QDSCs, ${ }^{156-157}$ and inorganic solid-state solar cells. ${ }^{156}$ 
Lattice defects, such as Ti interstitials, oxygen vacancies, and grain boundaries in nanostructured $\mathrm{TiO}_{2}$ produce a high density of electronic mid-band gap states that trap charges from the titania conduction band..$^{8,158-159}$ While the states near the conduction band edge, called shallow trap states, are in equilibrium with extended states and do not necessarily inhibit electron transport through mesoporous titania, the states deep in the band gap are slow to release trapped electrons, and thus limit electron mobility. ${ }^{160}$ In DSSC applications, these deep gap states act as recombination centers and limit solar cell efficiency. ${ }^{161}$ In photocatalysts, the trap states capture the electrons responsible for catalytic activity and degrade the catalytic performance. ${ }^{135,162}$

REOs have been used as dopants for titania to enhance photocatalytic activity ${ }^{163-166}$ and photovoltaic performance of DSSCs. ${ }^{119,}$ 125, 167-169 Various mechanisms have been proposed for the observed improvement in DSSCs, however, in most of the reports the effects are not quantitatively reproducible or thoroughly studied, resulting in a lack of clear understanding. In our previous report, ${ }^{170}$ described in Chapter 3, we demonstrated a consistent efficiency improvement in DSSCs through REO doping and explained the improvement as being due in part to a decrease in impedance of the titania electrode caused by the filling of the deep trap states by the REO $\mathrm{f}$-state electrons. This paper explores the influence of REO-doping on the conductivity of mesoporous titania by using cyclic voltammetry (CV), chronoamperometry (CA), and electrochemical impedance spectroscopy (EIS) to characterize the energy distribution of electronic states and trapping kinetics. ${ }^{145,160-161,171-172}$ We find evidence that electrons from the REO's valence band or photoexcited f-states are donated to fill the deepest gap states, reducing the trapping probability and improving both light and dark conductivity. This in turn provides an explanation for the improved performance of REO-doped titania solar cells and photocatalysts. 


\subsection{Experimental Section}

Dyesol 18 NR-T paste was used without any modification for all undoped samples. The photoanodes made using this paste directly were named as 'Undoped'. The paste for doped titania was prepared as described in Chapter 2 by mechanically mixing $\operatorname{Er}_{2} \mathrm{O}_{3}$ and $\mathrm{Nd}_{2} \mathrm{O}_{3}$ nanoparticles into titania paste at $1 \%$ and $2 \%$ by weight of titania paste, respectively. These pastes were used to make films for electrical and electrochemical measurements by screen-printing.

Gold electrodes were sputter-deposited on an oxidized silicon wafer and a $10 \mu \mathrm{m}$ gap was etched into the gold to make electrical contacts for the electrical conductivity measurements. A $7 \mu \mathrm{m}$ thick film of doped/undoped $\mathrm{TiO}_{2}$ paste was screen-printed across the gap. These films were sintered in a Barnstead Thermolyne 1300 furnace as described in Figure 2.1. Following sintering, the films were allowed to cool to room temperature gradually inside the furnace. The two-terminal devices were mounted in an optical cryostat with two-probe electrical connections made by copper wires silver painted to the gold pads on either side of the gap. The current-voltage characteristics were measured in dark and light by applying a bias from 0 to $0.5 \mathrm{~V}$ at a scan rate of $10 \mathrm{mV} / \mathrm{s}$ and measuring the current. The light source was a $100 \mathrm{~W}$ tungsten lamp with an intensity of $85 \mathrm{~mW} \mathrm{~cm}^{-2}$ at the sample. Prior to each dark scan, the device was kept in the dark for 5 minutes. Prior to each light scan, the device was exposed to the light for 1 minute at zero bias.

The working electrodes used in the electrochemical measurements were fabricated by screen-printing doped and undoped $\mathrm{TiO}_{2}$ onto pre-cleaned FTO substrates. These electrodes were then sintered in a Barnstead Thermolyne 1300 furnace and allowed to cool to room temperature gradually inside the furnace. The open exposed areas of the FTO that weren't screen printed on were painted with epoxy and cured with UV light for 30 minutes to block these regions of bare FTO so that the electrochemical measurement reflected the doped and undoped titania films only. A three-electrode system was used for all measurements, including a platinum wire counter electrode, $\mathrm{Ag} / \mathrm{AgCl}(3 \mathrm{M} \mathrm{KCl})$ reference

electrode, and the doped/undoped titania screen-printed FTO glass (epoxy blocked) as the 
working electrode (Figure 4.1). An aqueous solution of $0.1 \mathrm{M} \mathrm{KOH}$ was used as electrolyte. The electrochemical measurements were performed using a CH Instruments (Austin, TX) CHI 660A electrochemical workstation. For cyclic voltammetry (CV), the potential was applied from $1.5 \mathrm{~V}$ to $-0.5 \mathrm{~V}$ vs the reversible hydrogen electrode (RHE) (converted from $\mathrm{Ag} / \mathrm{AgCl}$ ) and the scan rates ranged from $5 \mathrm{mV} / \mathrm{s}$ to $600 \mathrm{mV} / \mathrm{s}$. For electrochemical impedance spectroscopy (EIS), an ac signal of $10 \mathrm{mV}$ amplitude at $100 \mathrm{~Hz}$ was applied on top of a DC voltage ranging from $0.2 \mathrm{~V}$ to $-0.4 \mathrm{~V}$ vs RHE. For chronoamperometry (CA), the potential was stepped from $0.4 \mathrm{~V}$ to $-0.2 \mathrm{~V}$ vs RHE and the current was measured as a function of time for the three different films.

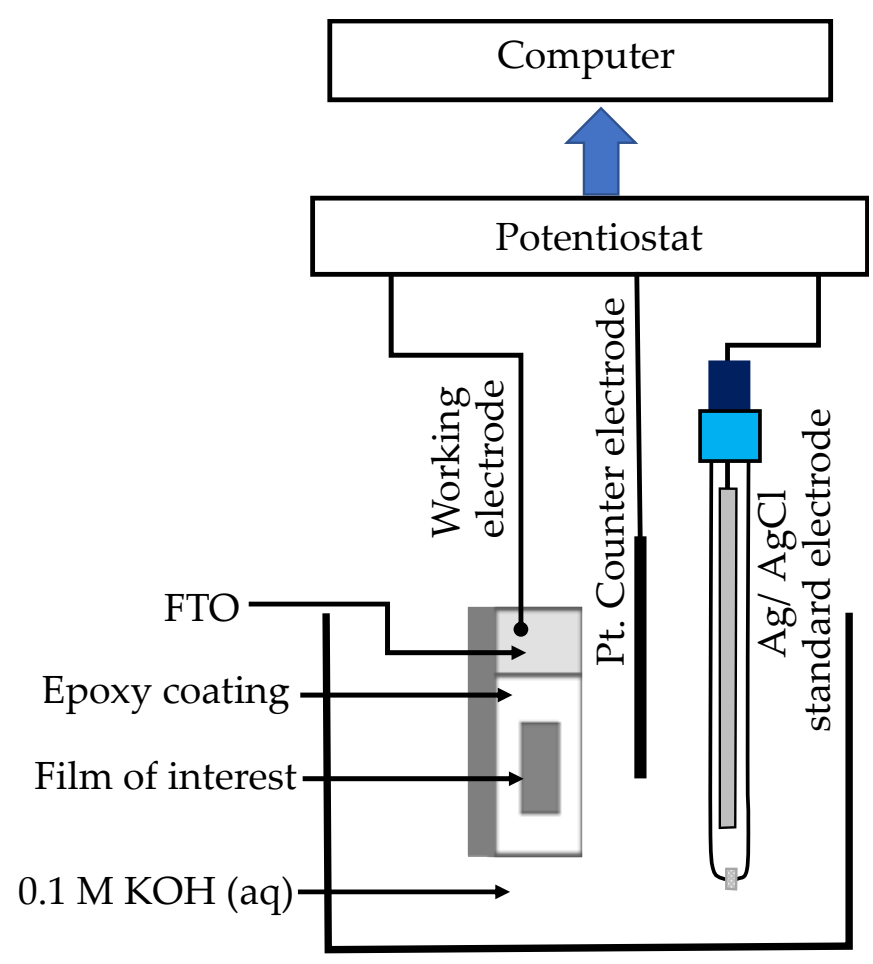

Figure 4.1. Experimental setup for the electrochemical measurements of doped and undoped titania. 


\subsection{Results and Discussion}

\subsubsection{Electrical Conductivity Measurements}

Figure 4.2a shows an SEM image of the nanocrystalline titanium dioxide (NTD) film deposited in the $11 \mu \mathrm{m}$ gap region between the two gold electrodes on the oxidized silicon wafer. This two-terminal device mounted in an optical cryostat was used for solidstate conductivity measurements of the films in the dark or light as illustrated in Figure 4.2b.
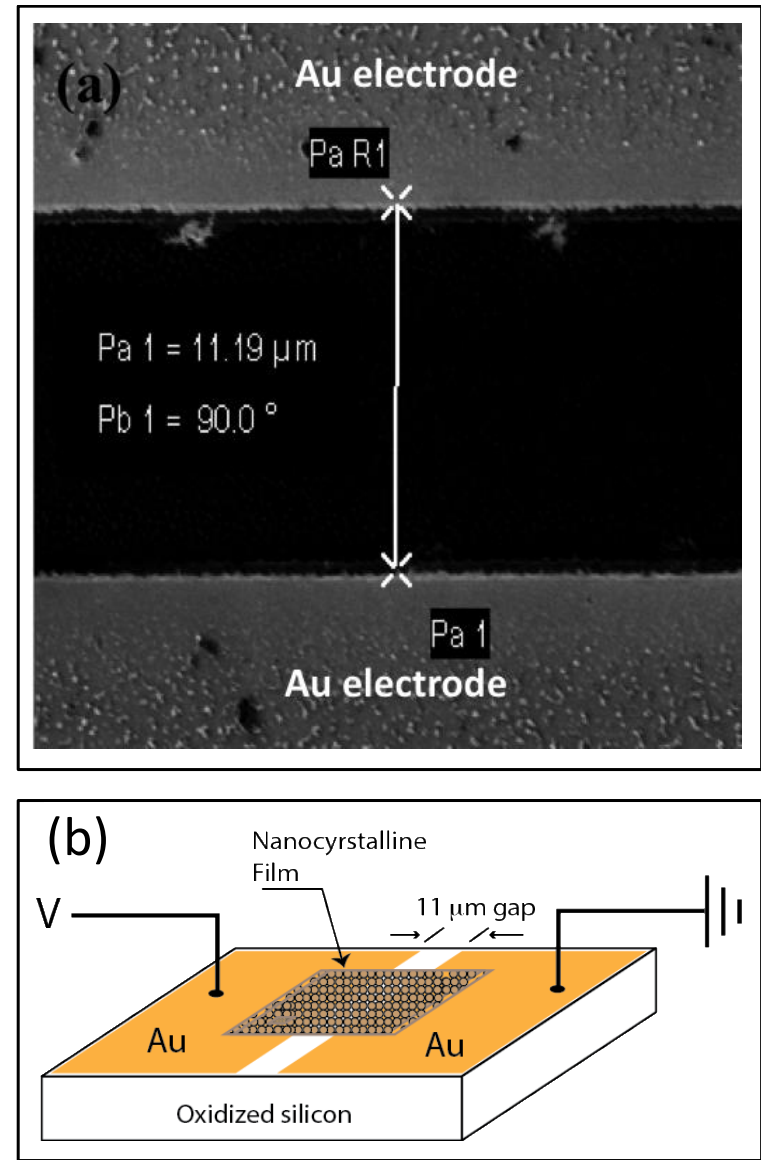

Figure 4.2. (a) SEM image and (b) illustration of the device used for solid-state conductivity measurements. 
Figure 4.3a-c shows the two-terminal current-voltage characteristics of the doped and undoped titania. The currents were measured both in light and dark. The current increases in the presence of light due to the photo excitation of charge carriers from lower energy band states and trap states. ${ }^{138}$ Doping is found to increase the conductance of the titania by a factor of 40-50. In light, the current-voltage characteristics in both doped and undoped films are approximately linear. However, in dark, as shown in Figure 4.3a, the undoped film shows non-linear behavior, with very poor conductance up to a bias of about $0.3 \mathrm{~V}$ and increasing conductance above that. This can be attributed to deep trap states resulting from grain boundaries, ${ }^{172}$ oxygen vacancies, and un-coordinated Ti-atoms. ${ }^{158}$ The trap states increase scattering of mobile charge carriers and introduce a barrier for charge transport due to band-bending at the contact/titania interface. Figure 4.3b-c shows that the conductance near zero bias increases and becomes more ohmic by doping the titania with the REO NPs. Further measurements show that the conductivity of a film of REO NPs only is lower than that of an undoped titania film as shown in Figure 4.4. This shows that the conductance increase of titania with REO doping is not simply due to the introduction of a more highly conductive material into the mesoporous film. 

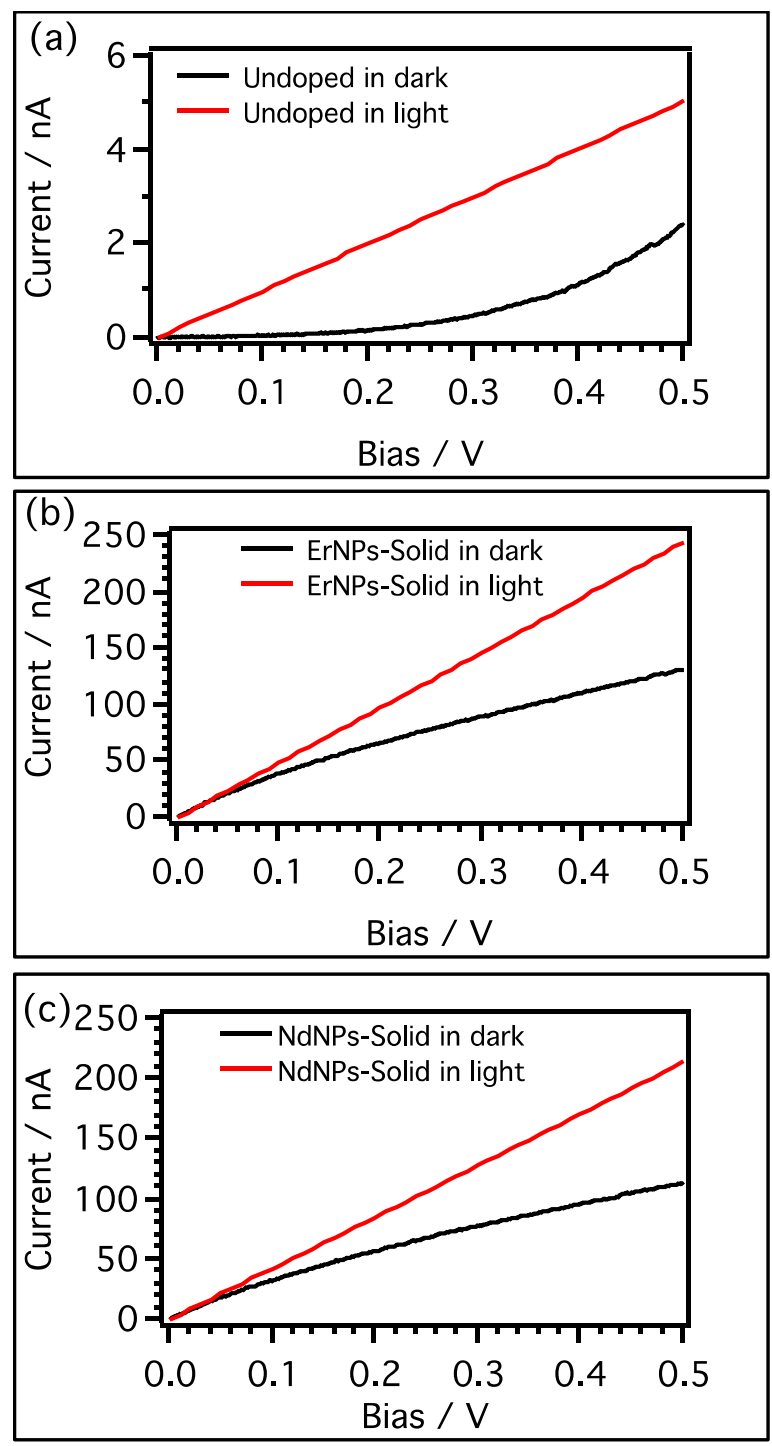

Figure 4.3. Solid-state conductivity measurements of (a) undoped titania, (b) $\mathrm{Er}_{2} \mathrm{O}_{3}$ doped titania, and (c) $\mathrm{Nd}_{2} \mathrm{O}_{3}$-doped titania in dark and light as indicated. 

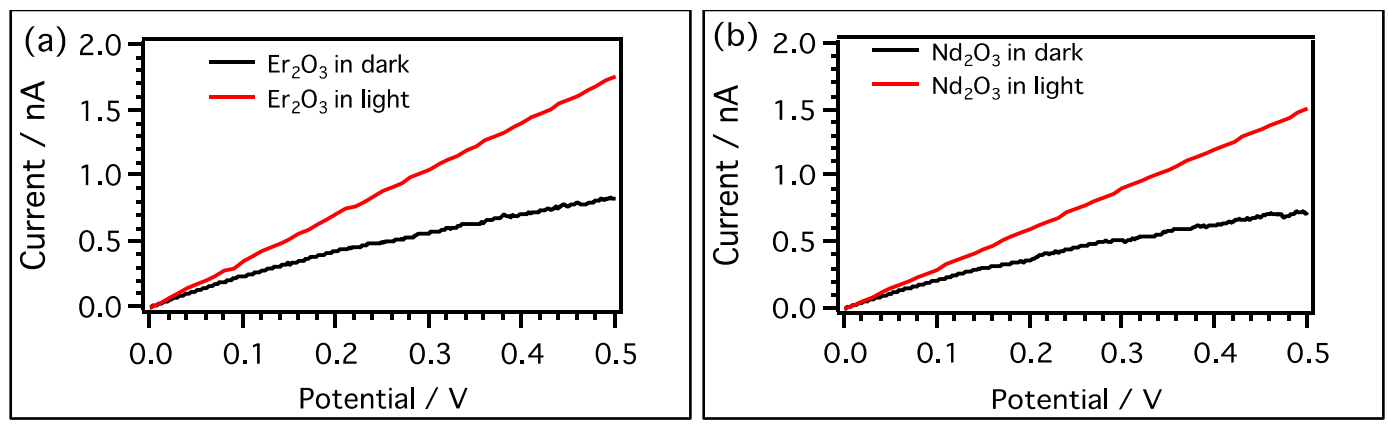

Figure 4.4. Conductivity measurements of films of pure (a) $\mathrm{Er}_{2} \mathrm{O}_{3}$ and (b) $\mathrm{Nd}_{2} \mathrm{O}_{3}$ nanoparticles. The films of REO NPs are less conductive than undoped titania.

\subsubsection{Electrochemical Measurements}

Further investigation of the enhancement in the conductivity of titania due to REOdoping was carried out through a cyclic voltammetric $(\mathrm{CV})$ study of the doped and undoped films using a three-electrode setup and $0.1 \mathrm{M} \mathrm{KOH}$ as the electrolyte. The full CVs of doped and undoped electrodes at various scan rates ranging from 5 to $600 \mathrm{mV} / \mathrm{s}$ are given in Figure 4.5a-c. Figure 4.6a shows the cathodic current only for the undoped film for sweep rates ranging from 100 to $600 \mathrm{mV} / \mathrm{s}$ scanning in the negative direction. A peak is observed at around 0.05 to $0.0 \mathrm{~V}$, which is associated with electron transport to the titania conduction band (CB) and filling of localized surface states deep in the band gap ${ }^{145}$ caused by grain boundary defects ${ }^{160}$ and oxygen vacancies. ${ }^{33}$ The position of the defect peak moves to more negative voltage with increasing sweep rate due to the time it takes to fill the deep trap states. Figure 4.6b-c shows the same CVs for the $\mathrm{Er}_{2} \mathrm{O}_{3}$-doped and $\mathrm{Nd}_{2} \mathrm{O}_{3}$ doped titania samples, respectively. In both cases, the initial peak potential is more negative than the undoped sample and has a larger negative shift in potential with increasing scan rate. 

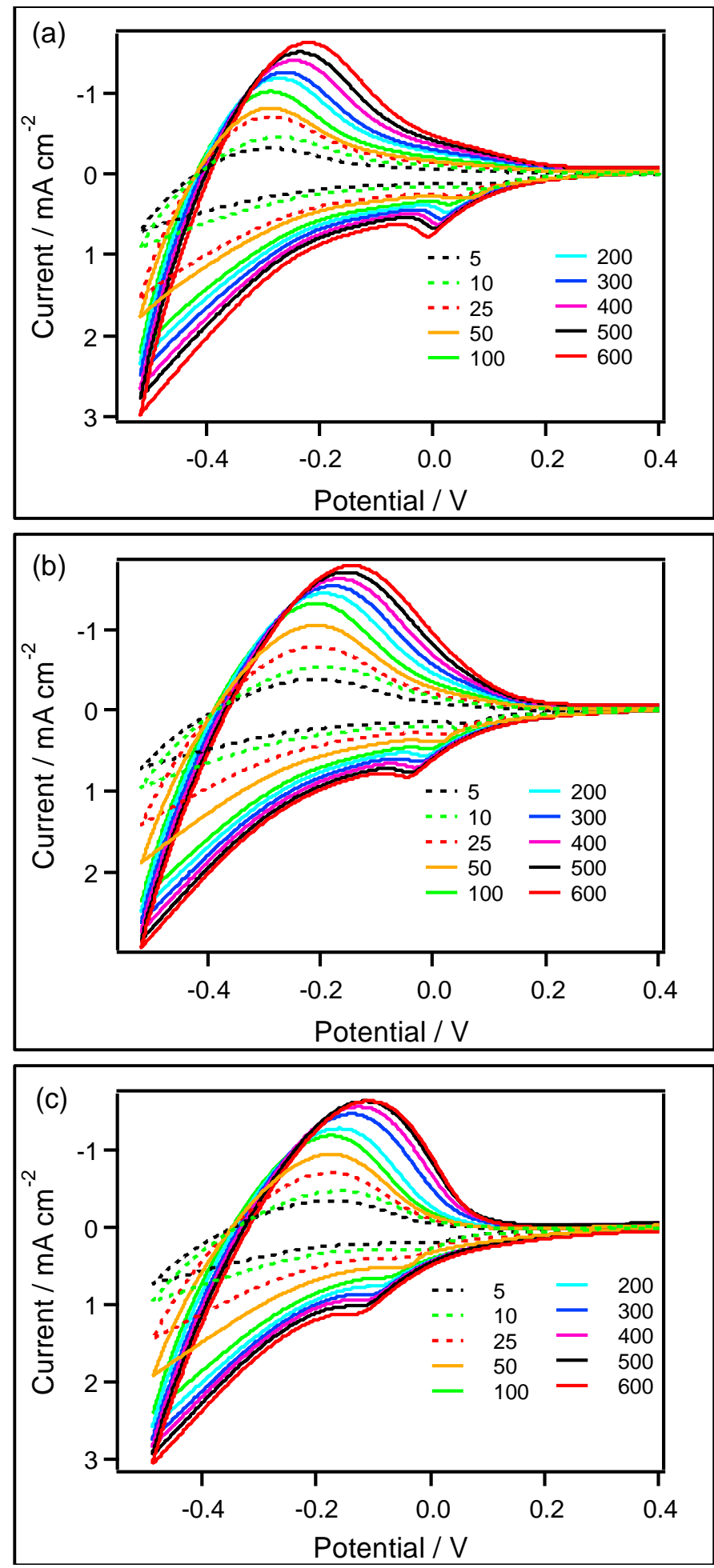

Figure 4.5. Cyclic voltammograms of (a) undoped, (b) ErNPs-Solid and (c) NdNPsSolid at different scan rates as shown in the keys. The scan rates are in $\mathrm{mV} / \mathrm{s}$. 

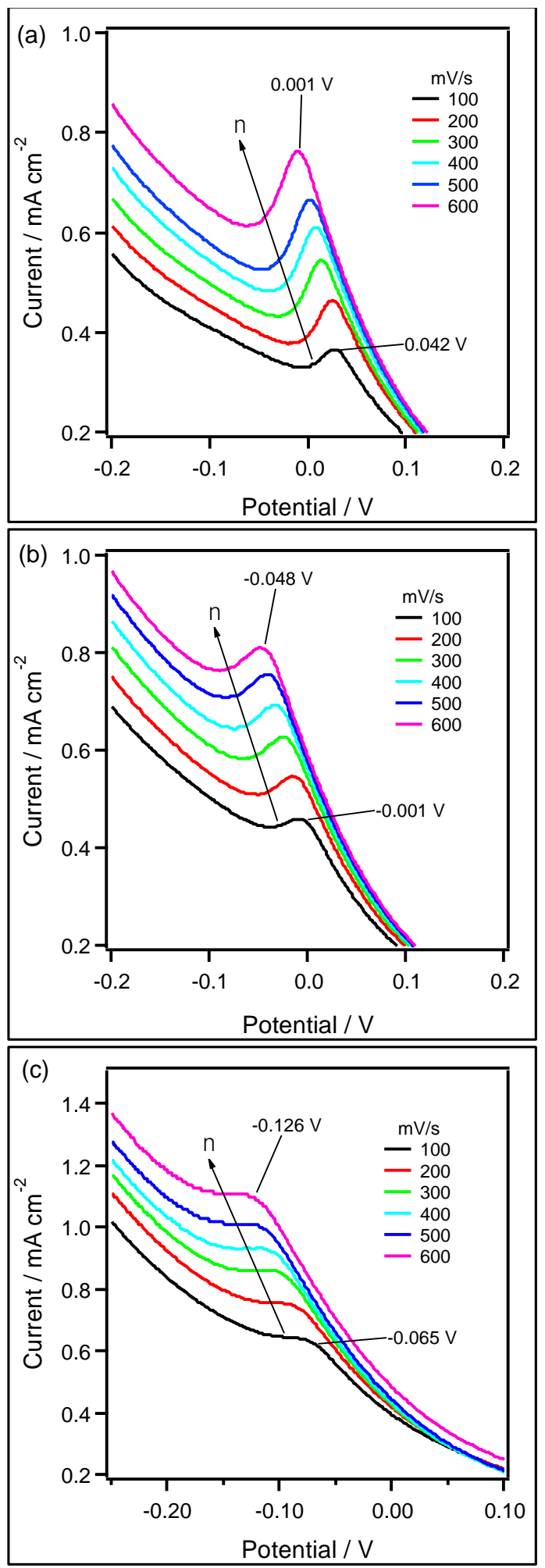

Figure 4.6. Cathodic peak positions of (a) undoped, (b) $\mathrm{Er}_{2} \mathrm{O}_{3}$-doped, and (c) $\mathrm{Nd}_{2} \mathrm{O}_{3}-$ doped titania at different scan rates. 


\subsubsection{Model for Charge Trapping and Detrapping Kinetics}

We used a model of trapping and de-trapping in nanocrystalline titania developed by Bisquert and co-workers ${ }^{158}$ to better understand the influence of REOs on the trapping kinetics.

The change in the probability $f$ that a trap state is occupied as a function of time is given by:

$$
\frac{d f(t)}{d t}=\frac{1}{\tau_{t r}(t)}[1-f]-\frac{1}{\tau_{d t r}} f \ldots
$$

where $1 / \tau_{t r}$ is the trapping rate and $1 / \tau_{d t r}$ is the de-trapping rate. For deep level traps, assuming that the rate of capture is proportional to the number of electrons in the conduction band, the de-trapping rate is assumed to be zero, while the trapping rate is given by:

$$
\frac{1}{\tau_{t r}(t)}=\beta_{n} n_{c}=\frac{1}{\tau_{0}} e^{\left(E_{F n}(t)-E_{F O}\right) / k T} \ldots
$$

where $n_{c}$ is the density of electrons in the conduction band and $\beta_{n}$ is the time constant per unit volume for electron capture.

The time dependence comes about because the Fermi energy changes as a function of time with potential:

$$
-\left(E_{F n}(t)-E_{F 0}\right) / q=V_{0}-v t \ldots
$$

Here, $V_{0}$ is the starting bias, and $v$ is the bias scan rate.

The trapping rate can then be re-written as:

$$
\frac{1}{\tau_{t r}(t)}=1 / \tau_{0} e^{-q\left(V_{0}-v t\right) / k T}=g(t) \ldots
$$


The function $g(t)$ is introduced to make the mathematics easier to follow. Taking the derivative of Eq. (4.4) gives:

$$
\frac{d g(t)}{d t}=\frac{q v}{k T} g(t)=\frac{1}{\tau_{v}} g(t)
$$

where $\tau_{v}=k T / q v$ is introduced. Solving the differential equation (4.1), the occupation probability $f(t)$ should be given as:

$$
f(t)=1-e^{\left(\tau_{v} g(0)-\tau_{v} g(t)\right)} \ldots
$$

Taking the derivative of Eq. (4.5),

$$
\frac{d f(t)}{d t}=\tau_{\nu} e^{\left(\tau_{\nu} g(0)-\tau_{v} g(t)\right)} \frac{d g(t)}{d t}=g(t) e^{\left(\tau_{\nu} g(0)-\tau_{\nu} g(t)\right)}
$$

Re-writing Eq. (4.1) using our definition for $\mathrm{g}(\mathrm{t})$ and taking $1 / \tau_{d t r}=0$ gives:

$$
\frac{d f(t)}{d t}=g(t)(1-f(t)) \ldots
$$

so clearly the function in (4.5) is a solution to the differential equation (4.6).

Finally, the peak position occurs at the potential where there is maximum change in the trap occupation probability, or at the point where $d^{2} f / d t^{2}=0$. Taking the second derivative of the occupation probability gives:

$$
\frac{d^{2} f}{d t^{2}}=e^{\tau_{v} g(0)}\left[-g^{2}(t) e^{-\tau_{v} g(t)}+\frac{g(t)}{\tau_{v}} e^{-\tau_{v} g(t)}\right]
$$

For this to equal zero, $g(t)=1 / \tau_{v}$, or $\tau_{t r}=k T / q v$. Plugging this into Eq. (5.4) then gives:

$$
\frac{q v}{k T}=1 / \tau_{0} e^{-q V_{p e a k} / k T}=>e^{-q V_{p e a k} / k T}=v / v_{0}
$$


The peak potential $\left(\mathrm{V}_{\mathrm{p}}\right)$ corresponding to defects (deep traps) relates to the scan rate $(v)$ by

$$
\mathrm{e}^{-\mathrm{qv} / \mathrm{pT}}=\frac{v}{v_{0}} \ldots
$$

where $\mathrm{q}$ is electronic charge, $\mathrm{k}$ is the Boltzmann's constant, $\mathrm{T}$ is the absolute temperature, $v$ is scan rate, and $v_{0}$ is defined by

$$
v_{0}=\mathrm{kT} / \mathrm{q} \tau_{0} \ldots
$$

where $\tau_{0}$ is the trapping lifetime of free electrons at equilibrium, which is inversely proportional to the time constant per unit volume for electron capture. Using the trapping model described above, $\mathrm{e}^{-\mathrm{qV}_{\mathrm{p}} / \mathrm{kT}} \mathrm{vs} v$ plots for doped and undoped titania are shown in Figure 4.7a. The slopes of the fit lines, proportional to $\tau_{0}$, are a factor of 6 and 85 times larger for $\mathrm{Er}_{2} \mathrm{O}_{3}$-doped and $\mathrm{Nd}_{2} \mathrm{O}_{3}$-doped samples, respectively, compared to undoped samples. This shows that the rate of electron trapping is significantly slower for REOdoped titania.

Rearrangement of equation (4.7) gives:

$$
\mathrm{V}_{\mathrm{p}}=\frac{\mathrm{kT}}{\mathrm{q}} \ln \left(\mathrm{v}_{0}\right)-\frac{\mathrm{kT}}{\mathrm{q}} \ln (\mathrm{v}) \ldots
$$

Equation (4.9) shows that the value of $v_{0}$ can be determined by taking the y-intercept of a plot of $V_{p} v s \ln (v)$. This can then be used to determine the value of $\tau_{0}$. Figure $4.7 b$ shows the variation of $\mathrm{V}_{\mathrm{p}}$ as a function of $\ln (\mathrm{v})$ for doped and undoped samples. Note that the slope is the same for each set of measurements and is given by $\mathrm{kT} / \mathrm{q}=0.026 \mathrm{~V}$ at $300 \mathrm{~K}$. Only the $y$-intercept changes, as predicted by equation (4.9). The calculation of $\tau_{0}$ from Figure 4.7b is illustrated in Table 4.1 while the kinetic parameters extracted from the plots are given in Table 4.2. Compared to undoped titania, the trapping lifetime $\tau_{0}$ increased by a factor of about 6 for $\mathrm{Er}_{2} \mathrm{O}_{3}$-doping and 70 for $\mathrm{Nd}_{2} \mathrm{O}_{3}$-doping. An increase in trapping lifetime is indicative of a decrease in trap states per unit volume of material since fewer 
trap states means that it takes longer on average for an electron in the conduction band to be trapped. This suggests that the REO doping lowers the effective number of deep trap states.
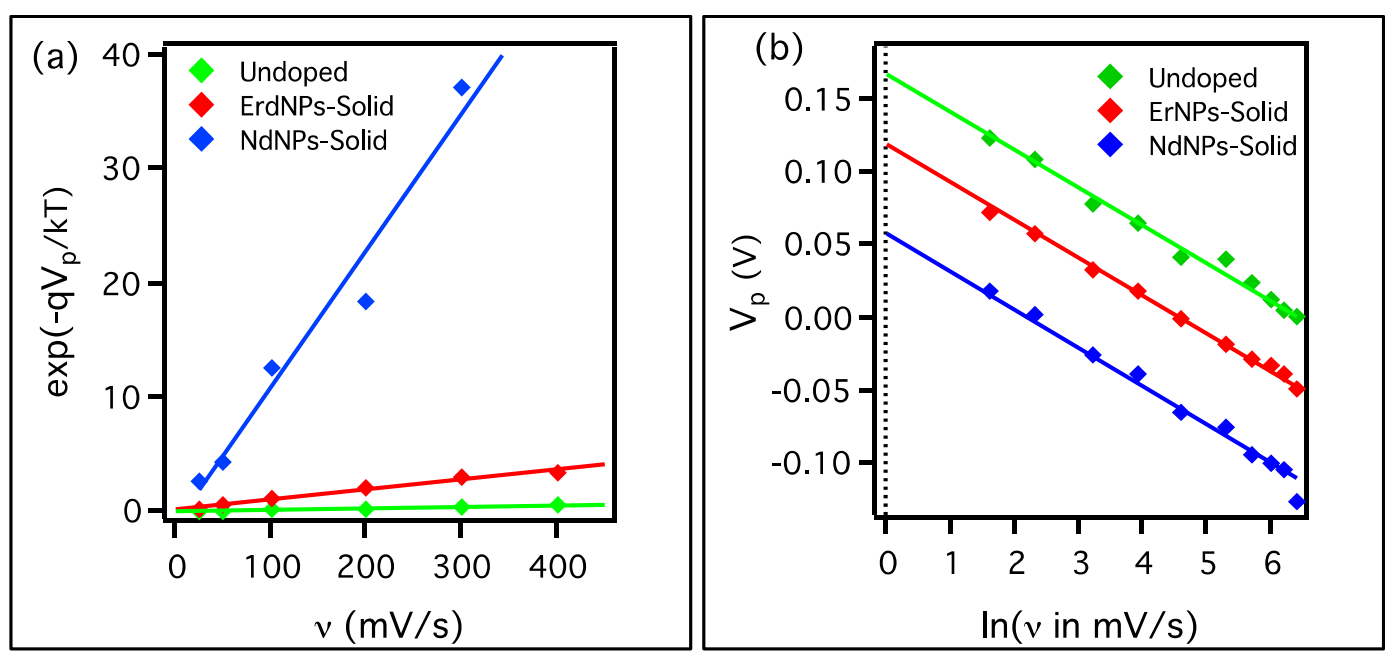

Figure 4.7. (a) Boltzmann type exponential factor calculated at the voltage of the deep trap capacitance peak versus scan rate where dots are experimental values and solid lines are linear fits and (b) plot of $V_{p}$ vs natural logarithm of $v$ in $\mathrm{mV} / \mathrm{s}$, where dots are experimental values and solid lines are the linear fits.

Table 4.1. Calculation of $\tau_{0}$ from Figure $4.7 \mathrm{~b}$

\begin{tabular}{|c|c|c|c|}
\hline Samples & $y-$ intercept $=\frac{k T}{q} \ln \left(v_{0}\right)$ & $v_{0}(\mathrm{mV} / \mathrm{s})$ & $\tau_{0}(\mathrm{~ms})$ \\
\hline Undoped & 0.16756 & 629.3 & 42 \\
\hline ErNPs-Solid & 0.11946 & 98.95 & 263 \\
\hline NdNPs-Solid & 0.057258 & 9.045 & 2874 \\
\hline
\end{tabular}




\subsubsection{Chronoamperometric Measurements}

Chronoamperometric measurements were carried out to determine the influence of the REO-doping on the transient current profile after a potential step. Using the same experimental setup as for the $\mathrm{CV}$ measurements, the potential was stepped to a potential past the defect peak potential and the current was monitored as a function of time. Since the electrolyte used here does not undergo any Faradaic processes during the potential step, the chronoamperometric curves show the charging of the semiconductor/electrolyte interface, revealing the time it takes for all trap states to be filled. Figure 4.8 shows the current versus time plots that result after stepping the potential from +0.4 to $-0.2 \mathrm{~V}$ vs RHE, similar to the procedure for undoped NTD described in the literature. ${ }^{173}$ The charging current drops to the base level in less than $100 \mathrm{~ms}$ for REO-doped titania, while taking about $500 \mathrm{~ms}$ for undoped titania. As described above, there is a higher trap density in the undoped titania relative to the REO doped titania. While individual electron capture is fast, complete charging of the interface takes a longer time, since all of the trap states must be equilibrated.

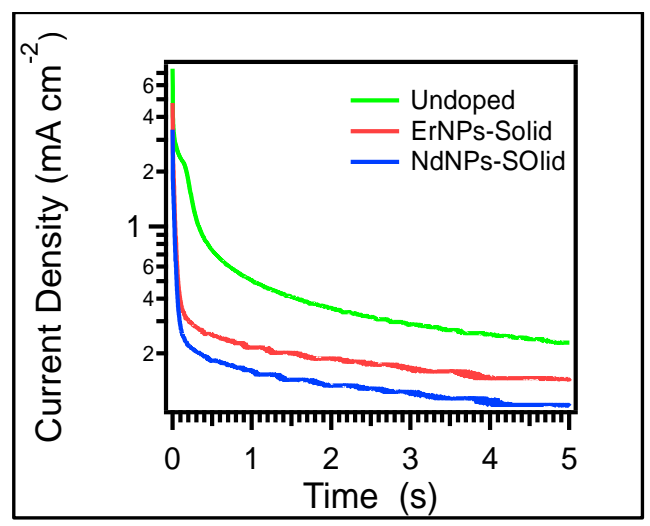

Figure 4.8. Chronoamperometric curves doped and undoped titania. 


\subsubsection{Electrochemical Impedance Measurements}

Next, the influence of REO-doping on the flat-band potential and the carrier density was studied using an electrochemical impedance measurement. As described elsewhere, ${ }^{174-175}$ the space charge capacitance (C) is related to the frequency of the applied ac signal $f$ and the imaginary part of the impedance (Z") by

$$
C=\frac{1}{2 \pi f Z^{\prime}} \ldots(4.10) .
$$

The Mott-Schottky equation relating the change in the space charge capacitance with applied potential is given by ${ }^{175-176}$

$$
\frac{1}{C^{2}}=\frac{2}{\varepsilon \varepsilon_{0} A^{2} e N}\left(V-V_{f b}-\frac{k T}{q}\right) \ldots
$$

where $\mathrm{C}$ is the space charge capacitance, $\mathrm{A}$ the electrode area, $\varepsilon$ the dielectric constant of the semiconductor ( 66 for titanium dioxide), $\varepsilon_{0}$ the permittivity of free space, $q$ the electron charge, $\mathrm{N}$ the space charge density, $V_{f b}$ the flat-band potential and $V$ the applied potential. A plot of $1 / C^{2}$ versus $V$, also known as a Mott-Schottky plot, is linear if $N$ is constant as a function of bias. The slope and x-intercept of the line of fit can be used to calculate $V_{f b}$ and $N$. Figure 4.9a shows the Mott-Schottky plot of doped and undoped titania. The flatband potential is $-0.208 \mathrm{~V}$ vs RHE for undoped titania, which is very close to the value mentioned elsewhere, ${ }^{173}-0.146 \mathrm{~V}$ for $\mathrm{Er}_{2} \mathrm{O}_{3}$-doped and $-0.096 \mathrm{~V}$ for $\mathrm{Nd}_{2} \mathrm{O}_{3}$-doped titania. The space charge densities, $N$, calculated from the slopes of the Mott-Schottky plots are $1.55 \times 10^{22}, 7.47 \times 10^{21}$ and $4.67 \times 10^{21} \mathrm{~cm}^{-2}$ for undoped, $\mathrm{Er}_{2} \mathrm{O}_{3}$-doped and $\mathrm{Nd}_{2} \mathrm{O}_{3}$-doped titania respectively. These values are summarized in Table 4.2. 

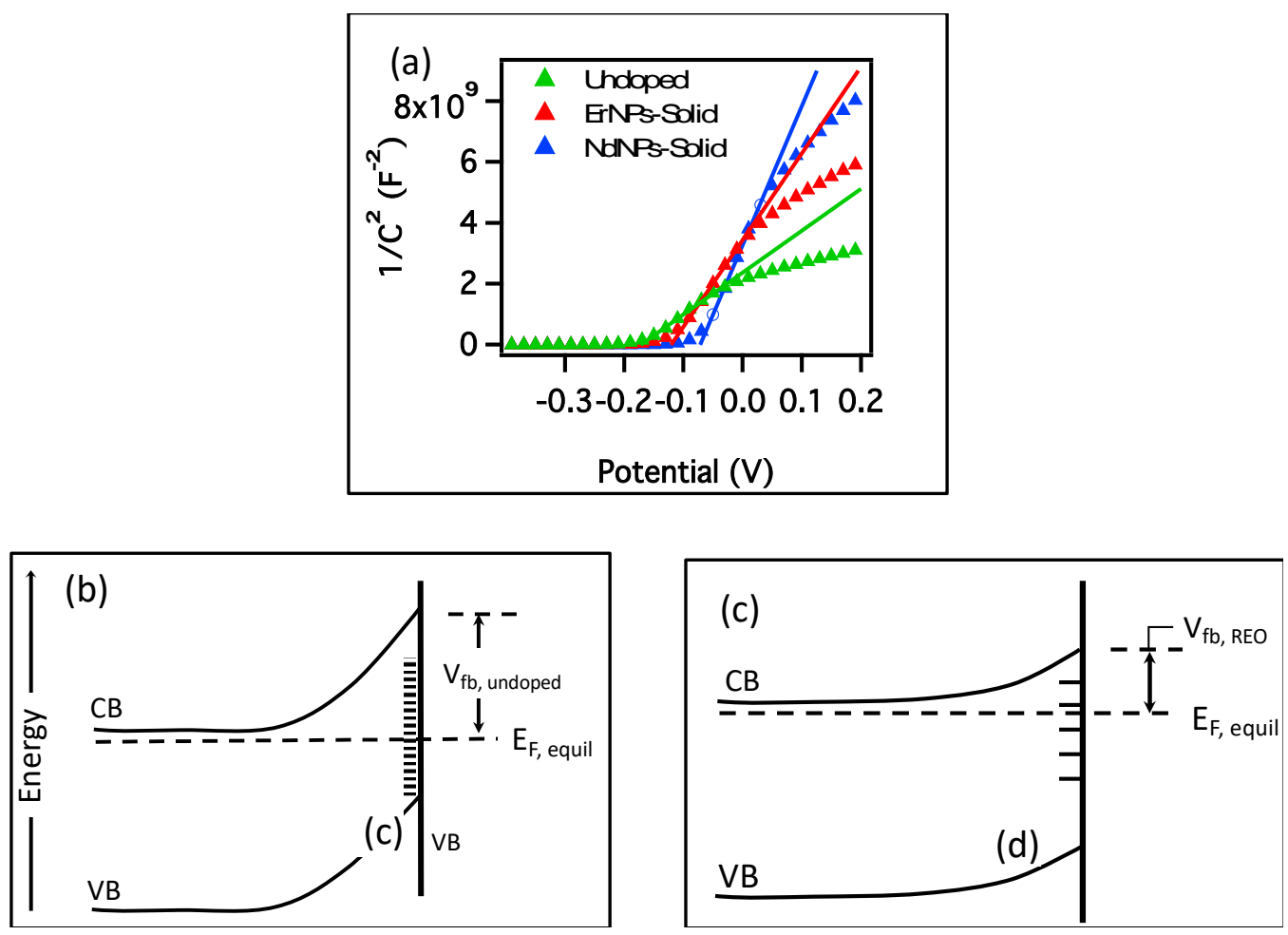

Figure 4.9. (a) Mott-Schottky plots of doped and undoped titania. Illustration of band bending in (b) undoped and (c) REO-doped

Table 4.2. Parameters extracted from Figure 4.7b and 4.9a for doped and undoped titania.

\begin{tabular}{|c|c|c|c|}
\hline Samples & $\tau_{0}(\mathrm{~ms})$ & $V_{f b}(\mathrm{mV}) \mathrm{vs} \mathrm{RHE}$ & $N\left(\mathrm{~cm}^{-2}\right)$ \\
\hline Undoped & 42 & -208 & $1.55 \times 10^{22}$ \\
\hline ErNPs-Solid & 263 & -146 & $7.47 \times 10^{21}$ \\
\hline NdNPs-Solid & 2874 & -96 & $4.67 \times 10^{21}$ \\
\hline
\end{tabular}

The shift in $V_{f b}$ and change in space charge density $N$ can be explained by the difference in trap state concentrations near the titania nanocrystalline surface. ${ }^{177}$ This is shown schematically in Figures $4.9 \mathrm{~b}$ and $4.9 \mathrm{c}$, assuming that the titania is n-type (i.e., the 
Fermi energy lies closer to the conduction band than to the valence band). At the surface, charge from the conduction band fills the lower energy trap states lying in the bandgap. This raises the conduction band as the Fermi energy is pinned in the trap states. The amount of shift, and hence the flat-band voltage $V_{f b}$, will depend on the density of surface traps. ${ }^{178}$ A higher density of trap states will depopulate the conduction band more, so that a larger energy shift will occur, as shown in Figure 4.9a. ${ }^{179}$ Figure 4.9c shows that REO doping neutralizes the surface traps, resulting in a lower flat-band voltage shift relative to the undoped titania. The difference in trap-state concentrations also explains the change in space-charge density. A larger trap concentration means that a change in potential results in a larger change in charge stored at the titania/electrolyte interface in the undoped than in the doped titania. In other words, the capacitance of the undoped titania/electrolyte junction is higher than the doped titania/electrolyte junction due to the high concentration of trap states. This agrees with the results of Figure 4.8, where the total charge transferred to the titania is largest in the undoped sample.

\subsubsection{Mechanism of Trap Neutralization}

The model band-diagram in Figure 4.10 shows a possible mechanism for the trapneutralization in the titania by the $\mathrm{REO}\left(\mathrm{Nd}_{2} \mathrm{O}_{3}\right)$. The theoretical $\mathrm{CB}$ and $\mathrm{VB}$ positions of titania and REO $\left(\mathrm{Nd}_{2} \mathrm{O}_{3}\right)$ were calculated using the procedure described elsewhere ${ }^{180-181}$ from the first ionization potential and the electron affinities of the constituent elements and the band gap energies of $\mathrm{TiO}_{2}$ and $\mathrm{Nd}_{2} \mathrm{O}_{3} .{ }^{182-183}$ The electron affinity for REOs is low ${ }^{184}$ compared to titania, ${ }^{185}$ making it possible that the REO valence band lies higher in energy than the mid-gap states in the titania. It would provide a source of charge to neutralize the trap states. The REO also has a higher energy f-band that could contribute charge, ${ }^{186}$ however, it is not clear whether these states are too tightly bound to the REO atoms to be transferred to the titania. It is interesting that the $\mathrm{Nd}_{2} \mathrm{O}_{3}$ is more effective than the $\mathrm{Er}_{2} \mathrm{O}_{3}$ at neutralizing trap states, although earlier experiments have shown that $\mathrm{Er}_{2} \mathrm{O}_{3}$ doping produces a larger increase in solar cell efficiency in DSSCs than $\mathrm{Nd}_{2} \mathrm{O}_{3}$. ${ }^{170}$ This shows that the decrease in titania impedance shown here is not the only factor involved in the improvement in solar cell efficiency. In fact we also showed that increased surface area accounts for the increase in solar cell efficiency. ${ }^{170}$ 


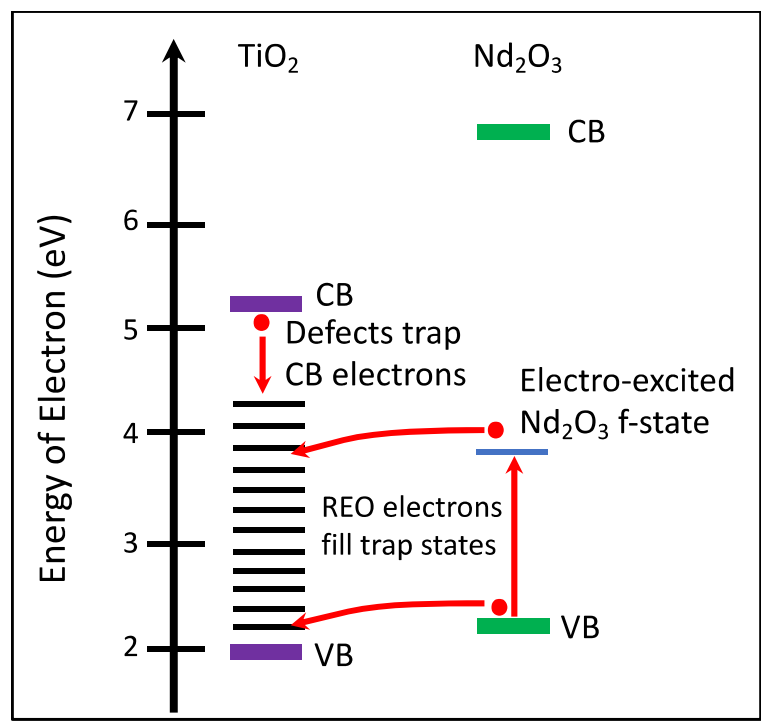

Figure 4.10. Energy diagram of $\mathrm{TiO}_{2}$ and $\mathrm{REO}\left(\mathrm{Nd}_{2} \mathrm{O}_{3}\right)$. The trap states close to the $\mathrm{VB}$ of $\mathrm{TiO}_{2}$, which are responsible for the degradation in the electrical properties are filled by the REO VB or f-electrons, thereby reducing the capture of CB electrons of titania.

\subsection{Conclusions}

NTD films have a high density of trap states in the band gap. These traps can be closer to valence band (deep traps) or conduction band (shallower traps). Doping NTD films with REO nanoparticles reduces the number of deep traps or converts the deep traps to shallower traps from where electrons can easily get de-trapped. Consequently, REOdoping slows the electron trapping rate for conduction electrons, since there are fewer traps available for electron capture. The reduction in trap state density is also evident in the decrease in flat band potential, and reduction in capacitance of the NTD film. The increase in lifetime of the transport carriers provided by the decreased trap density provides an explanation for the reduced electrical impedance of the REO doped film, and also partially explains the improved performance of REO-doped NTD in photocatalytic and solar cell applications. 


\section{CHAPTER V}

\section{AN AC PHOTOCURRENT METHOD FOR CHARACTERIZATION OF THE ELECTRIC POLARIZATION IN HYBRID PEROVSKITES}

\subsection{Introduction}

Organic-inorganic perovskites, also known as hybrid perovskites, have been widely used in many devices, including photovoltaics, ${ }^{72-73}$ photodetectors, ${ }^{74}$ lasers, ${ }^{75}$ and light

emitting diodes. ${ }^{68}$ Their reported advantages include continuous bandgap tuning, ${ }^{70,}$, 187-188 long carrier diffusion lengths, high absorption coefficients, ${ }^{64}$ and low exciton binding energies. ${ }^{189}$ High photovoltaic conversion efficiencies have motivated numerous perovskite solar cell (PSC) development efforts. ${ }^{51,73,190-191}$ A serious hindrance to the large-scale development of PSCs is the hysteresis often observed in the perovskite currentvoltage (I-V) characteristics. It is speculated that the photovoltaic performance and the stability of PSCs are anti-correlated to the amount of hysteresis, with better performance and stability observed in devices with lower hysteresis. ${ }^{192}$ Although the hysteresis is detrimental for PSCs, it is potentially useful for non-volatile memory applications, and a number of reports describe the creation of hybrid perovskite memristors. ${ }^{77-78}$

Despite its importance, the exact mechanism for the I-V hysteresis is still unknown. Various possibilities have been explored, including trapping of positive and negative carriers, and ion migration. ${ }^{93-97}$ These explanations all involve a bias dependent change in the electric polarization of the perovskite that is thought to modify the transport properties. 
This also could be related to the internal electric field that exists in perovskite films, even at zero bias, where an additional bias can alter the field further. Recent experiments have measured an internal potential drop in hybrid perovskites using Kelvin probe force microscopy ${ }^{98}$ and capacitance measurements. ${ }^{99}$ However, the measurements were specific to a particular sample and the relationships between the built-in potential and the applied external field, material quality and photocurrent efficiency were not explored.

Here, we present a unique AC photocurrent method for the electrical characterization of a mixed halide hybrid perovskite (MHHP), namely $\mathrm{CH}_{3} \mathrm{NH}_{3} \mathrm{PbI}_{3-\mathrm{x}} \mathrm{Cl}_{\mathrm{x}}$. Our results reveal that electron-hole pairs generated by electromagnetic radiation interact with the internal electric field, which can be detected through an AC photocurrent measurement similar to the capacitive AC photocurrent measurement described in our previous reports. ${ }^{193-196}$ The AC technique allows charge separation to be measured, which does not appear in the DC photocurrent due to the overly high impedance of the contacts. The technique involves illuminating the sample with a low frequency chopped light source and measuring the AC photocurrent across the sample. The AC photocurrent is non-zero at zero applied bias, showing that there is a built-in force (polarization) driving charge separation. We measure the magnitude of the electric polarization by measuring the magnitude of applied bias required to block the $\mathrm{AC}$ photocurrent. Using this technique, we show how the magnitude and direction of polarization can be modified by applying a poling field. After poling, the electric polarization slowly returns to its initial value. The initial polarization varies with the perovskite grain size and precursor concentrations used during the synthesis. The dependence on grain size can be used to judge perovskite film quality.

\subsection{Experimental Section}

The details of all the experiments for this study are given in Chapter 2. 


\subsection{Results and Discussions}

\subsubsection{Highly Granular Film and its Characterization}

MHHP films of varying grain sizes were deposited on glass slides and characterized by a number of methods. Figure 5.1a shows an optical image of sample HP3D1, where two interdigitated Au contacts can be seen. The average grain size of the film is $345 \pm 75$ $\mu \mathrm{m}$. Figure 5.1d-e shows the XRD spectra of the MHHP films of average grain sizes of $171 \pm 37 \mu \mathrm{m}$ and $29 \pm 5 \mu \mathrm{m}$, respectively shown in Figure 5.2b-c. The XRD spectra contain peaks characteristic of $\mathrm{CH}_{3} \mathrm{NH}_{3} \mathrm{PbI}_{3-\mathrm{x}} \mathrm{Cl}_{\mathrm{x}}$ perovskite films in agreement with results reported in the literature. ${ }^{16,71,148}$ High intensity peaks at $14.2^{0}$ and $28.6^{0}$ correspond to MHHP while peaks at $15.6^{0}, 31.9^{0}$ and $40.5^{\circ}$ are for $\mathrm{CH}_{3} \mathrm{NH}_{3} \mathrm{PbCl}_{3}$, indicating the segregation of ions during the film making procedure as described elsewhere. ${ }^{41}$ XRD spectra of small grain samples are very similar to the large grain sample except that additional peaks are observed at higher angles marked by * 

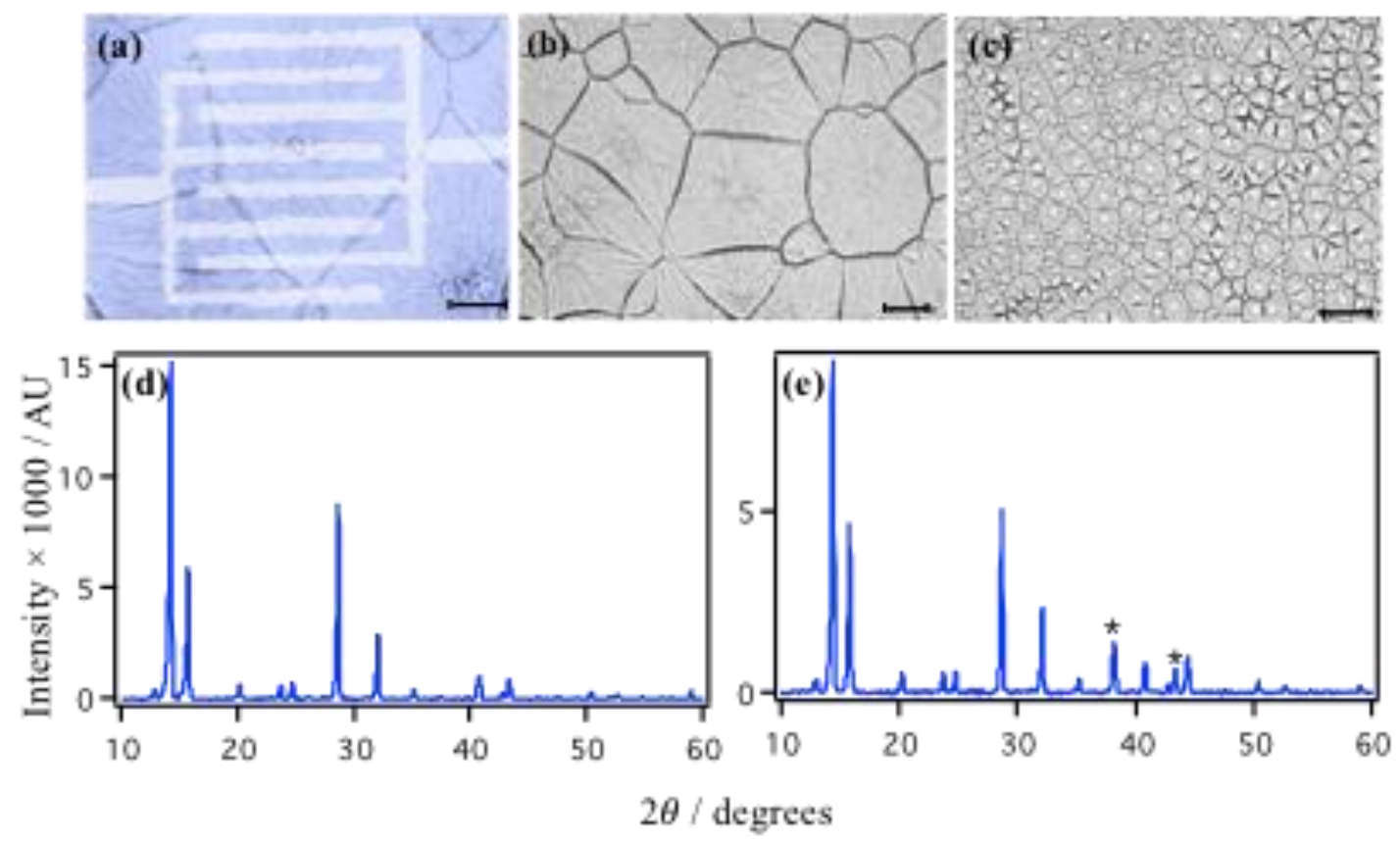

Figure 5.1. (a) Large grain film of MHHP showing a pair of interdigitated Au contacts (device HP3D1, Table 5.1), (b) and (c) optical images of a highly granular and a small grain films with average grain size $171 \pm 37 \mu \mathrm{m}$ and $29 \pm 5 \mu \mathrm{m}$ respectively. The scale bar in Figure a-c is $100 \mu \mathrm{m}$. (d) and (e) XRD spectra of the films shown in Figure b-c.

\subsubsection{Variation of AC Photocurrent with External Electric Field}

Figure 5.2a shows the experimental setup for the ACP measurement (see Chapter 2 for details). Briefly, light from a Tungsten-Halogen source is spectrally resolved using a monochromator and mechanically chopped at a frequency of $13 \mathrm{~Hz}$. The chopped light is then focused on the sample surface in the region of the interdigitated Au contacts. The resulting $\mathrm{AC}$ photocurrent at the chopping frequency is measured between the two $\mathrm{Au}$ contacts using a lock-in amplifier. Figure 5.2b shows an AC photocurrent spectrum of the device HP3D1 measured with zero applied bias (black trace) and an applied bias (the builtin potential, $\mathrm{V}_{\mathrm{bi}}$ ) of $162 \mathrm{mV}$ (red trace). The AC photocurrent spectrum measured with zero applied bias matches the photoabsorbance spectrum (blue trace) for mixed halide hybrid perovskites described in the literature, demonstrating that the signal is due to electron-hole generation in the perovskite film. The beauty of the AC photocurrent 
measurement is that it excludes the absorbance due to substrate. The fact that the AC photocurrent is non-zero with zero applied bias shows that a built-in field must exist in the sample that leads to the separation of the photogenerated electron-hole pairs. In a perovskite solar cell, electron-hole separation is aided by two different contact materials, one electron accepting and another hole accepting. In our case, however, both contact materials are identical, and the contact potentials should cancel out. This is supported by the observation that the open circuit voltage is zero in both light and dark and no DC photocurrent at zero external potential when continuous light is shone on the sample (Figure 5.3a-b). This proves that the AC photocurrent is not due to a potential drop at the contacts. The low AC photocurrent at bias of $162 \mathrm{mV}$ is used to calculate the built-in potential, since the applied bias cancels out the built-in polarization at that potential.

a.

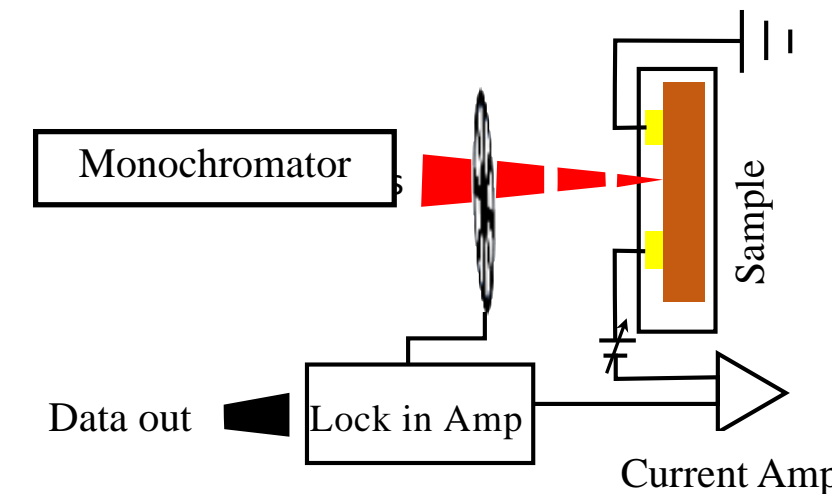

b.

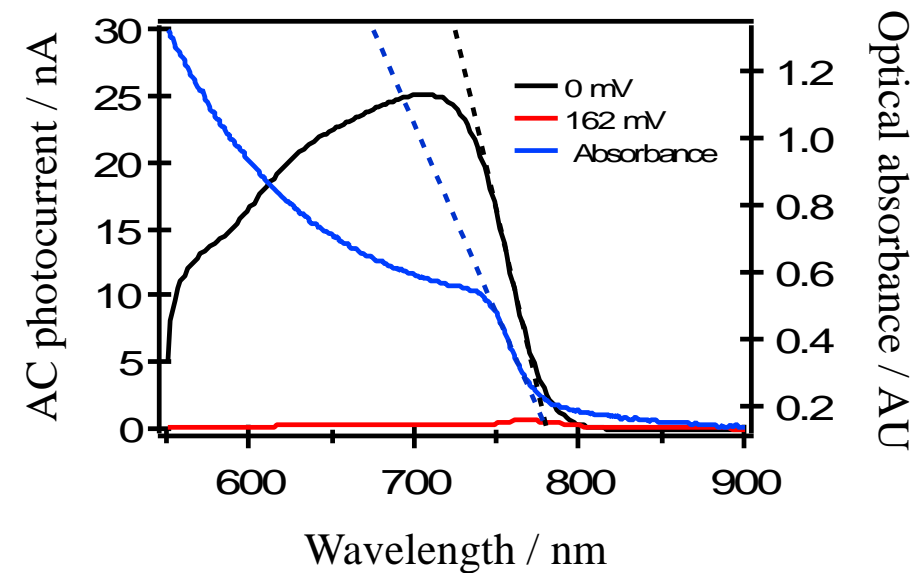

Figure 5.2. (a) Experimental setup for AC photocurrent measurement, (b) AC photocurrent spectrum at no external field $(\mathrm{V}=0 \mathrm{mV})$ and at $\mathrm{V}=\mathrm{V}_{\text {bi }}$. Optical absorbance (blue trace) is shown for comparison. 

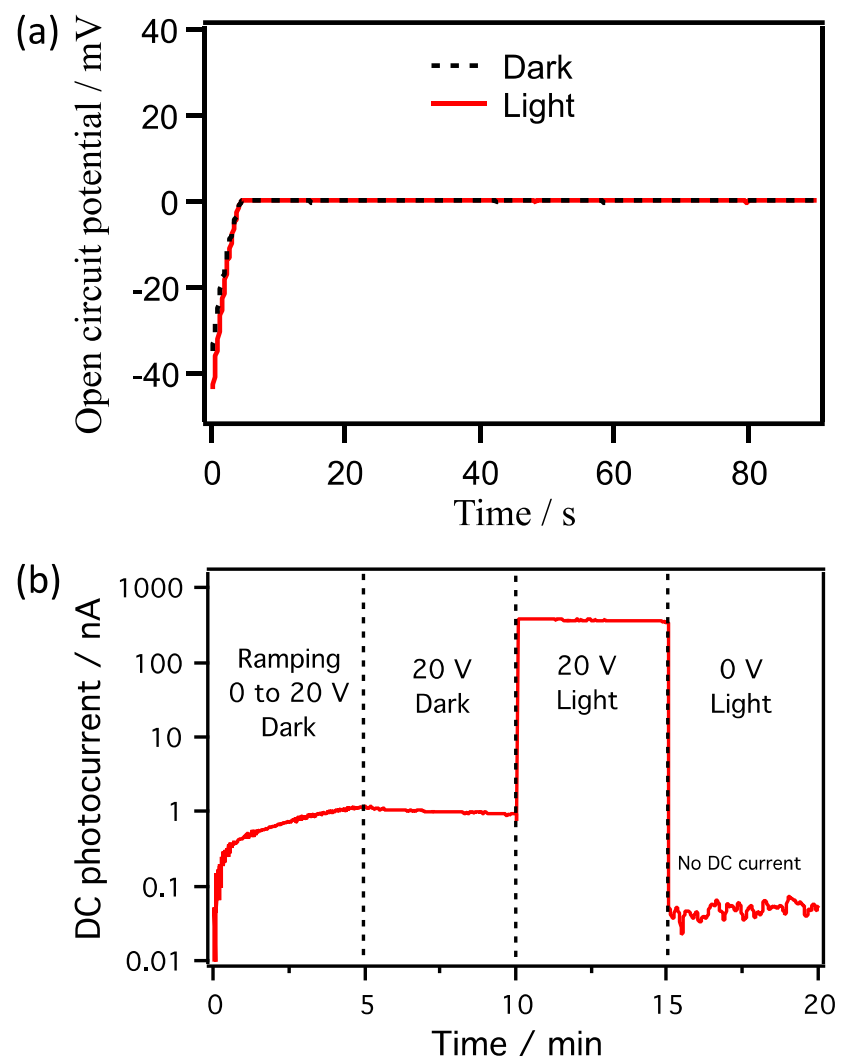

Figure 5.3. (a) Open circuit voltage of the device in the dark and light, and (b) DC photocurrent under various conditions.

\subsubsection{Built-in Potential and Electric Polarization}

Figure 5.4a shows the magnitude (red trace) and phase (blue trace) of the AC photocurrent as a function of applied bias for the device HP3D1. The wavelength of the light was kept fixed at $740 \mathrm{~nm}$, where the photocurrent is maximum. The magnitude of the AC photocurrent decreases as the external bias increases, until it reaches zero at a bias of $162 \mathrm{mV}$. Above this bias value, the magnitude (amplitude) of the AC photocurrent again increases, while the phase of the signal flips by 180 degrees. As described above, the AC photocurrent plot indicates that the magnitude of the built-in potential of the pristine sample $\left(\mathrm{V}_{\mathrm{bi}}\right)$ is $-162 \mathrm{mV}$ based on the zero current at this potential. Further confirmation is given by the results of Figure 5.2b. Here, the AC photocurrent spectrum is measured with an 
applied bias of $162 \mathrm{mV}$ (red trace). The AC photocurrent is zero, independent of the wavelength of the light. Also, we observed that when the contacts are switched, the sign of $\mathrm{V}_{\mathrm{bi}}$ is reversed, indicating that $\mathrm{V}_{\mathrm{bi}}$ is directional.

a.

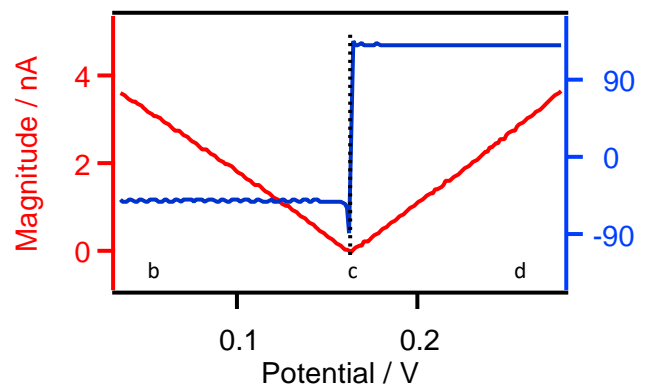

c.

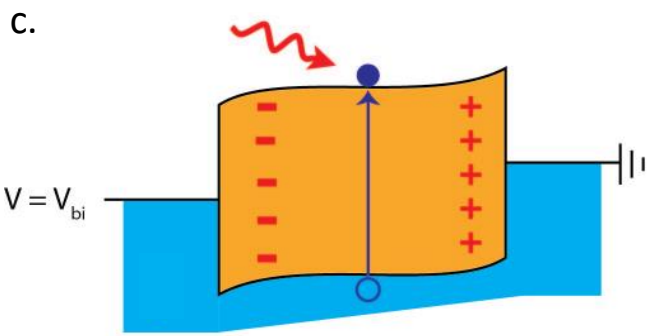

b.

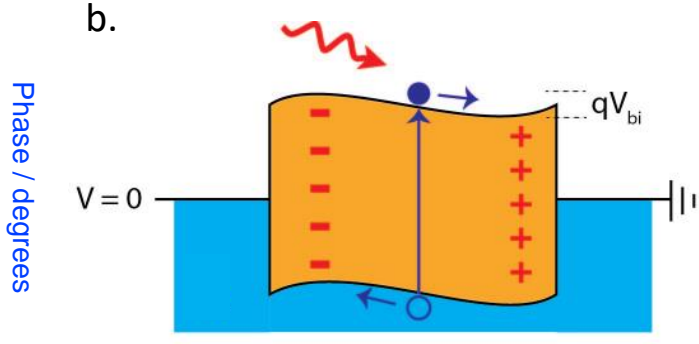

d.

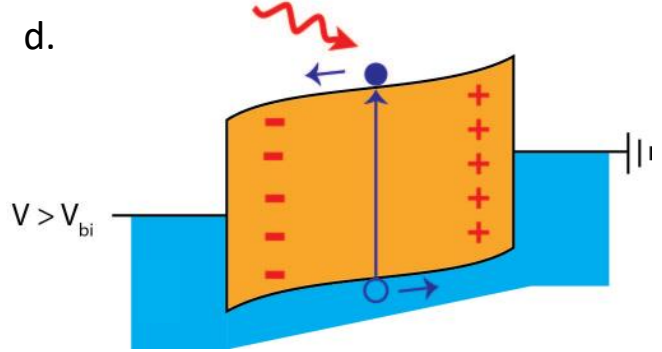

Figure 5.4. (a) AC photocurrent as a function of the external potential, which passes through $\mathrm{V}_{\mathrm{bi}}$, and (b)-(d) band diagrams at external potential less than, equal to and more than $\mathrm{V}_{\mathrm{bi}}$, respectively.

Based on the data, the charge separation and the resulting AC photocurrent must be due to a built-in potential (polarization) with in the perovskite film. Consider the model band-diagram shown in Figure 5.4b. A built-in potential in the perovskite film will separate photogenerated electron-hole pairs, however, if the contact impedance is high, these charges will not be transmitted to the contacts themselves, and hence no DC photocurrent appears. Over time, the charge will simply build-up on the electrode until it compensates for the built-in potential. On the other hand, repeated cycles of carrier generation, separation and recombination by the AC light source does produce a measurable AC photocurrent, provided a potential (built-in or applied) is available to spatially separate the charge carriers. 
The magnitude of the built-in potential can be determined by measuring the impact that an externally applied potential has on the AC photocurrent. Figure 5.4c shows the band-diagram for the sample when an external potential has been applied that is equal in magnitude but opposite in sign to the internal built-in potential, $\mathrm{V}_{\mathrm{bi}}$. The built-in and external potentials balance out so that no charge separation occurs, and the $\mathrm{AC}$ photocurrent goes to zero. Figure 5.4d shows the band-diagram when the external potential is larger than the built-in potential $\left(\mathrm{V}>\mathrm{V}_{\mathrm{bi}}\right)$. Now the charge separation is driven in the opposite direction, so the $\mathrm{AC}$ photocurrent is again non-zero and equal in amplitude from when $\mathrm{V}<\mathrm{V}_{\mathrm{bi}}$, but the phase of the photocurrent is flipped by 180 degrees.

\subsubsection{Grain Size Dependence of Intrinsic Polarization}

The electron transport properties of hybrid perovskites are greatly influenced by the

size of grains. ${ }^{197-198}$ We next considered more carefully the dependence of $\mathrm{P}_{0}$ on the film grain size. We fabricated 4 additional devices using the hot casting procedure having average grain sizes varying between $50 \mu \mathrm{m}$ to $198 \mu \mathrm{m}$, and 1 device fabricated using the drop-coating method, which leads to disordered, poorly-defined grains. Figure 5.5a-e are the optical images of the new films designated as $\operatorname{HP} 3 \mathrm{D}(2-6)$. Following fabrication, we measured the $\mathrm{V}_{\mathrm{bi}}$ for each film using the $\mathrm{AC}$ photocurrent technique described above and calculated $\mathrm{P}_{0}$. Figure 5.5f shows the $\mathrm{P}_{0}$ as a function of average grain size, where the error bars indicate the range of grain sizes in each sample. Values are also given in Table 5.1. Interestingly, we found that $\mathrm{P}_{0}$ does depend on the average grain size and increases from 0 for the drop-cast sample (very small grain) up to $3.58 \mu \mathrm{C} \mathrm{cm}^{-2}$ for the $345 \mu \mathrm{m}$ grain size sample. The $\mathrm{P}_{0}$ appears to saturate as the grain size increases above $200 \mu \mathrm{m}$. A larger $\mathrm{P}_{0}$ can be an indicator of a higher quality film. 

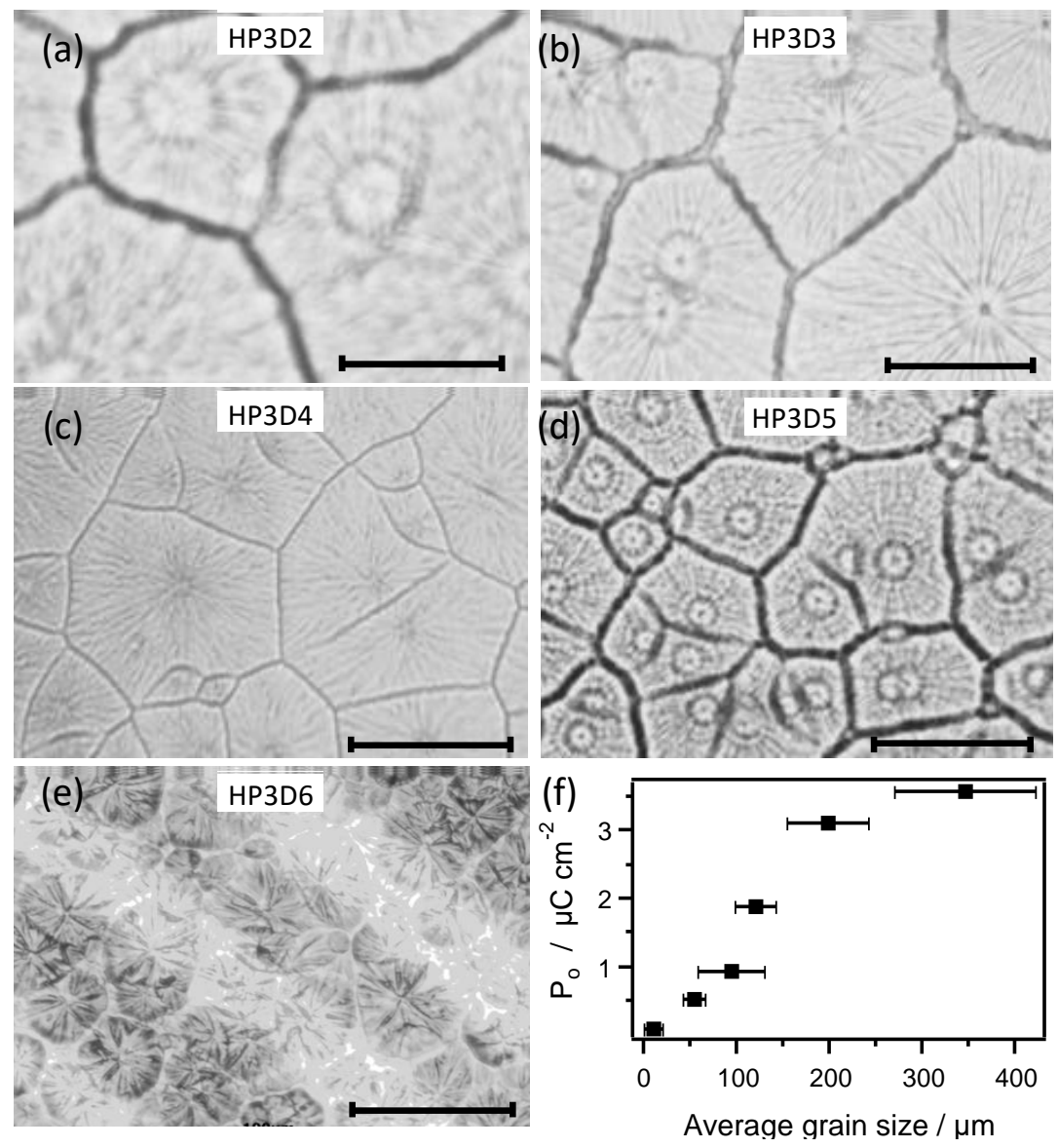

Figure 5.5. (a)-(e) Optical images of devices with varying grain size and (f) variation of zero-bias polarization with grain size. The scale bar is $100 \mu \mathrm{m}$ in each image.

Table 5. 1. Average grain size, measured built-in potential $\left(\mathrm{V}_{\mathrm{bi}}\right)$, and polarization for the different MHHP films used in this study.

\begin{tabular}{|l|c|c|c|}
\hline Name of film & Grain size $(\mu \mathrm{m})$ & $\mathrm{V}_{\mathrm{bi}}(\mathrm{mV})$ & $\begin{array}{l}\text { Polarization } \\
\mu \mathrm{Cm}^{-2}\end{array}$ \\
\hline HP3D1 & $345 \pm 75$ & 162 & 3.58 \\
\hline HP3D2 & $198 \pm 43$ & 141 & 3.12 \\
\hline HP3D3 & $119 \pm 21$ & 86 & 1.90 \\
\hline HP3D4 & $93 \pm 36$ & 42 & 0.929 \\
\hline HP3D5 & $54 \pm 12$ & 24 & 0.531 \\
\hline HP3D6 & - & 4 & 0.088 \\
\hline
\end{tabular}




\subsubsection{Carrier Relaxation after Poling}

We studied the effect of poling on the polarization of various MHHP films. For this study, we poled each of the four devices, HP3D1, HP3D4, HP3D5 and HP3D6 at $5 \mathrm{~V}$ for 1 hour, and carried out AC photocurrent measurements to determine the polarization while the system was relaxing. In all devices under study, there was enhancement in polarization after poling. Figure 5.6a shows the sequence of poling experiments. For the device HP3D1, with the largest grain size, we observed that the polarization increases from $\mathrm{P}_{0}=3.58 \mu \mathrm{C}$ $\mathrm{cm}^{-2}$ to $\mathrm{P}_{\mathrm{v}}=19.2 \mu \mathrm{C} \mathrm{cm}^{-2}$ after poling at $5 \mathrm{~V}$ for $1 \mathrm{~h}$, and returned to $9.87 \mu \mathrm{C} \mathrm{cm}^{-2}$ after 1 $\mathrm{h}$ of relaxation. We believe that poling the device with positive potential caused slow diffusion of the negative ions within the perovskite film towards the positive electrode

while the positive ions within the film diffuse towards the negative electrode. ${ }^{83,199-200}$ This increased charge separation of ionic species increases the polarization and creates an extra built-in electric field in the film, which requires a larger external potential to counterbalance. As soon as the electric field is switched off, the polarized ionic species revert back to their original position, thereby decreasing the field developed in the film. This accounts for the decrease in polarization while the system was relaxing. Figures 5.6b$\mathrm{d}$ show the relaxation of polarization $\left(\mathrm{P}_{\mathrm{v}}\right)$ as a function of logarithm of time in minutes. For the device HP3D1 with very large grain size, $\mathrm{P}_{\mathrm{v}}$ decays linearly with $\log$ (time), while for the device with randomly oriented small grains (or small grain devices), there is a slower decay in $\mathrm{P}_{\mathrm{v}}$. The initial effect of poling persists for a longer time for smaller grain size than for larger grain size films. We speculate that slow initial decay of $\mathrm{P}_{\mathrm{v}}$ can be caused by strong trapping of charge carriers at the defects of grain boundaries. It is very interesting to note that the device reverted back to the initial polarization $\left(\mathrm{P}_{0}\right)$ under inert condition after a long enough time, indicating that the poling effect at $5 \mathrm{~V}$ is slow and reversible in nature and not detrimental to the device performance. 
(a)

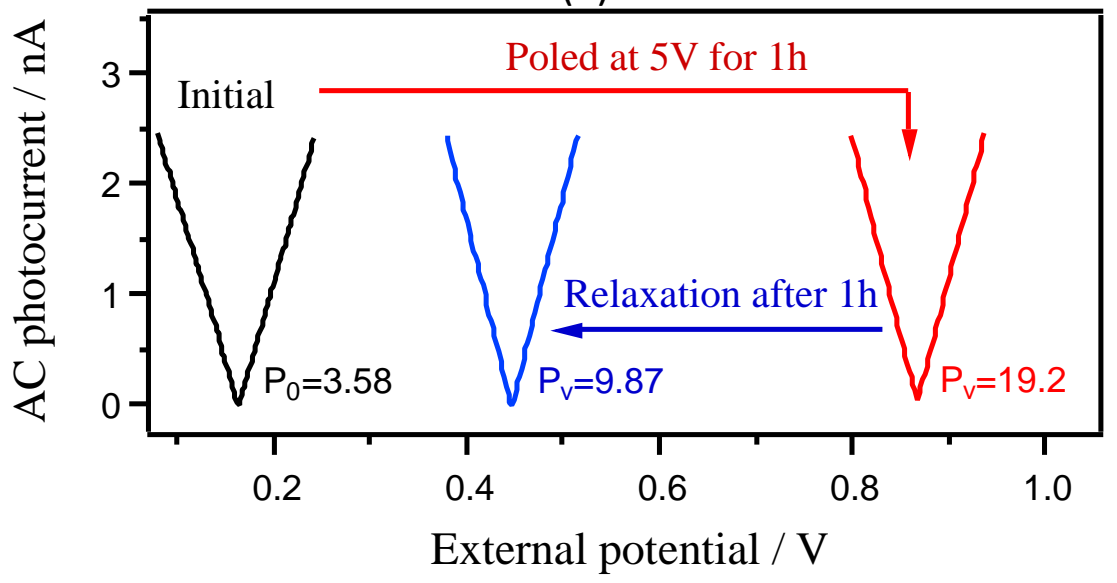

(b)

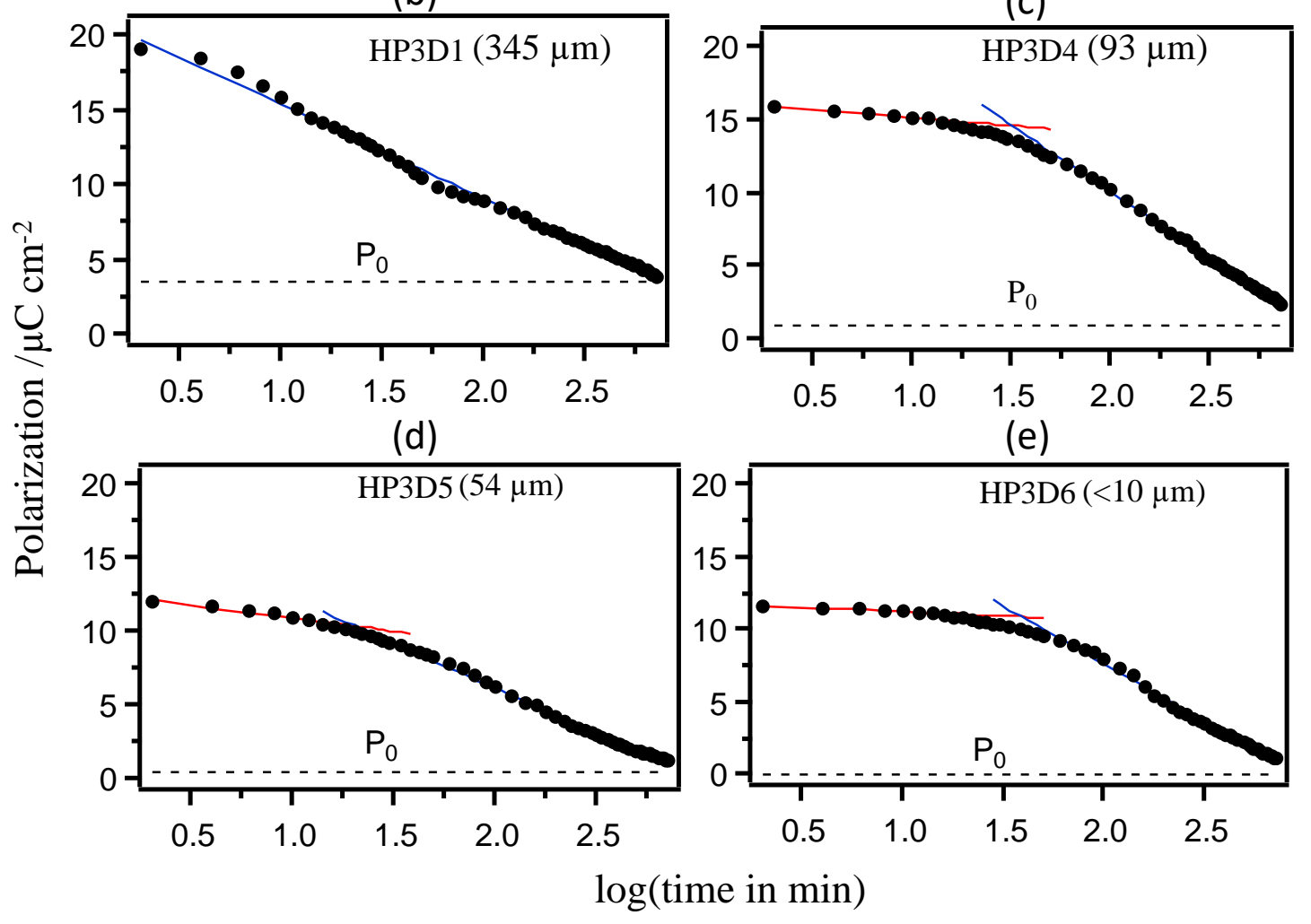

Figure 5.6. (a) Shift of polarization after poling the device HP3D1 at $5 \mathrm{~V}$ for $1 \mathrm{~h}$ and then relaxation after $1 \mathrm{~h}$. The unit of polarization is $\mu \mathrm{C} \mathrm{cm}^{-2}$. (b)-(e) time dependence of polarization of different devices after poling at $5 \mathrm{~V}$ for $1 \mathrm{~h}$. The dashed horizontal line is the initial polarization. 


\subsubsection{Polarization-Electric Field Loops in Perovskite Films}

The AC photocurrent technique is used to measure the polarization versus electric field of the MHHP films. We characterized two different films having average grain sizes of $28 \pm 4 \mu \mathrm{m}$ and $104 \pm 17 \mu \mathrm{m}$ (see Figure 5.7a-b). Measurements were made by stepping the external potential in $0.5 \mathrm{~V}$ increments with a 5 minute wait time between steps. $\mathrm{V}_{\mathrm{bi}}$ was then quickly measured as described in Chapter 2. Figure 5.7c-d shows the resulting polarization $\left(\mathrm{P}_{\mathrm{v}}\right)$ vs applied electric field $(\mathrm{E})$. The polarization of the pristine sample $\left(\mathrm{P}_{0}\right)$ is also shown for comparison. Hysteresis loops are observed in the $\mathrm{P}_{\mathrm{v}}-\mathrm{E}$ curves for both large and small grain samples with a loop width of approximately $2 \mathrm{kV} / \mathrm{cm}$. There is a clear difference in the $\mathrm{P}_{\mathrm{v}}$-E loops for small grain and large grain samples. The small grain sample has $\mathrm{P}_{0}=0$ and the $\mathrm{P}_{\mathrm{v}}-\mathrm{E}$ loop is symmetric around the origin. The large grain sample has $\mathrm{P}_{0}=1.33 \mu \mathrm{C} / \mathrm{cm}^{2}$ and the $\mathrm{P}_{\mathrm{v}}-\mathrm{E}$ loop is centered at about $(0.25,1)$. Also, the polarization is larger at positive electric fields than at negative electric fields because of the internal field of the pristine film, which is added to the field developed during poling. 

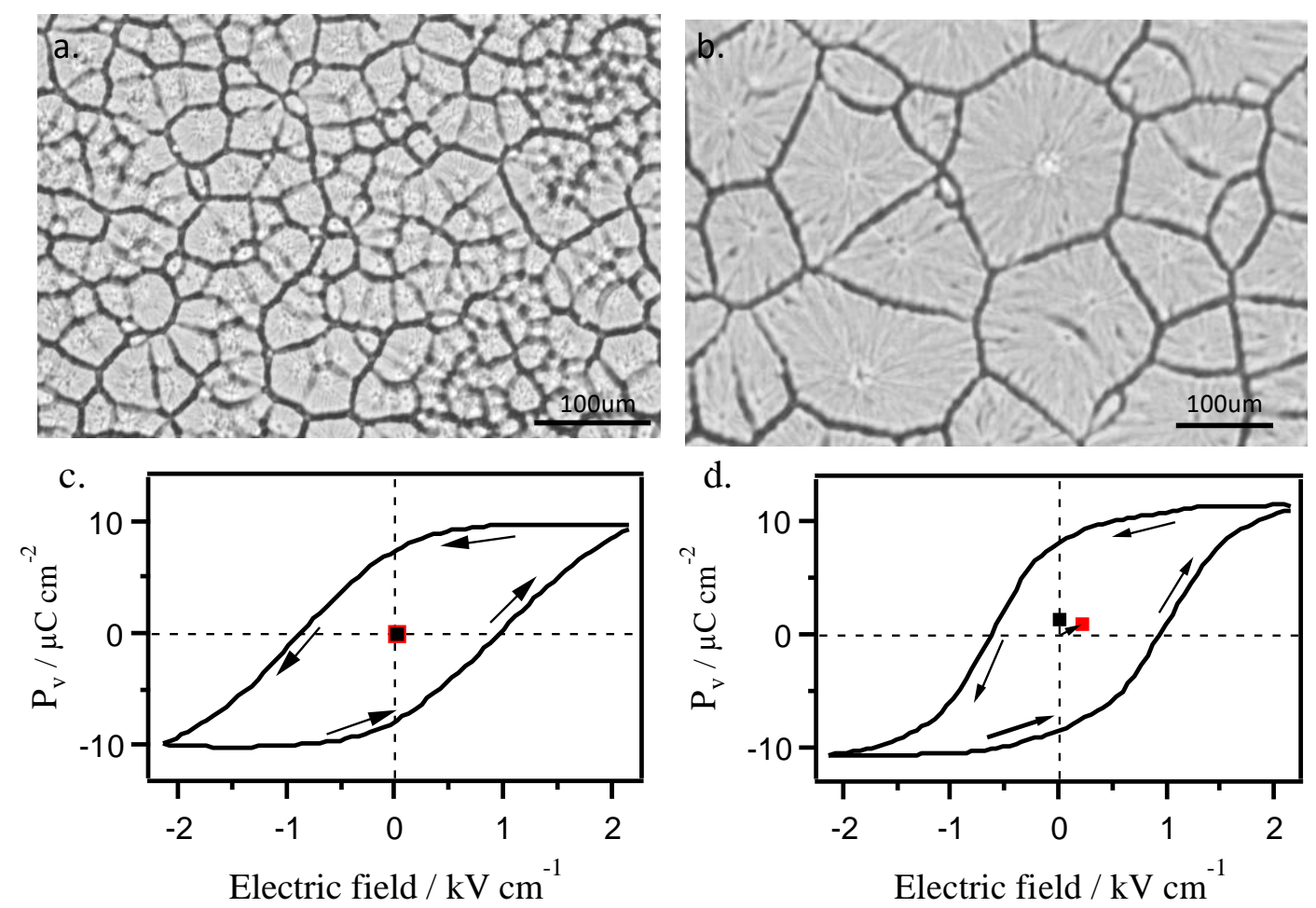

Figure 5.7. Optical images of a small grain film (a) and a large grain film (b) of MHHP, and the corresponding polarization curves (c-d). The black square refers to the polarization of the pristine sample $\left(\mathrm{P}_{0}\right)$ while the red square is an approximate point of symmetry.

\subsubsection{Origin of Intrinsic Polarization and Effect of Ionic Concentration}

Reports $^{66,83,201-204}$ reveal that mismatch between the ionic radii of the different species, vacancy mediated ionic displacements inside the octahedra of crystal and all of the structural distortions in MHHPs lower the centrosymmetry, leading to polar or anti-polar unit-cells. Anti-polar units cancel the effect of one another while polar units add up, resulting in a local intrinsic polarization. Grain boundaries with their structural defects screen the effect of intrinsic polarization, resulting in smaller $\mathrm{P}_{0}$ as shown in Figure 5.5f.

In addition to the lattice distortions caused by photoexcitation, thermal energy, an external electric field, and the composition of the perovskite, ${ }^{205}$ ionic polarization should be considered as a factor that can contribute to the electric polarization. To test the 
hypothesis that ion polarization leads to the built-in potential, films with excess $\mathrm{Pb}^{2+}$ and $\mathrm{I}^{-}$ions relative to $\mathrm{MA}^{+}$were compared to those made stoichiometrically. We prepared films with $\mathrm{PbI}_{2}$ :MACl molar ratios of 1:1, 1.1:1 and 1.2:1 and determined the resulting $\mathrm{P}_{0}$. As shown in Figure 5.8, there is little effect of extra ions on the $\mathrm{P}_{0}$ of small grain size films. This can be attributed to grain boundaries screening the built-in potential, hindering the separation of charge carriers and promoting charge trapping at the defects. For large grain size films $(>100 \mu \mathrm{m})$, there is considerable $\mathrm{P}_{0}$, which can be attributed to the formation of polar domains and reduced screening effect of grain boundaries. These ions/defects diffuse differently in response to an electric field, which can lead to hysteresis in the I-V curves. Reducing the number of grain boundaries can help to reduce the screening effect on $\mathrm{P}_{0}$ and minimize hysteresis in I-V curves for improved solar cell efficiency.

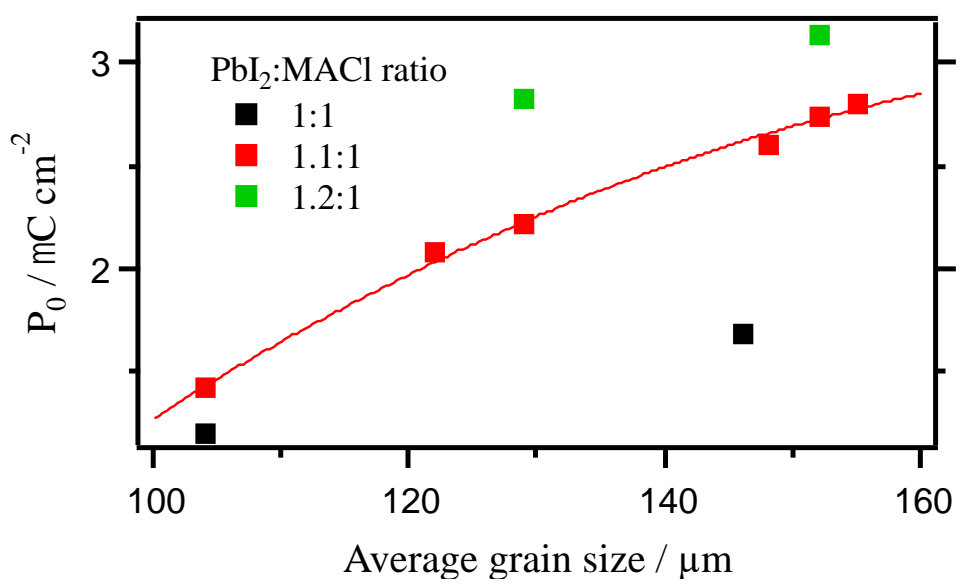

Figure 5.8. Dependence of the electric polarization on the concentration of $\mathrm{PbI}_{2}$.

\subsection{Conclusions}

We used the AC photocurrent method for the electrical characterization of a mixed halide hybrid perovskite of chloride and iodide. The magnitude of the AC photocurrent increases or decreases with external potential and is minimum at $\mathrm{V}_{\text {bi. }}$. The phase of the $A C$ current flips by 180 degrees when the external potential passes through $\mathrm{V}_{\mathrm{bi}}$, indicating that the direction of the current is reversed. The $\mathrm{P}_{0}$ of pristine sample increases with increase 
of the grain size and can be used to check the quality of the film, where a larger $\mathrm{P}_{0}$ means a higher quality film with larger grain sizes. Poling the device above the built-in potential further polarizes the oppositely charged ions and vacancies, and these carriers revert back to their original state when the poling is removed. When the device is relaxed, the detrapping of photogenerated charge carriers takes place. In devices with larger grains, the $\mathrm{V}_{\mathrm{bi}}$ helps in de-trapping the carriers, while in devices with smaller grains, the trapped carriers remain in defect states for longer time because of the screening affect.

As this study was carried out purely with perovskite films only, the influence of interfacial properties ${ }^{206}$ should also be considered when describing the hysteresis in complete PSCs. 


\section{CHAPTER VI}

\section{EFFECT OF LIGHT SOAKING IN ARGON ON MIXED HALIDE HYBRID PEROVSKITE: ENHANCED ELECTRIC POLARIZATION AND IMPROVED PHOTOVOLTAIC PERFORMANCE}

\subsection{Introduction}

Hybrid perovskites have fascinating properties like continuous tuning of the bandgap, ${ }^{70,187-188}$ long carrier diffusion length for photogenerated charge carriers, ${ }^{207-208}$ high absorption coefficient, ${ }^{64}$ nonexcitonic nature/small exciton binding energy, ${ }^{189}$ and switchable photovoltaic effects ${ }^{209}$ for their applications in photovoltaics, ${ }^{72-73}$ photodetectors, ${ }^{74}$ lasing, ${ }^{75-76}$ light emitting diodes, ${ }^{68}$ and memristors. ${ }^{209}$ In just a few years, the photoconversion efficiency (PCE) of perovskite solar cells has surpassed 25\%. ${ }^{73}, 92,210$ Putting aside the toxic nature of lead, ${ }^{88-89}$ the structural, thermal, chemical and photostability are the chief challenges in the large scale implementation of perovskite solar cells (PSCs). ${ }^{90-91}$ Progress has been made for the improvement in stability through the management of the organic cations, inorganic cations and halide ions used in the material. ${ }^{71}$, 73, 90, 92 Encapsulation of PSCs in an inert environment helps to prevent oxidation of the material even under heat and light. There are a number of reports on the effect of light on the crystals and thin films of halide hybrid perovskites. ${ }^{211-214}$ Prolonged illumination of mixed halide hybrid perovskite (MHHP) thin films results in phase segregation, which 
positively affects the optoelectronic properties. ${ }^{215-217}$ In one of the reports by Zhao et al., ${ }^{212}$ it is shown that purposeful light soaking can decrease the surface accumulation of photogenerated charge carriers at the electrode interface, which can neutralize interfacial defects, consequently increasing $\mathrm{V}_{\mathrm{OC}}$. The result of light soaking strongly depends upon the composition of the perovskite. The single halide cubic phase bromide and tetragonal iodide hybrid perovskites showed weak light soaking effects while mixed halide crystalline perovskites showed strong light soaking effects, which is attributed to the trapping of photogenerated charge carriers at surface defect sites, thereby making the defects nonactive. ${ }^{214}$ In a report, ${ }^{213}$ it is shown that long light soaking time helps in curing (reducing) interface trap-assisted recombination. The report shows that light soaking does not change the resistance and the capacitance of the bulk HP layer, however, the chemical capacitance $\left(c_{\mu}\right)$ and energy barrier at the HP/contact interface are reduced, leading to interface trapassisted recombination. Several reports describe photovoltaic improvement of perovskite solar cells (PSC) by light soaking as determined by increased photovoltaic conversion efficiency, reduced hysteresis, and long-term stability. This is the ultimate goal of the working PSC.

The overall photovoltaic performance of a PSC depends on how efficiently the photogenerated charge carriers migrate towards the respective electrodes, electrons towards cathode and holes towards anode. The separation of these excitons is very crucial to reduce the recombination, which can help to get higher photocurrent and photovoltage leading to higher conversion efficiency. In addition to the hole transport and electron transport layers, the intrinsic electric polarization of the perovskite film can help in the separation of excitons, the role of the intrinsic electric polarization for the perovskite-based devices has not been explored. It has been reported that the photoconversion efficiency and stability of PSCs can be enhanced by encapsulating the device in argon gas. ${ }^{210,218}$ The stability improvement is intuitive, but the reason for improved conversion efficiency is not clear. In this report, we describe systematic studies on the influence of gas environment on the electric polarization of MHHP thin films using a novel technique, the AC photocurrent measurement. We show that the background environment plays a significant role for the electric polarization of MHHP films. We herein report that MHHP thin films adsorb Ar 
gas in the presence of light. We measured the photocurrent of MHHP thin films from the visible to near IR region under different environments, including air, oxygen and different inert gases. Interestingly, only the combined effect of solar light and Ar enhanced the photocurrent significantly. Ar plays a significant role in polarizing the photogenerated charge carriers, which increases the electric polarization and deactivate the defect sites in the film to get higher photovoltage and photocurrent. We tested this observation with thin films in an actual PSC of planar p-i-n configuration using MHHP light absorber. The photovoltaic improvement of MHHP solar cells is related not only to the light soaking effect, but also on the environment to which the cell is exposed. We found that the combined effect of light and Ar gas changed the photocurrent density, open circuit potential and conversion efficiency of MHHPSCs drastically. The photovoltaic improvements of Ar gas-encapsulated PSCs are due to the combined effect of both Ar and light soaking.

\subsection{Experimental Section}

The details of the experiments performed for this study are given in Chapter 2.

\subsection{Results}

\subsubsection{Electric Polarization in Ar with Light}

Figure 6.1a shows the optical image of a granular film of MHHP with interdigitated gold contacts on the top. The film was spin-coated on a glass substrate by hot-casting method. Figure $6.1 \mathrm{~b}$ depicts the experimental setup for the built-in potential measurement under the various conditions. The setup consists of an external potentiostat connected in the system and spectrally filtered light from a monochromator $(740 \mathrm{~nm}$ with power $2.5 \mathrm{~mW}$

$\mathrm{cm}^{-2}$ ) constantly incident on the sample. The AC photocurrent was measured as a function of applied potential to find out the built-in potential, which was further used to calculate the polarization as described in Chapter 2. Figure 6.1c shows the polarization of the MHHP film under different conditions. The polarization of the MHHP film in the dark remained 

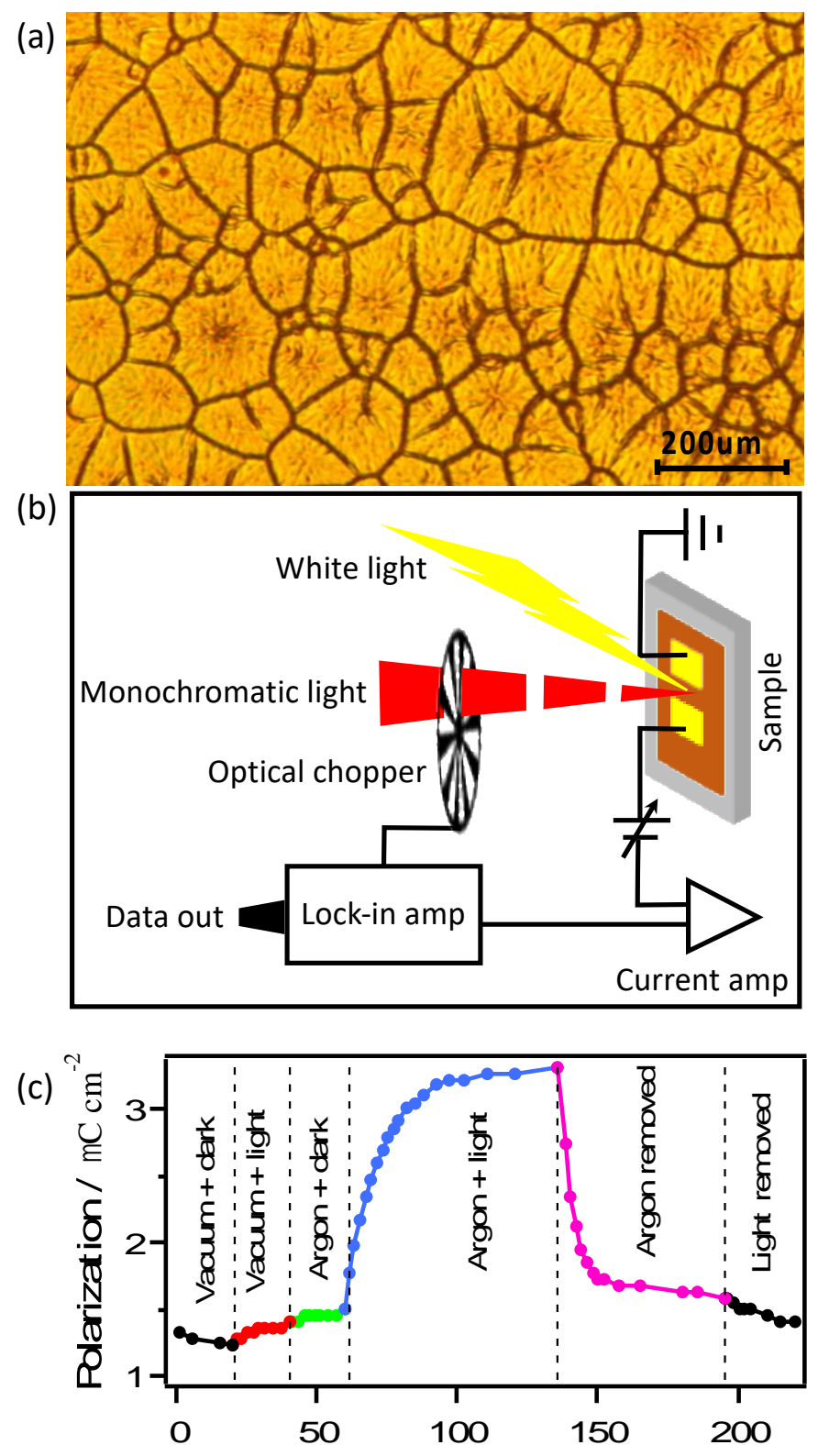

Figure 6.1. (a) Optical image of a granular film of MHHP with interdigitated gold contacts, (b) experimental setup for AC photocurrent measurements, (c) polarization of MHHP thin film. 
almost same both in Ar gas and vacuum while it increased slowly in vacuum with solar light. As soon as the film was exposed to Ar gas with solar light, the polarization kept increasing significantly with increase in the time of exposure with Ar gas. It can be attributed to the polarization of photogenerated carriers, deactivation of defect states, and increasing the polar units in the film. The enhanced polarization should definitely facilitate the photogenerated carrier separation and enhance the photocurrent. Interestingly, when the light was removed, the polarization gradually decreased to the initial value when the vacuum was reestablished.

\subsubsection{AC Photocurrent in Ar with Light}

It is clear that when a film of mixed halide hybrid perovskite is exposed to solar light in Ar gas, the electric polarization is enhanced significantly. We did another experiment to explore the effect of the electric polarization on photocurrent. For this experiment, the monochromatic light at $740 \mathrm{~nm}$ was constantly incident on the device, and the AC photocurrent was measured at zero external potential by changing the background environment as we did while measuring the built-in potential. Figure 6.2 shows the time

dependence of the AC photocurrent. It is very interesting to note here that the trend of variation of the $\mathrm{AC}$ photocurrent is same as the variation of the electric polarization. This clearly proves that the enhancement in the AC photocurrent is due to the enhancement in the electric polarization induced by Ar gas in the presence of solar light. 


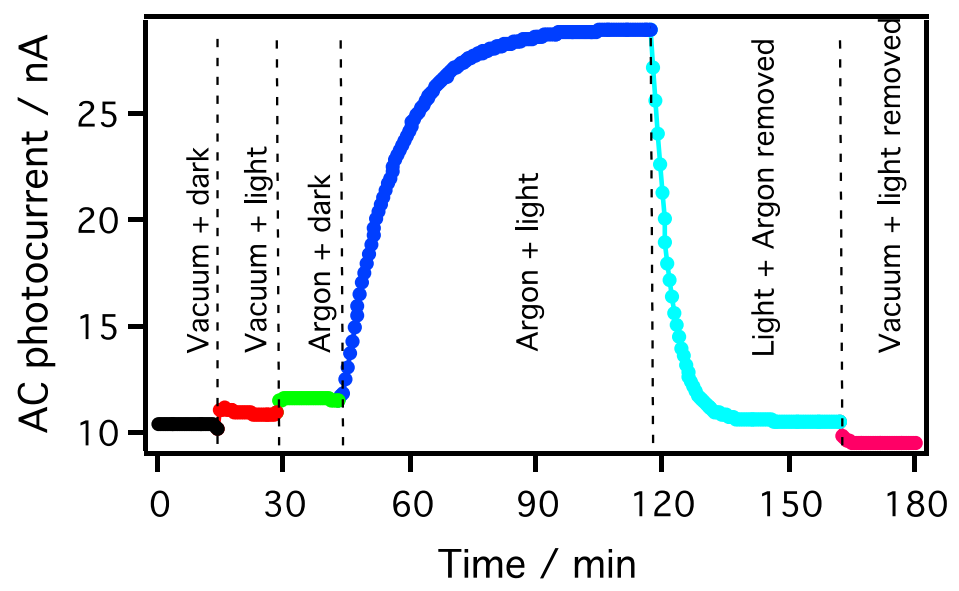

Figure 6.2. Time-dependence of the AC photocurrent in different background environments.

\subsubsection{Effect of Background Gases on the Photocurrent Spectrum}

We carried out different experiments with thin films of MHHP to reveal the influence of different background gases on the photocurrent spectrum. We measured the AC photocurrent spectrum of thin films of MHHP under different background gases using fingerlike interdigitated contacts of gold on top of the perovskite film as before without any external potential. The setup is shown again in Figure 6.3a. As shown in Figure 6.3b, there is a slight enhancement in the photocurrent while going from vacuum to Ar gas in the dark. Surprisingly, when the film was exposed to extra light from a solar simulator, the photocurrent became enhanced by more than one order of magnitude. The enhancement comes from the combined effect of Ar gas and light. The enhanced spatial separation of electron-hole pairs occurs with the introduction of extra energy from the solar light. This spatial separation of these charge carriers is very important to avoid recombination, which in turn directly affects the photocurrent and photovoltage of PSCs. As time increases, the photocurrent reaches a stable maximum, which decreases back close to the original photocurrent in Ar when the light is removed. 
a.

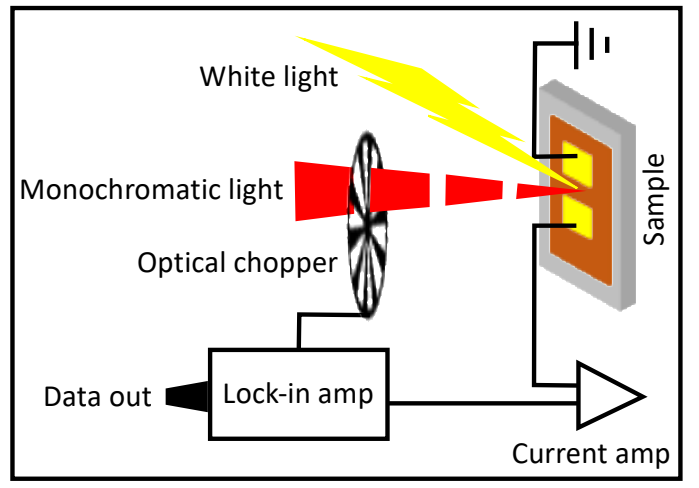

b.

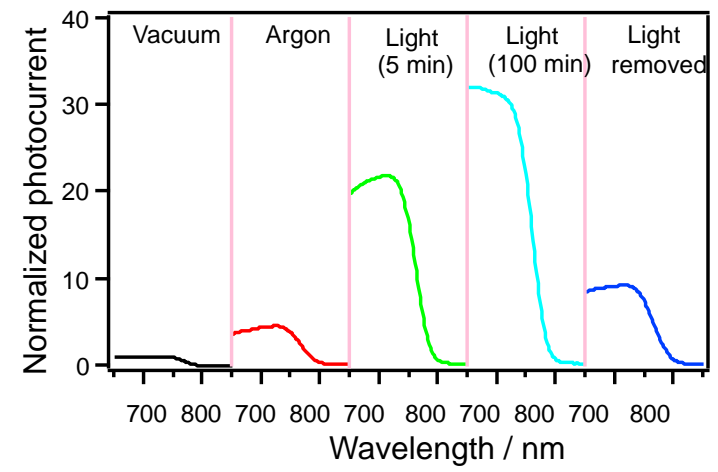

Figure 6.3. (a) Experimental setup for AC photocurrent spectrum measurements, and (c) $\mathrm{AC}$ photocurrent response in vacuum and Ar gas.

Notably, the opto-electronic characteristics of MHHP are preserved when exposed to extra light in an environment of Ar gas. There is no chemical bonding of perovskite with Ar gas, so the films are stable in an Ar environment. We observed the preservation of the perovskite film in other inert gases too (Figure 6.4), however, the enhancement in photocurrent was best for Ar gas. It is interesting to note that He gas did not show any effect on the photocurrent. 


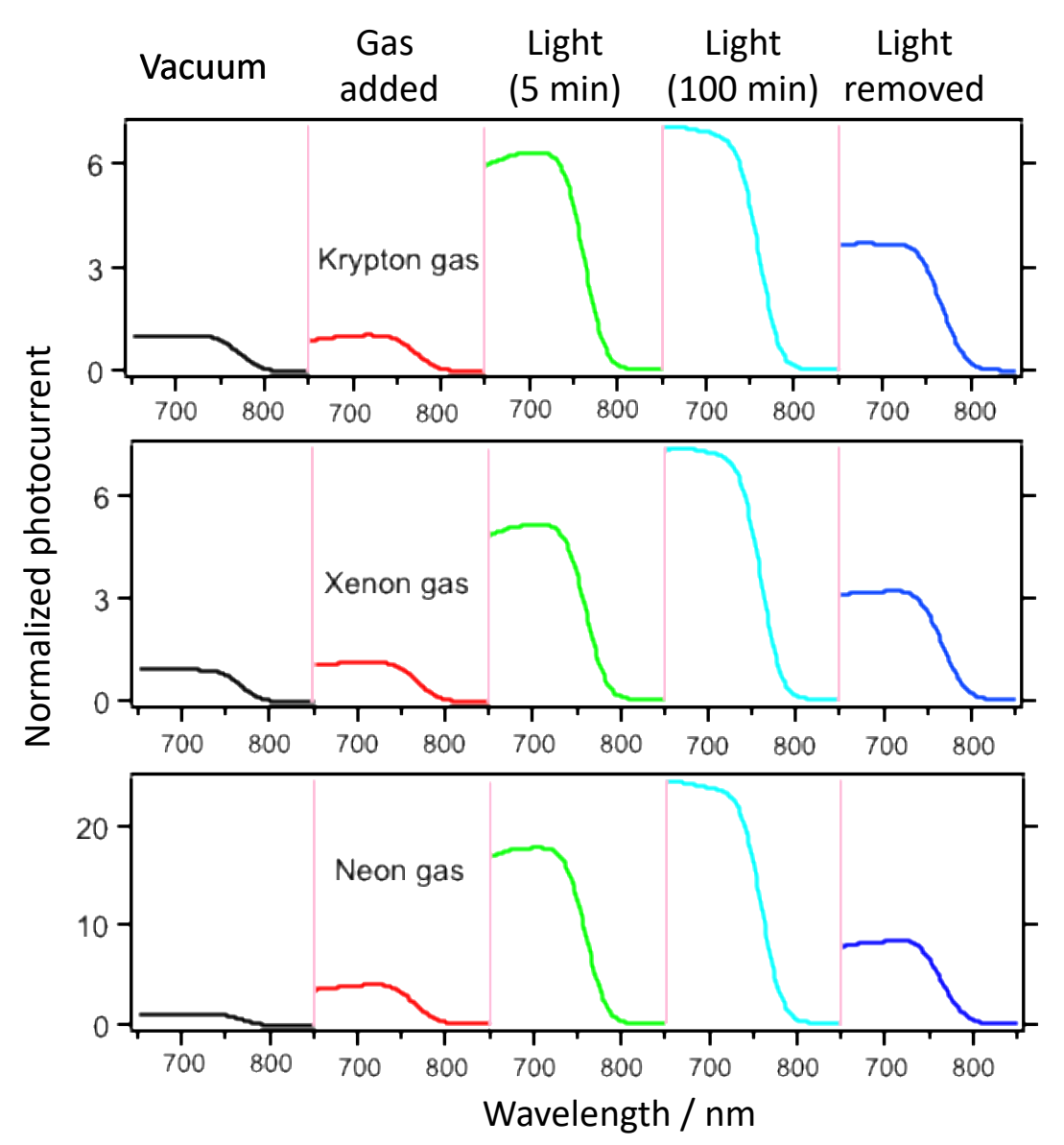

Figure 6.4. AC photocurrent spectrum in different inert gases.

Surface trap states provide recombination pathways and greatly reduce the detected photocurrent. ${ }^{219}$ It has been reported that these surface traps can be cured by exposing the perovskite film to air or oxygen. ${ }^{220}$ The improvement is thought to be due to the reaction of recombination centers with $\mathrm{O}_{2}$ or $\mathrm{OH}^{-}$groups. The removal of recombination pathways by exposure to oxygen/air is temporarily beneficial to solar cell efficiency, since less recombination leads to a higher percentage of charge being collected by the contacts. However, over time, perovskite solar cells degrade as the air reacts with the perovskite. Figure 6.5 reveals that thin film of MHHP showed higher current as soon as the film was exposed to air with light. This enhancement seems to be originating from the near IR region. This can be attributed to the generation of photogenerated carriers from the perovskite film and others which are generated by the reaction of perovskite with air/oxygen in the presence of light. Although the peak current is higher relative to vacuum, there is no 
enhancement in the photocurrent at the band edge. It indicates that this is not a beneficial change for the solar cell application. After 5 minutes of exposure, the film started to show lower and lower photocurrent with increasing time of exposure. After about 100 minutes, the photocurrent spectrum showed completely different characteristics, which indicates complete degradation of the material due to the reaction of perovskite with air and oxygen in the presence of light. The degradation of the perovskite film when exposed to air/oxygen gas might be due to the generation of super oxide $\left(\mathrm{O}_{2}^{-}\right)$, which could react with MA components to produce $\mathrm{PbI}_{2}, \mathrm{I}_{2}$ and $\mathrm{CH}_{3} \mathrm{NH}_{2}{ }^{221}$ These results are in agreement with the reports mentioned elsewhere. ${ }^{222}$

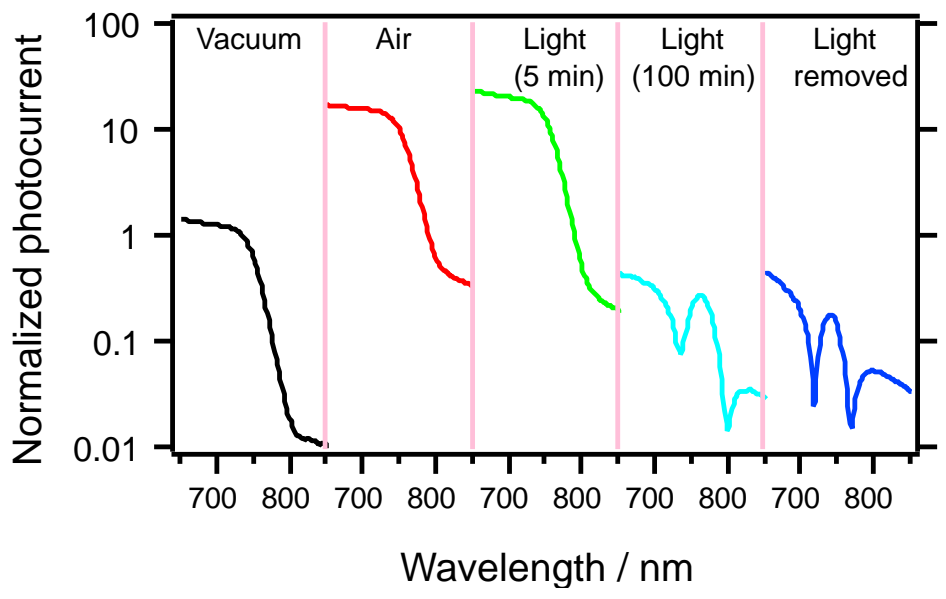

Figure 6.5. AC photocurrent spectrum of MHHP film device in air after different times of exposure.

\subsubsection{Quartz Crystal Microbalance (QCM) Measurements}

To explore whether the perovskite film adsorbs Ar gas during light illumination, we used a quartz crystal microbalance (Gamry eQCM 10M) to measure the mass of the film under Ar gas with and without the presence of light. The quartz crystal with a resonant frequency of $10 \mathrm{MHz}$ was used as a substrate for spin-coating a thin layer $(\sim 400 \mathrm{~nm})$ of hybrid perovskite as described in Chapter 2. The resonant frequency decreases with an 
increase in mass of the crystal due to the adsorption of gases. The amount of gas adsorbed by a thin film in terms of mass can be estimated by using the Sauerbrey equation: ${ }^{223}$

$$
\Delta f=-\frac{2 f_{0}^{2}}{A \sqrt{\rho_{q} \mu_{q}}} \times \Delta m
$$

where $\Delta f$ is the change in the resonant frequency, $f_{0}$ is the fundamental frequency of a bare QCM crystal, $\Delta m$ is the change in mass, $A$ is the area, $\rho_{q}$ and $\mu_{q}$ are the density and shear modulus of the quartz crystal, respectively.

Equation 6.1 can also be written as

$$
\Delta f=-C_{m} \times \Delta m
$$

where $\mathrm{C}_{\mathrm{m}}$ is the calibration constant for the crystal. It is $226 \mathrm{~Hz} \mathrm{~cm}^{2} \mu \mathrm{g}^{-1}$ for our $10 \mathrm{M}$ quartz crystal (Gamry's Instrument). Equation 6.2 shows that the resonant frequency of quartz crystal decreases in proportion to the added mass.

Figure 6.6 shows the RF of QCP in the dark and light with Ar and Vacuum. The RF in vacuum is $29 \mathrm{~Hz}$ higher in the light than in the dark. We believe it is because of increase of temperature. We observed that the RF in dark with Ar is lower than with vacuum. It can be because of Ar pressure. Unlike in vacuum, when the QCP is exposed to Ar with solar light constantly shining on the film, we found the RF decreases by $9 \mathrm{~Hz}$ relative to the RF in the dark. This observation indicates that the mass of QCP increases when the film is exposed to solar light in the presence of Ar. It is because of the adsorption of Ar gas. We did the same experiment with $\mathrm{He}$ and $\mathrm{N}_{2}$ as the background gases and found that the RF increases by about $29 \mathrm{~Hz}$ in $\mathrm{He}$ while going from dark to light (Figure 6.7, blue trace), which is same as the changes observed in vacuum (Figure 6.6). This infers that there is no adsorption of $\mathrm{He}$ gas on the film. This can a reason why there was no enhancement in the AC photocurrent in He gas. There is increase in the RF by about $25 \mathrm{~Hz}$ while going from 
dark to light in $\mathrm{N}_{2}$ gas (Figure 6.7, green trace). There is possibility of adsorption of N2 gas molecules on the film. Values of the RF in various conditions are summarized in Table 6.1. If we consider $29 \mathrm{~Hz}$ increase in the RF while going from the dark to the light, decrease in the RF comes to be 0 for $\mathrm{He}, 4 \mathrm{~Hz}$ for $\mathrm{N}_{2}$ and $38 \mathrm{~Hz}$ for Ar. Using equation 6.2, the amount of gas adsorbed on the film comes out to be $17.9 \mathrm{ng} \mathrm{cm}^{-2}$ for $\mathrm{N}_{2}$ and $168.1 \mathrm{ng} \mathrm{cm}^{-}$ 2 for Ar.

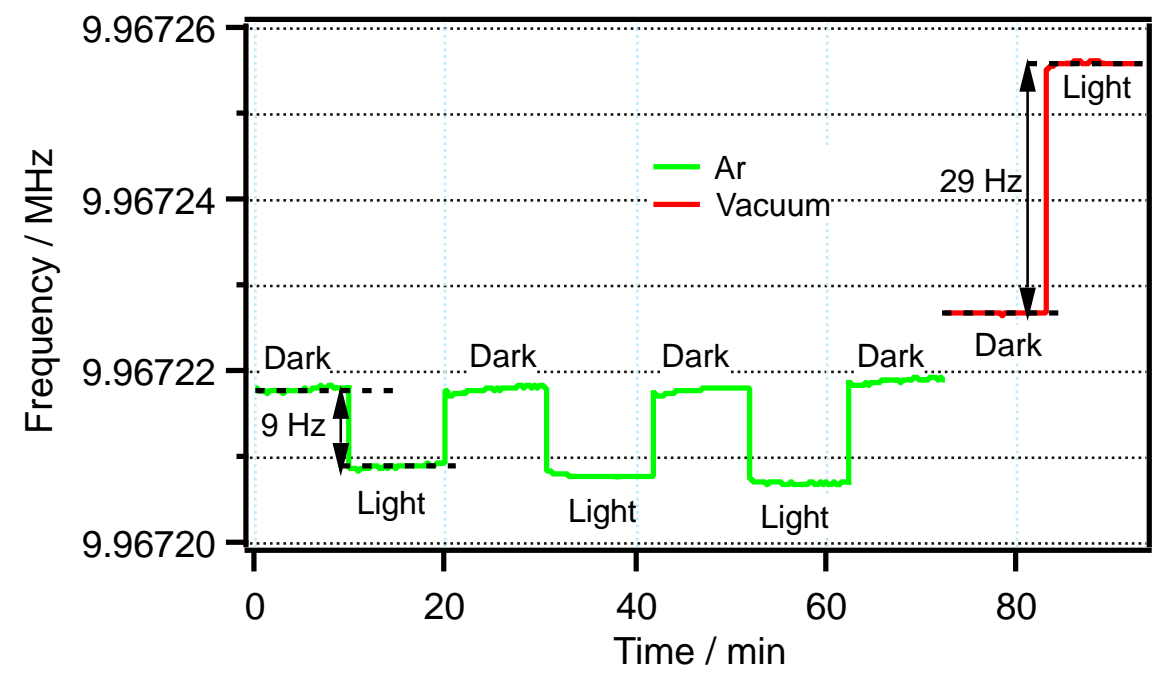

Figure 6.6. Changes in the resonant frequency of QCP in dark and light when the background is vacuum (red trace) and Ar (green trace).

Table 6.1. Resonance frequencies $(\mathrm{Hz})$ of QCP in dark and light with different backgrounds

\begin{tabular}{|l|l|l|l|l|}
\hline Conditions & Vacuum & Argon & Nitrogen & Helium \\
\hline Dark & 9.967227 & 9.967218 & 9.967219 & 9.967221 \\
\hline Light & 9.967256 & 9.967209 & 9.967244 & 9.967250 \\
\hline
\end{tabular}




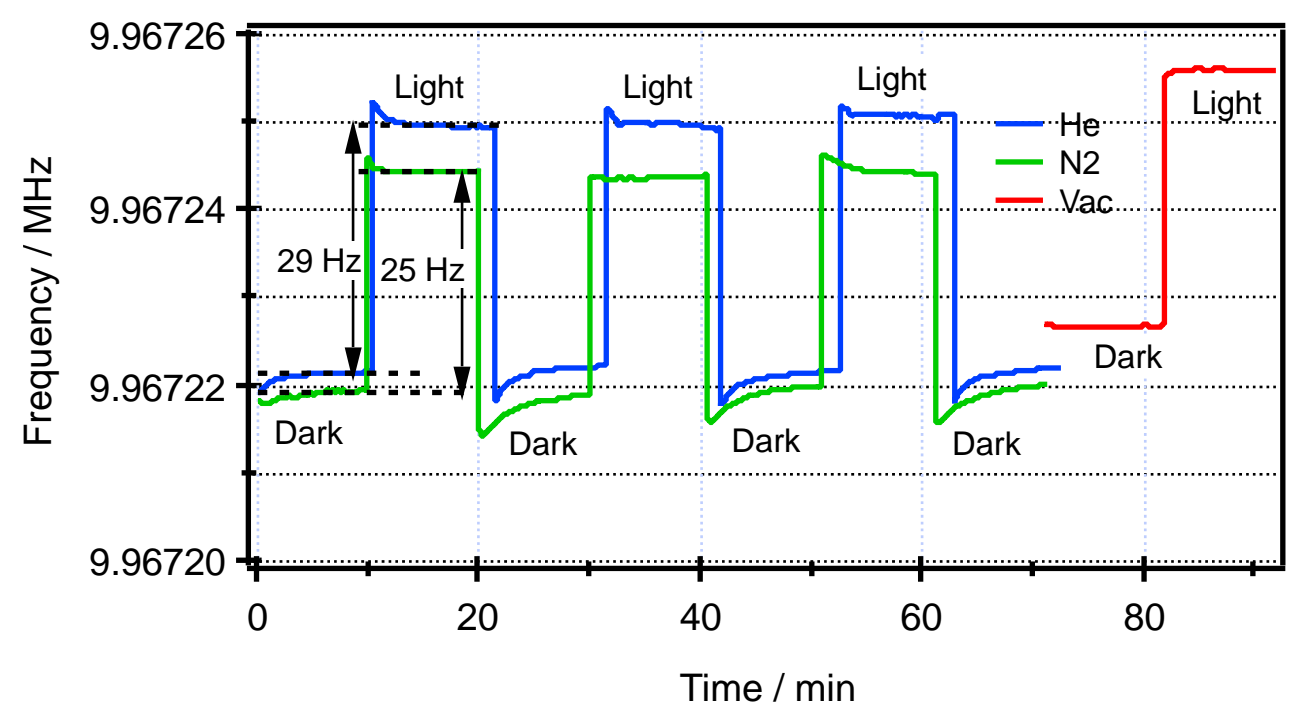

Figure 6.7. Changes in the RF of QCP in dark and light when the background gas is He (blue trace) and $\mathrm{N}_{2}$ (green trace). The RF in vacuum (red trace) is shown for comparison.

\subsubsection{Effect on the Photovoltaic Performance}

Now, it is clear that MHHP film adsorbs Ar gas in the presence of solar light and enhances the polarization of the film thereby increasing the AC photocurrent. We then tested this observation on a real PSC made up of MHHP as a photo-absorber. Figure 6.8a is the optical image of a highly granular film of MHHP spin-coated by the hot casting technique. The architecture of the PSC is shown in Figure 6.8b. It has a planar p-i-n configuration ITO/PEDOT.PSS/perovskite/C60/Au. 
(a)

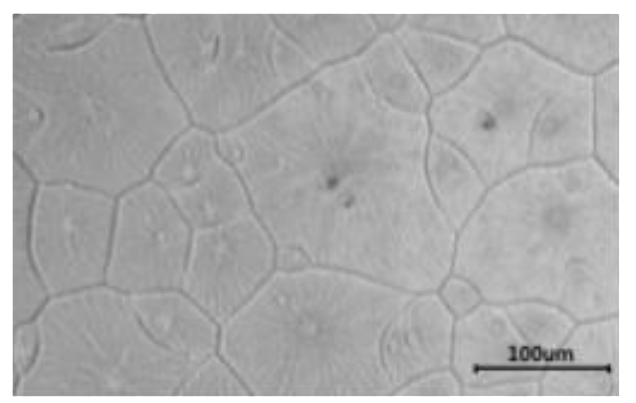

(b)

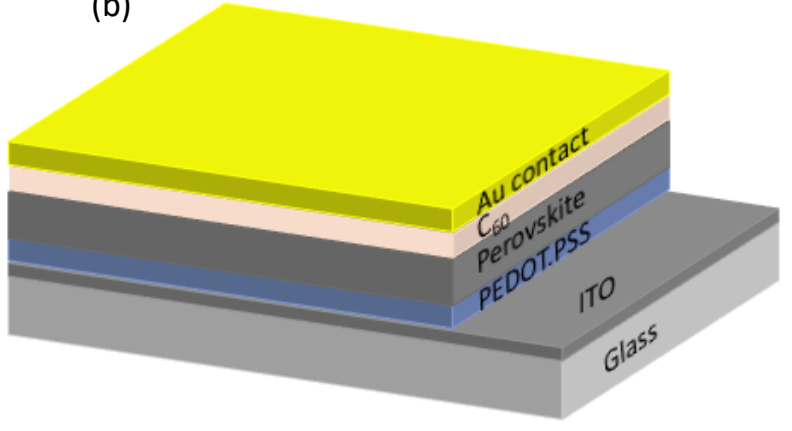

Figure 6.8. (a) Highly granular film of MHHP film spin-coated by hot-casting, and (b) architecture of perovskite solar cell.

The bare ITO is used as the bottom contact and gold film deposited on top of the PEDOT.PSS/MHHP/C 60 is the top contact. The J-V curves of the MHHPSC in the presence of light immediately after exposure to light and after 30 minutes of exposure are shown in Figure 6.9a. There is a small enhancement in the photocurrent, which might be due to light soaking as mentioned elsewhere. ${ }^{211-214}$ The J-V curves in the dark with and without Ar are shown in Figure $6.9 \mathrm{~b}$. The device did not show any photovoltaic improvement in the dark with Ar, other than a slightly higher open circuit potential $\left(\mathrm{V}_{\mathrm{oc}}\right)$. After applying a continuous flow of Ar to the cryostat chamber in the presence of solar light, both the photocurrent and photovoltage increased with an increase in Ar exposure time as shown in Figure 6.10a. The photocurrent increased from 12.9 to $13.6 \mathrm{~mA} \mathrm{~cm}^{-2}$ after 30 minutes of exposure to light in vacuum (Figure 6.9a) while the enhancement is from 13.6 to $16.1 \mathrm{~mA}$ $\mathrm{cm}^{-2}$ for exposure to Ar with light for the same time (Figure 6.10a). Interestingly, the photocurrent became saturated after one hour in light and $\mathrm{Ar}$ gas with a maximum photocurrent of $20.1 \mathrm{~mA} \mathrm{~cm}^{-2}$ and open circuit potential of $860 \mathrm{mV}$. It is clear that light soaking alone cannot explain this enhancement. The role of background gas must be considered to fully understand the reason. Upon removal of Ar gas and reestablishment of vacuum in the cryostat chamber, the photocurrent reversed back close to the original value in vacuum (Figure 6.10b). The variation in photocurrent and efficiencies in the various conditions of measurements are shown in Figure 6.11. 
(a)

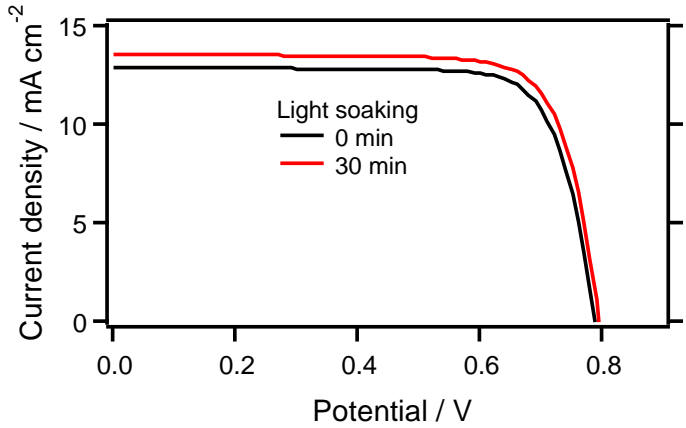

(b)

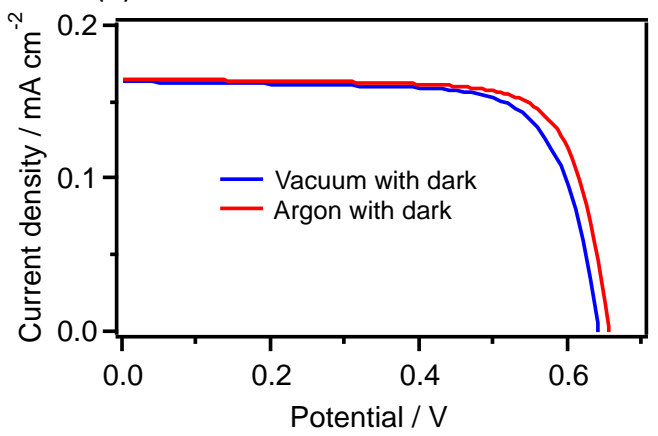

Figure 6.9. J-V curves of perovskite solar cell when the cell is exposed to (a) light in vacuum for different times, and (b) Ar gas and vacuum in the dark.

(a)

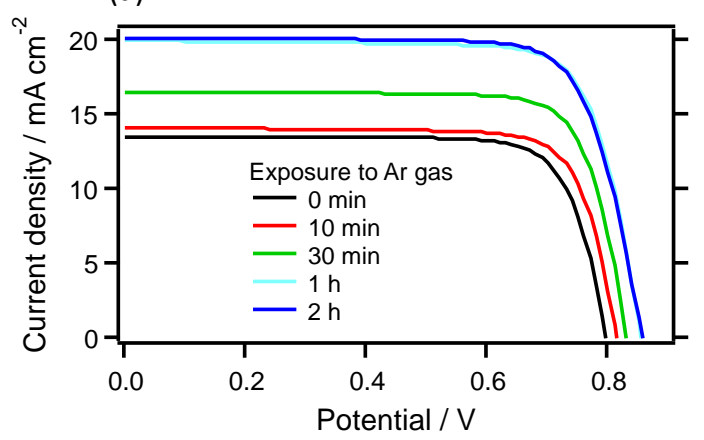

(b)

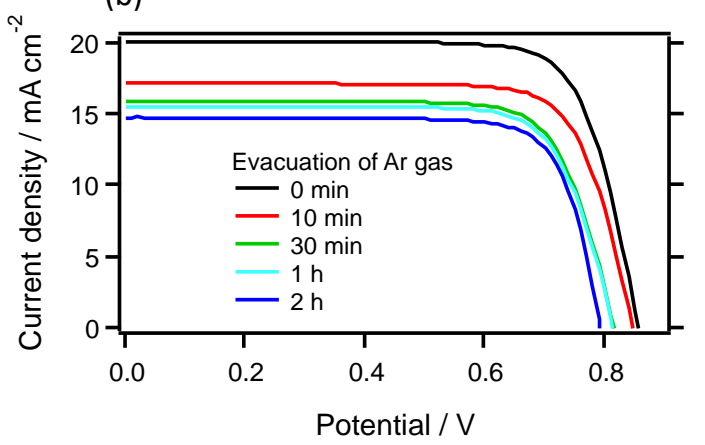

Figure 6.10. J-V curves of perovskite solar cell when the cell is exposed to (a) light under Ar gas environment, and (b) light with evacuating Ar gas for different times. 


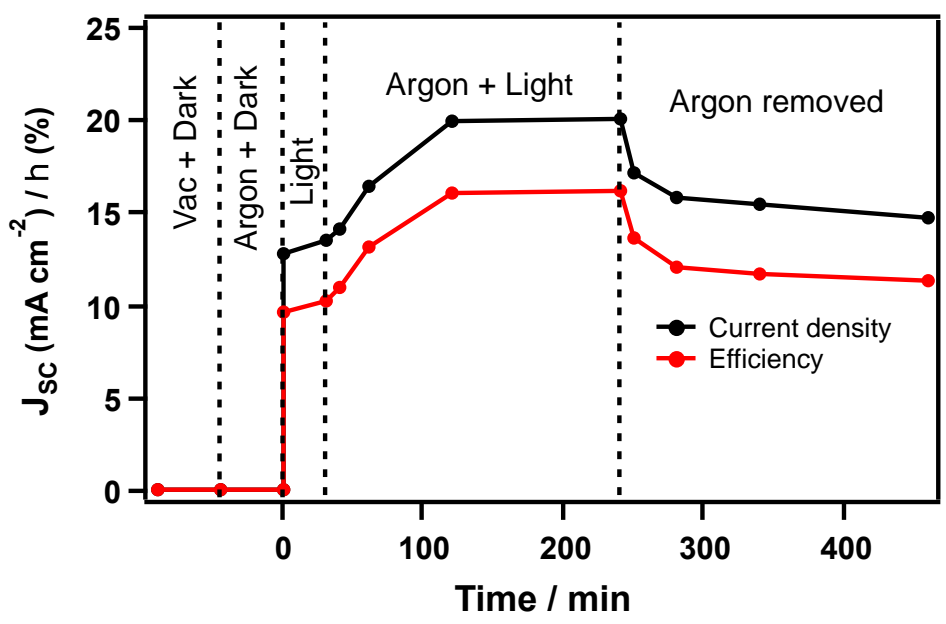

Figure 6.11. Variation of short-circuit current density and efficiency under the different conditions of measurements.

The increased photocurrent density and increased open circuit potential in the $\mathrm{J}-\mathrm{V}$ plot upon exposure to light under Ar are exciting discoveries. These can be attributed to various factors, such as passivation of defect sites by photogenerated carriers, ${ }^{214,} 224$ reduction in chemical resistance and capacitance at the MHHP/contact interfaces, ${ }^{213}$ and lattice expansion induced by light soaking. ${ }^{224}$ Lattice expansion may allow for Ar gas adsorption, where the adsorbed Ar atoms penetrate deep inside the film and passivate the defect sites which were not cured by light soaking. This is a speculation at this point, but it is clear that the effect of Ar are real.

\subsection{Discussions}

The various factors which contribute to the origin of polarization in MHHP thin films are well explained in Chapter 5. The polarization of a MHHP film strongly depends on the size of grains. It increases with increase of the grain size in the film. Our results reveal that this polarization is significantly enhanced when the film is exposed to Ar gas with solar light. Solar light causes the lattice expansion as mentioned elsewhere. ${ }^{224}$ The enhanced polarization then facilitates the separation of excitons to increase the photocurrent. An 
inert gas should not readily react with the perovskite surface; however, the surface field can generate a local dipole on inert gas molecules. ${ }^{225}$ This will attract the inert gas molecules and lead to physisorption of the gas molecules on the perovskite. Once adsorbed, the inert molecules remain attached with an activation energy that increases with the molecular weight and polarizability of the exposed molecule, as has been determined for noble gas adsorption on a variety of semiconductor surfaces. ${ }^{226}$ The combination of inert gas plus solar illumination appears to be effective at neutralizing surface recombination centers without damaging the perovskite film, with Ar being the best. No light induced increase in the photocurrent is observed when helium is used as the inert gas. This is most likely because the binding energy of the helium for physisorption on the perovskite is below the thermal energy.

In fact, it is known from the literature that the solar cell efficiency can be improved temporally by exposure to high intensity light, ${ }^{227}$ which becomes incomplete without any explanation for the role of background environment. Explanations for this effect are that the light soaking neutralizes the surface traps ${ }^{228}$ or increases the open circuit voltage due to charge accumulation at the interfaces. ${ }^{211}$ High quality perovskite solar cells are typically fabricated in an Ar environment ${ }^{229}$ and then encapsulated, so it is possible that the effect we observe here is due to the same mechanism.

\subsection{Conclusions}

In conclusion, it is shown that the MHHP film adsorbs Ar gas in the presence of solar light. Ar gas with the solar light results in the enhancement of the electric polarization of the MHHP film. Consequently, the photocurrent is increased due to the stronger driving force for the separation of excitons. This observation is illustrated in a real PSC where the photovoltaic enhancement is observed with Ar gas. Our results suggest that the contribution from the background environment should be taken into consideration when describing the photovoltaic performance of a PSC. The parameters of our cell are not great, but they are enough to explain the observations. How Ar atoms interact with the perovskite film in the presence of solar light, and theoretical explanation is a very interesting topic for future studies. 


\section{CHAPTER VII}

\section{SYNTHESIS AND CHARACTERIZATION OF LAYERED HYBRID PEROVSKITES}

\subsection{Introduction}

Three-dimensional (3D) films of hybrid perovskites have been used for photovoltaic applications with a photoconversion efficiency surpassing $25 \%,{ }^{51}$ however, they suffer from insufficient long-term stability and toxicity of the lead most commonly used as a component of the perovskite crystal. ${ }^{90,230}$ The problem related to the toxicity of $\mathrm{Pb}$ can be resolved by using a more environmentally friendly metal, such as $\mathrm{Sn} .{ }^{231-233}$ The issue with instability under ambient conditions, including heat and light, is still not resolved. Smith et $a l .{ }^{100}$ and Cao et al. ${ }^{101}$ showed that two-dimensional (2D) iodide-based layered perovskites are more stable than the corresponding 3D films. The 2D layered perovskites are formed when 3D frameworks are sliced into well-defined 2D single layer sections. The 2D perovskites have the general formula of $\left(\mathrm{RNH}_{3}\right)_{2}(\mathrm{~A})_{\mathrm{n}-1} \mathrm{M}_{n} \mathrm{X}_{3 \mathrm{n}+1}$ where $\mathrm{RNH}_{3}$ is a large aliphatic or aromatic alkylammonium cation acting as a spacer between the $2 \mathrm{D}$ perovskite layers, $\mathrm{A}$ and $\mathrm{M}$ are cations and $\mathrm{X}$ anions which form the perovskite framework, and $\mathrm{n}$ represents the number of corner-sharing $\left[\mathrm{MX}_{6}\right]^{4-}$ octahedral layers sandwiched between interdigitating bilayers of long-chain alkylammonium ions as described in Chapter $1 .^{102}$ 
The larger hydrophobic cations used in the synthesis are responsible for the new phases of perovskite being formed, leading to the $2 \mathrm{D}$ nature, but it also leads to significant improvement in the moisture stability of the films. ${ }^{234}$ The improved stability under ambient conditions is an excellent feature of the $2 \mathrm{D}$ perovskites that has led to them receiving increased attention over recent years.

\subsection{Experimental Section}

The synthesis of crystals of 2D and 3D hybrid perovskites is given in Chapter 2.

\subsubsection{Preparation of Precursor Solution}

The precursor solution of each type of layered perovskite for the film fabrication was prepared from the dry crystals prepared as described in Chapter 2. The crystals were dissolved in anhydrous DMF to make a $0.4 \mathrm{M}$ solution with respect to lead content and heated at $70{ }^{\circ} \mathrm{C}$ with stirring for 30 minutes.

\subsubsection{Device Fabrication}

All the devices described in this chapter were made by spin-coating the precursor solution at $70{ }^{\circ} \mathrm{C}$ on a glass substrate pre-heated at $150{ }^{\circ} \mathrm{C}$. Briefly, a pre-cleaned glass slide was heated at $150{ }^{\circ} \mathrm{C}$ for $5 \mathrm{~min}$ and quickly transferred to the top of a spin-coater. $150 \mu \mathrm{L}$ of the precursor solution was dropped to the center of the slide and spin-coated at 3000 rpm for 30 seconds. The device was heated at $100{ }^{\circ} \mathrm{C}$ for $10 \mathrm{~min}$ for the complete conversion of the perovskite and removal of the solvent if any. This device was used for UV/VIS and XRD measurements. For the device used for the AC photocurrent measurements, interdigitated Au contacts were deposited on top of the film as described in Chapter 2. Scheme 7.1 summarizes the device fabrication process. 
Scheme 7.1. Fabrication of devices for AC photocurrent measurements.

Clean the glass substrate sonicating in detergent, acetone, ethanol and isopropanol followed by

UV/ozone treatment.

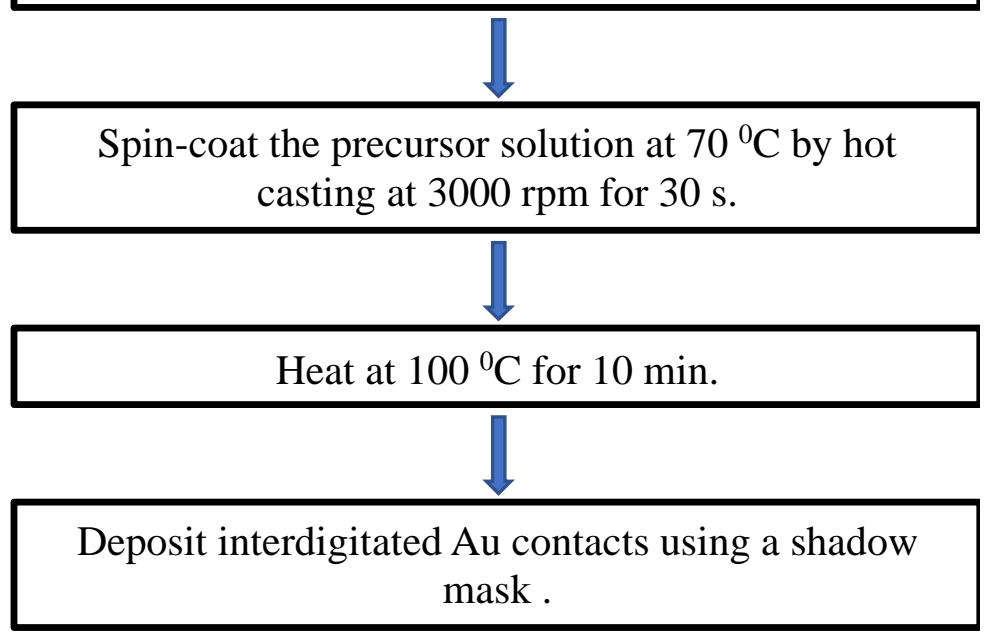

\subsubsection{Characterization with AC Photocurrent Method}

As described in Chapters V and VI, the device with interdigitated Au contacts was loaded into an optical cryostat and the chamber of the cryostat was maintained at a pressure of $10^{-7}$ torr.

\subsection{Results}

As shown in Chapter 2, the crystals of $(B A)_{2} \mathrm{PbI}_{4}(\mathrm{n}=1)$ are orange. These crystals do not have methylammonium ions. (BA $)_{2}(\mathrm{MA}) \mathrm{Pb}_{2} \mathrm{I}_{7}(\mathrm{n}=2)$ crystals are scarlet in color while the crystals for $n=3$ and $n=4$ are black. Figure 7.1a-d shows optical images of the films for $n=1$ through $n=4$. The films are very compact with rectangular-shaped crystals. Apparently, the crystals seem to be larger for $n=1$ in comparison to the films for higher value of $n$. We can see rectangular crystals in the optical images of the films of these materials made by hot casting the precursor solution on the glass substrates. The low angle XRD spectra of these films are depicted in Figure 7.1e. The XRD spectra show the phase purity of the crystals consistent with the literature and the successful synthesis of 2D perovskites with 
controlled $n$ values between 1 and 4 . The number of reflections below $2 q=12^{0}$ is equal to the $\mathrm{n}$ value, consistent with other reports. ${ }^{102,234}$

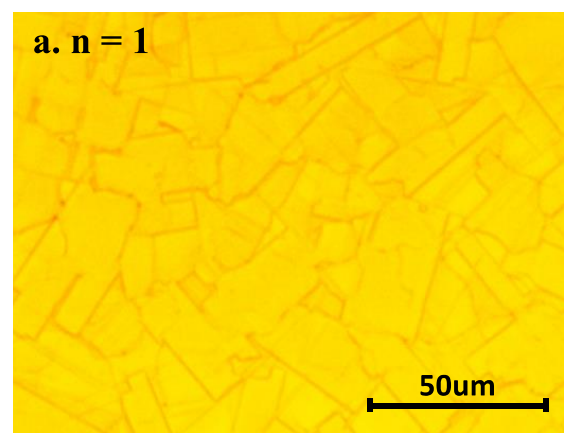

$$
\text { b. } \mathbf{n}=\mathbf{2}
$$
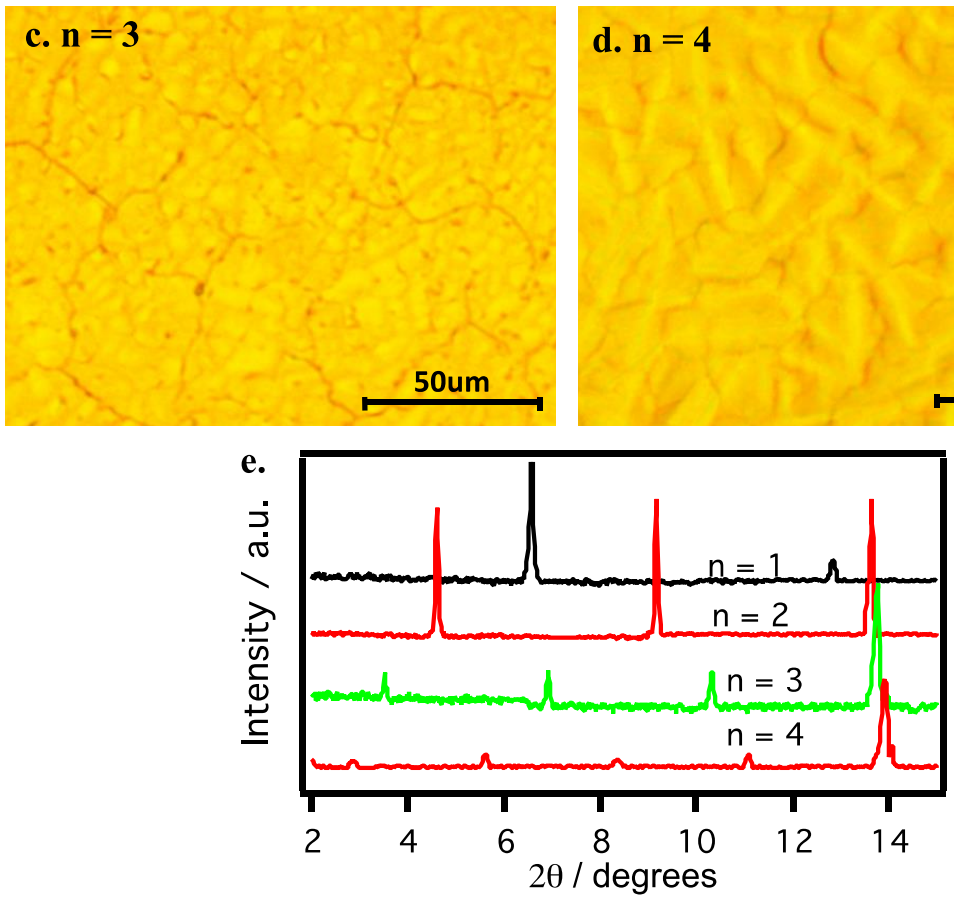

Figure 7.1. (a)-(d) Optical images of the films of different 2D hybrid perovskites, and (e) low angle XRD spectra of these perovskites spin-coated on the glass substrates.

Figure 7.2. shows further evidence of the successful synthesis of the 2D perovskites in the optical absorbance spectra of the films on the glass substrate made by hot-casting method. The optical bandgap decreased from $n=1(2.43 \mathrm{eV})$ to $n=4(1.92 \mathrm{eV})$, consistent with the literature. ${ }^{102}$ 


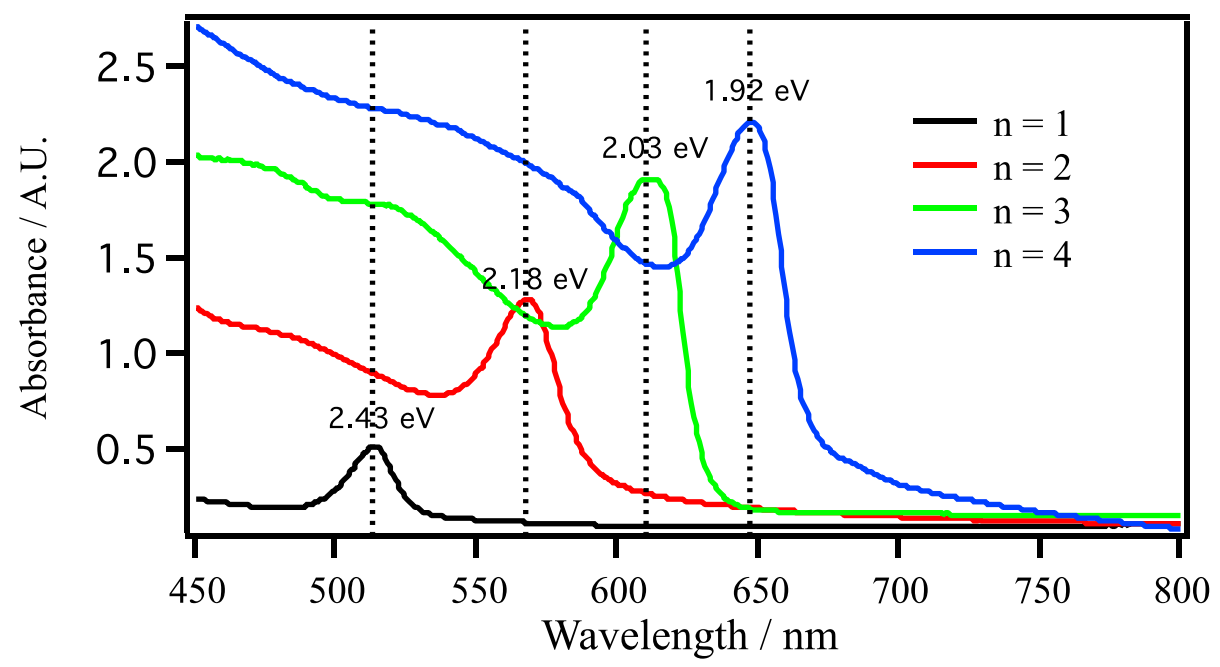

Figure 7.2. Optical absorbance spectra of different types of 2D hybrid perovskites.

The films of different $2 \mathrm{D}$ perovskites were then characterized with the $\mathrm{AC}$ photocurrent technique as was done with 3D MHHP films in Chapters 5 and 6. Figure 7.3.a shows an optical image of the $2 \mathrm{D}$ film for $\mathrm{n}=4$ with interdigitated $\mathrm{Au}$ contacts on the top of the film. We did not get any AC photocurrent for $n=1$ in the absence of external potential. However, when the external potential was increased to $1 \mathrm{~V}$, the $\mathrm{AC}$ photocurrent spectrum (Figure 7.4.b, black trace) was obtained with a clear peak at $2.43 \mathrm{eV}$. This indicates that there is not intrinsic polarization of the film for $n=1$. Figure 7.4.b (red trace) shows the AC photocurrent spectrum for $\mathrm{n}=2$ for zero external potential. This film showed some internal polarization of the film. The peaks for $n=1$ and 2 are characteristic to the bandgap of these perovskites consistent with the optical absorbance data shown in Figure 7.2. Figure 7.3c-d are the AC photocurrent spectra for $n=3$ and 4 respectively. We observe that the optical absorption range keeps increasing with increase in the value of $n$. Further research about the polarization and optoelectronic properties of these emerging perovskites is going on. 
a.

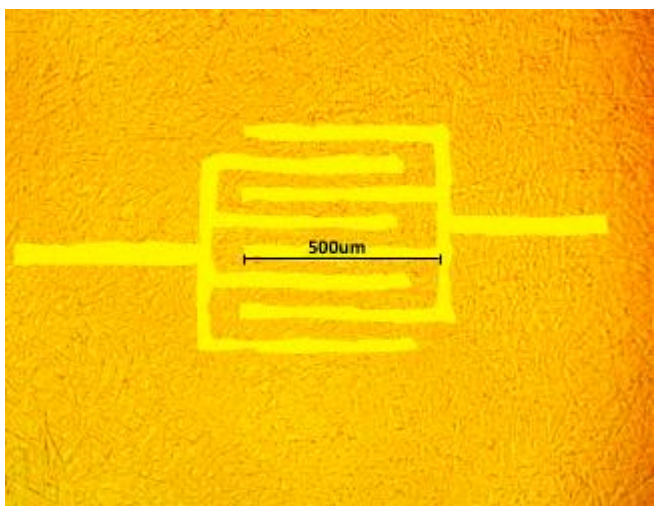

C.

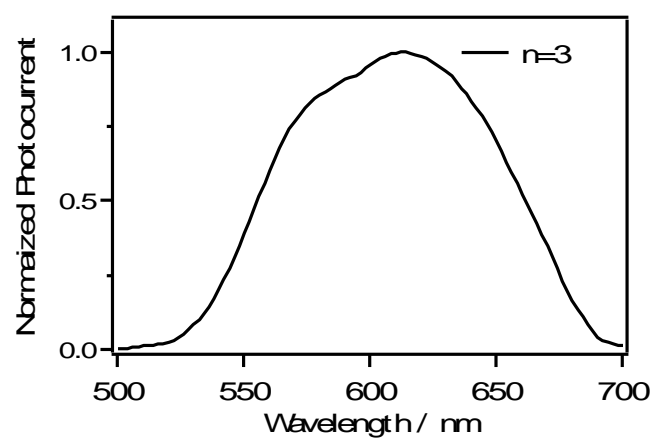

b.

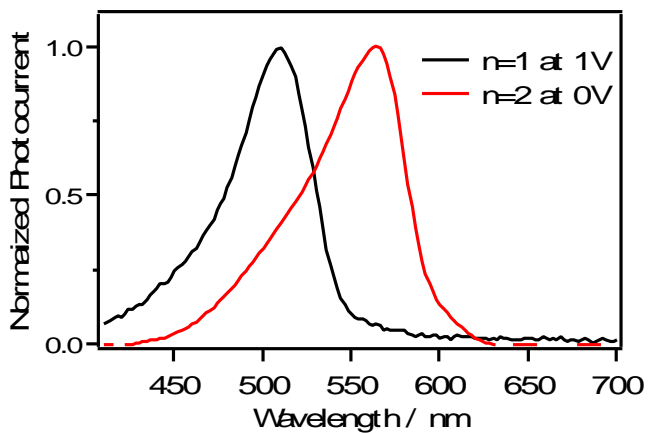

d.

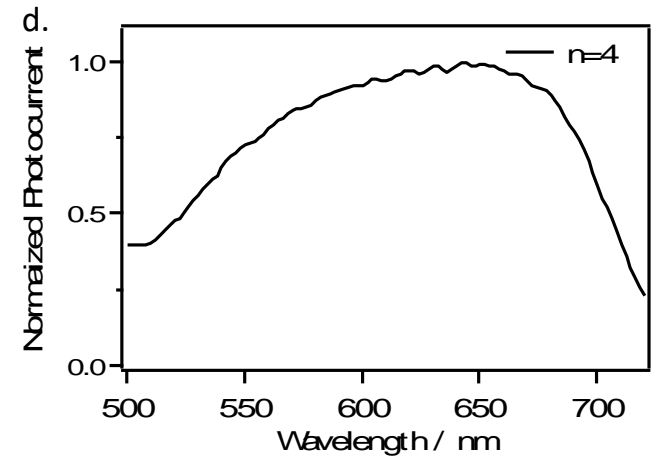

Figure 7.3. (a) Optical image of the film for $n=4$ with interdigitated $A u$ contacts used for the AC photocurrent measurement, (b)-(d) the AC photocurrent spectra of the device for $\mathrm{n}=1$ to 4 . 


\section{CHAPTER VIII}

\section{CONCLUSIONS AND FUTURE DIRECTIONS}

\subsection{Conclusions}

(a) REO nanoparticles enhance the photovoltaic performance of dye-sensitized solar cells.

Commonly used semiconductor titania was doped with REO $\left(\mathrm{Er}_{2} \mathrm{O}_{3}\right.$ and $\left.\mathrm{Nd}_{2} \mathrm{O}_{3}\right)$ nanoparticles and highly reproducible DSSCs were fabricated and photoelectrical characterization was carried out to study their photovoltaic performance. It is shown in chapter 3 that the doping of mesoporous $\mathrm{TiO}_{2}$ with both $\mathrm{REO} \mathrm{Er}_{2} \mathrm{O}_{3}$ and $\mathrm{Nd}_{2} \mathrm{O}_{3}$ nanoparticles showed significant improvement in the photovoltaic performance of DSSCs through the increased dye loading due to the increased surface area, and the enhanced electron transport efficiency. Photoelectrical and electrochemical characterization of REOdoped titania and undoped titania in Chapter 4 reveals that REO nanoparticles reduce the number of deep traps or converts the deep traps to shallower traps from where electrons can easily get de-trapped. Consequently, the reduction in trap state density is also evident in the decrease in flat band potential, and reduction in capacitance of the titania film when doped with REO nanoparticles. The increase in lifetime of the transport carriers provided by the decreased trap density provides an explanation for the reduced electrical impedance of the REO doped film, and also partially explains the improved performance of REOdoped titania in photocatalytic and solar cell applications. 
(b) The electric polarization of mixed halide hybrid perovskite facilitates the excitonic separation, and this electric polarization can be measured by using an AC photocurrent measurement.

Mixed halide hybrid perovskites are stacked as $\left[\mathrm{PbCl}_{\mathrm{x}} \mathrm{I}_{6-\mathrm{x}}\right]$ octahedral units, sharing the corners, which form cuboctahedral sites where MA cations are caged. The mismatch between the ionic radii of the different species forming a unit cell leads to structural distortions in perovskites such as tilting and axial rotation of the corner-sharing octahedra, and these lower the symmetry of the structure. The incorporation of $\mathrm{Cl}$ in iodide-based hybrid perovskites distorts the unit cell from the ideal cubic perovskite structure causing destruction of the centrosymmetric nature. It can lead to polar or anti-polar unit-cells. Antipolar units cancel the effect of one another while polar units add up, resulting in a local intrinsic polarization $\mathrm{P}_{0}$. Grain screen the effect of intrinsic polarization. As a result, the electric polarization of MHHP decreases with decreases in the grain size. In Chapter 5, the $\mathrm{AC}$ photocurrent measurement technique is used to measure $\mathrm{V}_{\mathrm{bi}}$. The $\mathrm{AC}$ photocurrent is zero at $\mathrm{V}_{\mathrm{bi}}$ and the phase changes by 180 degrees. The intrinsic polarization $\left(\mathrm{P}_{0}\right)$ of samples can be used to check the quality of the film, where a larger $\mathrm{P}_{0}$ means a higher quality film with larger grain sizes. Poling the device above the built-in potential further polarizes the film, which revert back to their original state when the poling is removed. When the device is relaxed, the detrapping of photogenerated charge carriers takes place. In devices with larger grains, the $\mathrm{V}_{\mathrm{bi}}$ helps in detrapping, while in devices with smaller grains, the trapped carriers remain in defect states for longer time because of the screening affect.

(c) Ar gas enhances the electric polarization of mixed halide hybrid perovskite film and leads to the photovoltaic improvement in the perovskite solar cells.

In chapter 6, it is found that the MHHP film adsorbs Ar gas in the presence of solar light. The solar energy causes lattice expansion, and Ar gas with the solar light results in the enhancement of the electric polarization of the MHHP film. Consequently, the AC photocurrent is increased due to the reduced recombination by the enhanced intrinsic polarization. This observation is illustrated in a real PSC where the photovoltaic enhancement is observed with Ar gas. Our results suggest that the contribution from the 
background environment should be taken into consideration when describing the photovoltaic performance of a PSC.

\subsection{Directions for Future Studies}

(a) The electrical conductivity of nanostructured titania is enhanced by doping it with REO NPs. Interestingly, there is huge reduction of the trap state density of titania due to the injection of electrons from f-states of REOs to the deep traps of titania. There is no theoretical studies on the effect of REO NPs on titania. Use of titania doped with REO NPs in catalytic systems and as an electron-transport material in the photovoltaic cells can be very promising.

(b) Perovskite based devices are often encapsulated in inert gases, preferably argon and nitrogen for avoiding the degradation of the material due to the contact with air and moisture. It is very interesting that Ar gas can interact with hybrid perovskite and improves the photovoltaic performance. Prolonged exposure of perovskite film with solar light causes lattice expansion resulting in the structural stability and improved photovoltaic performance, but there is no explanation about the involvement of background gases. The study to explore the underlying mechanism for the effect of $\mathrm{Ar}$ and other inert gases on perovskite-based devices is very interesting and can give a direction for various applications. Further studies through theoretical modelling with computational works, photoluminescence studies for physical and chemical transformation of hybrid perovskite crystals, c-AFM studies for surface conductivities, XRD in different inert gases for phase changes and the optoelectronic properties within a grain and across a grain boundary using probe stations can help in achieving the goal.

(c) The experiments of hybrid perovskite with Quart Crystal Microbalance reveal that hybrid perovskite film adsorbs Ar gas in the presence of solar light only. Where is Ar adsorbed? There can be particular sites on the surface or interior of the film where Ar atoms are adsorbed. Energy-Dispersive X-ray Spectroscopy (EDAX) study of perovskite film in Ar with solar light can give an evidence for the enhanced electric polarization, and improved photovoltaic performance. 
(d) It is possible that the interaction of perovskite film with inert gases depends on the intensity of solar light. Larger inert gases can show the similar effect as Ar gas when the intensity of light is high enough to cause lattice expansion for the adsorption for these gases. Light intensity dependence of the electric polarization can be significant for further exploration of interaction of perovskite with inert gases.

(e) 2D hybrid perovskites are promising new photoactive materials. Compared to 3D analogue they are more stable in the ambient conditions. Chemical engineering with more hydrophobic and less insulating spacers and suitable organic cation(s) can help in the development of better 2D materials for optoelectronic applications.

(f) 2D perovskite crystals are weakly bound by the Van del Waals forces and can be cleaved to get individual layers by mechanical or chemical exfoliations. The study of electron transport properties of molecular layers of 2D materials can give further directions for their applications.

(g) We observed that AC photocurrent of films having some intrinsic electric polarization is dependent on the external potential. When the external potential counterbalances the built-in potential, the AC photocurrent is negligible. At this moment, the interaction of phonon-perovskite becomes more significant. Exploration of the interaction of phononperovskite can give a direction for the thermal stability of the perovskite-based devices.

(h) The study of films of other perovskites such as multi-cations perovskites for electric polarization is very important for their applications in the optoelectronic devices. 


\section{REFERENCES}

1. United Nations, Department of Economic and Social Affairs, Population Division (2019). World Population Prospects 2019: Data Booklet (ST/ESA/SER.A/424).

2. Gerland, P.; Raftery, A. E.; Ševčíková, H.; Li, N.; Gu, D.; Spoorenberg, T.; Alkema, L.; Fosdick, B. K.; Chunn, J.; Lalic, N., World Population Stabilization Unlikely This Century. Science 2014, 346, 234-237.

3. Lewis, N. S.; Crabtree, G.; Nozik, A. J.; Wasielewski, M. R.; Alivisatos, P.; Kung, H.; Tsao, J.; Chandler, E.; Walukiewicz, W.; Spitler, M. Basic Research Needs for Solar Energy Utilization. Report of the Basic Energy Sciences Workshop on Solar Energy Utilization, April 18-21, 2005; DOESC (USDOE Office of Science (SC)): 2005 .

4. Islam, M. T.; Shahir, S.; Uddin, T. I.; Saifullah, A., Current Energy Scenario and Future Prospect of Renewable Energy in Bangladesh. Renew. Sust. Energy Rev. 2014, 39, 1074-1088.

5. Shafiee, S.; Topal, E., When will Fossil Fuel Reserves be Diminished? Energy Policy 2009, 37, 181-189.

6. Renewable Energy Policy Network for the 21st Century. Renewables 2018 Global Status Report; 2018.

7. Schiermeier, Q.; Tollefson, J.; Scully, T.; Witze, A.; Morton, O., Energy Alternatives: Electricity without Carbon. Nature 2008, 454, 816-823.

8. Hagfeldt, A.; Boschloo, G.; Sun, L.; Kloo, L.; Pettersson, H., Dye-Sensitized Solar Cells. Chem. Rev. 2010, 110, 6595-6663. 
9. Bernede, J., Organic Photovoltaic Cells: History, Principle and Techniques. J. Chi. Chem. Soc. 2008, 53, 1549-1564.

10. Green, M. A.; Emery, K.; Hishikawa, Y.; Warta, W.; Dunlop, E. D., Solar Cell Efficiency Tables (Version 45). Prog. Photovol. 2015, 23, 1-9.

11. Rockett, A. A., The Future of Energy-Photovoltaics. Curr. Opin. Solid State Mater. Sci. 2010, 14, 117-122.

12. Wang, W.; Winkler, M. T.; Gunawan, O.; Gokmen, T.; Todorov, T. K.; Zhu, Y.; Mitzi, D. B., Device Characteristics of CZTSSe Thin-Film Solar Cells with $12.6 \%$ Efficiency. Adv. Energy Mater. 2014, 4, 1301465.

13. Günes, S.; Neugebauer, H.; Sariciftci, N. S., Conjugated Polymer-Based Organic Solar Cells. Chem. Rev. 2007, 107, 1324-1338.

14. Anwar, H.; Arif, I.; Javeed, U.; Mushtaq, H.; Ali, K.; Sharma, S., Quantum Dot Solar Cells. In Solar Cells, Springer: 2020; pp 235-258.

15. Gong, J.; Sumathy, K.; Qiao, Q.; Zhou, Z., Review on dye-sensitized solar cells (DSSCs): Advanced techniques and research trends. Renew. Sustainable Energy Rev. 2017, 68, 234-246.

16. Nie, W.; Tsai, H.; Asadpour, R.; Blancon, J.-C.; Neukirch, A. J.; Gupta, G.; Crochet, J. J.; Chhowalla, M.; Tretiak, S.; Alam, M. A., High-Efficiency Solution-Processed Perovskite Solar Cells with Millimeter-Scale Grains. Science 2015, 347, 522-525.

17. O’Regan, B.; Grätzel, M., A low-cost, high-efficiency solar cell based on dyesensitized colloidal $\mathrm{TiO}_{2}$ films. Nature 1991, 353, 737-740.

18. Gonçalves, L. M.; de Zea Bermudez, V.; Ribeiro, H. A.; Mendes, A. M., DyeSensitized Solar Cells: A Safe Bet for the Future. Energy Environ. Sci. 2008, 1, 655667.

19. Nazeeruddin, M. K.; Baranoff, E.; Grätzel, M., Dye-Sensitized Solar Cells: a Brief Overview. Solar energy 2011, 85, 1172-1178.

20. Grätzel, M., Dye-Sensitized Solar Cells. J. Photochem. Photobiol., C 2003, 4, 145153. 
21. Kuang, D.; Ito, S.; Wenger, B.; Klein, C.; Moser, J.-E.; Humphry-Baker, R.; Zakeeruddin, S. M.; Grätzel, M., High Molar Extinction Coefficient Heteroleptic Ruthenium Complexes for Thin Film Dye-Sensitized Solar Cells. J. Am. Chem. Soc. 2006, $128,4146-4154$.

22. Nowotny, M. K.; Sheppard, L. R.; Bak, T.; Nowotny, J., Defect Chemistry of Titanium Dioxide. Application of Defect Engineering in Processing of $\mathrm{TiO}_{2}$-Based Photocatalysts. J. Phys. Chem. C 2008, 112, 5275-5300.

23. Park, N.-G.; Van de Lagemaat, J.; Frank; AJ, Comparison of Dye-Sensitized Rutileand Anatase-Based $\mathrm{TiO}_{2}$ Solar Cells. J. Phys. Chem. B 2000, 104, 8989-8994.

24. Abeysingha, S. N.; Wijesundera, R. P.; Hettiarachchi, C. V.; Perera, I. R., Zn Based 3D-Coordination Polymer as the Photoanode Material in Dye-Sensitized Solar Cells. Mater. Chem. Phys. 2020, 123109.

25. Law, M.; Greene, L. E.; Johnson, J. C.; Saykally, R.; Yang, P., Nanowire DyeSensitized Solar Cells. Nature Mater. 2005, 4, 455-459.

26. Zhang, Q.; Chou, T. P.; Russo, B.; Jenekhe, S. A.; Cao, G., Aggregation of ZnO Nanocrystallites for High Conversion Efficiency in Dye-Sensitized Solar Cells. Angew. Chem. Int. Ed. 2008, 47, 2402-2406.

27. Bjoerksten, U.; Moser, J.; Graetzel, M., Photoelectrochemical Studies on Nanocrystalline Hematite Films. Chem. Mater. 1994, 6, 858-863.

28. Ou, J. Z.; Rani, R. A.; Ham, M.-H.; Field, M. R.; Zhang, Y.; Zheng, H.; Reece, P.; Zhuiykov, S.; Sriram, S.; Bhaskaran, M., Elevated Temperature Anodized $\mathrm{Nb}_{2} \mathrm{O}_{5}$ : a Photoanode Material with Exceptionally Large Photoconversion Efficiencies. ACS Nano 2012, 6, 4045-4053.

29. Corma, A.; Atienzar, P.; Garcia, H.; Chane-Ching, J.-Y., Hierarchically Mesostructured Doped $\mathrm{CeO}_{2}$ with Potential for Solar-Cell Use. Nat. Mater. 2004, 3, 394-397.

30. Kavan, L.; Vlckova Zivcova, Z.; Zlamalova, M.; Zakeeruddin, S. M.; Grätzel, M., Electron-Selective Layers for Dye-Sensitized Solar Cells Based on $\mathrm{TiO}_{2}$ and $\mathrm{SnO}_{2}$. J. Phys. Chem. C 2020, 124, 6512-6521. 
31. Yang, S.; Kou, H.; Wang, J.; Xue, H.; Han, H., Tunability of the Band Energetics of Nanostructured $\mathrm{SrTiO}_{3}$ Electrodes for Dye-Sensitized Solar Cells. J. Phys. Chem. C 2010, 114, 4245-4249.

32. Shin, S. S.; Kim, J. S.; Suk, J. H.; Lee, K. D.; Kim, D. W.; Park, J. H.; Cho, I. S.; Hong, K. S.; Kim, J. Y., Improved Quantum Efficiency of Highly Efficient Perovskite $\mathrm{BaSnO}_{3}$-Based Dye-Sensitized Solar Cells. ACS Nano 2013, 7, 1027-1035.

33. Tan, B.; Toman, E.; Li, Y.; Wu, Y., Zinc Stannate $\left(\mathrm{Zn}_{2} \mathrm{SnO}_{4}\right)$ Dye-Sensitized Solar Cells. J. Am. Chem. Soc. 2007, 129, 4162-4163.

34. Howarter, J. A.; Youngblood, J. P., Optimization of Silica Silanization by 3aminopropyltriethoxysilane. Langmuir 2006, 22, 11142-11147.

35. Kakiage, K.; Aoyama, Y.; Yano, T.; Oya, K.; Fujisawa, J.-i.; Hanaya, M., HighlyEfficient Dye-Sensitized Solar Cells with Collaborative Sensitization by SilylAnchor and Carboxy-Anchor Dyes. Chem. Commun. 2015, 51, 15894-15897.

36. Nazeeruddin, M. K.; Kay, A.; Rodicio, I.; Humphry-Baker, R.; Müller, E.; Liska, P.; Vlachopoulos, N.; Grätzel, M., Conversion of Light to Electricity by cis-X2bis (2,2'bipyridyl-4,4'-dicarboxylate) Ruthenium (II) Charge-Transfer Sensitizers $\left(\mathrm{X}=\mathrm{Cl}^{-}\right.$, $\mathrm{Br}^{-}, \mathrm{I}^{-}, \mathrm{CN}^{-}$, and $\mathrm{SCN}^{-}$) on Nanocrystalline Titanium Dioxide Electrodes. J. AM. Chem. Soc. 1993, 115, 6382-6390.

37. Chiba, Y.; Islam, A.; Watanabe, Y.; Komiya, R.; Koide, N.; Han, L., Dye-Sensitized Solar Cells with Conversion Efficiency of 11.1\%. Jpn. J. Appl. Phys. 2006, 45, L638.

38. Kalyanasundaram, K., Dye-Sensitized Solar Cells. CRC press: Cleveland, OH, USA, 2010.

39. Gao, F.; Wang, Y.; Shi, D.; Zhang, J.; Wang, M.; Jing, X.; R. Humphry-Baker; Wang, P.; Zakeeruddin, S. M.; Grätzel, M., Enhance the Optical Absorptivity of Nanocrystalline TiO2 Film with High Molar Extinction Coefficient Ruthenium Sensitizers for High Performance Dye-Sensitized Solar Cells. J. Am. Chem. Soc. 2008, $32,10720-10728$. 
40. Brown, P.; Kamat, P. V., Quantum Dot Solar Cells. Electrophoretic Deposition of $\mathrm{CdSe}-\mathrm{C}_{60}$ Composite Films and Capture of Photogenerated Electrons with $\mathrm{n}_{60}$ Cluster Shell. J. Am. Chem. Soc. 2008, 130, 8890-8891.

41. Koumura, N.; Wang, Z.-S.; Miyashita, M.; Uemura, Y.; Sekiguchi, H.; Cui, Y.; Mori, A.; Mori, S.; Hara, K., Substituted Carbazole Dyes for Efficient Molecular Photovoltaics: Long Electron Lifetime and High Open Circuit Voltage Performance. J. Mater. Chem. 2009, 19, 4829-4836.

42. Yu, R. B.; Yu, K. H.; Wei, W.; Xu, X. X.; Qiu, X. M.; Liu, S.; Huang, W.; Tang, G.; Ford, H.; Peng, B., $\mathrm{Nd}_{2} \mathrm{O}_{3}$ Nanoparticles Modified with a Silane-Coupling Agent as a Liquid Laser Medium. Adv. Mater. 2007, 19, 838-842.

43. Hamann, T. W.; Jensen, R. A.; A.B.F. Martinson; Ryswyk, H. V.; Hupp, J. T., Advancing Beyond Current Generation Dye-Sensitized Solar Cells. Energy Environ. Sci. 2008, 1, 66 .

44. Pandit, B.; Luitel, T.; Cummins, D. R.; Thapa, A. K.; Druffel, T.; Zamborini, F. P.; Liu, J., Spectroscopic Investigation of Photoinduced Charge-Transfer Processes in FTO/TiO $2 /$ N719 Photoanodes with and without Covalent Attachment through SilaneBased Linkers. J. Phys. Chem. A 2013, 117, 13513-13523.

45. Luitel, T.; Zamborini, F. P., Covalent Modification of Photoanodes for Stable DyeSensitized Solar Cells. Langmuir 2013, 29, 13582-13894.

46. Diamant, Y.; Chappel, S.; Chen, S. G.; Melamed, O.; Zaban, A., Core-Shell Nanoporous Electrode for Dye Sensitized Solar Cells: the Effect of Shell Characteristics on the Electronic Properties of the Electrode. Coord. Chem. Rev. 2004, 248, 1271-1276.

47. Bandaranayake, K. M. P.; Indika Senevirathna, M. K.; Prasad Weligamuwa, P. M. G. M.; Tennakone, K., Dye-Sensitized Solar Cells Made from Nanocrystalline $\mathrm{TiO}_{2}$ Films Coated with Outer Layers of Different Oxide Materials. Coord. Chem. Rev. 2004, 248, 1277-1281. 
48. Wang, S.; Yu, Z.; Zhang, X.; Ma, D., Nd, N-doped $\mathrm{TiO}_{2}$ Anode Effect on Performance of Dye-Sensitized Solar Cells. Adv. Mater. Rapid Commun. 2013, 7 , $549-552$.

49. Snaith, H. J.; Grätzel, M., Enhanced Charge Mobility in a Molecular Hole Transporter via Addition of Redox Inactive Ionic Dopant: Implication to DyeSensitized Solar Cells. Appl. Phys. Lett. 2006, 89, 262114.

50. Xie, Y.; Huang, N.; Liu, Y.; Sun, W.; Mehnane, H. F.; You, S.; Wang, L.; Liu, W.; Guo, S.; Zhao, X.-Z., Photoelectrodes Modification by N Doping for Dye-Sensitized Solar Cells. Electrochim. Acta 2013, 93, 202-206.

51. https://www.nrel.gov/pv/cell-efficiency.html.

52. Luitel, T.; Fernando, K.; Tatum, B. S.; Alphenaar, B. W.; Zamborini, F. P., Increased Efficiency of Dye-Sensitized Solar Cells by Addition of Rare Earth Oxide Microparticles into a Titania Acceptor. Electrochim. Acta 2016, 211, 918-925.

53. Bhama, S.; Sibakoti, T. R.; Jasinski, J. B.; Zamborini, F. P., Highly Active, Selective, and Recyclable Water-Soluble Glutathione-Stabilized Pd and Pd-Alloy Nanoparticle Catalysts in Biphasic Solvent. ChemCatChem 2020.

54. Sibakoti, T.; Jasinski, J. B.; Nantz, M. H.; Zamborini, F., Iodine Activation: A General Method for Catalytic Enhancement of Thiolate Monolayer-Protected Metal Clusters. Nanoscale 2020.

55. Sibakoti, T. R.; Stinger, C. R.; Adhihetty, P. K.; Zamborini, F. P.; Nantz, M. H., Tunable Aminooxy-Functionalized Monolayer-Protected Gold Clusters for Nonpolar and Aqueous Oximation Reactions. Par. Par. Sys. Char. 2019, 36, 1900093.

56. Pattadar, D. K.; Zamborini, F. P., Size Stability Study of Catalytically Active Sub-2 nm Diameter Gold Nanoparticles Synthesized with Weak Stabilizers. J. Am. Chem. Soc. 2018, 140, 14126-14133.

57. Sharma, J. N.; Pattadar, D. K.; Mainali, B. P.; Zamborini, F. P., Size Determination of Metal Nanoparticles Based on Electrochemically Measured Surface-Area-toVolume Ratios. Anal. Chem. 2018, 90, 9308-9314. 
58. Pattadar, D. K.; Mainali, B. P.; Jasinski, J. B.; Zamborini, F. P., Electrooxidation, Size Stability, and Electrocatalytic Activity of $0.9 \mathrm{~nm}$ Diameter Gold Nanoclusters Coated with a Weak Stabilizer. ChemElectroChem 2020, 7, 800-809.

59. Pattadar, D. K.; Sharma, J. N.; Mainali, B. P.; Zamborini, F. P., Anodic Stripping Electrochemical Analysis of Metal Nanoparticles. Curr. Opi. Electrochem. 2019, 13, $147-156$.

60. Pattadar, D. K.; Zamborini, F. P., Halide-Dependent Dealloying of $\mathrm{Cu}_{\mathrm{x}} / \mathrm{Au}_{\mathrm{y}}$ Core/Shell Nanoparticles for Composition Analysis by Anodic Stripping Voltammetry. J. Phys. Chem. C 2019, 123, 9496-9505.

61. Pattadar, D. K.; Sharma, J. N.; Mainali, B. P.; Zamborini, F. P., Impact of the Assembly Method on the Surface Area-to-Volume Ratio and Electrochemical Oxidation Potential of Metal Nanospheres. The Journal of Physical Chemistry $C$ 2019, 123, 24304-24312.

62. Dong, Q.; Fang, Y.; Shao, Y.; Mulligan, P.; Qiu, J.; Cao, L.; Huang, J., Electron-Hole Diffusion Lengths $>175 \mu \mathrm{m}$ in Solution-Grown $\mathrm{CH}_{3} \mathrm{NH}_{3} \mathrm{PbI}_{3}$ Single Crystals. Science 2015, 347, 967-970.

63. Wu, K.; Bera, A.; Ma, C.; Du, Y.; Yang, Y.; Li, L.; Wu, T., Temperature-Dependent Excitonic Photoluminescence of Hybrid Organometal Halide Perovskite Films. Phys. Chem. Chem. Phys. 2014, 16, 22476-22481.

64. De Wolf, S.; Holovsky, J.; Moon, S.-J.; Löper, P.; Niesen, B.; Ledinsky, M.; Haug, F.-J.; Yum, J.-H.; Ballif, C., Organometallic Halide Perovskites: Sharp Optical Absorption Edge and its Relation to Photovoltaic Performance. J. Phys. Chem. Lett. 2014, 5, 1035-1039.

65. Löper, P.; Stuckelberger, M.; Niesen, B.; Werner, J. r. m.; Filipič, M.; Moon, S.-J.; Yum, J.-H.; Topič, M.; De Wolf, S.; Ballif, C., Complex Refractive Index Spectra of $\mathrm{CH}_{3} \mathrm{NH}_{3} \mathrm{PbI}_{3}$ Perovskite Thin Films Determined by Spectroscopic Ellipsometry and Spectrophotometry. J. Phys. Chem. Lett. 2015, 6, 66-71. 
66. Yang, Y.; Yan, Y.; Yang, M.; Choi, S.; Zhu, K.; Luther, J. M.; Beard, M. C., Low Surface Recombination Velocity in Solution-Grown $\mathrm{CH}_{3} \mathrm{NH}_{3} \mathrm{PbBr}_{3}$ Perovskite Single Crystal. Nat. Commun. 2015, 6, 7961.

67. Ziang, X.; Shifeng, L.; Laixiang, Q.; Shuping, P.; Wei, W.; Yu, Y.; Li, Y.; Zhijian, C.; Shufeng, W.; Honglin, D., Refractive Index and Extinction Coefficient of $\mathrm{CH}_{3} \mathrm{NH}_{3} \mathrm{PbI}_{3}$ Studied by Spectroscopic Ellipsometry. Opt. Mater. Express 2015, 5 , $29-43$.

68. Kim, Y. H.; Cho, H.; Heo, J. H.; Kim, T. S.; Myoung, N.; Lee, C. L.; Im, S. H.; Lee, T. W., Multicolored Organic/Inorganic Hybrid Perovskite Light-Emitting Diodes. Adv. Mater. 2015, 27, 1248-1254.

69. Xing, G.; Mathews, N.; Lim, S. S.; Yantara, N.; Liu, X.; Sabba, D.; Grätzel, M.; Mhaisalkar, S.; Sum, T. C., Low-Temperature Solution-Processed WavelengthTunable Perovskites for Lasing. Nat. Mater. 2014, 13, 476-480.

70. Noh, J. H.; Im, S. H.; Heo, J. H.; Mandal, T. N.; Seok, S. I., Chemical Management for Colorful, Efficient, and Stable Inorganic-Organic Hybrid Nanostructured Solar Cells. Nano lett. 2013, 13, 1764-1769.

71. Colella, S.; Mosconi, E.; Fedeli, P.; Listorti, A.; Gazza, F.; Orlandi, F.; Ferro, P.; Besagni, T.; Rizzo, A.; Calestani, G., $\mathrm{MAPbI}_{3-\mathrm{x}} \mathrm{Cl}_{\mathrm{x}}$ Mixed Halide Perovskite for Hybrid Solar Cells: The Role of Chloride as Dopant on the Transport and Structural Properties. Chem. Mater. 2013, 25, 4613-4618.

72. Yang, W. S.; Noh, J. H.; Jeon, N. J.; Kim, Y. C.; Ryu, S.; Seo, J.; Seok, S. I., HighPerformance Photovoltaic Perovskite Layers Fabricated through Intramolecular Exchange. Science 2015, 348, 1234-1237.

73. Yang, W. S.; Park, B.-W.; Jung, E. H.; Jeon, N. J.; Kim, Y. C.; Lee, D. U.; Shin, S. S.; Seo, J.; Kim, E. K.; Noh, J. H., Iodide Management in Formamidinium-LeadHalide-Based Perovskite Layers for Efficient Solar Cells. Science 2017, 356, 13761379. 
74. Dou, L.; Yang, Y. M.; You, J.; Hong, Z.; Chang, W. H.; Li, G.; Yang, Y., SolutionProcessed Hybrid Perovskite Photodetectors with High Detectivity. Nat. Commun. 2014, 5, 5404.

75. Zhu, H.; Fu, Y.; Meng, F.; Wu, X.; Gong, Z.; Ding, Q.; Gustafsson, M. V.; Trinh, M. T.; Jin, S.; Zhu, X., Lead Halide Perovskite Nanowire Lasers with Low Lasing Thresholds and High Quality Factors. Nat. Mater. 2015, 14, 636.

76. Deschler, F.; Price, M.; Pathak, S.; Klintberg, L. E.; Jarausch, D.-D.; Higler, R.; Hüttner, S.; Leijtens, T.; Stranks, S. D.; Snaith, H. J., High Photoluminescence Efficiency and Optically Pumped Lasing in Solution-Processed Mixed Halide Perovskite Semiconductors. J. Phys. Chem. Lett. 2014, 5, 1421-1426.

77. Zhu, X.; Lee, J.; Lu, W. D., Iodine Vacancy Redistribution in Organic-Inorganic Halide Perovskite Films and Resistive Switching Effects. Adv. Mater. 2017, 29, 1700527.

78. Xiao, Z.; Huang, J., Energy-Efficient Hybrid Perovskite Memristors and Synaptic Devices. Adv. Electron. Mater. 2016, 2, 1600100.

79. Yan, K.; Peng, M.; Yu, X.; Cai, X.; Chen, S.; Hu, H.; Chen, B.; Gao, X.; Dong, B.; Zou, D., High-Performance Perovskite Memristor Based on Methyl Ammonium Lead Halides. J. Mater. Chem. C 2016, 4, 1375-1381.

80. Jacobsson, T. J.; Correa-Baena, J.-P.; Halvani Anaraki, E.; Philippe, B.; Stranks, S. D.; Bouduban, M. E.; Tress, W.; Schenk, K.; Teuscher, J. 1.; Moser, J.-E., Unreacted $\mathrm{PbI}_{2}$ as a Double-Edged Sword for Enhancing the Performance of Perovskite Solar Cells. J. Am. Chem. Soc. 2016, 138, 10331-10343.

81. Cao, J.; Jing, X.; Yan, J.; Hu, C.; Chen, R.; Yin, J.; Li, J.; Zheng, N., Identifying the Molecular Structures of Intermediates for Optimizing the Fabrication of HighQuality Perovskite Films. J. Am. Chem. Soc. 2016, 138, 9919-9926.

82. Buin, A.; Pietsch, P.; Xu, J.; Voznyy, O.; Ip, A. H.; Comin, R.; Sargent, E. H., Materials Processing Routes to Trap-Free Halide Perovskites. Nano Lett. 2014, 14, 6281-6286. 
83. Eames, C.; Frost, J. M.; Barnes, P. R.; O’regan, B. C.; Walsh, A.; Islam, M. S., Ionic Transport in Hybrid Lead Iodide Perovskite Solar Cells. Nat. Commun. 2015, 6, 7497.

84. Kim, H.-S.; Kim, S. K.; Kim, B. J.; Shin, K.-S.; Gupta, M. K.; Jung, H. S.; Kim, S.W.; Park, N.-G., Ferroelectric Polarization in $\mathrm{CH}_{3} \mathrm{NH}_{3} \mathrm{PbI}_{3}$ Perovskite. J. Phys. Chem. Lett. 2015, 6, 1729-1735.

85. Xiao, J.; Chang, J.; Li, B.; Isikgor, F. H.; Wang, D.; Fan, Z.; Lin, Z.; Ouyang, J.; Zeng, K.; Chen, J., Room Temperature Ferroelectricity of Hybrid Organic-Inorganic Perovskites with Mixed Iodine and Bromine. J. Mater. Chem. A 2018, 6, 9665-9676.

86. Zhang, H.-Y.; Song, X.-J.; Chen, X.-G.; Zhang, Z.-X.; You, Y.-M.; Tang, Y.-Y.; Xiong, R.-G., Observation of Vortex Domains in a Two-Dimensional Lead Iodide Perovskite Ferroelectric. J. Am. Chem. Soc. 2020, 142, 4925-4931.

87. Liao, W.-Q.; Zhang, Y.; Hu, C.-L.; Mao, J.-G.; Ye, H.-Y.; Li, P.-F.; Huang, S. D.; Xiong, R.-G., A Lead-Halide Perovskite Molecular Ferroelectric Semiconductor. Nat. Commun. 2015, 6, 1-7.

88. Chen, P.-Y.; Qi, J.; Klug, M. T.; Dang, X.; Hammond, P. T.; Belcher, A. M., Environmentally Responsible Fabrication of Efficient Perovskite Solar Cells from Recycled Car Batteries. Energy Environ. Sci. 2014, 7, 3659-3665.

89. Babayigit, A.; Ethirajan, A.; Muller, M.; Conings, B., Toxicity of Organometal Halide Perovskite Solar Cells. Nat. Mater. 2016, 15, 247.

90. Niu, G.; Guo, X.; Wang, L., Review of Recent Progress in Chemical Stability of Perovskite Solar Cells. J. Mater. Chem. A 2015, 3, 8970-8980.

91. Leijtens, T.; Eperon, G. E.; Noel, N. K.; Habisreutinger, S. N.; Petrozza, A.; Snaith, H. J., Stability of Metal Halide Perovskite Solar Cells. Adv. Energy Mater. 2015, 5 , 1500963.

92. Jeon, N. J.; Noh, J. H.; Yang, W. S.; Kim, Y. C.; Ryu, S.; Seo, J.; Seok, S. I., Compositional Engineering of Perovskite Materials for High-Performance Solar Cells. Nature 2015, 517, 476. 
93. Liu, P.; Wang, W.; Liu, S.; Yang, H.; Shao, Z., Fundamental Understanding of Photocurrent Hysteresis in Perovskite Solar Cells. Adv. Energy Mater. 2019, 9, 1803017.

94. Wei, J.; Zhao, Y.; Li, H.; Li, G.; Pan, J.; Xu, D.; Zhao, Q.; Yu, D., Hysteresis Analysis Based on the Ferroelectric Effect in Hybrid Perovskite Solar Cells. J. Phys. Chem. Lett. 2014, 5, 3937-3945.

95. Jacobs, D. A.; Wu, Y.; Shen, H.; Barugkin, C.; Beck, F. J.; White, T. P.; Weber, K.; Catchpole, K. R., Hysteresis Phenomena in Perovskite Solar Cells: the Many and Varied Effects of Ionic Accumulation. Phys. Chem. Chem. Phys. 2017, 19, 30943103.

96. Li, C.; Tscheuschner, S.; Paulus, F.; Hopkinson, P. E.; Kießling, J.; Köhler, A.; Vaynzof, Y.; Huettner, S., Iodine Migration and its Effect on Hysteresis in Perovskite Solar Cells. Adv. Mater. 2016, 28, 2446-2454.

97. Yuan, Y.; Chae, J.; Shao, Y.; Wang, Q.; Xiao, Z.; Centrone, A.; Huang, J., Photovoltaic Switching Mechanism in Lateral Structure Hybrid Perovskite Solar Cells. Adv. Energy Mater. 2015, 5, 1500615.

98. Jiang, C.-S.; Yang, M.; Zhou, Y.; To, B.; Nanayakkara, S. U.; Luther, J. M.; Zhou, W.; Berry, J. J.; Van De Lagemaat, J.; Padture, N. P., Carrier Separation and Transport in Perovskite Solar Cells Studied by Nanometre-Scale Profiling of Electrical Potential. Nat. Commun. 2015, 6, 8397.

99. Khadka, D. B.; Shirai, Y.; Yanagida, M.; Miyano, K., Unraveling the Impacts Induced by Organic and Inorganic Hole Transport Layers in Inverted Halide Perovskite Solar Cells. ACS Appl. Mater. Interfaces 2019, 11, 7055-7065.

100. Smith, I. C.; Hoke, E. T.; Solis-Ibarra, D.; McGehee, M. D.; Karunadasa, H. I., A Layered Hybrid Perovskite Solar-Cell Absorber with Enhanced Moisture Stability. Angew. Chem. Int. Ed. 2014, 53, 11232-11235.

101. Cao, D. H.; Stoumpos, C. C.; Farha, O. K.; Hupp, J. T.; Kanatzidis, M. G., 2D Homologous Perovskites as Light-Absorbing Materials for Solar Cell Applications. J. Am. Chem. Soc 2015, 137, 7843-750. 
102. Stoumpos, C. C.; Cao, D. H.; Clark, D. J.; Young, J.; Rondinelli, J. M.; Jang, J. I.; Hupp, J. T.; Kanatzidis, M. G., Ruddlesden-Popper Hybrid Lead Iodide Perovskite 2D Homologous Semiconductors. Chem. Mater. 2016, 28, 2852-2867.

103. Lanty, G.; Jemli, K.; Wei, Y.; Leymarie, J.; Even, J.; Lauret, J.-S.; Deleporte, E., Room-Temperature Optical Tunability and Inhomogeneous Broadening in 2DLayered Organic-Inorganic Perovskite Pseudobinary Alloys. J. Phys. Chem. Lett. 2014, 5, 3958-3963.

104. Borriello, I.; Cantele, G.; Ninno, D., Ab Initio Investigation of Hybrid OrganicInorganic Perovskites Based on Tin Halides. Phys. Rev. B 2008, 77, 235214.

105. Wang, P.; Zakeeruddin, S. M.; Comte, P.; Charvet, R.; Humphry-Baker, R.; Grätzel, M., Enhance the Performance of Dye-Sensitized Solar Cells by Co-Grafting Amphiphilic Sensitizer and Hexadecylmalonic Acid on $\mathrm{TiO}_{2}$ Nanocrystals. J. Phys. Chem. B 2003, 107, 14336-14341.

106. Ito, S.; Chen, P.; Comte, P.; Nazeeruddin, M. K.; Liska, P.; Péchy, P.; Grätzel, M., Fabrication of Screen-Printing Pastes from $\mathrm{TiO}_{2}$ Powders for Dye-Sensitised Solar Cells. Progress in Photovoltaics: Research and Applications 2007, 15, 603-612.

107. Juarez-Perez, E. J.; Sanchez, R. S.; Badia, L.; Garcia-Belmonte, G.; Kang, Y. S.; Mora-Sero, I.; Bisquert, J., Photoinduced Giant Dielectric Constant in Lead Halide Perovskite Solar Cells. J. Phys. Chem. Lett. 2014, 5, 2390-2394.

108. ASTM, Standard Test Methods for Determining Average Grain Size; https://compass.astm.org/download/E112.17793.pdf.

109. Lewis, N. S.; Crabtree, G. G., Basic Research Needs for Solar Energy Utilization: Report of the Basic Energy Sciences Workshop on Solar Energy Utilization. April 18-21, 2005.

110. Lewis, N. S.; Nocera, D. G., Powering the Planet- Chemical Challenges in Solar Energy Utilization. Proc. Natl. Acad. Sci. U.S.A. 2006, 103, 15729-15735.

111. Tsubomura, H.; Matsumura, M.; Nomura, Y.; Amamiya, T., Dye Sensitised Zinc Oxide: Aqueous Electrolyte: Platinum Photocell. Nature 1976, 261, 402-403. 
112. Grätzel, M., Dye-Sensitized Solar Cells. J. Photochem. Photobiol. C: Photochem. Rev. 2003, 4, 145-153.

113. Grätzel, M.; O’Regan, B., A Low-Cost, High-Efficiency Solar Cell Based on DyeSensitized Colloidal $\mathrm{TiO}_{2}$ Films. Nature 1991, 353, 737-740.

114. Grätzel, M., Photoelectrochemical Cells. Nature 2001, 414, 338-344.

115. Berglund, S. P.; Hoang, S.; Minter, R. L.; Fullon, R. R.; Mullins, C. B., Investigation of 35 Elements as Single Metal Oxides, Mixed Metal Oxides, or Dopants for Titanium Dioxide for Dye-Sensitized Solar Cells. J. Phys. Chem. C 2013, 117, 25248-25258.

116. Gomez, V.; Balu, A. M.; Serrano-Ruiz, J. C.; Irusta, S.; Dionysiou, D. D.; Luque, R.; Santamaría, J., Microwave-Assisted Mild-Temperature Preparation of NeodymiumDoped Titania for the Improved Photodegradation of Water Contaminants. Appl. Catal. A 2012, 441-442, 47-53.

117. Zhao, B.; Wang, J.; Li, H.; Wang, H.; Jia, X.; Su, P., The Influence of Yttrium Dopant on the Properties of Anatase Nanoparticles and the Performance of Dye-Sensitized Solar Cells. Phys. Chem. Chem. Phys. 2015, 17, 14836-14842.

118. Zalas, M., Gadolinium-Modified Titanium Oxide Materials for Photoenergy Applications: A Review. J. Rare Earths 2014, 32, 487-495.

119. Khan, A. A.; Johirul, J.; Ansari, S. G.; Fouad, H.; Ansari, Z. A., Effect of Neodymium on the Photoconversion Efficiency of $\mathrm{TiO}_{2}$ Based Dye Sensitized Solar Cells. $J$. Mater. Sci.: Mater. Electron. 2014, 26, 1737-1742.

120. Bisquert, J., Chemical Capacitance of Nanostructured Semiconductors: its Origin and Significance for Nanocomposite Solar Cells. Phys. Chem. Chem. Phys. 2003, 5, 5360.

121. Shogh, S.; Mohammadpour, R.; Zad, A. I.; Taghavinia, N., Improved Photovoltaic Performance of Nanostructured Solar Cells by Neodymium-Doped $\mathrm{TiO}_{2}$ Photoelectrode. Mater. Lett. 2015, 159, 273-275. 
122. Cavallo, C.; Salleo, A.; Gozzi, D.; Pascasio, F. D.; Quaranta, S.; Panetta, R.; Latini, A., Solid Solutions of Rare Earth Cations in Mesoporous Anatase Beads and their Performances in Dye-Sensitized Solar Cells. Sci. Rep. 2015, 5, 16785.

123. Li, W.; Wang, Y.; Lin, H.; Shah, S. I.; Huang, C. P.; Doren, D. J.; Rykov, S. A.; Chen, J. G.; Barteau, M. A., Band Gap Tailoring of $\mathrm{Nd}^{3+}$-doped $\mathrm{TiO}_{2}$ Nanoparticles. Appl. Phys. Lett. 2003, 83, 4143-4145.

124. Chen, S.; Lin, J.; Wu, J., Improving Photoelectrical Performance of Dye Sensitized Solar Cells by Doping $\mathrm{Y}_{2} \mathrm{O}_{3}: \mathrm{Tb}^{3+}$ Nanorods. J. Mater. Sci.: Mater. Electron. 2014, $25,2060-2065$.

125. Zhang, J.; Peng, W.; Chen, Z.; Chen, H.; Han, L., Effect of Cerium Doping in the $\mathrm{TiO}_{2}$ Photoanode on the Electron Transport of Dye-Sensitized Solar Cells. J. Phys. Chem. C 2012, 116, 19182-19190.

126. Yao, Q.; Liu, J.; Peng, Q.; Wang, X.; Li, Y., Nd-Doped $\mathrm{TiO}_{2}$ Nanorods: Preparation and Application in Dye-Sensitized Solar Cells. Chem. Asian J. 2006, 1, 737-41.

127. Luitel, T.; Fernando, K.; Tatuma, B. S.; Alphenaar, B. W.; Zamborini, F. P., Increased Efficiency of Dye-Sensitized Solar Cells by Addition of Rare-Earth Oxide Microparticles into a Titania Acceptor. Electrochim. Acta 2016, 211, 918-925.

128. Hafez, H.; Saif, M.; Abdel-Mottaleb, M. S. A., Down-Converting Lanthanide Doped $\mathrm{TiO}_{2}$ Photoelectrodes for Efficiency Enhancement of Dye-Sensitized Solar Cells. $J$. Power Sources 2011, 196, 5792-5796.

129. Yu, J.; Yang, Y.; Fan, R.; Zhang, H.; Li, L.; Wei, L.; Shi, Y.; Pan, K.; Fu, H., Er ${ }^{3+}$ and $\mathrm{Yb}^{3+} \mathrm{Co}$-Doped $\mathrm{TiO}_{2-\mathrm{x}} \mathrm{F}_{\mathrm{x}}$ Up-Conversion Luminescence Powder as a Light Scattering Layer with Enhanced Performance in Dye Sensitized Solar Cells. J. Power Sources 2013, 243, 436-443.

130. Shan, G. B.; Assaaoudi, H.; Demopoulos, G. P., Enhanced Performance of DyeSensitized Solar Cells By Utilization of an External, Bifunctional Layer Consisting of Uniform beta-NaYF $4: \mathrm{Er}^{3+} / \mathrm{Yb}^{3+}$ Nanoplatelets. ACS Appl. Mater. Interfaces 2011, $3,3239-3243$. 
131. Wang, Q.; Moser, J. E.; Grätzel, M., Electrochemical Impedance Spectroscopic Analysis of Dye-Sensitized Solar Cells. J. Phys. Chem. B 2005, 109, 14945-14953.

132. Yang, W.; Li, X.; Chi, D.; Zhang, H.; Liu, X., Lanthanide-doped upconversion materials: emerging applications for photovoltaics and photocatalysis. Nanotechnology 2014, 25, 482001.

133. Li, F.; Gu, Y., Improvement of performance of dye-sensitized solar cells by doping Er2O3 into TiO2 electrodes. Mater. Sci. Semicond. Process. 2012, 15, 11-14.

134. Kharel, P. L.; Cuillier, P. M.; Fernando, K.; Zamborini, F. P.; Alphenaar, B. W., Effect of Rare-Earth Metal Oxide Nanoparticles on the Conductivity of Nanocrystalline Titanium Dioxide: An Electrical and Electrochemical Approach. The Journal of Physical Chemistry C 2018, 122, 15090-15096.

135. Kumar, S. G.; Devi, L. G., Review on Modified $\mathrm{TiO}_{2}$ Photocatalysis Under UV/Visible Light: Selected Results and Related Mechanisms on Interfacial Charge Carrier Transfer Dynamics. J. Phys. Chem. A 2011, 115, 13211-13241.

136. Macwan, D. P.; Dave, P. N.; Chaturvedi, S., A Review on Nano-TiO 2 Sol-Gel Type Syntheses and its Applications. J. Mater. Sci. 2011, 46, 3669-3686.

137. Bisquert, J.; Fabregat-Santiago, F.; Mora-Seró, I.; Garcia-Belmonte, G.; Barea, E. M.; Palomares, E., A Review of Recent Results on Electrochemical Determination of the Density of Electronic States of Nanostructured Metal-Oxide Semiconductors and Organic Hole Conductors. Inorg. Chim. Acta 2008, 361, 684-698.

138. Berger, T.; Monllor-Satoca, D.; Jankulovska, M.; Lana-Villarreal, T.; Gómez, R., The Electrochemistry of nanostructured titanium dioxide electrodes. ChemPhysChem 2012, 13, 2824-2875.

139. Cheng, P.; Zheng, M.; Jin, Y.; Huang, Q.; Gu, M., Preparation and Characterization of Silica-Doped Titania Photocatalyst Through Sol-Gel Method. Mater. Lett. 2003, 57, 2989-2994.

140. Yin, S.; Zhang, Q.; Saito, F.; Sato., T., Preparation of Visible Light-Activated Titania Photocatalyst by Mechanochemical Method. Chem. Lett. 2003, 32, 358-359. 
141. Beydoun, D.; Amal, R.; Low, G.; McEvoy, S., Role of Nanoparticles in Photocatalysis. J. Nanopart. Res. 1999, 1, 439-458.

142. Stathatos, E.; Tsiourvas, D.; Lianos, P., Titanium dioxide films made from reverse micelles and their use for the photocatalytic degradation of adsorbed dyes. Colloids Surf., A 1999, 149, 49-56.

143. Li, Q.; Mahendra, S.; Lyon, D. Y.; Brunet, L.; Liga, M. V.; Li, D.; Alvarez, P. J., Antimicrobial nanomaterials for Water Disinfection and Microbial Control: Potential Applications and Implications. Water Res. 2008, 42, 4591-4602.

144. Comparelli, R.; Fanizza, E.; Curri, M. L.; Cozzoli, P. D.; Mascolo, G.; Passino, R.; Agostiano, A., Photocatalytic degradation of azo dyes by organic-capped anatase $\mathrm{TiO}_{2}$ nanocrystals immobilized onto substrates. Appl. Catal., B 2005, 55, 81-91.

145. Fabregat-Santiago, F.; Mora-Seró, I.; Garcia-Belmonte, G.; Bisquert, J., Cyclic voltammetry studies of nanoporous semiconductors. capacitive and reactive properties of nanocrystalline $\mathrm{TiO}_{2}$ electrodes in aqueous electrolyte. J. Phys. Chem. B 2003, 107, 758-768.

146. Djenizian, T.; Hanzu, I.; Knauth, P., Nanostructured Negative Electrodes Based on Titania for Li-ion microbatteries. J. Mater. Chem. 2011, 21, 9925-9937.

147. Liu, M.; Johnston, M. B.; Snaith, H. J., Efficient Planar Heterojunction Perovskite Solar Cells by Vapour Deposition. Nature 2013, 501, 395-398.

148. Lee, M. M.; Teuscher, J.; Miyasaka, T.; Murakami, T. N.; Snaith, H. J., Efficient Hybrid Solar Cells Based on Meso-Superstructured Organometal Halide Perovskites. Science 2012, 338.

149. Burschka, J.; Pellet, N.; Moon, S. J.; Humphry-Baker, R.; Gao, P.; Nazeeruddin, M. K.; Grätzel, M., Sequential Deposition as a Route to High-Performance

Perovskite-Sensitized Solar Cells. Nature 2013, 499, 316-320.

150. Chen, Q.; Zhou, H.; Hong, Z.; Luo, S.; Duan, H.-S.; Wang, H.-H.; Liu, Y.; Li, G.; Yang, Y., Planar Heterojunction Perovskite Solar Cells via Vapor-Assisted Solution Process. J. Am. Chem. Soc. 2014, 136, 622-625. 
151. Kavan, L.; Gräizel, M.; Rathouský, J.; Zukalb, A., Nanocrystalline $\mathrm{TiO}_{2}$ (Anatase) Electrodes: Surface Morphology, Adsorption, and Electrochemical Properties. $J$. Electrochem. Soc. 1996, 143, 394-400.

152. Wang, P.; Zakeeruddin, S. M.; Comte, P.; Charvet, R.; Humphry-Baker, R.; Grätzel, M., Enhance the Performance of Dye-Sensitized Solar Cells by Co-Grafting Amphiphilic Sensitizer and Hexadecylmalonic Acid on $\mathrm{TiO}_{2}$ Nanocrystals. J. Phys. Chem. B 2003, 107, 14336-14341.

153. Bandaranayake, K. M. P.; Senevirathna, M. I.; Weligamuwa, P. P.; Tennakone, K., Dye-Sensitized Solar Cells Made from Nanocrystalline $\mathrm{TiO}_{2}$ Films Coated with Outer Layers of Different Oxide Materials. Coord. Chem. Rev. 2004, 248, 1277-1281.

154. Grätzel, M., Conversion of Sunlight to Electric Power by Nanocrystalline DyeSensitized Solar Cells. J. Photochem. Photobio. A Chem. 2004, 164, 3-14.

155. Li, B.; Wang, L.; Kang, B.; Wang, P.; Qiu, Y., Review of Recent Progress in SolidState Dye-Sensitized Solar Cells. Sol. Energy Mater. Sol. Cells 2006, 90, 549-573.

156. Günes, S.; Sariciftci, N. S., Hybrid Solar Cells. Inorganica Chim. Acta 2008, 361, 581-588.

157. Mora-Sero, I.; Gimenez, S.; Fabregat-Santiago, F.; Gomez, R.; Shen, Q.; Toyoda, T.; Bisquert, J., Recombination in Quantum Dot Sensitized Solar Cells. Acc. Chem. Res. 2009, 42, 1848-1857.

158. Bertoluzzi, L.; Herraiz-Cardona, I.; Gottesman, R.; Zaban, A.; Bisquert, J., Relaxation of Electron Carriers in the Density of States of Nanocrystalline $\mathrm{TiO}_{2}$. J. Phys. Chem. Lett. 2014, 5, 689-694.

159. Snaith, H. J.; Schmidt-Mende, L., Advances in Liquid-Electrolyte and Solid-State Dye-Sensitized Solar Cells. Adv. Mater. 2007, 19, 3187-3200.

160. Jankulovska, M.; Berger, T.; Wong, S. S.; Gómez, R.; Lana-Villarreal, T., Trap States in $\mathrm{TiO}_{2}$ Films Made of Nanowires, Nanotubes Or Nanoparticles: An Electrochemical Study. ChemPhysChem 2012, 13, 3008-3017. 
161. Tang, H.; K. Prasad; R. Sanjinès; Schmid, P. E.; Lévy, F., Electrical and Optical Properties of $\mathrm{TiO}_{2}$ Anatase Thin Films. J. Appl. Phys. 1994, 75, 2042-2047.

162. Schneider, J.; Matsuoka, M.; Takeuchi, M.; Zhang, J.; Horiuchi, Y.; Anpo, M.; Bahnemann, D. W., Understanding $\mathrm{TiO}_{2}$ Photocatalysis: Mechanisms and Materials. Chem. Rev. 2014, 114, 9919-9986.

163. Xu, A.-W.; Gao, Y.; Liu, H.-Q., The Preparation, Characterization, and their Photocatalytic Activities of Rare-Earth-Doped $\mathrm{TiO}_{2}$ Nanoparticles. J. Catal. 2002, 207, 151-157.

164. Bhethanabotla, V. C.; Russell, D. R.; Kuhn, J. N., Assessment of Mechanisms for Enhanced Performance of $\mathrm{Yb} / \mathrm{Er} /$ Titania Photocatalysts for Organic Degradation: Role of Rare Earth Elements in the Titania Phase. Appl. Catal., B 2017, 202, 156164.

165. Mandari, K. K.; Police, A. K. R.; Do, J. Y.; Kang, M.; Byon, C., Rare Earth Metal Gd Influenced Defect Sites in $\mathrm{N}_{\text {Doped }} \mathrm{TiO}_{2}$ : Defect Mediated Improved Charge Transfer for Enhanced Photocatalytic Hydrogen Production. Int. J. Hydrogen Energy 2018, 43, 2073-2082.

166. Shi, J.; Zou, Y.; Ma, D., Ce-Doped Titania Nanoparticles: The Effects of Doped Amount and Calcination Temperature on Photocatalytic Activity. IOP Conf.: Mater. Sci. Eng. 2017, 167, 012039.

167. Luitel, T.; Fernando, K.; Tatum, B. S.; Alphenaar, B. W.; Zamborini, F. P., Increased Efficiency of Dye-Sensitized Solar Cells by Addition of Rare-Earth Oxide Microparticles into a Titania Acceptor. Electrochim. Acta 2016, 211, 918-925.

168. Wang, S.; Yu, Z.; Zhang, X.; Ma, D., Nd, N-Doped $\mathrm{TiO}_{2}$ Anode Effect on Performance of Dye-Sensitized Solar Cells Optoelectron. Adv. Mater. Rapid Commun. 2013, 7, 549-552.

169. Yao, Q.; Liu, J.; Peng, Q.; Wang, X.; Li, Y., Nd-doped $\mathrm{TiO}_{2}$ Nanorods: Preparation and Application in Dye-Sensitized Solar Cells. Chem. Asian J. 2006, 1, 737-741. 
170. Kharel, P. L.; Zamborini, F. P.; Alphenaar, B. W., Enhancing the Photovoltaic Performance of Dye-Sensitized Solar Cells with Rare-Earth Metal Oxide Nanoparticles. J. Electrochem. Soc. 2018, 165, H52-H56.

171. Kucur, E.; Bucking, W.; Giernoth, R.; Nann, T., Determination of Defect States in Semiconductor Nanocrystals by Cyclic Voltammetry. J. Phys. Chem. B 2005, 109, 20355-20360.

172. Fabregat-Santiago, F.; Randriamahazaka, H.; Zaban, A.; Garcia-Canadas, J.; GarciaBelmonte, G.; Bisquert, J., Chemical Capacitance of Nanoporous-Nanocrystalline $\mathrm{TiO}_{2}$ in a Room Temperature Ionic Liquid. Phys. Chem. Chem. Phys. 2006, 8, 18271833.

173. Wang, H.; He, J.; Boschloo, G.; Lindström, H.; Hagfeldt, A.; Lindquist, S.-E., Electrochemical Investigation of Traps in a Nanostructured $\mathrm{TiO}_{2}$ Film. J. Phys. Chem. B 2001, 105, 2529-2533.

174. Chmiola, J.; Yushin, G.; Dash, R.; Gogotsi, Y., Effect of Pore Size and Surface Area of Carbide Derived Carbons on Specific Capacitance. J. Power Sources 2006, 158, 765-772.

175. Beranek, R., (Photo)Electrochemical Methods for the Determination of The Band Edge Positions of $\mathrm{TiO}_{2}$-Based Nanomaterials. Adv. Phys. Chem. 2011, 2011, 1-20.

176. Gelderman, K.; Lee, L.; Donne, S. W., Flat-Band Potential of a Semiconductor Using the Mott-Schottky Equation. J. Chem. Educ. 2007, 84, 685.

177. Sze, S. M., Physics of Semiconductor Devices. John Wiley and Sons: Hoboken, NJ, 2007.

178. Brotherton, S. D., Introduction to Thin Film Transistors. In Semiconductor Device Physics for TFTs, Springer: Heidelberg, Germany, 2013; pp 9-44.

179. Zhang, Z.; Yates Jr, J. T., Band Bending in Semiconductors: Chemical and Physical Consequences at Surfaces and Interfaces. Chem. Rev. 2012, 112, 5520-51.

180. Mousavi, M.; Habibi-Yangjeh, A.; Abitorabi, M., Fabrication of novel magnetically separable nanocomposites using graphitic carbon nitride, silver phosphate and silver 
chloride and their applications in photocatalytic removal of different pollutants using visible-light irradiation. Journal of colloid and interface science 2016, 480, 218-231.

181. Habibi-Yangjeh, A.; Shekofteh-Gohari, M., Novel magnetic Fe3O4/ZnO/NiWO4 nanocomposites: Enhanced visible-light photocatalytic performance through pn heterojunctions. Separation and Purification Technology 2017, 184, 334-346.

182. Prokofiev, A. V.; Shelykh, A. I.; Melekh, B. T., Periodicity in the band gap variation of $\mathrm{Ln}_{2} \mathrm{X}_{3}(\mathrm{X}=\mathrm{O}, \mathrm{S}, \mathrm{Se})$ in the lanthanide series. J. Alloys Compd. 1996, 242, 41-44.

183. Fanciulli, M.; Scarel, G., Rare Earth Oxide Thin Films. Springer: Berlin, Germany, 2007.

184. Robertson, J.; Falabretti, B., Band Offsets of High K Gate Oxides on III-V Semiconductors. J. Appl. Phys. 2006, 100, 014111.

185. Wu, H.; Wang, L.-S., Electronic Structure of Titanium Oxide Clusters- $\mathrm{TiO}_{\mathrm{y}}(\mathrm{y}=1-3)$ and $\left(\mathrm{TiO}_{2}\right)_{\mathrm{n}}(\mathrm{n}=1-4)$. J. Chem. Phys. 1997, 107, 8221-8228.

186. Gillen, R.; Clark, S. J.; Robertson, J., Nature of the Electronic Band Gap in Lanthanide Oxides. Phys. Rev., B 2013, 87, 125116.

187. Jacobsson, T. J.; Correa-Baena, J.-P.; Pazoki, M.; Saliba, M.; Schenk, K.; Grätzel, M.; Hagfeldt, A., Exploration of the Compositional Space for Mixed Lead Halogen Perovskites for High Efficiency Solar Cells. Energy Environ. Sci. 2016, 9, 1706-1724.

188. Sutter-Fella, C. M.; Li, Y.; Amani, M.; Ager III, J. W.; Toma, F. M.; Yablonovitch, E.; Sharp, I. D.; Javey, A., High Photoluminescence Quantum Yield in Band Gap Tunable Bromide Containing Mixed Halide Perovskites. Nano Lett. 2015, 16, 800806.

189. Hu, M.; Bi, C.; Yuan, Y.; Xiao, Z.; Dong, Q.; Shao, Y.; Huang, J., Distinct Exciton Dissociation Behavior of Organolead Trihalide Perovskite and Excitonic Semiconductors Studied in the Same System. Small 2015, 11, 2164-2169.

190. Jeon, N. J.; Na, H.; Jung, E. H.; Yang, T.-Y.; Lee, Y. G.; Kim, G.; Shin, H.-W.; Seok, S. I.; Lee, J.; Seo, J., A Fluorene-Terminated Hole-Transporting Material for Highly Efficient and Stable Perovskite Solar Cells. Nat. Energy 2018, 3, 682. 
191. Green, M. A.; Hishikawa, Y.; Warta, W.; Dunlop, E. D.; Levi, D. H.; Hohl-Ebinger, J.; Ho-Baillie, A. W., Solar Cell Efficiency Tables (Version 50). Prog. Photovoltaics: Resear. Appl. 2017, 25, 668-676.

192. Mahmud, M. A.; Elumalai, N. K.; Upama, M. B.; Wang, D.; Wright, M.; Chan, K. H.; Xu, C.; Haque, F.; Uddin, A., Single vs Mixed Organic Cation for Low Temperature Processed Perovskite Solar Cells. Electrochim. Acta 2016, 222, 15101521.

193. Mohite, A.; Lin, J.-T.; Sumanasekera, G.; Alphenaar, B. W., Field-Enhanced Photocurrent Spectroscopy of Excitonic States in Single-Wall Carbon Nanotubes. Nano lett. 2006, 6, 1369-1373.

194. Mohite, A. D.; Gopinath, P.; Shah, H. M.; Alphenaar, B. W., Exciton Dissociation and Stark Effect in the Carbon Nanotube Photocurrent Spectrum. Nano lett. 2008, 8, $142-146$.

195. Mohite, A. D.; Santos, T. S.; Moodera, J. S.; Alphenaar, B. W., Observation of the Triplet Exciton in EuS-Coated Single-Walled Nanotubes. Nature Nanotech. 2009, 4 , 425-429.

196. Bansal, T.; Mohite, A. D.; Shah, H. M.; Galande, C.; Srivastava, A.; Jasinski, J. B.; Ajayan, P. M.; Alphenaar, B. W., New Insights into the Density of States of Graphene Oxide Using Capacitive Photocurrent Spectroscopy. Carbon 2012, 50, 808-814.

197. Tavakoli, M. M.; Yadav, P.; Prochowicz, D.; Sponseller, M.; Osherov, A.; Bulović, V.; Kong, J., Controllable Perovskite Crystallization via Antisolvent Technique Using Chloride Additives for Highly Efficient Planar Perovskite Solar Cells. Adv. Energy Mater. 2019, 9, 1803587.

198. Huang, Y.; Wu, S.; Chen, R.; Fang, S.; Zhang, S.; Wang, G.; Chen, W., Efficient Methylamine-Containing Antisolvent Strategy to Fabricate High-Efficiency and Stable FA0. 85Cs0. 15Pb (Br0. 15I2. 85) Perovskite Solar Cells. ACS Appl. Mater. Interfaces 2019, 11, 18415-18422.

199. Gu, C.; Lee, J.-S., Flexible Hybrid Organic-Inorganic Perovskite Memory. ACS Nano 2016, 10, 5413-5418. 
200. Meloni, S.; Moehl, T.; Tress, W.; Franckevičius, M.; Saliba, M.; Lee, Y. H.; Gao, P.; Nazeeruddin, M. K.; Zakeeruddin, S. M.; Rothlisberger, U., Ionic PolarizationInduced Current-Voltage Hysteresis in $\mathrm{CH}_{3} \mathrm{NH}_{3} \mathrm{PbX}_{3}$ Perovskite Solar Cells. Nat. Commun. 2016, 7, 10334.

201. Hu, H.; Salim, T.; Chen, B.; Lam, Y. M., Molecularly Engineered Organic-Inorganic Hybrid Perovskite with Multiple Quantum Well Structure for Multicolored LightEmitting Diodes. Sci. Rep. 2016, 6, 1-8.

202. Yakunin, S.; Dirin, D. N.; Shynkarenko, Y.; Morad, V.; Cherniukh, I.; Nazarenko, O.; Kreil, D.; Nauser, T.; Kovalenko, M. V., Detection of Gamma Photons Using Solution-Grown Single Crystals of Hybrid Lead Halide Perovskites. Nat. Photonics 2016, 10, 585.

203. Goldschmidt, V. M., Die gesetze der krystallochemie. Naturwissenschaften 1926, 14 , 477-485.

204. Shi, D.; Adinolfi, V.; Comin, R.; Yuan, M.; Alarousu, E.; Buin, A.; Chen, Y.; Hoogland, S.; Rothenberger, A.; Katsiev, K., Low Trap-State Density and Long Carrier Diffusion in Organolead Trihalide Perovskite Single Crystals. Science 2015, $347,519-522$.

205. Chen, H.-W.; Sakai, N.; Ikegami, M.; Miyasaka, T., Emergence of Hysteresis and Transient Ferroelectric Response in Organo-Lead Halide Perovskite Solar Cells. $J$. Phys. Chem. Lett. 2015, 6, 164-169.

206. Calado, P.; Telford, A. M.; Bryant, D.; Li, X.; Nelson, J.; O'Regan, B. C.; Barnes, P. R., Evidence for Ion Migration in Hybrid Perovskite Solar Cells with Minimal Hysteresis. Nat. Commun. 2016, 7, 13831.

207. Xing, G.; Mathews, N.; Sun, S.; Lim, S. S.; Lam, Y. M.; Grätzel, M.; Mhaisalkar, S.; Sum, T. C., Long-Range Balanced Electron-and Hole-Transport Lengths in OrganicInorganic $\mathrm{CH}_{3} \mathrm{NH}_{3} \mathrm{PbI}_{3}$. Science 2013, 342, 344-347.

208. Stranks, S. D.; Eperon, G. E.; Grancini, G.; Menelaou, C.; Alcocer, M. J.; Leijtens, T.; Herz, L. M.; Petrozza, A.; Snaith, H. J., Electron-Hole Diffusion Lengths 
Exceeding 1 Micrometer in an Organometal Trihalide Perovskite Absorber. Science 2013, 342, 341-344.

209. Xiao, Z.; Yuan, Y.; Shao, Y.; Wang, Q.; Dong, Q.; Bi, C.; Sharma, P.; Gruverman, A.; Huang, J., Giant Switchable Photovoltaic Effect in Organometal Trihalide Perovskite Devices. Nat. Mater. 2015, 14, 193-198.

210. Burschka, J.; Pellet, N.; Moon, S.-J.; Humphry-Baker, R.; Gao, P.; Nazeeruddin, M. K.; Grätzel, M., Sequential Deposition as a Route to High-Performance PerovskiteSensitized Solar Cells. Nature 2013, 499, 316.

211. Hu, J.; Gottesman, R.; Gouda, L.; Kama, A.; Priel, M.; Tirosh, S.; Bisquert, J.; Zaban, A., Photovoltage Behavior in Perovskite Solar Cells Under Light-Soaking Showing Photoinduced Interfacial Changes. ACS Energy Lett. 2017, 2, 950-956.

212. Zhao, C.; Chen, B.; Qiao, X.; Luan, L.; Lu, K.; Hu, B., Revealing Underlying Processes Involved in Light Soaking Effects and Hysteresis Phenomena in Perovskite Solar Cells. Adv. Energy Mater. 2015, 5, 1500279.

213. Shao, S.; Abdu-Aguye, M.; Sherkar, T. S.; Fang, H. H.; Adjokatse, S.; Brink, G. t.; Kooi, B. J.; Koster, L. J. A.; Loi, M. A., The Effect of the Microstructure on TrapAssisted Recombination and Light Soaking Phenomenon in Hybrid Perovskite Solar Cells. Adv. Funct. Mater. 2016, 26, 8094-8102.

214. Byun, H. R.; Park, D. Y.; Oh, H. M.; Namkoong, G.; Jeong, M. S., Light Soaking Phenomena in Organic-Inorganic Mixed Halide Perovskite Single Crystals. ACS Photonics 2017, 4, 2813-2820.

215. Yoon, S. J.; Draguta, S.; Manser, J. S.; Sharia, O.; Schneider, W. F.; Kuno, M.; Kamat, P. V., Tracking Iodide and Bromide Ion Segregation in Mixed Halide Lead Perovskites during Photoirradiation. ACS Energy Lett. 2016, 1, 290-296.

216. Hoke, E. T.; Slotcavage, D. J.; Dohner, E. R.; Bowring, A. R.; Karunadasa, H. I.; McGehee, M. D., Reversible Photo-Induced Trap Formation in Mixed-Halide Hybrid Perovskites for Photovoltaics. Chem. Sci. 2015, 6, 613-617.

217. Hentz, O.; Zhao, Z.; Gradečak, S., Impacts of Ion Segregation on Local Optical Properties in Mixed Halide Perovskite Films. Nano lett. 2016, 16, 1485-1490. 
218. Han, Y.; Meyer, S.; Dkhissi, Y.; Weber, K.; Pringle, J. M.; Bach, U.; Spiccia, L.; Cheng, Y.-B., Degradation Observations of Encapsulated Planar $\mathrm{CH}_{3} \mathrm{NH}_{3} \mathrm{PbI}_{3}$ Perovskite Solar Cells at High Temperatures and Humidity. J. Mater. Chem. A 2015, 3, 8139-8147.

219. Wu, X.; Trinh, M. T.; Niesner, D.; Zhu, H.; Norman, Z.; Owen, J. S.; Yaffe, O.; Kudisch, B. J.; Zhu, X.-Y., Trap States in Lead Iodide Perovskites. J. Am. Chem. Soc. 2015, 137, 2089-2096.

220. Galisteo-López, J. F.; Anaya, M.; Calvo, M.; Míguez, H., Environmental Effects on the Photophysics of Organic-Inorganic Halide Perovskites. J. Phys. Chem. Lett. 2015, 6, 2200-2205.

221. Bryant, D.; Aristidou, N.; Pont, S.; Sanchez-Molina, I.; Chotchunangatchaval, T.; Wheeler, S.; Durrant, J. R.; Haque, S. A., Light and Oxygen Induced Degradation Limits the Operational Stability of Methylammonium Lead Triiodide Perovskite Solar Cells. Energy Environ. Sci. 2016, 9, 1655-1660.

222. Christopher, P.; Xin, H.; Linic, S., Visible-Light-Enhanced Catalytic Oxidation Reactions on Plasmonic Silver Nanostructures. Nat. Chem. 2011, 3, 467.

223. Gao, F.; Xuan, W.; Bermak, A.; Boussaid, F.; Tsui, C.-Y.; Luo, J., Dual Transduction on a Single Sensor for Gas Identification. Sensor Actuat. B-Chem. 2019, 278, 21-27.

224. Tsai, H.; Asadpour, R.; Blancon, J.-C.; Stoumpos, C. C.; Durand, O.; Strzalka, J. W.; Chen, B.; Verduzco, R.; Ajayan, P. M.; Tretiak, S., Light-Induced Lattice Expansion Leads to High-Efficiency Perovskite Solar Cells. Science 2018, 360, 67-70.

225. Dalton, L. R.; Harper, A. W.; Robinson, B. H., The Role of London Forces in Defining Noncentrosymmetric Order of High Dipole Moment-High Hyperpolarizability Chromophores in Electrically Poled Polymeric Thin Films. Proc. Natl. Acad. Sci. 1997, 94, 4842-4847.

226. Da Silva, J. L.; Stampfl, C., Trends in Adsorption of Noble Gases He, Ne, Ar, Kr, and Xe on $\operatorname{Pd}(111)(\sqrt{3} \times \sqrt{3}) \mathrm{R} 30^{\circ}$ : All-Electron Density-Functional Calculations. Phys. Rev. B 2008, 77, 045401. 
227. Snaith, H. J.; Abate, A.; Ball, J. M.; Eperon, G. E.; Leijtens, T.; Noel, N. K.; Stranks, S. D.; Wang, J. T.-W.; Wojciechowski, K.; Zhang, W., Anomalous Hysteresis in Perovskite Solar Cells. J. Phys. Chem. Lett. 2014, 5, 1511-1515.

228. Wetzelaer, G. J. A.; Scheepers, M.; Sempere, A. M.; Momblona, C.; Ávila, J.; Bolink, H. J., Trap-Assisted Non-Radiative Recombination in Organic-Inorganic Perovskite Solar Cells. Adv. Mater. 2015, 27, 1837-1841.

229. Christians, J. A.; Fung, R. C.; Kamat, P. V., An Inorganic Hole Conductor for Organo-Lead Halide Perovskite Solar Cells. Improved Hole Conductivity with Copper Iodide. J. Am. Chem. Soc. 2013, 136, 758-764.

230. Fabini, D., Quantifying the Potential for Lead Pollution from Halide Perovskite Photovoltaics. ACS Publications: 2015.

231. Hao, F.; Stoumpos, C. C.; Cao, D. H.; Chang, R. P.; Kanatzidis, M. G., Lead-Free Solid-State Organic-Inorganic Halide Perovskite Solar Cells. Nat. Photon. 2014, 8 , 489-494.

232. Noel, N. K.; Stranks, S. D.; Abate, A.; Wehrenfennig, C.; Guarnera, S.; Haghighirad, A.-A.; Sadhanala, A.; Eperon, G. E.; Pathak, S. K.; Johnston, M. B., Lead-Free Organic-Inorganic Tin Halide Perovskites for Photovoltaic Applications. Energy Environ. Sci. 2014, 7, 3061-3068.

233. Hao, F.; Stoumpos, C. C.; Guo, P.; Zhou, N.; Marks, T. J.; Chang, R. P.; Kanatzidis, M. G., Solvent-Mediated Crystallization of $\mathrm{CH}_{3} \mathrm{NH}_{3} \mathrm{SnI}_{3}$ Films for Heterojunction Depleted Perovskite Solar Cells. J. Am. Chem. Soc. 2015, 137, 11445-11452.

234. Chen, Y.; Sun, Y.; Peng, J.; Tang, J.; Zheng, K.; Liang, Z., 2D Ruddlesden-Popper Perovskites for Optoelectronics. Adv. Mater. 2018, 30, 1703487. 


\section{APPENDIX}

\section{A1. List of Abbreviations}

$\mathrm{AC}$

ACP

AFM

ASTM

BA

$\mathrm{CB}$

$\mathrm{CE}$

CIGS

CN-PPV

QDSC

$\mathrm{CV}$

CZTS

DC

DMF

DSSC

PSC

EA

EIS

EQE

ETL

ETM

FA

FE

$\mathrm{FF}$

FTO

GA

HOMO alternating current

AC photocurrent

atomic force microscopy

American Society of Testing and Materials

n-butylammonium

conduction band

counter electrode

copper indium gallium diselenide

cyano-polyphenylene vinylene

quantum dot solar cell

cyclic voltammetry

copper zinc tin sulfide

direct current

$\mathrm{N}, \mathrm{N}$-dimethylformamide

dye-sensitized solar cell

perovskite solar cell

electron affinity

electrochemical impedance spectroscopy

external quantum efficiency

electron transport layer

electron transport material

formamidinium

fermi energy

fill factor

fluorine-doped tin oxide

guanidinium

highest occupied molecular orbital 


$\begin{array}{ll}\text { HP } & \text { hybrid perovskite } \\ \text { HTL } & \text { hole transport layer } \\ \text { HTM } & \text { hole transport material } \\ \text { IPCE } & \text { incident photon-to-electron conversion efficiency } \\ \text { ITO } & \text { indium tin oxide } \\ \text { LPF } & \text { long pass filter } \\ \text { LUMO } & \text { lowest unoccupied molecular orbital } \\ \text { MA } & \text { methylammonium } \\ \text { MACl } & \text { methylammonium chloride } \\ \text { MHHHPSC } & \text { mixed halide hybrid perovskite solar cell } \\ \text { MHHP } & \text { mixed halide hybrid perovskite } \\ \text { NP } & \text { nanoparticle } \\ \text { NTD } & \text { nanostructured titanium dioxide } \\ \text { OCP } & \text { open circuit potential } \\ \text { OSC } & \text { organic solar cell } \\ \text { PA } & \text { photoanode } \\ \text { PC } & \text { photocathode } \\ \text { PCE } & \text { photoconversion efficiency } \\ \text { PEC } & \text { photoelectrochemical } \\ \text { PFM } & \text { piezoresponse force microscopy } \\ \text { PPV } & \text { polyphenylene vinylene } \\ \text { PSC } & \text { perovskite solar cell } \\ \text { QC } & \text { quartz crystal } \\ \text { QCM } & \text { quartz crystal coated with perovskite } \\ \text { RE } & \text { reference electrode } \\ \text { REO } & \text { rare-earth oxide } \\ \text { RF } & \text { resonant frequency } \\ \text { RHE } & \text { reversible hydrogen electrode } \\ \text { RMS } & \text { root mean square } \\ \text { rpm } & \text { revolutions per minute } \\ \text { SEM } & \text { scanning electron microscopy } \\ \text { TCO } & \text { transparent conducting oxide } \\ \text { UV } & \text { ultraviolet } \\ \text { VB } & \text { valence band } \\ \text { WE } & \end{array}$




\section{A2. List of Symbols}

$\eta$

v

$\overline{\mathrm{N}}_{\mathrm{L}}$

$\mathrm{E}_{\mathrm{CB}}$

$\mathrm{E}_{\mathrm{f}}$

$\mathrm{E}_{\mathrm{in}}$

$\mathrm{E}_{\mathrm{VB}}$

Jark

Jinj

injection

$\mathrm{J}_{\max }$

JSC

l

$\mathrm{n}_{\mathrm{C}}$

$\mathrm{N}_{\mathrm{i}}$

$\mathrm{P}_{0}$

$\mathrm{P}_{\text {in }}$

$\mathrm{P}_{\max }$

$\mathrm{P}_{\text {out }}$

$\mathrm{P}_{\mathrm{v}}$

$\mathrm{r}_{\mathrm{A}}$

$\mathrm{r}_{\mathrm{B}}$

$\mathrm{r}_{\mathrm{X}}$

$\mathrm{V}_{\mathrm{bi}}$

$\mathrm{V}_{\mathrm{fb}}$

$\mathrm{V}_{\text {max }}$

$\mathrm{V}_{\mathrm{OC}}$

$\mathrm{V}_{\mathrm{p}}$

$\mathrm{Z}^{\prime \prime}$

$\beta_{\mathrm{n}}$

$\varepsilon_{0}$

$\varepsilon_{\mathrm{r}} / \varepsilon$

$\tau_{0}$ efficiency

frequency of radiation/scan rate

number of grain boundaries intersections per unit length

energy of conduction band

fermi energy

induced electric field

energy of valence band

dark current density

photocurrent density arising due to the total charge

photocurrent density corresponding to $\mathrm{P}_{\max }$ of solar cell

short-circuit current density

lineal size of the grain

density of electrons in the conduction band

number of grain boundaries intersected

polarization of pristine device

power of incident light on solar cell

maximum power dissipated by a solar cell

power obtained from solar cell

polarization of device by applying external potential

radius of ion $\mathrm{A}$

radium of ion $B$

radius of ion $\mathrm{X}$

built-in potential

flat-band potential

potential corresponding to $\mathrm{P}_{\max }$ of solar cell

open circuit potential

peak potential

imaginary part of impedance

time constant per unit volume of electron capture

permittivity of free space

relative dielectric constant

trapping lifetime of free electron at equilibrium 


$\begin{array}{ll}\tau_{\mathrm{dtr}} & \text { time constant for electron detrapping } \\ \tau_{\mathrm{tr}} & \text { time constant of electron trapping } \\ \mathrm{C} & \text { space charge capacitance } \\ \mathrm{e} & \text { charge on electron } \\ \mathrm{f} & \text { frequency of applied ac signal } \\ \mathrm{h} & \text { Planck's constant } \\ \mathrm{k} & \text { Boltzmann's constant } \\ \mathrm{L} & \text { length of test line } \\ \mathrm{M} & \text { magnification } \\ \mathrm{N} & \text { space charge density } \\ \mathrm{P} & \text { polarization } \\ \mathrm{q} & \text { elementary charge } \\ \mathrm{T} & \text { absolute temperature } \\ \mathrm{t} & \text { Goldschmidt's tolerance factor }\end{array}$




\title{
A3. Copyright Permissions
}

\author{
Reference \# 136
}

Title: Effect of Rare-Earth Metal Oxide Nanoparticles on the Conductivity of Nanocrystalline Titanium Dioxide: An Electrical and Electrochemical Approach Author: Pom L. Kharel, Paul M. Cuillier, Kasun Fernando, Bruce W. Alphenaar and Francis P. Zamborini

Publication: The Journal of Physical Chemistry C

Publisher: American Chemical Society

Date: Jul 1, 2018

Copyright (C) 2018, American Chemical Society

\section{PERMISSION/LICENSE IS GRANTED FOR YOUR ORDER AT NO CHARGE}

This type of permission/license, instead of the standard Terms \& Conditions, is sent to you because no fee is being charged for your order. Please note the following:

- Permission is granted for your request in both print and electronic formats, and translations.

- If figures and/or tables were requested, they may be adapted or used in part.

- Appropriate credit for the requested material should be given as follows: "Reprinted (adapted) with permission from (COMPLETE REFERENCE CITATION). Copyright (YEAR) American Chemical Society." Insert appropriate information in place of the capitalized words.

- One-time permission is granted only for the use specified in your request. No additional uses are granted (such as derivative works or other editions). For any other uses, please submit a new request.

Reference \# 169 Reprint with the permission from

Kharel, P. L, Zamborini, F. P., Alphenaar, B. W. J. Electrochem. Soc. 2018, 165, H52. 


\title{
CURRICULUM VITAE
}

\author{
Pom L. Kharel \\ University of Louisville \\ Department of Chemistry \\ Phone (502)517-6169 \\ pom.kharel@louisville.edu
}

\section{PROFESSIONAL GOAL}

- Innovative researcher in optoelectronic devices, electrocatalysis for energy conversion and nanoparticles for electrochemical sensing

- Leading role in an academic career

\section{EDUCATION}

- University of Louisville. Louisville, KY

Ph.D., Chemistry

July 2020

MS, Physical, Inorganic and Analytical Chemistry

May 2019

- Tribhuvan University. Kathmandu, Nepal

MS, Physical Chemistry (2008)

BS, Physics, Chemistry and Mathematics (2002)

\section{PROFESSIONAL EXPERIENCE}

Graduate Research Assistant (01/2016 - 07/2019)

Department of Chemistry, University of Louisville

Louisville, KY

Dissertation: Photoelectrical and Photoelectrochemical Characterization of the

Materials Used in Dye-Sensitized and Perovskite Solar Cells

Advisors: Dr. Francis P. Zamborini and Dr. Bruce P. Alphenaar 
Visiting Scholar (June-August 2019)

Department of Chemical and Biomolecular Engineering, Rice University Houston, TX

Founder and Managing Director (2011-2014)

Nepal Model Research Center and Nepal Model College

Kathmandu, Nepal

Research Fellow (2004-2008)

Central Department of Chemistry, Tribhuvan University

Kathmandu, Nepal

Thesis: Corrosion Behavior of W-Ni-Cr alloys in Aggressive Environments Advisor: Dr. Jagadeesh Bhattarai

\section{PUBLICATIONS}

1. Kharel, P. L.; Zamborini, F. P.; Alphenaar, B. W. Enhancing the Photovoltaic Performance of Dye-Sensitized Solar Cells with Rare-Earth Metal Oxide Nanoparticles. J. Electrochem. Soc. 2018, 165, H52-H56.

2. Kharel, P. L.; Cuillier, P. M.; Fernando, K.; Zamborini, F. P.; Alphenaar, B. W. Effect of Rare-Earth Metal Oxide Nanoparticles on the Conductivity of Nanocrystalline Titanium Dioxide: An Electrical and Electrochemical Approach. J. Phys. Chem. C 2018, 122, 15090-15096.

3. Pom L. Kharel and Jagadeesh Bhattarai. The Corrosion Behavior of SputterDeposited W-Cr-(4-15) Ni Alloys in NaOH Solutions. J. Npl. Chem. Soc. 2009, 24, 3-11.

4. Pom L. Kharel and Jagadeesh Bhattarai. Effects of Chromium and Tungsten on the Corrosion Behavior of W-Cr-Ni alloys in $0.5 \mathrm{M} \mathrm{NaCl}$ Solution. J. Inst. Sci. Technol. 2009, 16, 141-151 
5. Pom L. Kharel, Santosh P. Sah and Jagadeesh Bhattarai. Roles of Alloying Elements on the Passivity of W-xCr-yNi Alloys in Aggressive Environments. Npl. J. Sci. 2013, 14, 73-80.

6. Pom L. Kharel, Kasun Fernando, Francis P. Zamborini, and Bruce W. Alphenaar. An AC Photocurrent Method for Characterization of the Electric Polarization of Hybrid Perovskites (submitted to Materials Characterization).

7. Kasun Fernando, Pom L. Kharel, Devesa Canicoba Noelia, Hsinhan Tsai , Jacky Even, Mercouri G. Kanatzidis, Jean-Christophe Blancon, Wanyi Nie, Bruce W. Alphenaar and Aditya D. Mohite. Origin of Intrinsic Unipolar Memristor Action on a Single-Grain of Halide Perovskite (submitted to Scientific Reports).

8. Pom L. Kharel, Kasun Fernando, Jack Wei, Siraj Sidhik, Yafei Wang, Aditya D. Mohite, Francis P. Zamborini, and Bruce W. Alphenaar. Enhancing the Electric Polarization of Hybrid Perovskite due to Light Soaking in Argon (under preparation).

\section{RESEARCH EXPERIENCE}

- Synthesis and purification of metal halide hybrid perovskites

- Fabrication of nano to microfilms using screen-printing, spin-coating and chemical vapor deposition

- Characterization of materials with UV/Vis spectroscopy, atomic force microscopy (AFM), scanning electron microscopy (SEM), X-ray diffraction (XRD), Fourier transform infrared spectroscopy (FTIR) and Raman Spectroscopy.

- Highly controlled measurement of built-in potential of hybrid perovskite (2D and 3D) films in vacuum and other background gases

- Optimizing the operating conditions of optoelectronic devices

- Making large crystals in solution and study their properties

- Fabrication of perovskite solar cells, dye-sensitized solar cells and perovskite-based thin film

- Electrochemical analysis techniques: linear sweep, cyclic voltammetry, anodic stripping voltammetry, chronoamperometry, and electrochemical impedance spectroscopy

- Synthesis and characterization of nanoparticles 
- Linking nanoparticles to substrates through molecular linkers to make electrodes for various applications

- BET area, porosity and roughness measurements

\section{RESEARCH PRESENTATIONS}

- Graduate Student Regional Research Conference, University of Louisville (February 2020)

- Gordon Research Conference, Ventura, CA (January 4-10, 2020)

- Graduate Student Regional Research Conference, University of Louisville (February 2019)

- Graduate Student Regional Research Conference, University of Louisville (March 2018)

- Gordon Research Conference, Ventura, CA (January 6-12, 2018)

- RE3 Workshop, Louisville, KY (May 2018)

- 232nd Electrochemical Society Meeting, National Harbor, MA (October 2016)

\section{KNOWLEDGE AND SKILLS}

- Designing the synthesis of molecules

- Data analysis

- Graphing: Igor pro

- Computer: MS office for the preparation of manuscript, formatting documents and PowerPoint presentations

- Language: English, Nepali, Hindi and Assamese

- Programs: Lab-view, $\mathrm{C} / \mathrm{C}++$ programs, Python

\section{TEACHING EXPERIENCE}

- Graduate Teaching Assistant/Research Assistant (08/2014 -06/2020) for 3 years

University of Louisville, Louisville, KY

I taught General Chemistry 207-210 under the instruction of senior Professors

- Chemistry Lecturer (01/01/2011-06/30/2014) 
Kathmandu Model College, Kathmandu, Nepal

I taught undergraduate Physical, Inorganic and Organic chemistry, and laboratories

- Chemistry Lecturer (01/01/2011-06/30/2014)

Nepal Model College, Kathmandu, Nepal

I taught undergraduate Physical chemistry courses and laboratories

- Chemistry Instructor (2011-2014)

NAME, Institute for Medical Education, Kathmandu, Nepal

I taught MBBS preparatory courses from Chemistry.

- Science Teacher (2004-2009)

Nightingale International Higher Secondary School, Nepal

- Science Teacher (1998-2002)

New Light English Boarding High School, Butwal, Nepal

AWARDS

- GNAS Travel Award, University of Louisville (2018)

- Department Research Assistantship, University of Louisville (2016-2017)

- Academic Excellence Award, Kathmandu Model College, Nepal (2011, 2013, 2014)

- Best Teacher Award, Nightingale International HSS, Nepal (2006, 2007, 2008)

- Best Science Teacher, Ministry of Education, Nepal (2008)

LEADERSHIP

- Secretary, Nepal-America Society of Kentucky (2014-2019)

- Vice-President, Nepalese Students Association, University of Louisville (20172018)

- Head of Department of Chemistry, Kathmandu Model HSS, Nepal (2013-2014)

- Head of Department of Science, Nightingale International HSS, Nepal (20062009)

- Founder and Managing Director, Nepal Model Higher Secondary School, Nepal (2011-2014)

- Organizer of Science Fairs and Science Exhibition Competitions, Nepal. 